\title{
Elastizität von vernetzten semiflexiblen Biopolymersystemen
}

\author{
Dissertation \\ zur Erlangung des \\ mathematisch-naturwissenschaftlichen Doktorgrades \\ "Doctor rerum naturalium" \\ der Georg-August-Universität Göttingen \\ im Promotionsprogramm ProPhys \\ der Georg-August University School of Science (GAUSS)
}

vorgelegt von

Daniel Wilkin

aus Speyer

Göttingen, 2015 


\section{$\underline{\text { Betreuungsausschuss }}$}

Prof. Dr. Annette Zippelius,

Institut für Theoretische Physik,

Georg-August-Universität Göttingen

Prof. Dr. Reiner Kree,

Institut für Theoretische Physik,

Georg-August-Universität Göttingen

\section{Mitglieder der Prüfungskommission}

Referentin: Prof. Dr. Annette Zippelius, Institut für Theoretische Physik,

Georg-August-Universität Göttingen

Korreferent: Prof. Dr. Reiner Kree, Institut für Theoretische Physik, Georg-August-Universität Göttingen

Weitere Mitglieder der Prüfungskommission

Prof. Dr. Marcus Müller,

Institut für Theoretische Physik,

Georg-August-Universität Göttingen

Prof. Dr. Sarah Köster,

Institut für Röntgenphysik,

Georg-August-Universität Göttingen

Prof. Dr. Tim Salditt, Institut für Röntgenphysik,

Georg-August-Universität Göttingen

Dr. Claus Heussinger,

Institut für Theoretische Physik,

Georg-August-Universität Göttingen

Tag der mündlichen Prüfung: 09. Juni 2015 


\section{Inhaltsverzeichnis}

$\begin{array}{ll}\text { 1. Einleitung } & 1\end{array}$

2. Monte-Carlo-Simulationen von gegrafteten Weakly Bending Wormlike Chains 3

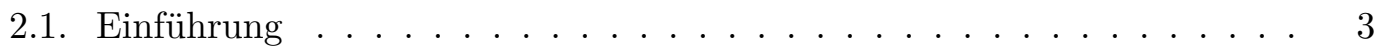

2.2. Anwendungsbereich und Ziel der Simulationen $\ldots . . . . . . . .77$

2.3. Modellierung der Biopolymere mit Hilfe der Coarse Graining-Methode . 7

2.4. Das Wormlike Chain-Modell . . . . . . . . . . . . . . . . . . 11

2.5. Ein Coarse Graining-Modell einer Polymerbürste . . . . . . . . . . . . . 13

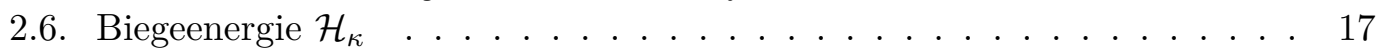

2.7. Crosslinkenergie $\mathcal{H}_{\mathrm{xlink}} \ldots \ldots \ldots \ldots \ldots \ldots \ldots$

2.8. Parameter-Einstellungen . . . . . . . . . . . . . . . . . . . . . . 19

2.9. Ordnungsmaße für die Grafting-Konfiguration . . . . . . . . . . . . . . 22

2.10. Allgemeine Informationen zur Monte-Carlo-Simulationsmethode. . . . . 25

2.11. Anwendung auf das Polymer Brush-Modell . . . . . . . . . . . . . . 30

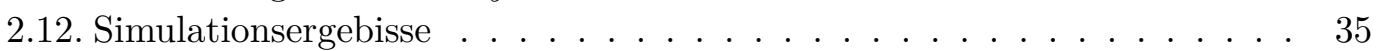

2.13. Einschränkungen der Systemgröße _. . . . . . . . . . . . . . . 35

2.14. Der statische Strukturfaktor $S(s, q) \ldots \ldots \ldots$

2.15. Domänengröße $l^{*} \ldots \ldots \ldots \ldots \ldots$. . . . . . . . . . . . . 41

2.16. Allgemeine Bemerkungen zur Bündelstruktur . . . . . . . . . . . . . . 42

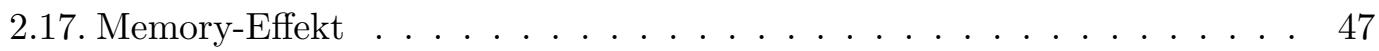

2.18. Mittlere Biege- und Gesamtenergie . . . . . . . . . . . . . . . . . . . . 49

2.19. Richtungsabhängige Korrelation der Segmente. . . . . . . . . . . . . . . 51

2.20. Transversale Fluktuationen . . . . . . . . . . . . . . . . . 54

2.21. Winkelverteilungen der Bond-Vektoren . . . . . . . . . . . . . . . 56

2.22. Segmentabhängige Crosslink-Dichte $\rho(s) \ldots \ldots \ldots$. . . . . . . 57

2.23. Mittlere Crosslink-Länge $l_{\mathrm{xl}} \ldots \ldots \ldots \ldots \ldots$. . . . . . . . . . 59

2.24. Zusammenfassung und Ausblick . . . . . . . . . . . . . . . . . . 61

3. Spannungs-Dehnungs-Kurve von vernetzten Biopolymeren 63

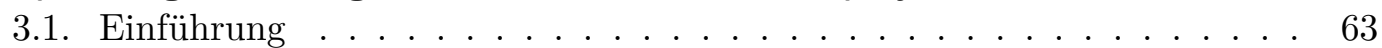

3.2. Beschreibung des Modellsystems . . . . . . . . . . . . . . . 65

3.3. Force-Extension mit einem Crosslink $(N=1)$. . . . . . . . . . . . . . . 69

3.4. Berechnung der Force-Extension für mehrere Crosslinks über die $\mathrm{Zu}-$

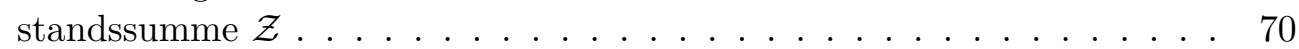

3.5. Berechnung der Spur von $\log \left(\mathbb{1}+C^{-1} U U^{T}\right)$ : . . . . . . . . . . . . 71

3.6. Ergebnis der Force-Extension mit Hilfe von $\mathcal{Z}_{\text {rel }}$. . . . . . . . . . . . . . 74

3.7. Berechnung von $G^{-1}$ mit Hilfe des Theorems von Cayley-Hamilton . . . 78

3.7.1. Rechenschritte . . . . . . . . . . . . . . 79 
3.8. Berechnung der Force-Extension mit Hilfe einer Reihenentwicklung . . . 84

3.9. Zusammenfassung und Ausblick . . . . . . . . . . . . . . . 86

\begin{tabular}{|rr}
\hline A. Anhang zum Simulationsteil & $\mathbf{8 7}$
\end{tabular}

A.1. Akzeptanzraten . . . . . . . . . . . . . . . . . . . . 8 87

A.2. Bend- und Crosslink-Moves . . . . . . . . . . . . . . . . . . . . 87

A.3. Bend-Move: Analytische Abschätzung der Akzeptanzrate . . . . . . . . 90

A.4. Diskrete Krümmung $c(s)$. . . . . . . . . . . . . . . . . . . 92

A.5. Richtungskorrelation $C_{t}(s)$ mit Excluded Volume-Wechselwirkung . . . . 93

A.6. Winkelverteilung unter dem Einfluss von Crosslinks. . . . . . . . . . . . 94

A.7. Alternatives Maß zur Messung des Quenched Disorder-Effektes . . . . . 95

\begin{tabular}{ll}
\hline B. Anhang zum analytischen Teil & 97
\end{tabular}

B.1. Der Erwartungswert von $J_{l}^{2}$ : . . . . . . . . . . . . . . . . . . 97

B.2. Erläuterungen zu $(N=1)$ Crosslinks . . . . . . . . . . . . . . . . . 98

B.3. Berechnung des Matrixelements $\tilde{p}_{m n} \ldots \ldots$. . . . . . . . . . . . 98

B.4. Angewandte Eigenschaften des Kronecker-Produktes . . . . . . . . . . . 99

B.5. Erläuterungen zu verwendeten Reihen . . . . . . . . . . . . . . . . . 100 


\section{Einleitung}

In biologischen Systemen spielen Makromoleküle eine wesentliche Rolle und beeinflussen den Verlauf von biologischen Prozessen maßgeblich. Eine charakteristische Eigenschaft von vielen dieser Biopolymere ist ihre Semiflexibilität [6, 35]. Das bedeutet, die Polymere sind durch eine im Vergleich zur flexiblen Gaußschen Kette höheren Biegesteifigkeit gekennzeichnet 9 .

Die Tatsache, dass semiflexible Polymere in biologischen Systemen wie zum Beispiel den eukaryotischen Zellen [31] in einer Vielzahl an Formen und Anordnungen erscheinen können [3, 4, 6], macht zahlreiche biologische Vorgänge erst möglich. Zu deren Verständnis wurden im Laufe der letzten Jahrzehnte viele experimentelle Untersuchungen In Vivo und In Vitro durchgeführt [26, 62,70,71] und außerdem theoretische Modelle entwickelt, die im Einklang mit den experimentellen Ergebnissen stehen [6, 35, 87.

In dieser aus zwei Teilen bestehenden Arbeit werden Modellsysteme studiert, die in vereinfachter Form speziellen, real existierenden biologischen Systemen nachempfunden sind.

- Im ersten Teil wird mit Hilfe numerischer Methoden 17, 91,111] eine Ansammlung von semiflexiblen Polymeren diskutiert, die senkrecht auf einer ebenen Substratoberfläche aufgepfropft (gegraftet) sind und über eine feste Anzahl an Crosslinks (auch als Crosslinkzahl bezeichnet) miteinander gekoppelt sind. Als Grundlage der theoretischen Beschreibung dient das Wormlike Chain (WLC)-Modell 72 100 in der Weakly Bending-Näherung 13, 87]. Es wird die Entstehung von Bündelstrukturen präsentiert, die beim Vorhandensein einer positiven Crosslink-Dichte und einer positiven Crosslink-Stärke auftreten. Des Weiteren kann festgestellt werden, dass eine persistente Bündelstruktur entsteht, falls die Grafting-Anordnung der Polymere unregelmäßig ist.

- Im zweiten Teil wird mit analytischen Methoden ein Modellsystem studiert, das aus zwei semiflexiblen Polymeren besteht, die durch eine beliebige Anzahl an $N$ irreversiblen und äquidistant angeordneten Crosslinks miteinander in Wechselwirkung stehen 13, 51, 87. Analog zum ersten Teil werden die beiden Polymerketten ebenfalls mit Hilfe des Wormlike Chain-Modells in der Weakly BendingNäherung beschrieben. Das Ziel bei dieser mathematischen Betrachtung besteht in der Berechnung der Spannungs-Dehnungs-Kurve (Force-Extension-Relation). Es kann gezeigt werden, dass diese auf analytischem Wege mit Hilfe verschiedener Lösungsansätze exakt lösbar ist. Außerdem kann abgeleitet werden, dass erwartungsgemäß die Steifigkeit des Systems sowohl mit zunehmender Crosslink-Stärke als auch mit steigender Crosslinkzahl nichtlinear erhöht wird. 



\section{Monte-Carlo-Simulationen von gegrafteten Weakly Bending Wormlike Chains}

\subsection{Einführung}

In diesem Teil präsentieren wir Monte-Carlo (MC)-Simulationen [17, 34, 91, 111] eines Systems, das aus parallel ausgerichteten und senkrecht zur ebenen Oberfläche aufgepfropften Polymeren besteht. Als Ausgangspunkt der hier präsentierten numerischen Analyse dient ein von Richard L. C. Vink entwickeltes Simulationsprogramm 111, 112 .

Die Polymere werden als Wormlike Chains in der Weakly Bending-Näherung beschrieben und benachbarte Ketten können reversibel mit Hilfe von Crosslinks miteinander verknüpft werden. Dabei werden die Crosslinks durch harmonische Federn modelliert, die eine attraktive Wechselwirkung zwischen den Polymerketten verursachen. Bei Erhöhung der Crosslinkzahl zeigen die Simulationen einen Übergang von einem homogenen Zustand zu einem Zustand, bei dem sich die Polymere in Form von Bündeln anordnen. Sind die Grafting-Positionen der Polymere auf dem Substrat zufällig verteilt, so erscheinen die Bündelstrukturen räumlich eingefroren, das heißt, die einmal angenommene Struktur wird nicht mehr aufgelöst. Sind die Polymere dagegen auf der Oberfläche äquidistant aufgepfropft, so sind die Bündel beweglich, das heißt, sie können sich jederorts bilden und wieder auflösen.

Biologische Zellen sind die fundamentalen lebenden Einheiten eines Organismus. Es sind Gebilde von enormer Komplexität, die über spezielle mechanische und dynamische Eigenschaften verfügen. Dies hängt mit der Fähigkeit ihrer Grundbausteine zu Selbstorganisation und Selbstaufbau zusammen [4, 31, 67. Diese Bestandteile besitzen die Eigenschaft, sich auf vielfältigste Weise in hochgeordneten Strukturen anordnen zu können, wodurch die viskoelastischen Eigenschaften der Zelle sowie ihre Form und Beweglichkeit maßgeblich beeinflusst werden [4,67,68.

So sind bei einer Zelle die Fähigkeit einer Umstrukturierung sowie die mechanische Stabilität von herausragender Bedeutung im Hinblick auf die Zellmorphologie sowie auf viele sich in der Zelle abspielenden Phänomene $35,57,68$. Wichtige Beispiele sind neben der Erhaltung der mechanischen Stabilität viele lebensnotwendige biologische Prozesse hinsichtlich des Wachstums und der Entwicklung eines Organismus, zum Beispiel 
intrazellulärer Transport, Zellexpansion, Zellteilung und Wundheilung [31,57, 68, 95].

Diese besonderen Eigenschaften einer lebenden Zelle sind weitestgehend auf das intrazelluläre Biopolymer-Gerüst, das Zytoskelett, zurückzuführen. Das Zytoskelett ist ein komplexes, filamentöses Netzwerk, das im Zytoplasma eukaryotischer Zellen enthalten ist. Die wichtigsten Bausteine dieses intrazellulären Polymer-Gerüsts sind drei Gruppen von Biopolymer-Proteinen, und zwar Actinfilamente (F-Actin), Intermediärfilamente wie zum Beispiel die Keratine und Mikrotubuli [4, 21, 67, 92 .

Im Bereich der Biophysik stellt das Zytoskelett seit vielen Jahren einen wichtigen Forschungsschwerpunkt dar [3, 4, 6, 31, 67, 80, 81. Um physikalische Sachverhalte im Zellinneren nachvollziehen zu können, das heißt, um Gesetzmäßigkeiten und Phänomene innerhalb eines komplexen Systems wie der Zelle erklären zu können, sind bestimmte Lösungsansätze notwendig. So konnten in der Vergangenheit mit gewissen Denkansätzen erfolgreich grundlegende Konzepte des Aufbaus und des Verhaltens sowie der inneren Organisation des Zytoskeletts aufgezeigt werden [57.

Nach wie vor tragen neben experimentellen Verfahren auch theoretische Ansätze zum Verständnis und der Funktionsweise biologischer Systeme bei [6, 13, 35, 38, 56, 64, 72, 74, 75, 87, 100. Anders gesagt ist ein Zusammenspiel zwischen theoretischen Modellbetrachtungen und experimentellen Untersuchungen unabdingbar, um ein umfassendes Verständnis der Mechanismen zu erlangen, die dem Verhalten von biologischen Systemen zugrunde liegen.

Die das Zytoskelett aufbauenden Filamente gehören wie DNS-Moleküle zur Klasse der semiflexiblen Polymere [6, 35, 66, 68, 86]. Sie verfügen über eine bestimmte Biegesteifigkeit (Bending Stiffness) $\kappa$, mit der stets eine endliche Persistenzlänge $L_{p}$ als charakteristisches Steifigkeitsmaß verbunden ist 35,64 .

Semiflexible Polymere weisen Persistenzlängen auf, die in der Größenordnung ihrer Konturlänge liegen [35, 93]. Auf über $L_{p}$ hinausgehenden großen Längenskalen stellt man bei ihnen ein exponentiell abklingendes Verhalten der Orientierungskorrelation seiner Polymersegmente fest, das heißt das Polymer erscheint in diesem Fall wie ein flexibles Polymer 65 75. Auf im Vergleich zur Persistenzlänge kleinen Längenskalen allerdings hat die Biegeenergie einen großen Einfluss auf das Verhalten des Polymers 65, auf sehr kleinen Längenskalen verhalten sich die Kettenglieder stabartig (rodlike) 56 . Aufgrund der Eigenschaft der Semiflexibilität der Polymere muss daher zum Verständnis ihrer statistischen Mechanik außer dem entropischen Beitrag die Biegeenergie der Filamente berücksichtigt werden 35 .

Die Biegesteifigkeit der Biopolymere kann stark variieren und hängt von der jeweiligen zu erfüllenden Aufgabe ab, sie bestimmt viele wichtige Eigenschaften des Polymers wie zum Beispiel seine Gestalt sowie die Größe der thermischen Fluktuationen [14. 68]. Dabei erfahren Filamente durch die Einbettung (Confinement) in ein dichtes Zytoplasma oder in Mikrokanäle starke geometrische Zwangsbedingungen, die sich auf die thermischen Fluktuationen und die Tangens-Korrelationsfunktion auswirken 70 , 71.

Actinfilamente und Mikrotubuli sind mit deutlich voneinander abweichenden Persistenzlängen ausgestattet. Mit Hilfe fluoreszenzmikroskopischer Methoden konnte zum 
Beispiel bei isolierten Actinfilamenten eine Persistenzlänge von $L_{p} \approx 17 \mu m$ nachgewiesen werden [66, 93]. Mikrotubuli sind dagegen deutlich steifer und besitzen dementsprechend eine höhere Persistenzlänge von $L_{p} \approx 5,2 \mathrm{~mm}$, wie aus der Messung der Steifigkeit durch die Analyse thermischer Fluktuationen festgestellt werden konnte [41. Die im Zytoskelett vorkommenden Proteine haben eine Konturlänge in der Größenordnung von einigen Mikrometern. In In Vitro-Experimenten konnte zum Beispiel gezeigt werden, dass das Protein Actin zu Actinfilamenten mit einer Länge von mindestens $L=20 \mu m$ polymerisieren kann 41 .
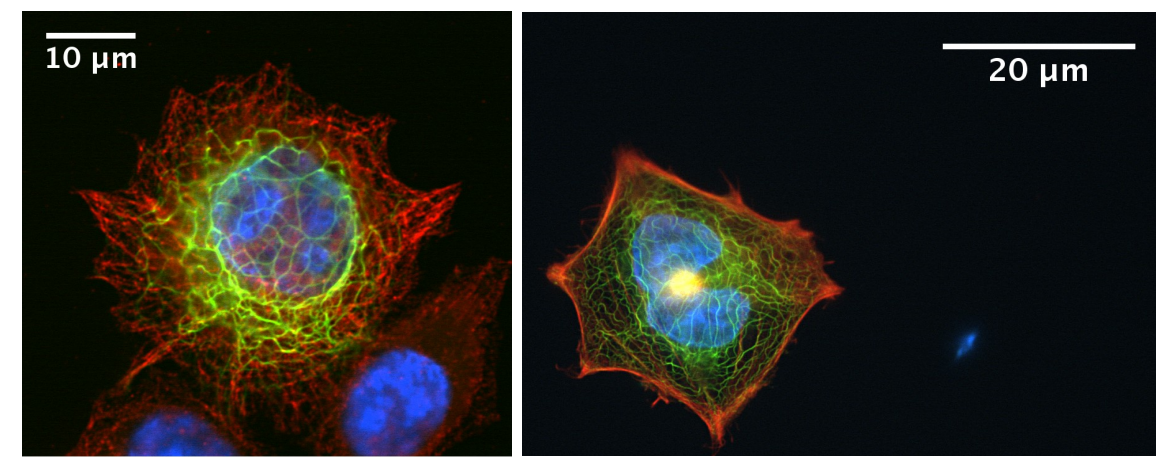

Abbildung 2.1.: Fluoreszenzmikroskopische Aufnahmen des Zytoskeletts. Der Zellkern mit DNS erscheint beiderseits in Blau, Keratin beiderseits in Grün. Durch rote Farbe werden in der Aufnahme links Mikrotubuli, in der Aufnahme rechts dagegen Actinfilamente sichtbar gemacht. Aufnahmen von B. Weinhausen 115

Zwei fluoreszenzmikroskopische Aufnahmen des eukaryotischen Zytoskeletts zeigt Abbildung (2.1) [115]. Mit fluoreszierenden Stoffen werden verschiedene Bestandteile des Zytoskeletts in unterschiedlichen Farben hervorgehoben. So erscheinen die Zellkerne in Blau und das Keratin-Netzwerk in Grün. Mit der Farbe Rot sind auf dem linken Bild die Mikrotubuli, auf der Aufnahme rechts dagegen die Actinfilamente hervorgehoben.

Die Mannigfaltigkeit an Formen, die Actinfilamente annehmen können, ist nur aufgrund von actin-bindenden Proteinen (ABP) wie zum Beispiel Fimbrin oder Fascin möglich [4]. In der Natur gibt es eine enorme Vielfalt an actin-bindenden Proteinen, die einen großen Einfluss auf die Mikrostruktur sowie die mechanischen und viskoelastischen Eigenschaften des Zytoskeletts haben 3, 4. In In Vitro-Experimenten wird der Einfluss zahlreicher biochemischer Eigenschaften von reversiblen, actin-bindenden Proteinen mit Hinblick auf die physikalischen Eigenschaften der Actin-Netzwerke überprüft [61, 82, 118]. Dabei kommt insbesondere der Reversibilität der Filament-Bindungen hinsichtlich der rheologischen Eigenschaften wie Fluidisierung einerseits und Verstärkung andererseits eine Schlüsselrolle zu [112.

Bei Untersuchungen des elastischen Verhaltens eines In Vitro-Netzwerkes aus gebündeltem und mit Crosslinks vernetztem F-Actin wurde gezeigt, wie sich deren Steifigkeit durch Variation der Actin- oder ABP-Konzentration verändern lässt 37.

Die actin-bindenden Proteine verfügen über zwei adhäsive Enden, mit denen ebenso viele Filamente miteinander verknüpft werden können [67]. Sie bewirken dadurch eine 
attraktive Wechselwirkung zwischen diesen beiden Proteinsträngen und werden gemäß ihrer Funktion als Crosslinker bezeichnet. Die Verbindungen zwischen Crosslinks und Actinfilamenten, auf denen die vielfältigen Mikrostrukturen beruhen, sind in der Regel reversibel. Das heißt, eingegangene Crosslink-Verbindungen zwischen Filamenten können mit Hilfe thermischer Energie wieder aufgehoben werden 67, 82.

Neben der Verknüpfung von Filamenten zu Netzwerken können die Crosslinker-Proteine Bündelstrukturen ermöglichen, das heißt, die Filamente sind axial und damit parallel zueinander ausgerichtet und relativ dicht gepackt [3, 4, 66, 67]. Actinbündel können in einer Vielzahl von komplexen Strukturen auftreten, sie dienen unter anderem der Stabilisierung von zellulären Einstülpungen oder Domänen der Plasma-Membran 3.4 . Durch die mit Hilfe von Crosslinks verursachte kooperative Wechselwirkung zwischen den Actinfilamenten und der damit verbundenen Entstehung einer Bündelstruktur geht ein Zustand höherer Ordnung einher.

An gegrafteten Polymersystemen wurde in der Vergangenheit innerhalb vieler physikalischer Bereiche geforscht, etwa in der Adhäsion oder Adsorption sowie der Gestaltung von Mustern auf Oberflächen oder dünnen Filmen [85, 89, 116, 119]. Zum Beispiel wurde bei gegrafteten, semiflexiblen Polymeren mit analytischen und numerischen Methoden die entropische Kraft untersucht, die bei Vorgängen innerhalb einer Zelle von Bedeutung ist 40 .

Ansammlungen von auf einer Oberfläche aufgepfropften semiflexiblen Polymeren werden aus Gründen der besseren Veranschaulichung oft als Polymerbürste (Polymer Brush) bezeichnet.

In Monte-Carlo-Simulationen wurden Selbstanordnungsprozesse bei supramolekularen Polymeren analysiert, die auf schwachen Interketten-Wechselwirkungen basieren [58]. Dabei konnte durch Senkung der Temperatur oder durch Erhöhung der Teilchendichte ein Phasenübergang erster Ordnung zur Bildung von soliden Polymerbündeln festgestellt werden.

Untersuchungen zum Auftreten von Bündelformationen wurden ferner in einer Kombination aus theoretischen Überlegungen und MC-Simulationen durchgeführt 66. Die theoretischen Betrachtungen beruhten auf parallel ausgerichteten semiflexiblen Polymeren, zwischen denen eine attraktive Wechselwirkung durch Crosslinker-Moleküle mit zwei adhäsiven Endgruppen vermittelt wird. Es konnte nachgewiesen werden, dass eine kritische Konzentration an Crosslinks benötigt wird, um einen diskontinuierlichen Phasenübergang von gleichförmig verteilten Filamenten zur Formation eines oder mehrerer Bündel zu ermöglichen.

Darüber hinaus wurden Monte-Carlo-Simulationen in Ergänzung zu analytischen Berechnungen eingesetzt, um die Response eines reversibel durch Crosslinks verknüpften Filamentbündels zu untersuchen, das einer äußeren Krafteinwirkung bzw. Biegedeformation ausgesetzt ist [112]. Es zeigte sich, dass eine zunehmende Krümmung des Bündels zu einer Reduzierung der Crosslink-Dichte führt, was auf einen kooperativen Unbinding Process hindeutet.

Im Allgemeinen gilt, dass Phasenübergänge zwischen gebündelten und ungebündelten Zuständen ihren Ursprung im Wechselspiel zwischen der mit transversalen Auslen- 
kungen verbundenen Entropie und der aus der attraktiven Wechselwirkung zwischen Filamenten herrührenden potentiellen Energie haben [8, 14, 65].

Reversibel durch Crosslinks verbundene, auf ein Substrat aufgepfropfte Polymerbürsten wurden mit Hilfe von Experimenten mit Rasterkraftmikroskopie auf mechanische Eigenschaften studiert 85. Im Fokus des Interesses stehen dabei auch potentielle technische Anwendungen von Polymerbürsten-Schichten in der biomedizinischen Ingenieurwissenschaft.

MC-Simulationen sind darüber hinaus anwendbar zur Analyse von Polymerketten, die auf einer ebenen Oberfläche aufgepfropft sind [77, 78]. Systeme von gegrafteten semiflexiblen Polymeren in einer verdünnten Lösung wurden studiert, sie offenbarten die Formierung verschiedener morphologischer Mizellen-Strukturen 94, 96]. Die Kettenkonformationen wurden dabei durch die Entropie der Kette sowie der Enthalpie der Kette-Lösungsmittel-Wechselwirkung bestimmt.

\subsection{Anwendungsbereich und Ziel der Simulationen}

Wir beschäftigen uns im Folgenden mit numerischen Untersuchungen, die sich auf reale Bausteine des Zytoskeletts beziehen. Im Rahmen eines eindimensionalen Modells wird mit Hilfe von Monte-Carlo-Simulationen das Verhalten von semiflexiblen, parallel ausgerichteten Polymerketten untersucht, die rechtwinklig auf einer Substratoberfläche aufgepfropft sind und über Crosslinks attraktive und kollektive Wechselwirkungen aufeinander ausüben. Das gesamte Modellsystem befindet sich in Kontakt mit einem Wärmereservoir der Temperatur $T$.

Dies geschieht mit Bezug zum realen Pendant der biologischen Zelle, indem ein vereinfachtes System, bestehend aus Actinfilamenten und actin-bindenden Crosslinker-Proteinen unter dem Einfluss thermischer Energie betrachtet wird.

Die Tatsache, dass die Polymerketten an einem Ende fest sind, reduziert ihre Freiheitsgrade bezüglich der möglichen Kettenkonformationen. Wir untersuchen also Phänomene von Makromolekülen in einer eingeschränkten Umgebung.

Das Ziel unserer Untersuchung besteht darin, festzustellen, unter welchen Umständen Bündelstrukturen entstehen und wie sich diese unter dem Einfluss thermischer Energie verhalten. Eine Grundannahme dabei ist, dass die Polymerketten als semiflexibel betrachtet werden können, das heißt, wir analysieren Ketten mit einer relativ hohen Biegesteifigkeit.

\subsection{Modellierung der Biopolymere mit Hilfe der Coarse Graining-Methode}

Wie im vorangegangenen Kapitel beschrieben, besteht seit vielen Jahren ein hohes Interesse am Verständnis von biophysikalischen Prozessen. Um das Verhalten von miteinander wechselwirkenden Filamenten in komplexen biologischen Systemen und den 
damit verbundenen kollektiven Phänomenen verstehen zu können, bedarf es neben experimentellen Untersuchungen der Modellierung von biologischen Systemen. Im Fokus des Interesses steht in unserem Fall ein System, das aus semiflexiblen Polymeren besteht, die über federartige Crosslinker-Moleküle miteinander in Wechselwirkung treten können. Trotz einer weitgehenden Vereinfachung der Polymerketten ist das Modell zur Untersuchung der thermischen Bewegungen und damit der entropischen Eigenschaften geeignet.

Realistische biologische Systeme auf einer atomistischen Grundlage analytisch zu behandeln, erweist sich aufgrund der hohen Teilchenzahl und der damit verbundenen hohen Zahl an Freiheitsgraden und der zu lösenden Bewegungsgleichungen als ein faktisch unmögliches Unterfangen. Auch für Computersimulationen ist es aussichtslos, bei einer genügend hohen Teilchenzahl sämtliche Bewegungsgleichungen zu lösen und alle mikroskopischen Details zu berücksichtigen. Eine mikroskopische Betrachtung eines Polymers würde daher aufgrund seiner vielen inneren Freiheitsgrade zu einer erheblichen Verkomplizierung der Analyse führen [50,101.

So sind im Hinblick auf anzuwendende Monte-Carlo-Simulationstechniken 17, 34, 91, 111. gewisse Vereinfachungen des zu studierenden Modells notwendig. Zum Einen, um eine Berechnung innerhalb einer angemessenen Zeit zu ermöglichen, zum Anderen werden Größen von Systemen mit Hilfe von Näherungen und Vereinfachungen überhaupt erst rechnerisch zugänglich.

Um Systeme mit einer hohen Anzahl an Freiheitsgraden untersuchen zu können, erweisen sich deshalb die Konzepte und Methoden der statistischen Mechanik 101 als ein nützliches Werkzeug. Zur Beschreibung unseres Modellsystems sind wir deshalb bestrebt, den Formalismus der statistischen Physik 50, 64, 101 anzuwenden, der im Allgemeinen zur Behandlung von Vielteilchensystemen wie Festkörper oder Biopolymere herangezogen wird. Das Konzept der statistischen Physik ist eine wichtige Basis zur theoretischen Behandlung von Biopolymeren. Es beruht auf der Betrachtung der Konformation und der konformativen Bewegung der Polymerketten auf großen räumlichen und zeitlichen Skalen und ermöglicht die Bestimmung der wichtigsten Eigenschaften von Biopolymeren [46].

Für die Kenntnis der universellen mesoskopischen Eigenschaften sind mikroskopische Details des Systems irrelevant, so dass mikroskopische Freiheitsgrade unberücksichtigt bleiben [64].

Dieses Konzept unterscheidet sich von der Polymerchemie, bei der die chemische bzw. atomistische Zusammensetzung eines Monomers berücksichtigt wird, was zu einer hohen Auflösung durch eine Betrachtung auf mikroskopischer Skala führt [99]. Während demnach chemische Eigenschaften stark von der jeweiligen atomistischen Zusammensetzung eines Polymers abhängen, sind allgemeine physikalische und mechanische Charakteristiken davon unabhängig [64. In unserem Fall spielt die chemische Zusammensetzung eines Monomers keine Rolle, das heißt, die Betrachtung erfolgt auf einer größeren, mittleren Längenskala. So kann man sagen, dass die statistische Polymerphysik von einer verallgemeinerten Beschreibung ausgeht, bei der eine Polymerkette aus einheitlichen, nicht näher spezifizierten Monomereinheiten zusammengesetzt ist 64, 108]. 
Auf Grundlage der Struktur bzw. der Konformation der Polymere können wichtige physikalische Eigenschaften auf einer mesoskopischen Skala hergeleitet werden [39,64]. Die konformativen Eigenschaften bestimmen das grundlegende Verhalten der Biopolymere und die bedeutendsten Phänomene, die auf der Konformation der Polymerketten basieren, laufen auf einer im Vergleich zur atomaren Größenordnung deutlich größeren Skala ab 46 .

Hinsichtlich der molekularen Beschreibung eines realen biologischen Systems im Rahmen der statistischen Physik wenden wir uns der Modellierung von Polymerketten zu. In unserem Fall wird das für ein Actinfilament stehende Biopolymer einer sehr starken Vereinfachung unterworfen. Actinfilamente sind aus vielen Monomereinheiten, dem globularen G-Actin, zusammmengesetzt und erscheinen in Form einer doppelsträngigen Helixstruktur [53. Wir reduzieren gemäß der Coarse Graining-Methode [5, 64] die Anzahl seiner Freiheitsgrade, indem sich wiederholende identische Molekülblöcke, die aus vielen Atomeinheiten zusammengesetzt sind, in Form eines einzelnen, punktförmigen Polymersegments zusammengefasst werden.
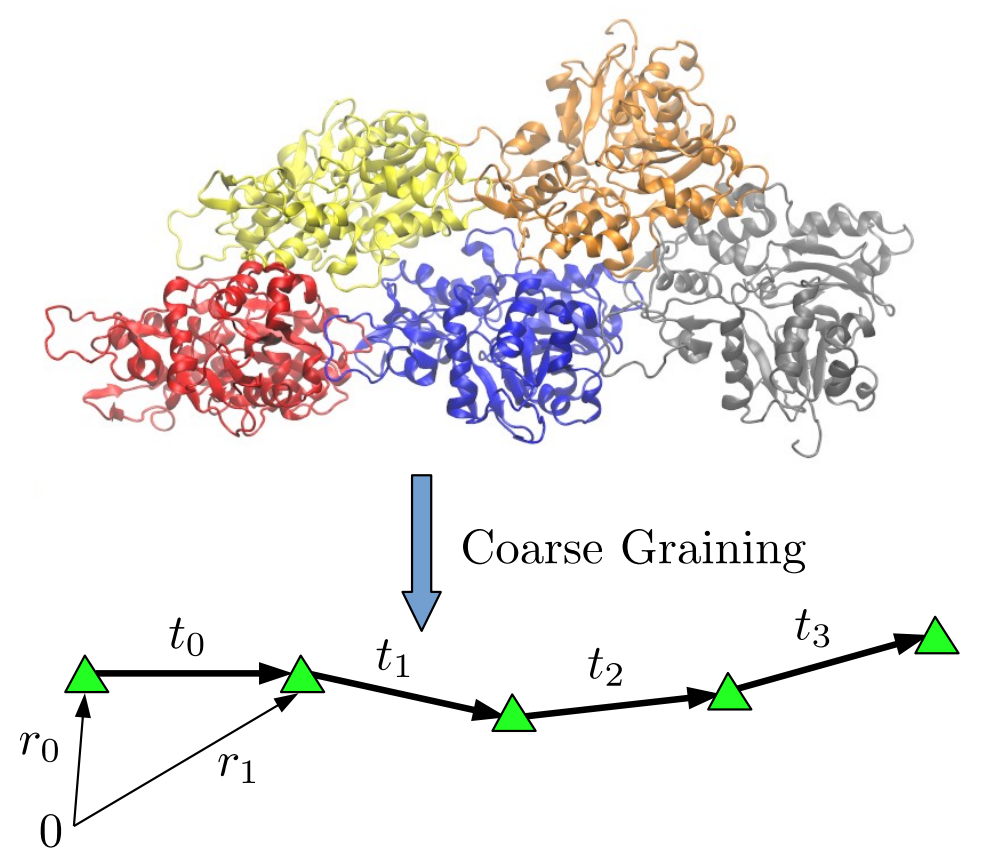

Abbildung 2.2.: Darstellung eines Ausschnitts von F-Actin mit Übergang zum Coarse Graining-Kettenmodell. Protein Data Bank (PDB)-Datei aus Publikation von E. Behrmann et al. 7], Bild gerendert mit VMD [59, modifiziert durch eigene Ergänzung.

Die Segmente oder auch Monomereinheiten stellen die polymerbildenden Grundbausteine im Rahmen der Coarse Graining-Vereinfachung dar. Darüber hinaus wird in der Modellierung der Ketten die Doppelhelixstruktur durch ein einfaches kettenförmiges Makromolekül vereinfacht. Die vollständige, molekulare Beschreibung der linearen Polymerkette beinhaltet die kettenförmige Verbindung der Monomereinheiten durch starre, stabartige Verbindungsglieder (Bonds) [46,64, wodurch eine wesentliche Vereinfa- 
chung des Modellsystems resultiert. Folglich werden, wie bereits erwähnt, molekülspezifische Details sowie die Sekundärstruktur der Doppelhelix des Makromoleküls [4,53, 84 ausgeblendet, so dass im Rahmen des verwendeten Modells eine allgemeine Betrachtung des Systems erfolgt, für das weder die genaue chemische Zusammensetzung noch die Sekundärstruktur des Kettenmoleküls erheblich ist.

Im Einklang mit dem Aufbau eines Actinfilaments aus identischen G-Actin-Moleküleinheiten [53] analysieren wir darüber hinaus ausschließlich Homopolymere 99, das heißt, alle Ketten zeichnen sich durch den gleichen Monomertyp aus. Im Modellsystem liegen außerdem ausschließlich Ketten gleicher Länge und gleicher Segment-Anzahl vor.

Wir beschreiben die Konformation eines Polymers, das aus $(L+1)$ Monomeren besteht, mit der Angabe der $(L+1)$ dazugehörigen Positionsvektoren 46,64

$$
\left\{\boldsymbol{r}_{0}, \boldsymbol{r}_{1}, \ldots, \boldsymbol{r}_{L}\right\}
$$

Dabei werden die Segmente vom Kettenanfang an durchnummeriert. Hieraus kann unmittelbar die Menge der L Bond-Vektoren

$$
\left\{\boldsymbol{t}_{0}, \boldsymbol{t}_{1}, \ldots, \boldsymbol{t}_{L-1}\right\} \quad \text { mit } \boldsymbol{t}_{s}=\boldsymbol{r}_{s+1}-\boldsymbol{r}_{s} ; s=0,1, \ldots,(L-1)
$$

die die Bindungen zwischen zwei benachbarten Segmenten derselben Kette symbolisieren, abgeleitet werden. Zur Erlangung einer genaueren Vorstellung von der Vereinfachung durch die Coarse Graining-Methode dient Abbildung (2.2). Dort ist schematisch der Übergang von einem komplexen, realitätsnahen Modell eines F-Actin-Abschnitts (vergleiche Modell von K. C. Holmes et al. [53]) zu einer einfachen, linearen Kette mit identischen Monomereinheiten gezeigt. Die sich im kurzen Ausschnitt der Grafik wiederholenden Untereinheiten des F-Actin-Stranges werden auf punktförmige Segmente (hier durch schwarz umrandete grüne Dreiecke symbolisiert) reduziert.

Gehen wir davon aus, dass die $(L+1)$ punktförmigen Segmente durch Bindungen konstanter Länge miteinander verbunden sind und folglich $\left|\boldsymbol{t}_{s}\right|=b \forall s$ gilt, so ergibt sich eine Gesamtlänge der Kette von $L_{c}=L \cdot b$.

Die thermischen Fluktuationen semiflexibler Polymere und Filamente werden durch das Verhältnis von Biegesteifigkeit $\kappa$ und thermischer Energie $k_{B} T$ reguliert, dies führt uns zum Konzept der Persistenzlänge [47.75]. Die Persistenzlänge $L_{p}$ stellt ein Maß zur Quantifizierung der Kettenflexibilität dar. Sie gibt die charakteristische Längenskala entlang des Polymers an, über die die Tangenteneinheitsvektoren der Polymerkette dekorrelieren und bestimmt somit die Orientierungskorrelation zwischen zwei Segmenten eines Makromoleküls [47, 50]. So erscheinen Filamente oder Polymere mit einer Konturlänge $L \ll L_{p}$ wie starre, dagegen bei $L \gg L_{p}$ wie flexible Makromoleküle.

Ist keine thermische Energie vorhanden, so sind thermische Fluktuationen vollständig unterdrückt und die Ketten sind exakt gerade ausgerichtet. Bei einer endlichen, von Null verschiedenen Temperatur dagegen führt das Polymer Fluktuationsbewegungen aus. 


\subsection{Das Wormlike Chain-Modell}

Zur statistischen Analyse flexibler Polymerketten im thermischen Gleichgewicht wird in der Regel die Freely Jointed Chain oder das Gaußsche Kettenmodell herangezogen 23 , 64]. Zur Beschreibung von semiflexiblen Polymerketten allerdings können diese Modelle nicht angewendet werden, stattdessen verwenden wir als theoretische Grundlage der Polymerketten das Wormlike Chain-Modell, das zuerst von O. Kratky und G. Porod formuliert wurde [72,100]. Es basiert darauf, dass die Biegung des Polymers mit der Aufwendung einer Biegeenergie verbunden ist, die proportional zur Biegesteifigkeit ist.

Die Polymerkette wird durch eine ebene und differenzierbare Raumkurve $\boldsymbol{r}(s)$ mit der Konturlänge $L_{c}$ und dem Parameter der Bogenlänge $s \in\left[0, L_{c}\right]$ beschrieben. Um die Kettensteifigkeit einer semiflexiblen Kette zu modellieren, gehen wir von einem linearen Coarse Graining-Kettenmodell aus. Die flexible, freie Rotation der einzelnen Bonds wird durch eine elastische Biegeenergie $\mathcal{W}_{\text {bend }}$ eingeschränkt. Dabei geht man von einem harmonischen Potential bezüglich des Winkels zweier benachbarter BondVektoren $\boldsymbol{t}_{s}$ und $\boldsymbol{t}_{s+1}$ aus 64]:

$$
\mathcal{W}_{\text {bend }}=\frac{\kappa_{0}}{2} \sum_{s=0}^{L-1}\left|\boldsymbol{t}_{s}-\boldsymbol{t}_{s+1}\right|^{2}=-\kappa_{0} \sum_{s=0}^{L-1} \boldsymbol{t}_{s} \cdot \boldsymbol{t}_{s+1}+\text { konst. }
$$

mit der unskalierten Biegesteifigkeit $\kappa_{0}$. Die elastische Biegeenergie im diskreten Fall 2.3 offenbart die Analogie zum eindimensionalen Heisenberg-Modell für Ferromagneten [101]. Wir gehen von diesem diskreten Fall in den Kontinuumslimes über und halten die Konturlänge konstant, das heißt, es gilt $N \rightarrow \infty, b \rightarrow 0$ und $\kappa_{0} \rightarrow \infty$ mit $\kappa_{0} / N=$ konstant. Mit dem Tangentenvektor $\boldsymbol{t}(s)$ und

$$
\left(\frac{\mathrm{d} \boldsymbol{t}(s)}{\mathrm{d} s}\right)=\lim _{b \rightarrow 0}\left(\frac{\boldsymbol{t}_{s+1}-\boldsymbol{t}_{s}}{b}\right)
$$

geht die Biegeenergie

$$
\mathcal{W}_{\text {bend }}=\lim _{b \rightarrow 0, N, \kappa_{0} \rightarrow \infty} \frac{\kappa_{0} b}{2} \sum_{s=0}^{L-1} b \cdot\left(\frac{\boldsymbol{t}_{s+1}-\boldsymbol{t}_{s}}{b}\right)^{2}
$$

mit $\sum_{s=0}^{L-1} b \rightarrow \int_{0}^{L_{c}} \mathrm{~d} s$ und der (skalierten) Biegesteifigkeit $\kappa=b \kappa_{0}$ in den kontiuierlichen Fall über [50]. Die statistischen Eigenschaften der Wormlike Chain werden somit durch den Hamiltonian der elastischen Biegeenergie

$$
\mathcal{H}[\boldsymbol{r}(s)]=\frac{\kappa}{2} \int_{0}^{L_{c}} \mathrm{~d} s\left(\frac{\mathrm{d} \boldsymbol{t}(s)}{\mathrm{d} s}\right)^{2}=\frac{\kappa}{2} \int_{0}^{L_{c}} \mathrm{~d} s\left(\frac{\mathrm{d}^{2} \boldsymbol{r}(s)}{\mathrm{d} s^{2}}\right)^{2}
$$

bestimmt 74,75$]$. Die elastische Biegeenergie ergibt sich demnach aus der Kettenkonformation, indem über die Quadrate der lokalen Krümmungen entlang der Bogenlänge $s$ integriert wird, unter Berücksichtigung des konstanten Faktors der Biegesteifigkeit $\kappa$. Die Biegesteifigkeit steht mit der Persistenzlänge $L_{p}$ über die Beziehung $L_{p}=2 \kappa /\left((D-1) k_{B} T\right)$ in Verbindung, wobei $D=2$ die Dimension des Raumes angibt [35]. Im zweidimensionalen Raum und mit $k_{B} \equiv 1$ vereinfacht sich die Relation zu 
$L_{p}=2 \kappa / T$. Ein wesentliches Charakteristikum der Wormlike Chain ist die lokale Undehnbarkeits-Zwangsbedingung (Local Inextensibility Constraint) $|\boldsymbol{t}(s)|=1$ 35, 64, 75, mit dem Tangenten-Einheitsvektor

$$
\boldsymbol{t}(s)=\frac{\mathrm{d} \boldsymbol{r}(s)}{\mathrm{d} s}=\left(\frac{\mathrm{d} r_{\|}(s)}{\mathrm{d} s}, \frac{\mathrm{d} r_{\perp}(s)}{\mathrm{d} s}\right)=\left(t_{\|}(s), t_{\perp}(s)\right),
$$

der in den Komponenten des Tangentenvektors parallel $\left(t_{\|}(s)\right)$ und senkrecht $\left(t_{\perp}(s)\right)$ zur $x$-Achse aufgeteilt werden kann. Die bereits in Kapitel 2.1 erwähnte Tangens-Korrelationsfunktion definiert die Persistenzlänge $L_{p}$, sie zeigt den exponentiellen Zerfall der Orientierungskorrelation mit wachsendem Abstand zwischen $s$ und $s^{\prime}$. Sie ist ein wichtiges Charakteristikum der Wormlike Chain und gegeben durch [47,79]

$$
\left\langle\boldsymbol{t}(s) \boldsymbol{t}\left(s^{\prime}\right)\right\rangle=\mathrm{e}^{-\left|s-s^{\prime}\right| / L_{p}} .
$$

Da wir von Ketten mit einer hohen Persistenzlänge ausgehen, ist der Tangentenvektor $\boldsymbol{t}(s)$ nahezu parallel zur $x$-Achse ausgerichtet und die Kettenauslenkungen bezüglich der $y$-Richtung können als klein angesehen werden 13,64. So gilt die Näherung

$$
\left|\frac{\mathrm{d} \boldsymbol{t}}{\mathrm{d} s}\right|^{2}=\left(\frac{\mathrm{d} t_{\|}}{\mathrm{d} s}\right)^{2}+\left(\frac{\mathrm{d} t_{\perp}}{\mathrm{d} s}\right)^{2} \approx\left(\frac{\mathrm{d} t_{\perp}}{\mathrm{d} s}\right)^{2}
$$

Setzen wir die Approximation (2.9) in 2.6) ein und bedenken, dass im zweidimensionalen Raum $r_{\perp}(s)=y(s)$ gilt, so erhalten wir den Hamiltonian des Wormlike ChainModells in der Weakly Bending-Näherung, die von J. F. Marko und E. D. Siggia eingeführt wurde [13, 87]:

$$
\mathcal{H}[y(s)]=\frac{\kappa}{2} \int_{0}^{L} \mathrm{~d} s\left(\frac{\mathrm{d}^{2} y(s)}{\mathrm{d} s^{2}}\right)^{2} .
$$

Der Hamiltonian der elastischen Biegeenerige in der Weakly Bending-Näherung 2.10) dient als Grundlage für die weitere numerische Behandlung der Polymerketten im Rahmen der statistischen Polymerphysik (siehe Kapitel 2.5).

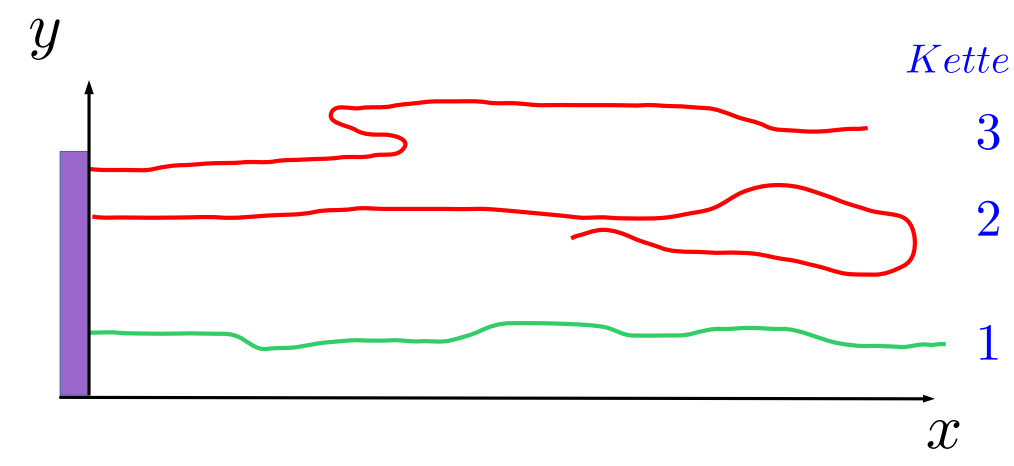

Abbildung 2.3.: Veranschaulichung von Beispielen einer erlaubten (Kette 1) und zweier verbotenen Kettenkonformationen (Kette 2 und Kette 3)

Im Modell sind die zu analysierenden Polymerketten mit einer hinreichend hohen Biegesteifigkeit $\kappa$ ausgestattet, das heißt, die Persistenzlänge $L_{p}$ der Ketten ist größer als 
deren Konturlänge $L_{c}$ : $L_{p}>L_{c}$. Eine Verknäuelung oder Schlaufenbildung der Ketten ist im Rahmen unseres Modells nicht vorgesehen, stattdessen existiert eine axiale Ausrichtung in positiver $x$-Richtung (siehe Abbildung (2.3)).

\subsection{Ein Coarse Graining-Modell einer Polymerbürste}

In diesem Kapitel beschreiben wir unser Coarse Graining-Modell einer Polymerbürste, welches wir später mittels Monte-Carlo-Simulationen studieren werden.

Wie bereits erläutert, sind wir bei den Simulationsmessungen bestrebt, den Fokus auf die Eigenschaften und Phänomene der Polymerketten auf einer mesoskopischen Längenskala zu legen und mikroskopische Details wie den molekularen Aufbau einer Monomereinheit zu vernachlässigen. Aus diesem Grunde liegt es nahe, zur Modellierung der Polymerketten ein Gittermodell einzuführen. Dieses hat sich bereits vielfach zur numerischen Untersuchung von Makromolekülen bewährt [32,39,64. Allerdings wird dabei in der Regel ein periodisches, zweidimensionales Gitter verwendet, bei dem die Monomere stets auf den Gitterpunkten liegen und daher die Bindungslänge zwischen zwei benachbarten Monomeren stets konstant und identisch mit der Gitterkonstanten ist. In unseren Simulationen benutzen wir hingegen für jede einzelne Kette ein modifiziertes, eindimensionales Gitter, bei welchem, wie unten noch genauer beschrieben wird, jeder Gitterplatz aus einem Intervall anstelle eines festen Koordinatenpunktes besteht.

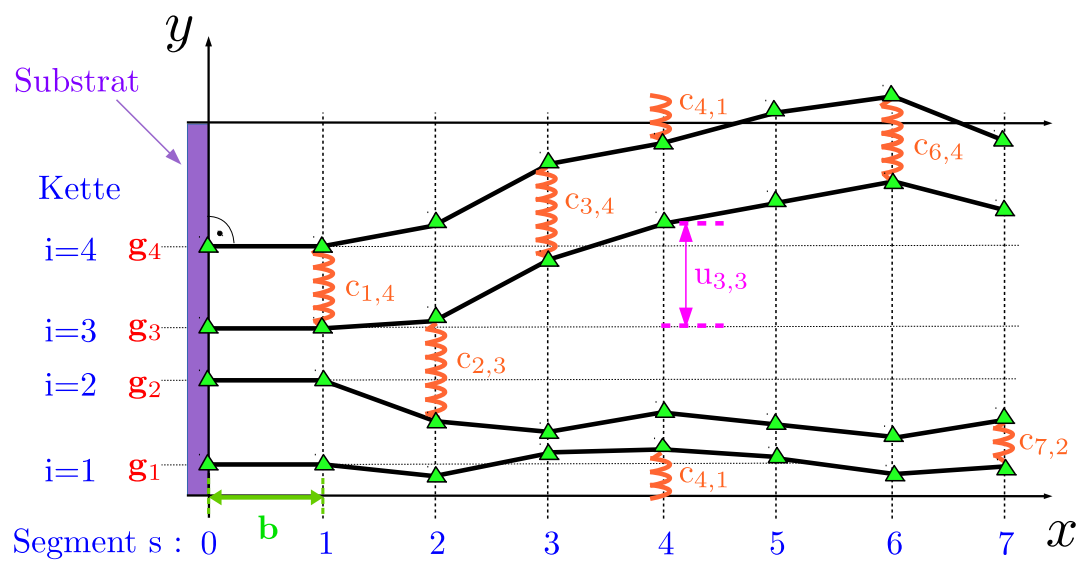

Abbildung 2.4.: Schematische Darstellung des Modells mit $N_{Y}=4$ Ketten und $(L+1)=8$ Segmenten je Kette. Außerdem sind $R=6$ Crosslinks enthalten.

Das Simulationsmodell ist aus den folgenden Komponenten zusammengesetzt (siehe Abbildung (2.4)):

- einer ebenen, eindimensionalen Substratoberfläche der Länge $H_{Y}$, sie wird beschrieben durch ein halboffenes Intervall $\mathcal{G}:=\left[0, H_{Y}\right)$ auf der $y$-Achse 
- einer festen Anzahl von $N_{Y}$ identischen, linearen Polymerketten, die aus jeweils $(L+1)$ Segmenten und aus $L$ Bindegliedern, den Bonds, bestehen

- einer festen Anzahl von $R$ Crosslinks, die durch ein Hooksches Federpotential gekennzeichnet sind.

Jede Kette wird mit Hilfe eines Kettenindexes $i=1,2, \ldots, N_{Y}$ nummeriert. Die Ketten sind auf einer ebenen Substratoberfläche rechtwinklig und punktförmig aufgepfropft, die Verknüpfungsstellen $g_{i} \in \mathcal{G}$ der Ketten mit der Substratoberfläche werden auch als Grafting-Points bezeichnet.

Alle $N_{Y}$ Grafting-Points sind voneinander verschieden, das heißt, es existiert immer ein positiver Abstand zwischen den Grafting-Points unterschiedlicher Ketten. Wir legen fest, dass der mittlere Grafting-Abstand $\left\langle d_{\mathrm{Gr}}\right\rangle$ zwischen zwei benachbarten GraftingPoints auf Eins festgelegt wird, das heißt, es gilt für die Länge der Substratoberfläche $H_{Y}=\left\langle d_{\mathrm{Gr}}\right\rangle \cdot N_{Y}=N_{Y}$. Die Ketten sind außerdem geordnet, die Indizes verlaufen also aufeinanderfolgend von der untersten zur obersten Kette, so dass für die Menge der Grafting-Points gilt:

$$
0 \leq g_{1}<g_{2}<\ldots<g_{N_{Y}}<N_{Y} \quad \text { mit } \quad g_{i} \in \mathcal{G} ; i=1,2, \ldots, N_{Y} .
$$

In unserem Modell sind die Polymerketten entlang der $x$-Achse ausgerichtet und werden ausschließlich senkrecht zur axialen Ausrichtung, das heißt parallel zur $y$-Achse ausgelenkt. Diese transversalen Auslenkungen entsprechen den thermischen Fluktuationen der Polymere und bestimmen deren Kettenkonformation.

Die $(L+1)$ strukturbildenden Grundeinheiten einer Polymerkette, die Segmente, werden auf ein eindimensionales und reguläres Gitter $\left(x_{0}, x_{1}, \ldots, x_{L}\right)$ auf dem Intervall $\mathcal{P}:=[0, L]$ der $x$-Achse projiziert, mit den Gitterkoordinaten $x_{s}=b \cdot s$, der Segmentkennzahl $s=0,1, \ldots, L$ und der Gitterkonstanten $b$. Wir erhalten folglich für alle $N_{Y}$ Ketten jeweils ein eindimensionales Gitter mit $(L+1)$ Gitterplätzen. Das gesamte Modell weist somit insgesamt $N_{\text {Seg }}:=N_{Y} \cdot(L+1)$ Segmente bzw. Gitterplätze auf.

Die Menge der Gitterpunkte bezeichnen wir mit $S:=\left\{x_{s} \in \mathcal{P} \mid x_{s}=b \cdot s, s=0,1, \ldots, L\right\}$. Jeder Gitterplatz wird somit durch genau ein Segment belegt und durch die Zahl $s$ eindeutig gekennzeichnet. Mit $b=1$ ist die Projektionslänge der gesamten Kette mit $L \cdot b=L$ festgelegt. Da diese eindimensionalen Gitterplätze nebeneinander bzw. parallel zueinander angeordnet sind, sprechen wir hierbei von einem $(1+1)$-dimensionalen Gittermodell.

Wir beschreiben die Auslenkungen der Kette $i$ an der Stelle $x_{s}=s$ (das heißt bei der Gitterkoordinate $\left.x_{s}\right)$ mit $u_{i}\left(x_{s}\right)=: u_{s, i}$. Die Angabe der Kettenposition an der Stelle des Segments $s$ setzt sich aus der Grafting-Position $g_{i}$ und der Kettenauslenkung bei $x_{s}$ zusammen und wir schreiben daher für die $y$-Komponente

$$
y_{i}\left(x_{s}\right)=: y_{s, i}=g_{i}+u_{s, i}
$$

Die Segmentkoordinaten im Modellsystem werden durch die Positionsvektoren $\boldsymbol{r}_{s, i}$ (siehe Ausdruck (2.1) ) angegeben, die sich aus dem Positionsvektor der Grafting-Points $\boldsymbol{g}_{i}$ 
und dem der Segmentauslenkung $\boldsymbol{u}_{s, i}$ ergeben, und lauten in unserem Koordinatensystem

$$
\boldsymbol{r}_{s, i}=\boldsymbol{g}_{i}+\boldsymbol{u}_{s, i}=\left(0, g_{i}\right)+\left(b \cdot s, u_{s, i}\right)=\left(b \cdot s, y_{s, i}\right) .
$$

Daraus ergeben sich auch unmittelbar die Koordinaten der Bond-Vektoren, die vektoriell die stabartigen Bindeglieder zweier aufeinanderfolgender Monomere $s$ und $(s+1)$ derselben Kette $i$ repräsentieren: $\boldsymbol{t}_{s, i}=\left(b, u_{s+1, i}-u_{s, i}\right)$.

Wichtig zu erwähnen ist hierbei, dass zwischen den einzelnen Ketten keine Molekülverbindungen, sondern lediglich Verknüpfungen über Crosslinks existieren können (siehe unten).

Die vollständige Information über die Struktur des Systems ergibt sich aus der Anordnung der einzelnen Segmente, das heißt durch die Angabe der $N_{Y} \cdot L$ Bond-Vektoren $\boldsymbol{t}_{s, i}$ bzw. der $N_{Y} \cdot(L+1)$ Positionsvektoren $\boldsymbol{r}_{s, i}$.

Bei einem periodischen, zweidimensionalen Gittermodell, wie es zum Beispiel in 39 beschrieben wird, ist die Länge der Bond-Vektoren mit der Gitterkonstanten identisch $\left(\left|\boldsymbol{t}_{s, i}\right|=b\right)$. Die Segmente einer Polymerkette können dabei auf benachbarte Gitterplätze ausweichen und alle freien Gitterplätze sind theoretisch für jedes Segment zugänglich. Allerdings sind in unserem Fall mit Segmentauslenkungen $u_{s, i}$ keine Gitterplatzwechsel verbunden. Die Segmente bleiben immer an ihrem ursprünglichen Gitterplatz, der streng genommen ein eindimensionales Intervall der Länge $N_{Y}$ ist. Folglich ist die Länge der Bond-Vektoren abhängig von der Auslenkung der Segmente. Es gilt für den Betrag eines Bond-Vektors: $\left|\boldsymbol{t}_{s, i}\right|=\sqrt{b^{2}+\left(u_{s, i}-u_{s-1, i}\right)^{2}}$. Wir gehen allerdings von einer für Ketten mit einer hohen Biegesteifigkeit vernachlässigbaren Abweichung aus und berücksichtigen lediglich die auf die $x$-Achse projizierte Länge, die mit der Gitterkonstanten $b$ identisch ist.

Im hier zu analysierenden Modell ist dementsprechend die exakte Konturlänge $L_{c}$ jeder Kette des Modells unerheblich, da wir ausschließlich deren Projektion auf die $x$-Achse betrachten, das heißt $L \leq L_{c}$.

Aus der Tatsache, dass Segmente niemals den Gitterplatz wechseln und zu allen Zeiten eindeutig mit ein und demselben Gitterplatz assoziiert werden können, lässt sich ableiten, dass sowohl Doppelbesetzungen als auch leere Gitterplätze nicht erlaubt sind.

Alle beweglichen Segmente führen lediglich thermische Bewegungen auf ihrem Platz aus, das heißt im Rahmen unseres Modells sind ausschließlich eindimensionale Fluktuationsbewegungen um die jeweilige Ruhelage senkrecht zur Gitterachse möglich, das heißt der Freiheitsgrad eines beweglichen Segments ist gleich Eins. Eine Polymerkette nimmt die Ruhelage ein, falls sie exakt senkrecht zur Substratoberfläche oder parallel zur $x$-Achse ausgerichtet ist, das bedeutet, dass in diesem Fall alle Segmentauslenkungen gleich Null sind. Im Gegensatz zu den diskreten Gitterpositionen $x_{s} \in S$ ist für die thermischen Segmentbewegungen und den damit assoziierten Auslenkungen ein kontinuierlicher Wertebereich erlaubt, dabei gilt aufgrund von periodischen Randbedingungen $u_{s, i} \in \mathcal{G}$ (siehe unten).

Wir bezeichnen im Folgenden mit $(s, i)$ die $(L+1) \cdot N_{Y}=: N_{\text {Seg }}$ Gitterkoordinaten des gesamten Systems, mit den Indizes $s=0,1, \ldots, L$ und $i=1,2, \ldots, N_{Y}$. Eine weitere wichtige Eigenschaft, durch die sich das Modell auszeichnet, ist die Verwendung 
von periodischen Randbedingungen bezüglich der $y$-Richtung. Es gilt 17, 91, 111: $\left(s, N_{Y}+1\right)=(s, 1) \forall s=0,1, \ldots, L$. Zur einfachen Veranschaulichung der periodischen Randbedingungen in $y$-Richtung kann man sich die Polymerketten aufgereiht auf der Mantelfäche eines Zylinders vorstellen.

Die periodischen Randbedingungen sorgen dafür, dass sich die gesamte Physik innerhalb eines beschränkten, quaderförmigen Gebiets $\mathcal{P} \times \mathcal{G} \subset \mathbb{R}^{2}$, der sogenannten Simulationsbox, abspielt. Streng genommen spielt sich die Physik sogar ausschließlich auf den diskreten Gitterpunkten $x_{s} \in S$ ab, so dass für die Simulationsberechnungen lediglich die beschränkte Punktmenge

$$
\Omega:=S \times \mathcal{G} \subset \mathbb{R}^{2}
$$

relevant ist.

Alle Segmentpositionen $\tilde{y}_{s, i}$, die zu Werten außerhalb des Intervalls $\mathcal{G}$ führen, treten am gegenüberliegenden Rand der Simulationsbox wieder ein. Einer Segmentposition außerhalb dieses Intervalls werden dann neue Koordinaten $y_{s, i}$ zugeordnet (es wird vorausgesetzt, dass die Auslenkungen niemals größer als $N_{Y}$ sind):

$$
y_{s, i}= \begin{cases}\tilde{y}_{s, i}-N_{Y}, & \text { falls } N_{Y} \leq \tilde{y}_{s, i}<2 N_{Y} \\ \tilde{y}_{s, i}+N_{Y}, & \text { falls }-N_{Y} \leq \tilde{y}_{s, i}<0\end{cases}
$$

Bezüglich der Segmentauslenkung gibt es keine Vorzugsrichtung. Somit sind die Auslenkungen in positiver und negativer Richtung gleichwahrscheinlich und $u_{s, i}$ kann sowohl positive auch als negative Werte annehmen.

Um der eingangs genannten Forderung nach senkrecht zur Substratoberfläche aufgepfropften Ketten gerecht zu werden, sind die beiden ersten Segmente, in unserer Notation das nullte und das erste Segment jeder Kette starr, das heißt, es gilt die Zwangsbedingung:

$$
u_{0, i}=u_{1, i} \equiv 0 \quad \forall i=1,2 \ldots, N_{Y} .
$$

Der erste Bond-Vektor jeder Kette, der die Verbindung zwischen den ersten beiden Segmenten markiert, steht somit rechtwinklig auf der Oberfläche bzw. ist exakt parallel zur $x$-Achse ausgerichtet: $\boldsymbol{r}_{0, i}=(b, 0)$. Offensichtlich besitzt das System in $x$-Richtung keine periodischen Randbedingungen, da die Segmente am Kettenanfang fest, am Kettenende dagegen frei beweglich sind. Die in (2.16) angegebene Bedingung legt die Zahl der beweglichen Segmente und damit die Freiheitsgrade der Polymersegmente auf $(L-1) \cdot N_{Y}$ fest.

Die einzelnen, eindimensionalen Gitter sind als unabhängig voneinander zu betrachten. Allerdings bestehen Wechselwirkungen zwischen den Ketten über die Crosslinks, die Segmente zwischen verschiedenen Ketten verbinden. Eine weitere Möglichkeit der Interaktion zwischen Polymerketten kann über die Excluded Volume-Wechselwirkung bestehen. Dieses Konzept wurde von W. Kuhn und P. J. Flory eingeführt 23, 25, 32, 38, Das bedeutet, das sich gegebenenfalls aufgrund dieser Excluded Volume-Wechselwirkung zwei Segmente in der freien Bewegung behindern (siehe Kapitel 2.8). 


\subsection{Biegeenergie $\mathcal{H}_{\kappa}$}

Der Hamiltonian der Biegeenergie (2.10) geht von einer kontinuierlichen Raumkurve $y(s)$ des Polymers aus. Aufgrund der Diskretisierung der Ketten ist deshalb eine Modifizierung des Energiefunktionals $\mathcal{H}[y(s)]$ notwendig, das heißt, wir berücksichtigen daher eine diskrete Krümmung [76].

Mit Hilfe der Methode der finiten Differenzen lässt sich die Ableitung 2. Ordnung approximativ berechnen 18,102. Unter Verwendung des zentralen Differenzenquotienten an der Stelle $x$ erhält man

$$
\frac{\mathrm{d}^{2} y(x)}{\mathrm{d} x^{2}}=\frac{y(x+b)-2 y(x)+y(x-b)}{b^{2}}+\mathcal{O}\left(b^{2}\right) .
$$

Die Schrittbreite entspricht der Gitterkonstanten $b$. Zur Approximation der Krümmung am Gitterpunkt $x_{s}$ tragen gemäß (2.17) die Werte von drei aufeinanderfolgenden Gitterpunkten $x_{s-1}, x_{s}, x_{s+1} ; s=1,2, \ldots,(L-1)$ bei. Anstelle des Integrals in (2.10) steht für jede Kette die Summe über $(L-1)$ diskrete Krümmungswerte. Die beiden äußeren Gitterpunkte $x_{0}$ und $x_{L}$ liefern keinen Beitrag zur Biegeenergie.

Wegen (2.12) heben sich die Grafting-Positionen in (2.17) gegenseitig auf und es kommt ausschließlich auf die Auslenkungen der beweglichen Kettensegmente an. Wir erhalten durch numerische Integration für den Term der Biegeenergie

$$
\mathcal{H}_{\kappa}=\sum_{i=1}^{N_{Y}} \sum_{s=1}^{L-1} \mathcal{H}_{\kappa}[(s, i)]=\frac{\kappa}{2} \sum_{i=1}^{N_{Y}} \sum_{s=1}^{L-1}\left(u_{s-1, i}-2 u_{s, i}+u_{s+1, i}\right)^{2},
$$

$s=1,2, \ldots,(L-1)$, mit der lokalen Biegeenergie $\mathcal{H}_{\kappa}[(s, i)]=\frac{\kappa}{2} \cdot\left(u_{s-1, i}-2 u_{s, i}+u_{s+1, i}\right)^{2}$ an der Position mit den Koordinaten $(s, i)$. Die auf diese Weise genäherte Biegeenergie des Gesamtsystems ist somit durch die $N_{Y} \cdot(L-1)$ Auslenkungskoordinaten $u_{s, i}$ und den Parameter der Biegesteifigkeit $\kappa$ eindeutig bestimmt und wird in dieser Form vom Programm verwendet.

\subsection{Crosslinkenergie $\mathcal{H}_{\text {xlink }}$}

Bisher haben wir uns nur mit der Beschreibung der Polymerketten und deren Verknüpfungen mit der ebenen Fläche befasst. Allerdings haben wir mit den Crosslinks einen wesentlichen und wichtigen Bestandteil unseres Modells bisher außen vorgelassen, der jedoch für die Simulation unerlässlich ist.

Unter einem Crosslink verstehen wir ein Verbindungsglied, das zwei Polymerstränge miteinander verknüpft. Dieser steht für die in Kapitel 2.1 erläuterten actin-bindenden Proteine. Die bereits eingeführten und durch die Bond-Vektoren dargestellten Bindungen zwischen benachbarten Segmenten derselben Kette sind wie beschrieben starr und haben in guter Näherung eine konstante Länge. Im Gegensatz dazu besitzt ein Crosslink ein harmonisches Federpotential und verknüpft stets genau zwei Segmente von zwei unterschiedlichen, nebeneinanderliegenden Ketten. Die Crosslinks werden durch Hooksche Federn mit einer Crosslink-Stärke $\alpha$ modelliert. 
Zwar sind stets zwei Segmente durch genau einen Crosslink miteinander verbunden, zur exakten Kennzeichnung des Crosslinks allerdings werden per Konvention die Indizes des oberen Segments zugeordnet. Für die Crosslink-Energie eines Crosslinks mit den Koordinaten $(s, i)$, der die beiden Segmente $(s, i-1)$ und $(s, i)$ miteinander verbindet, schreiben wir daher

$$
\mathcal{H}_{\text {xlink }}[(s, i)]=\alpha \cdot\left(d_{s, i}\right)^{2}, \quad d_{s, i}:=\left\{\begin{array}{ll}
\left|\left(y_{s, 1}-y_{s, N_{Y}}\right)+N_{Y}\right| & , \text { falls } i=1 \\
\left|y_{s, i}-y_{s, i-1}\right| & , \text { sonst }
\end{array},\right.
$$

mit dem Crosslink-Abstand $d_{s, i}$.

Im Rahmen unseres Modells gibt es genaue Vorgaben für Crosslinks hinsichtlich der Verknüpfung zweier Ketten:

- Die Crosslinks sind reversibel

- Die Anzahl der Crosslinks ist konstant

- Crosslinks setzen ausschließlich an den Segmenten an, nicht jedoch an den Bonds.

- Es werden immer genau zwei Segmente miteinander verknüpft.

- Die beiden über einen Crosslink verbundenen Segmente haben stets den selben Segmentindex $s$ und müssen benachbarten Ketten angehören.

- Jeder Gitterplatz kann höchstens von einem Crosslink eingenommen werden. Das bedeutet, dass an jedem Segment maximal zwei Crosslinks andocken können.

Aufgrund der Reversibilität der Crosslink-Verbindungen können diese thermisch bedingt wieder aufbrechen und an einer anderen Stelle neu entstehen (Detailed Balance) [91,111. Die Crosslinkzahlerhaltung macht es zwingend erforderlich, dass mit dem Lösen einer Verbindung stets die Entstehung einer neuen Bindung an anderer Stelle erfolgt (siehe Kapitel 2.8).

Die oben genannten Vorgaben schränken die Möglichkeiten von Crosslink-Verbindungen bzw. die Freiheitsgrade des Systems ein. So stimmt gemäß unseren Forderungen trivialerweise die Anzahl der potentiellen Crosslinkplätze mit der Segmentanzahl überein. Außerdem kann das Segment $(s, i)$ via Crosslink nur mit dem Segment $(s, i+1)$ oder $(s, i-1)$ verbunden sein. Unter Berücksichtigung der periodischen Randbedingungen sind die Ketten mit $i_{1}=1$ und $i_{2}=N_{Y}$ nächste Nachbarn und folglich über Crosslinks miteinander verknüpfbar.

Im Einklang mit den obigen Vorgaben sind somit Crosslink-Verbindungen mit übernächsten Nachbarn oder weiter entfernten Ketten nicht erlaubt. Auch schräg platzierte oder offene (das heißt nur an einem einzigen Segment sitzende) Crosslinks werden in diesem Modell ausgeschlossen. Generell sind alle Crosslinks parallel zur Segmentoberfläche bzw. zur $y$-Achse ausgerichtet.

Bei der Simulation werden per Zufallsgenerator im Programmablauf $111 R$ Crosslinks auf $N_{\text {Seg }}$ Gitterplätze verteilt. Sei

$$
\mathcal{C}=\left\{\left(s_{1}, i_{1}\right),\left(s_{2}, i_{2}\right), \ldots,\left(s_{R}, i_{R}\right)\right\}
$$

die Menge der per Zufall gewählten $R$ Gitterplatz-Koordinaten der Crosslinks. Für die 
sich aus allen $R$ Crosslinks zusammengesetzte Crosslink-Energie schreiben wir schließlich:

$$
\mathcal{H}_{\text {xlink }}=\sum_{n=1}^{R} \mathcal{H}_{\text {xlink }}\left[\left(s_{n}, i_{n}\right)\right]=\alpha \cdot \sum_{n=1}^{R}\left(d_{s_{n}, i_{n}}\right)^{2} .
$$

\subsection{Parameter-Einstellungen}

Das Modell wird durch unterschiedliche Parametereigenschaften charakterisiert. Dadurch ergibt sich eine Vielzahl von Kombinationsmöglichkeiten innerhalb des Parameterraums aufgrund verschiedener, voneinander unabhängiger Parameter. Die Parameter werden zu Beginn der Simulation vom Programm eingelesen. Sie sind im Folgenden aufgeführt:

\section{Parameter 1 und 2: Anzahl der Polymerketten $N_{Y}$ und der Segmente $(L+1)$}

Die beiden Parameterwerte $N_{Y}$ und $(L+1)$ sind natürliche Zahlen, die die Systemgröße, das heißt Höhe und Breite der Simulationsbox und auch die gesamte Anzahl an Gitterplätzen $N_{\text {Seg }}:=N_{Y} \cdot(L+1)$ vorgeben.

Hierbei ist auf eine geeignete Wahl der Systemgröße zu achten. Während ein zu kleines System nur über eine unzureichende Aussagefähigkeit verfügt und mögliche Phänomene nicht sichtbar gemacht werden können, benötigt man bei einem zu großen System eine lange Equilibrierungszeit (siehe Kapitel 2.13).

\section{Parameter 3: Crosslink-Dichte $\rho$}

Mit dem Parameter $\rho$ wird die Crosslink-Dichte und damit die Anzahl der eingebrachten Crosslinks bzw. die Anzahl der durch Crosslinks belegten Gitterplätze festgelegt. Dabei ist die Anzahl der Crosslinks $R$ im Modell die aufgerundete, ganzzahlige Zahl des Produkts aus Crosslink-Dichte und Anzahl der Gitterplätze:

$$
R:=\left\lceil\rho \cdot N_{\text {Seg }}\right\rceil, \text { mit } 0 \leq \rho \leq 1,
$$

und es gilt folglich $0 \leq R \leq N_{\text {Seg. }}$. Dabei kommt es darauf an, $\rho$ geeignet zu wählen, um crosslink-abhängige Effekte in verschieden starker Ausprägung aufnehmen zu können.

\section{Parameter 4: Biegesteifigkeit $\kappa$}

Der Parameter der Biegesteifigkeit reguliert die Stärke der thermischen Fluktuationen und damit die Segmentauslenkungen. Da die Ketten in der Weakly Bending-Näherung betrachtet werden, ist $\kappa$ hinreichend groß zu wählen. Allerdings ist auch hierbei Vorsicht geboten, denn ein zu hoher Wert von $\kappa$ würde die Fluktuationsbewegungen zu stark unterdrücken. Die Biegesteifigkeit ist umgekehrt proportional zur Temperatur, das heißt, es gilt $\kappa \propto T^{-1}$. Eine Erhöhung der Biegesteifigkeit ist also gleichbedeutend mit einer Temperaturabnahme. 


\section{Parameter 5: Crosslink-Stärke $\alpha$}

Mit der in 2.19) eingeführten Crosslink-Stärke reguliert man die Federstärke bzw. die Elastizität der Crosslinks und damit auch den mittleren Abstand der miteinander verbundenen Segmente.

\section{Parameter 6: Random Graft $\mathcal{R} G r$}

Ein weiterer und wichtiger Parameter ist eine Kennzahl, die die Anordnung der Grafting-Positionen $\left\{g_{0}, \ldots, g_{N_{Y}}\right\}$ der Ketten betrifft. Diese Kennzahl $\mathcal{R} G r$ kann nur die Werte Null oder Eins annehmen und gibt an, ob äquidistante Grafting-Points gegeben sind oder ob eine per Zufall festgelegte Grafting-Konfiguration vorliegt.

- $\mathcal{R} G r=0$ : Die Ketten sind an der Oberfläche regelmäßig angeordnet, das heißt sie sind alle äquidistant mit Abstand Eins auf der Fläche aufgepfropft. So gilt

$$
\left.g_{i}\right|_{\mathcal{R} G r=0}=i-\frac{1}{2} ; \forall i=1,2, \ldots, N_{Y} .
$$

- $\mathcal{R} G r=1$ : Alle Grafting-Positionen werden per Zufallsgenerator auf dem Grafting-Intervall $\mathcal{G}=\left[0, N_{Y}\right)$ festgelegt. Dabei spielt die Intervallbreite $\Delta$ als Ordnungsmaß der Grafting-Konfiguration eine wichtige Rolle (siehe unten). Ein Sortier-Algorithmus ordnet die Ketten anschließend, so dass die in 2.11) geforderte Reihenfolge gegeben ist.

Das Programm erzeugt eine gleichverteilte Zufallszahl $\xi$ auf dem halboffenen Einheitsintervall $[0,1)$. Wir betrachten zum Beispiel im Falle von völlig zufällig angeordneten Grafting-Points $g_{i}$ deren Verteilung als gleichverteilte Zufallsvariable auf dem Intervall $\mathcal{G}$ mit der Wahrscheinlichkeitsdichtefunktion 22

$$
f\left(g_{i}\right)=\left\{\begin{array}{ll}
\frac{1}{N_{Y}} & \text { falls } 0 \leq g_{i}<N_{Y} \\
0 & \text { sonst }
\end{array},\right.
$$

das heißt es werden $N_{Y}$ Zufallszahlen $\xi_{i} ; i=1,2, \ldots, N_{Y}$ generiert und so geordnet, dass $\xi_{1}<\xi_{2}<\ldots<\xi_{N_{Y}}$ gilt. Schließlich wird die Grafting-Position durch die Vorschrift

$$
g_{i}=\xi_{i} \cdot N_{Y} ; i=1,2, \ldots, N_{Y}
$$

festgelegt.

\section{Parameter 7: Intervallbreite $\Delta$}

Wie später noch ausführlich beschrieben wird, ist in manchen Fällen bezüglich des Ordnungsgrades ein kontinuierlicher Übergang von streng angeordneten Ketten $(\mathcal{R} G r=0)$ hin zu komplett zufällig angeordneten Ketten sinnvoll. Aus diesem Grund führen wir mit $\Delta$ einen Parameter ein, der eine Intervallbreite auf $\mathcal{G}$ festlegt. Ausgehend von der perfekt geordneten, äquidistanten Anordnung erlauben wir jeder Kette eine Abweichung mit einer Breite von $\Delta$ um seine Ausgangsposition. Mit der Zufallszahl $\xi_{i} \in[0,1)$ 
gilt für die Grafting-Positionen

$$
g_{i}(\Delta)=\underbrace{i-\frac{1}{2}}_{g_{i} \mid \mathcal{R} G r=0}+\left(\frac{1}{2}-\xi_{i}\right) \cdot \Delta,
$$

und unter Berücksichtigung der periodischen Randbedingungen 2.15 werden die Grafting-Positionen der Ketten mit Hilfe eines Sortier-Algorithmus in die richtige Reihenfolge gebracht (vergleiche (2.11)) .

Während $\Delta=0$ offensichtlich zum Spezialfall $\mathcal{R} G r=0$ führt, wird im Falle $\Delta=N_{Y}$ wegen (2.25) bei der Positionsfestlegung für jeden Punkt $g_{i}$ das ganze Intervall $\mathcal{G}$ zugänglich. Mit der Variation von $\Delta \in\left[0, N_{Y}\right]$ kann mit der damit verbundenen kontinuierlichen Veränderung der Intervallbreite Einfluss auf die Ordnung der Grafting-Positionen genommen werden.

Wie man leicht erkennen kann, wird der Grad der Unordnung schnell mit wachsendem $\Delta$ zunehmen. Dabei muss zwischen der Unordnung einer einzelnen Grafting-Konfiguration und der Mittelung über mehrere Konfigurationen unter Beibehaltung eines festen Wertes für $\Delta$ unterschieden werden (siehe Kapitel 2.9). Wir verwenden im Folgenden die Bezeichnung $\mathcal{R} G r=1$ für alle von der äquidistanten Grafting-Konfiguration abweichenden Zufallsanordnungen, das heißt $\forall \Delta>0$ gilt $\mathcal{R} G r=1$.

\section{Parameter 8: Excluded Volume Ex-Vol}

Außer der Verknüpfung mit Crosslinks gibt es noch eine weitere Möglichkeit der Polymer-Polymer-Wechselwirkung, nämlich über den Excluded Volume-Effekt. Die $N_{Y}$ Polymerketten befinden sich jeweils auf ihrem eigenen Gitter und können ohne die Excluded Volume-Wechselwirkung (das heißt für $E x$-Vol $=0$ ) nicht miteinander in Wechselwirkung treten. Das bedeutet, dass sich die Segmente ungehindert aneinander vorbeibewegen können und daher die Möglichkeit der Kreuzung oder Überlappung der Ketten besteht.

Diese freie Fluktuationsbewegung kann mit Hilfe des Excluded Volume-Parameters unterdrückt werden. Wird beim Start der Simulation der Excluded Volume-Effekt aktiviert (das heißt, gilt $E x-V o l=1$ ), so können sich die Segmente desselben Segmentindexes $s$ nicht mehr aneinander vorbeibewegen oder durchdringen, da alle auf derselben Geraden mit $x_{s}=b \cdot s$ liegen. Die Segmente stoßen sich wie harte Kugeln ab und ein Aneinander-Vorbeikommen ist im eindimensionalen Raum nicht möglich.

Das standardmäßig verwendete Potential zur Beschreibung der Wechselwirkung von zwei neutralen Atomen ist das Lennard-Jones Potential [63], bei dem bei großen Entfernungen der anziehende Anteil, bei kleinen Entfernungen hingegen die Abstoßung überwiegt. In unserem Modell wird jedoch das Potential vereinfacht durch eine unendlich hohe Potentialbarriere. So gibt es weder langreichweitige noch attraktive Kräfte zwischen den Segmenten, sondern lediglich eine repulsive Kraft der als punktförmig angenommenen Segmente bei Überlappung. Das effektive Wechselwirkungspotential zwischen zwei Segmenten $(s, i)$ und $(s, i-1)$ kann dementsprechend beschrieben wer- 
den durch

$$
\mathcal{U}_{\text {ex }}=\left\{\begin{array}{lll}
\infty & \text { wenn } & d_{s, i}=0 \\
0 & \text { wenn } & d_{s, i}>0
\end{array}\right.
$$

\section{Anmerkung zu Parameter-Angabe}

Bei den im weiteren Verlauf auftretenden Darstellungen der Simulationsdaten werden die essentiellen Parameter-Werte in den Abbildungen angegeben, so dass stets die relevanten Parameter ersichtlich sind.

\subsection{Ordnungsmaße für die Grafting-Konfiguration}

Hier geben wir die Definitionen der uns interessierenden Schlüsselobservablen an, die später in den Monte-Carlo-Simulationen gemessen werden. Insbesondere richten wir unser Interesse darauf, wie man die Ordnung der Grafting-Konfiguration ermitteln kann.

Um ein Maß für die Ordnung der Grafting-Konfiguration $\left\{g_{0}, \ldots, g_{N_{Y}}\right\}$ zu ermitteln, gibt es zwei geeignete Möglichkeiten:

\section{Der statische Strukturfaktor $S(s, q)$}

Der statische Strukturfaktor ist eine wichtige Größe zur Bestimmung der Polymerstruktur [46], und kann als ein Ordnungsmaß für die Grafting-Konfiguration verwendet werden. In Kapitel 2.14 werden wir im Zusammenhang mit der Auswertung von strukurellen Informationen der Ketten auf ihn zurückkommen. Im Allgemeinen sind wir am Strukturfaktor bei einem festen Segmentindex $s$ interessiert, das heißt, relevant zur Bestimmung von $S(s, q)$ sind alle $N_{Y}$ Segmentpositionen entlang der $y$-Achse mit demselben Segmentindex $s$. So erhalten wir gemäß der Anzahl der Segmente $(L+1)$ Strukturfunktionen.

Der statische Strukturfaktor bezüglich des Segments $s$ wird definiert durch 46, 48,

$$
S(s, q):=\left\langle\frac{1}{N_{Y}} \cdot\left|\sum_{i=1}^{N_{Y}} \exp \left(\mathrm{i} q y_{s, i}\right)\right|^{2}\right\rangle,
$$

mit dem Wellenvektor

$$
q=\frac{2 \pi}{N_{Y}} \cdot n, n=1,2, \ldots, n_{\max },
$$

und der geeignet gewählten maximalen Quantenzahl $n_{\max }=2 N_{Y}$. Mit $\langle Q\rangle$ wird der zeitliche Mittelwert der Größe $Q$ bezeichnet [48] und ersetzt den thermischen Mittelwert:

$$
\langle Q\rangle:=\lim _{t \rightarrow \infty} \frac{1}{t} \int_{0}^{t} Q\left(t^{\prime}\right) \mathrm{d} t^{\prime} .
$$


Die Grafting-Konfiguration an der Substratoberfläche wird zu Beginn eines jeden Simulationslaufs (Runs) einmalig festgelegt und ist für diesen Lauf zeitlich konstant. Diese Konfiguration der $N_{Y}$ verschiedenen Grafting-Positionen wird als Quenched Disorder angesehen [14, 45, 60]. So ist für die ersten beiden festen Segmente $s \in\{0,1\}$ zur Bestimmung des statischen Strukturfaktors keine zeitliche Mittelung nötig und (2.27) vereinfacht sich mit $2.16 \mathrm{zu}$

$$
S(0, q)=S(1, q)=\frac{1}{N_{Y}} \cdot\left|\sum_{i=1}^{N_{Y}} \exp \left(\mathrm{i} q g_{i}\right)\right|^{2} .
$$

Die Grafting-Positionen hängen von der als Seed bezeichneten Zahl ab, mit der der Zufallsgenerator beim Programmstart gespeist wird [91,111. Unterschiedliche Seeds, die in den einzelnen, voneinander unabhängigen Runs erzeugt werden, haben konsequenterweise auch unterschiedliche Grafting-Positionen zur Folge. Sofern es für die Beschreibung relevant ist, geben wir zur Identifizierung eines Simulations-Runs den Index, die sogenannte Run-ID $r=1,2, \ldots, r_{\max }$, an. Damit definieren wir den Quenched Disorder-Mittelwert einer Größe $Q$ aus den Werten von Einzelmessungen $Q_{r} ; r=1,2, \ldots, r_{\max }$, die aus $r_{\max }$ unterschiedlichen, voneinander unabhängigen Simulationsläufen ermittelt wurden:

$$
\left.Q\right|_{\mathrm{qd}}:=\frac{1}{r_{\max }} \cdot \sum_{r=1}^{r_{\max }} Q_{r}
$$

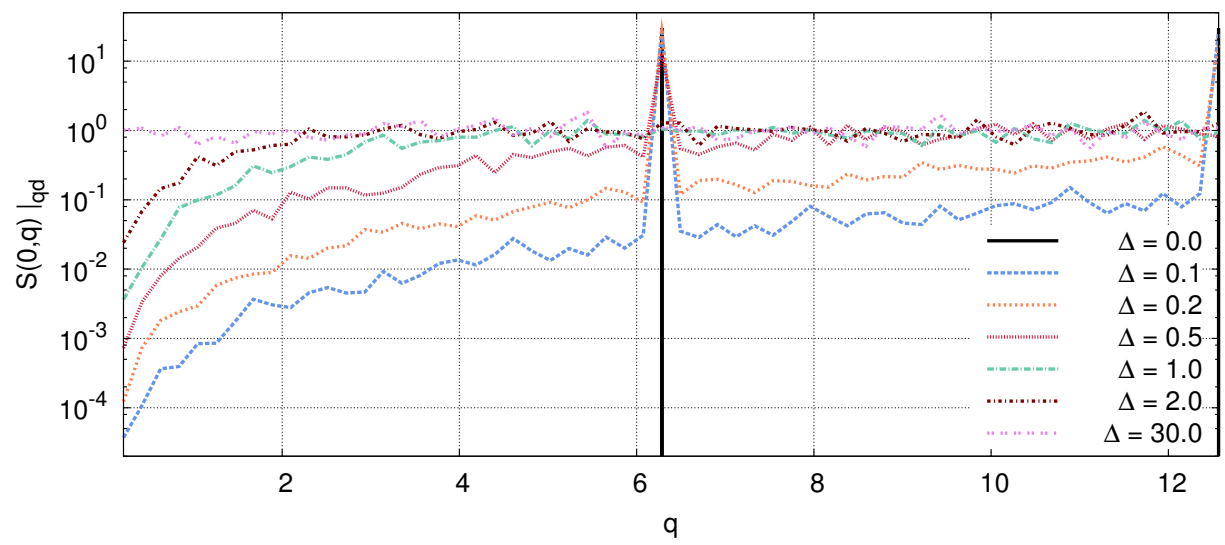

Abbildung 2.5.: Strukturfaktor $\left.S(0, q)\right|_{\text {qd }}$ an der Substratoberfläche als Funktion der Wellenzahl $q$ für $N_{Y}=30$ Ketten und $r_{\max }=20$ Runs für verschiedene Werte der Intervallbreite $\Delta$.

Ein repräsentatives Ordnungsmaß in Abhängigkeit der Intervallbreite $\Delta$ erreicht man durch die Mittelung des Strukturfaktors über viele Runs mit jeweils unterschiedlichen Seeds bzw. Grafting-Konfigurationen. Sei $\left\{g_{0}^{r}, \ldots, g_{N_{Y}}^{r}\right\}$ die Konfiguration des $r$-ten Laufs und $S_{r}(s, q)$ der dazugehörige Strukturfaktor, dann erhalten wir gemäß (2.31) für den Quenched Disorder-Mittelwert des Strukturfaktors:

$$
\left.S(s, q)\right|_{\mathrm{qd}}:=\frac{1}{r_{\max }} \cdot \sum_{r=1}^{r_{\max }} S_{r}(s, q) .
$$


In Abbildung (2.5) ist der Quenched Disorder-Mittelwert des Strukturfaktors für die Substratoberfläche $\left.S(0, q)\right|_{\text {qd }}$ für unterschiedliche $\Delta$-Werte in Abhängigkeit von der Wellenzahl $q$ aufgetragen. Der ideale Ordnungszustand ist bei äquidistant gegrafteten Polymerketten erreicht $(\Delta=0)$. Der Strukturfaktor spiegelt dies durch eine periodische Abfolge von äquidistanten $\delta$-Peaks mit einem Abstand von $2 \pi$ wider. Eine kontinuierliche Vergrößerung der Intervallbreite $\Delta$ hat bei einer hinreichend hohen Statistik bzw. einer hohen Anzahl verschiedener Grafting-Konfigurationen einen wachsenden Untergrund zwischen den Peaks zur Folge und zeigt damit die wachsende Unordnung der Grafting-Positionen. Im Grenzfall maximaler Unordnung lassen sich keine Korrelationen erkennen und $\left.S(0, q)\right|_{\text {qd }}$ pendelt sich unabhängig von der Wellenzahl $q$ bei einem Wert von circa Eins ein.

\section{Anmerkung zum Quenched Disorder-Mittelwert}

Sofern es explizit nicht anders angegeben ist, handelt es sich bei den kommenden Quenched Disorder-Mittelwerten um eine Mittelung aus $r_{\max }=20$ verschiedenen Runs mit ebenso vielen unterschiedlichen Grafting-Positionen.

\section{Der empirische Korrelationskoeffizient $K\left(g^{0}, g^{1}\right)$}

Als Maß für die Stärke des linearen Zusammenhangs zwischen der gleichmäßigen Grafting-Konfiguration der Ketten $\left.\left\{g_{0}, \ldots, g_{N_{Y}}\right\}\right|_{\mathcal{R} G r=0}$ und einer unregelmäßigen, zufälligen Kettenanordnung $\left.\left\{g_{0}, \ldots, g_{N_{Y}}\right\}\right|_{\mathcal{R} G r=1}$ sind wir an der Bestimmung des empirischen Korrelationskoeffizienten nach A. Bravais und K. Pearson interessiert [22,73]. Auf diese Weise kann ein weiteres Ordnungsmaß festgelegt werden. Es veranschaulicht, wie schnell die Ordnung mit wachsendem $\Delta$ sinkt. Für die Messung der linearen Korre-

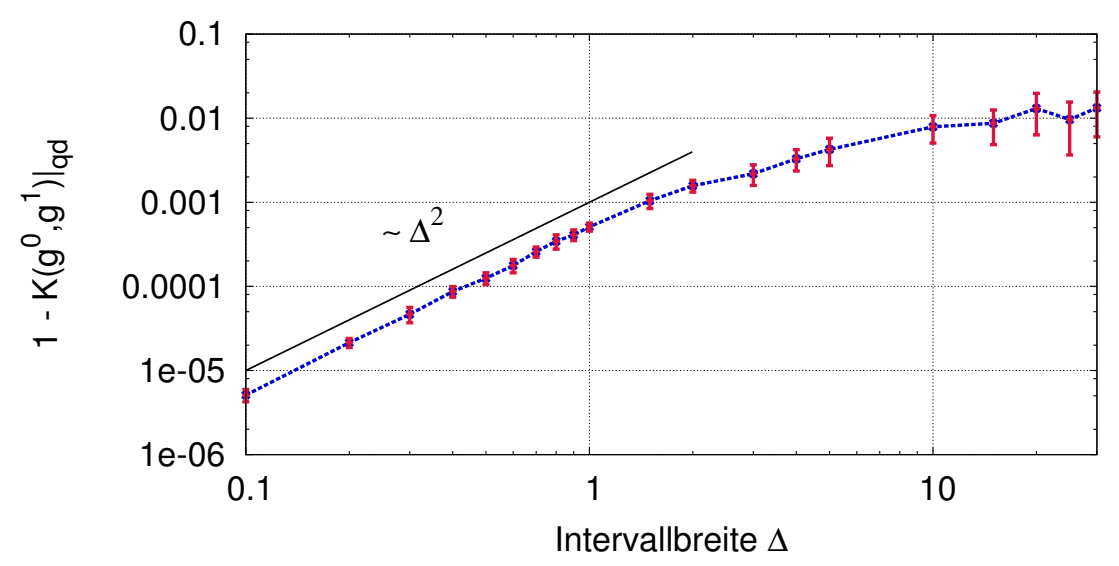

Abbildung 2.6.: Maß für die Unordnung: $1-\left.K\left(g^{0}, g^{1}\right)\right|_{\text {qd }}$ für verschiedene Intervallbreiten $\Delta$ für $N_{Y}=30$ Ketten und $r_{\max }=20$ Runs

lation verwenden wir für die Grafting-Positionen die Abkürzungen $\left.g_{i}\right|_{\mathcal{R} G r=0}=: g_{i}^{0}$, $\left.g_{i}\right|_{\mathcal{R} G r=1}=: g_{i}^{1}$. Es gibt demnach $N_{Y}$ Wertepaare $\left(g_{i}^{0}, g_{i}^{1}\right) ; i=1,2, \ldots, N_{Y}$, die 
zur Berechnung des Korrelationskoeffizienten $K\left(g^{0}, g^{1}\right)$ herangezogen werden. Mit den Mittelwerten

$$
\bar{g}^{0}:=\frac{1}{N_{Y}} \sum_{i=1}^{N_{Y}} g_{i}^{0}=\frac{N_{Y}}{2} \quad, \quad \bar{g}^{1}:=\frac{1}{N_{Y}} \sum_{i=1}^{N_{Y}} g_{i}^{1}
$$

gilt 22,73

$$
K\left(g^{0}, g^{1}\right):=\frac{\sum_{i=1}^{N_{Y}}\left(g_{i}^{0}-\frac{N_{Y}}{2}\right)\left(g_{i}^{1}-\bar{g}^{1}\right)}{\sqrt{\sum_{i=1}^{N_{Y}}\left(g_{i}^{0}-\frac{N_{Y}}{2}\right)^{2} \sum_{i=1}^{N_{Y}}\left(g_{i}^{1}-\bar{g}^{1}\right)^{2}}} .
$$

Analog zum ersten Ordnungsparameter (2.32) sind auch hier zum Erreichen einer besseren Statistik mehrere Runs mit voneinander unabhängigen Zufallszahlen notwendig. Wir schreiben für die Mittelung über $r_{\max }=20$ Simulationsläufe

$$
\left.K\left(g^{0}, g^{1}\right)\right|_{\mathrm{qd}}:=\frac{1}{r_{\max }} \cdot \sum_{r=1}^{r_{\max }} K_{r}\left(g^{0}, g^{1}\right)
$$

Zur besseren Veranschaulichung ist in Abbildung (2.6) der Quenched Disorder-Mittelwert $\left(1-\left.K\left(g^{0}, g^{1}\right)\right|_{\text {qd }}\right)$, der gewissermaßen die Unordnung der Grafting-Konfiguration angibt, als Funktion von unterschiedlichen Intervallbreiten $\Delta$ aufgetragen. Die Fehlerbalken geben die Standardabweichung bezüglich der $r_{\max }$ unabhängigen Runs an, aus denen der Quenched Disorder-Mittelwert gebildet wurde. Man stellt für kleine Intervallbreiten eine stark anwachsende, quadratisch mit $\Delta$ ansteigende Unordnung fest. Dieser Zuwachs an Unordnung wird allerdings bei etwa $\Delta \approx 1$ erwartungsgemäß wegen des mittleren Grafting-Abstandes von $\left\langle d_{\mathrm{Gr}}\right\rangle=1$ mit steigender Intervallbreite abnehmen.

\subsection{Allgemeine Informationen zur Monte-Carlo-Simulationsmethode}

Die Absicht dieses Kapitels besteht in der Beschreibung der Monte-Carlo-Simulationsmethode, die wir verwenden, um das Polymer Brush-Modell nach Abbildung (2.4) $\mathrm{zu}$ simulieren. Zuerst geben wir einige allgemeine Hintergrundinformationen über die Monte-Carlo-Methode an. Anschließend, in Kapitel 2.11, beschreiben wir detailliert die Implementierung für das Polymer Brush-Modell.

Zur Lösung von Problemen und Aufgaben in der statistischen Physik sind computergestützte numerische Methoden [34 91] von sehr großer Bedeutung und mitunter unerlässlich. Insbesondere Systeme mit vielen Freiheitsgraden wie in der Vielteilchenphysik können auf analytischem Wege nicht exakt gelöst werden bzw. würden eine extrem hohe Berechnungszeit in Anspruch nehmen, so dass numerische Lösungsansätze zur Bestimmung makroskopischer Größen notwendig werden. 
Unter den rechnergestützten numerischen Berechnungsmethoden spielt die Anwendung von Monte-Carlo-Simulationen eine bedeutende Rolle [34, 91, 111]. In unserem Fall behandeln wir ein klassisches Vielteilchenproblem im thermischen Gleichgewicht und verwenden als Basis ein Simulationsprogramm mit implementierten Monte-CarloProzeduren von Richard L. C. Vink [111, 112]. Im Folgenden wollen wir in diesem Kontext auf essentielle Schritte und Methoden der Monte-Carlo-Simulationstechnik eingehen.

Wir betrachten ein statistisches System mit konstanter Teilchenzahl $N$ und einem konstanten Volumen $V$ (da sowohl die Kettenlänge $L$ als auch die Breite der GraftingOberfläche $N_{Y}$ konstant gehalten werden), das sich im thermischen Austausch mit einem Wärmebad mit Temperatur $T$ befindet. Somit liegt in unserem Fall ein kanonisches System vor und wir erhalten aus dem Hamiltonian $\mathcal{H}$ die kanonische Zustandssumme des Systems 101

$$
\mathcal{Z}=\operatorname{Sp}\left(\mathrm{e}^{-\beta \mathcal{H}}\right)=\sum_{\mu} \mathrm{e}^{-\beta E_{\mu}}, \text { mit } \beta=\left(k_{B} T\right)^{-1} .
$$

Die Zustandssumme $\mathcal{Z}$ ist von fundamentaler Bedeutung, da sie die gesamte Information eines statistischen Systems beinhaltet und alle thermodynamischen Systemgrößen aus ihr abgeleitet werden können 101.

Die folgende Darstellung der in diesem Kapitel 2.10 gegebenen allgemeinen Informationen zur Monte-Carlo-Simulationsmethode folgt [91, 111.

Jeder Zustand des Systems $\mu$ ist mit einer Energie $E_{\mu}$ und einer Besetzungswahrscheinlichkeit $p_{\mu}$ verknüpft und das Energiespektrum kann sowohl diskret als auch kontinuierlich sein [101. Wir sind an der Bestimmung von makroskopischen Größen $Q$ eines thermischen Systems interessiert. Genauer gesagt soll thermisches Gleichgewicht vorliegen, so dass alle Besetzungswahrscheinlichkeiten zeitunabhängig sind. Mit Hilfe der Monte-Carlo-Methode werden die thermischen Fluktuationen eines Systems simuliert, das heißt im Laufe der Simulationszeit durchläuft das System eine Vielzahl an Zuständen. Zur Berechnung der Erwartungswerte physikalischer Größen wird eine zeitliche Mittelung über alle Zustände vorgenommen [91.

Für ein System, das mit einem Wärmereservoir der Temperatur $T$ in Kontakt steht und sich im thermischen Gleichgewicht befindet, wird nach J. W. Gibbs und L. Boltzmann die Wahrscheinlichkeit, dass sich das System im Zustand mit der Energie $E_{\mu}$ befindet, durch die Besetzungswahrscheinlichkeit wiedergegeben [91,101

$$
p_{\mu}=\frac{1}{\mathcal{Z}} \mathrm{e}^{-E_{\mu} / k_{B} T}
$$

mit der Zustandssumme als Normierungsfaktor.

Die Wahrscheinlichkeitsverteilung in Gl. 2.37) wird Boltzmann-Verteilung genannt. Damit kann für eine interessierende Größe $Q$ eines Systems im thermischen Gleichge- 
wicht der Erwartungswert ermittelt werden 91,111 :

$$
\langle Q\rangle=\frac{1}{\mathcal{Z}} \sum_{\mu} Q_{\mu} \mathrm{e}^{-\beta E_{\mu}}
$$

Zur Verdeutlichung der Vielzahl an Zuständen, die das System einnehmen kann, gehen wir auf die Freiheitsgrade des Modells ein. Jedes bewegliche Segment kann theoretisch jeden Wert auf dem Intervall $\mathcal{G}=\left[0, N_{Y}\right)$ annehmen. Aufgrund der kontinuierlichen Positionswerte der Segmente sind die Spektren der Biege- und Crosslink-Energien ebenfalls kontinuierlich. Hinzu kommen die $\left(\begin{array}{c}N_{\text {Seg }} \\ R\end{array}\right)$ Möglichkeiten, die $R$ Crosslinks auf die

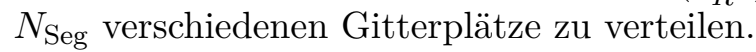

An diesem Beispiel erkennt man, dass im Allgemeinen schon relativ kleine Systeme über eine derart extrem hohe Zahl an Zuständen verfügen, so dass diese bei numerischen Berechnungen innerhalb der normalerweise zur Verfügung stehenden Simulationszeiten bei Weitem nicht alle berücksichtigt werden können.

\section{Importance Sampling}

So kann zum Beispiel die Berechnung der Erwartungswerte $\langle Q\rangle$ von Observablen $Q$ unter Miteinbeziehung aller Zustände, wie in Gleichung (2.38) angegeben, nur für kleine Systeme realisiert werden. Bei größeren Systemen ist dieser Vorgang nicht effizient und es ist sinnvoll, anstelle des exakten Wertes $\langle Q\rangle$ einen guten Schätzwert $Q_{M}$ für $Q \mathrm{zu}$ berechnen, der gegeben ist durch 91,111

$$
Q_{M}=\frac{\sum_{i=1}^{M} Q_{\mu_{i}} p_{\mu_{i}}^{-1} \mathrm{e}^{-\beta E_{\mu_{i}}}}{\sum_{i=1}^{M} p_{\mu_{i}}^{-1} \mathrm{e}^{-\beta E_{\mu_{i}}}},
$$

mit der Eigenschaft, dass im Grenzfall $M \rightarrow \infty$ die Schätzung mit dem exakten Erwartungswert übereinstimmt: $Q_{M}=\langle Q\rangle$ 91,111. In dessen Berechnung gehen jedoch nicht alle dem System zugänglichen Zustände ein, denn während einer Simulationsmessung nimmt das System bei einer Anzahl von $M$ MC-Schritten nur eine winzige Teilmenge $\left\{\mu_{1}, \ldots, \mu_{M}\right\}$ aus der Gesamtheit aller dem System zugänglichen Zustände ein. Zur Verbesserung der Effizienz der Monte-Carlo-Methode ist es aus diesem Grunde plausibel, nur diejenigen Zustände zu berücksichtigen, die wesentlich zur Messung beitragen. Diese Methode wird Importance Sampling genannt [91,111].

Anstatt allen Zuständen die gleiche Auswahlwahrscheinlichkeit $p_{\mu}$ zuzuordnen, erweist es sich als optimal, die $M$ Zustände gemäß der Boltzmann-Statistik $p_{\mu} \propto \exp \left(-\beta E_{\mu}\right)$ auszuwählen. Damit ergibt sich für den Schätzer in $(2.39)$ der folgende Ausdruck 91, 111 .

$$
Q_{M}=\frac{1}{M} \sum_{i=1}^{M} Q_{\mu_{i}}
$$

das heißt die Boltzmann-Faktoren heben sich auf und Importance Sampling ist bei allen Temperaturen $T$ anwendbar.

Der effizienten Generierung einer Menge an Zuständen, die der Boltzmann-Statistik genügen, liegt ein Markov-Prozess zugrunde. Bei diesem Mechanismus geht man von 
einem initialen Zustand $\mu$ aus und generiert von diesem ausgehend einen neuen Zustand $\nu$. Die damit verbundene Übergangswahrscheinlichkeit, dass das System vom Zustand $\mu$ in den Zustand $\nu$ übergeht, wird mit $T(\mu \rightarrow \nu)$ bezeichnet. Allgemein gilt für die Übergangswahrscheinlichkeiten die Normierungsbedingung

$$
\sum_{\nu} T(\mu \rightarrow \nu)=1
$$

Die wiederholte Anwendung des Markov-Prozesses führt zur Generierung einer Markovkette, das heißt ausgehend vom Zustand $\mu$ wird ein neuer Zustand erzeugt, der wiederum beim nächsten Markov-Prozess als Ausgangspunkt für die Erzeugung des nächsten Zustands dient, wobei dieser Prozess in der Weise fortgesetzt wird. Damit wird im Einklang mit der Boltzmann-Verteilung eine Folge von Zuständen generiert, und das System nimmt den Gleichgewichtszustand ein 91, 111.

Zum Erreichen des Ziels der Erzeugung von Zuständen, die der Boltzmann-Statistik genügen, sind neben einer hinreichend langen Simulationszeit zur thermischen Equilibrierung des Systems außerdem zwei Punkte notwendig, die im Folgenden erläutert werden: Die Ergodizität und die sogenannte Detailed Balance.

\section{Ergodizität}

Eine notwendige, aber nicht hinreichende Bedingung stellt die Ergodizität des Simulationsalgorithmus dar. Das bedeutet, dass jeder mögliche Zustand des Systems im Rahmen des Markov-Prozesses von jedem beliebigen Zustand aus erreichbar sein muss. Die Anzahl der dafür benötigten MC-Schritte hat endlich zu sein 91,111.

\section{Detailed Balance}

Dass die Markov-Kette im thermischen Gleichgewicht Zustände generiert, die die gewünschte Boltzmann-Verteilung aufweisen, wird durch die Detailed Balance-Bedingung garantiert:

$$
p_{\mu} T(\mu \rightarrow \nu)=p_{\nu} T(\nu \rightarrow \mu) .
$$

Um im Speziellen zu erreichen, dass der Markov-Prozess im thermischen Gleichgewicht boltzmannverteilte Zustände erzeugt, muss für die Übergangswahrscheinlichkeiten in Gleichung (2.42) die Bedingung

$$
\frac{T(\mu \rightarrow \nu)}{T(\nu \rightarrow \mu)}=\frac{p_{\nu}}{p_{\mu}}=\mathrm{e}^{-\beta\left(E_{\nu}-E_{\mu}\right)}
$$

gelten 91,111 .

Mit den positiven Wahrscheinlichkeiten $p_{\mu}$ und $p_{\nu}$ und einer ebenso von Null verschiedenen Übergangswahrscheinlichkeit $T(\mu \rightarrow \nu)$ folgt daraus die Reversibilität eines durch einen MC-Schritt generierten Überganges zwischen zwei Zuständen $(\mu \leftrightarrow \nu)$. Diese Eigenschaft muss notwendigerweise für ein Monte-Carlo-Schema gewährleistet sein. Wir verwenden im Programm als Monte-Carlo-Methode den Metropolis-Algorithmus, der unter anderem von N. Metropolis eingeführt wurde [88, 91, 111]. Hierbei werden, wie 
oben diskutiert, über einen Markov-Prozess in einer effizienten Weise Zustände gemäß der Boltzmann-Statistik generiert.

Der Metropolis-Algorithmus verläuft wie im folgenden Schema dargestellt [91, 111]:

1. ausgehend vom Zustand $\mu$ wird per Zufallsbewegung (Random-Move) ein neuer Zustand $\nu$ vorgeschlagen.

2. dieser neue Zustand $\nu$ wird mit einer durch die entsprechende Akzeptanzrate festgelegten Wahrscheinlichkeit akzeptiert oder abgelehnt.

3. bei Akzeptanz wird der neue Zustand $\nu$ angenommen, andernfalls bleibt das System im Zustand $\mu$. Dieser Prozess wird mehrfach wiederholt.

Dabei ist wichtig zu betonen, dass die durch den Algorithmus erzeugten Zustandsänderungen in der Regel klein sind.

Befindet sich das System im thermischen Gleichgewicht, so sind thermische Fluktuationen in Relation zur Gesamtenergie des Systems relativ klein. Das System nimmt fast ausschließlich nur Zustände an, bei denen die dazugehörigen Gesamtenergien nicht weit auseinanderliegen, so dass die Zustandsänderungen nur zu kleinen Energieänderungen führen 91 .

Die Übergangswahrscheinlichkeit lässt sich als ein Produkt zweier Wahrscheinlichkeiten angeben [91, 111]:

$$
T(\mu \rightarrow \nu)=s(\mu \rightarrow \nu) \cdot a(\mu \rightarrow \nu) .
$$

Mit $s(\mu \rightarrow \nu)$ wird die Wahrscheinlichkeit bezeichnet, dass der Algorithmus ausgehend vom Zustand $\mu$ den neuen Zustand $\nu$ auswählt, während die Akzeptanzrate $a(\mu \rightarrow \nu)$ die Wahrscheinlichkeit angibt, mit der der vorgeschlagene Zustand $\nu$ akzeptiert wird.

Mit (2.44) kann die Detailed Balance-Bedingung (2.43) mit Hilfe der Akzeptanzraten geschrieben werden als 91,111

$$
\frac{a(\mu \rightarrow \nu)}{a(\nu \rightarrow \mu)}=\frac{s(\nu \rightarrow \mu)}{s(\mu \rightarrow \nu)} \mathrm{e}^{-\beta\left(E_{\nu}-E_{\mu}\right)} .
$$

Die Schrittbreite $f$, innerhalb der ein neuer Zustand per Bend-Move vorgeschlagen wird, ist für alle beweglichen Segmente gleich. Da auch die Zahl der Möglichkeiten für Crosslink-Moves während einer Messung konstant bleibt, folgt daraus eine konstante Auswahlwahrscheinlichkeit der Zustände. Somit gilt $s(\mu \rightarrow \nu)=s(\nu \rightarrow \mu)$, das heißt deren Quotient hebt sich auf und 2.45 vereinfacht sich zu

$$
\frac{a(\mu \rightarrow \nu)}{a(\nu \rightarrow \mu)}=\mathrm{e}^{-\beta\left(E_{\nu}-E_{\mu}\right)}
$$

Im Zusammenhang mit der Wahl der optimalen Akzeptanzrate $a(\mu \rightarrow \mu)$ muss in der Detailed Balance-Bedingung (2.46) berücksichtigt werden, dass $a(\mu \rightarrow \nu)$ als Wahrscheinlichkeitsmaß lediglich Werte zwischen Null und Eins annehmen kann. Haben wir einen Übergang vom Zustand $\mu$ in den Zustand $\nu$ mit den Energien $E_{\nu}>E_{\mu}$, so gilt gemäß 2.45) für die beiden Akzeptanzraten $a(\nu \rightarrow \mu)>a(\mu \rightarrow \nu)$. Dabei wird die größere Akzeptanzrate gleich Eins gesetzt $(a(\nu \rightarrow \mu)=1)$, und das Kriterium des 
Metropolis-Algorithmus lautet 91, 111

$$
a(\mu \rightarrow \nu)=\left\{\begin{array}{ll}
\mathrm{e}^{-\beta\left(E_{\nu}-E_{\mu}\right)} & , \text { falls } E_{\nu}-E_{\mu}>0 \\
1 & , \text { sonst }
\end{array} .\right.
$$

Dies bedeutet, dass ein Übergang zu einem Zustand niedrigerer Energie stets beibehalten wird, während ein energetisch höher gelegener Zustand nur mit einer Wahrscheinlichkeit entsprechend (2.47) akzeptiert wird.

\subsection{Anwendung auf das Polymer Brush-Modell}

Ausgangspunkt für die numerische Berechnung ist die Gesamtenergie des Modells. Diese setzt sich aus der gesamten Biegeenergie $\mathcal{H}_{\kappa}$ der $N_{Y}$ modellierten Polymerketten (2.18) und der Energie der im Modell eingebrachten $R$ Crosslinks $\mathcal{H}_{\text {xlink }}$ zusammen (2.21):

$$
\mathcal{H}_{\text {Ges }}=\mathcal{H}_{\kappa}+\mathcal{H}_{\text {xlink }} .
$$

Betrachten wir ausschließlich die Polymerketten ohne eingebrachte Crosslinks, so gibt es einen eindeutigen Grundzustand (GS), der die Energie minimiert. Dieser Zustand wird erreicht, wenn alle Ketten parallel zur $x$-Achse ausgerichtet sind, das heißt $u_{s, i}=0$ $\forall i=0,1, \ldots, N_{Y} ; s=0,1, \ldots, L$. Alle Segmente befinden sich in der Ruhelage, erfahren demzufolge keine Auslenkung und die Biegeenergie ist Null: $\mathcal{H}_{\kappa}^{\mathrm{GS}}=0$. Aufgrund der Tatsache, dass alle Bond-Vektoren $\boldsymbol{t}_{s, i}$ in dieselbe Richtung zeigen, ist der Grundzustand auch ein geordneter Zustand [11]. Alle anderen davon abweichenden Kettenkonformationen sind mit Kettenkrümmungen und damit mit einem Zuwachs an Biegeenergie verbunden, das heißt, alle übrigen möglichen Zustände sind ungeordnete, angeregte Zustände mit $\mathcal{H}_{\kappa} \geq \mathcal{H}_{\kappa}^{\mathrm{GS}}$.

Die Energieänderungen im hier betrachteten Modellsystem werden durch Zustandsänderungen $(\mu \rightarrow \nu)$ generiert. Dabei wird zunächst via Monte-Carlo-Schritt entschieden, ob die Änderung durch einen Random-Move eines Crosslinks oder durch einen BendMove eines Segments verursacht wird. Beim Random-Move wird per Zufall einer der $R$ Crosslinks ausgewählt und wiederum per Zufallsschritt einem bislang leeren Gitterplatz zugewiesen, während beim Bend-Move ein zufällig ausgewähltes Segment innerhalb einer festgelegten Breite um die ursprüngliche Position per Zufall ausgelenkt wird.

\section{Der Aufbau des Simulationsprogramms}

Im Folgenden sind die wesentlichen Schritte des Programmablaufs wiedergegeben:

- beim Start des Programms werden die in Kapitel 2.8 aufgelisteten Parameter eingelesen

- die Positionen der Grafting-Points auf der Substratoberfläche werden festgelegt: je nach Parameterwahl sind diese äquidistant $(\mathcal{R} G r=0)$ oder zufällig verteilt $(\mathcal{R} G r=1)$ 
- es wird die Start-Konfiguration der Kettensegmente zu Beginn der Simulation vorgegeben, das heißt entweder mit zufälliger Auslenkung $u_{s, i} \operatorname{der}(L-1) \cdot N_{Y}$ beweglichen Kettensegmente oder ohne Auslenkung, das heißt aus der Ruhelage

- es werden $R$ Crosslinks gemäß den in Kapitel 2.7 aufgeführten Regeln zufällig auf die $N_{\text {Seg }}$ Segmente verteilt

Vor dem Start der Dauerschleife als wesentlichen Simulationsteil, in welchem per Metropolis-Algorithmus die Zustandsänderungen erzeugt werden, sind die oben genannten Schritte zur Festlegung der Grafting-, Segment-, und Crosslink-Positionen für den Simulationsablauf unabdingbar. Denn zu Beginn muss ein wohldefinierter Zustand vorliegen, der die festgelegten Werte (Parameter, Grafting-Point-Positionen, Anfangskonfiguration der Ketten und Gitterplatzbelegung der $R$ Crosslinks) vorgibt. Anschließend startet die eigentliche MC-Simulation nach folgendem Schema:

Eine Timing-Routine legt eine Zahl an Monte-Carlo-Schritten fest, die in einem SweepZyklus verwendet wird 91, 111. Innerhalb dieser Programmsequenz wird per MCSchritt zwischen der Auslenkung eines Segments (Bend-Move) und der Versetzung eines Crosslinks (Crosslink-Move) entschieden. Das Verhältnis dieser beiden Wahrscheinlichkeiten für die Segmentauslenkung $p_{\mathrm{b}}$ und die Crosslinkversetzung $p_{\mathrm{xl}}$ ist ein weiterer, beliebig wählbarer Parameter und beträgt in unserer Simulation $p_{\mathrm{b}}: p_{\mathrm{xl}}=9: 1$.

\section{Bend-Move}

Fällt die Wahl auf einen Bend-Move, so wird per Monte-Carlo-Schritt eines der $(L-1) \cdot N_{Y}$ beweglichen Segmente $(s, i) ; s=2,3, \ldots, L ; i=1,2, \ldots, N_{Y}$ ausgewählt. Anschließend führt das Programm ausgehend von der alten Position des betreffenden Segments $\boldsymbol{r}_{s, i}^{\mu}$ einen weiteren Monte-Carlo-Schritt aus, um eine zufällig erzeugte Auslenkung aus der alten Position hin zu einer neuen Position $\boldsymbol{r}_{s, i}^{\nu}$ vorzuschlagen:

$$
\boldsymbol{r}_{s, i}^{\mu}=\left(b \cdot s, u_{s, i}^{\mu}\right) \underset{\mathrm{MC}-\mathrm{Schritt}}{\longrightarrow} \boldsymbol{r}_{s, i}^{\nu}=\left(b \cdot s, u_{s, i}^{\mu}+(0.5-\xi) \cdot f\right)=:\left(b \cdot s, u_{s, i}^{\nu}\right),
$$

mit der bereits eingeführten gleichverteilten Zufallszahl $\xi \in[0,1)$ und einer festgelegten Intervallbreite von $f=3 / 10$, die die in einem MC-Schritt maximal mögliche Auslenkungsbreite angibt. Wie in Kapitel 2.5 bereits erklärt wurde, zeichnet sich das System offensichtlich nicht durch eine feste, diskrete Zustandszahl, sondern durch ein kontinuierliches Energiespektrum aus.

Wie man sich leicht veranschaulichen kann, ist das Kriterium der Ergodizität erfüllt. Denn jedes Segment hat theoretisch die Möglichkeit, durch Fluktuationen jeden Wert auf dem Intervall $\mathcal{G}$ innerhalb einer endlichen Zahl an MC-Schritten zu erreichen, das heißt $u_{s, i} \in\left[0, N_{Y}\right) \forall s=2,3, \ldots, L ; i=1,2, \ldots, N_{Y}$.

Dies gilt auch für den Fall, dass zwischen den Ketten eine Excluded Volume-Wechselwirkung wirkt und die Ketten sich nicht durchdringen oder kreuzen können. Allerdings besteht hinsichtlich der Gesamtheit aller möglichen Zustände die Einschränkung, dass die Segmente in einer wohlgeordneten Abfolge auf dem Intervall $\mathcal{G}$ angeordnet sein müssen:

$$
u_{s, 1} \leq u_{s, 2} \leq \ldots \leq u_{s, N_{Y}}+\text { zykl. } \in\left[0, N_{Y}\right) \quad \forall s=2,3, \ldots, L
$$


(für $s \in\{0,1\}$ ist die Forderung (2.50) trivialerweise erfüllt).

Für die Akzeptanzrate $a(\mu \rightarrow \nu)$ ist die Energiedifferenz $\triangle E=E_{\nu}-E_{\mu}$, die das Gesamtsystem durch den vorgeschlagenen MC-Schritt erfährt, entscheidend. Da das gewählte Segment mit bis zu zwei Crosslinks verbunden sein kann, geht in $\triangle E$ neben der Änderung der Biegeenergie gegebenenfalls auch eine Änderung der Crosslinkenergie ein. Zur effizienten und schnelleren Berechnung berechnet das Programm nach jedem Monte-Carlo-Schritt lediglich die lokale Energieänderung in der Umgebung des betreffenden Segments, da alle restlichen Ketten- und Crosslink-Koordinaten gleich bleiben und damit keine Energieänderung erfahren.

Die mit dem Bend-Move einhergehende Energiedifferenz $\triangle E$ wird unter Verwendung der in Gleichung (2.48) definierten Gesamtenergie des Modells berechnet.

Bei einem vorgeschlagenen Bend-Move besteht die Möglichkeit, dass das betreffende Segment ein benachbartes Segment überspringt, so dass sich durch diesen Schritt die Ketten überlappen oder kreuzen würden. Bei vorhandener Excluded Volume-Wechselwirkung $(E x-V o l=1)$ wird dieser Schritt durch den Algorithmus abgelehnt.
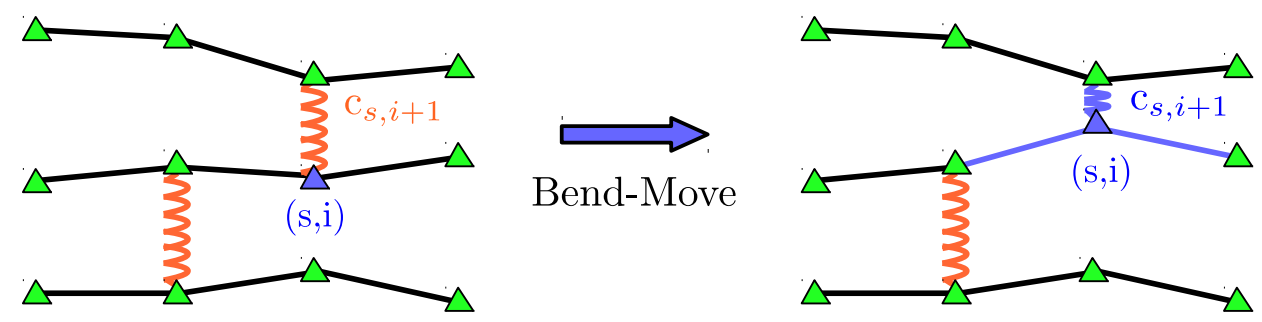

Abbildung 2.7.: Schematische Darstellung eines Bend-Moves. Das Segment $(s, i)$ wird nach oben ausgelenkt, wodurch sich die Länge des Crosslinks $c_{s, i+1}$ verringert.

Abbildung 2.7) zeigt schematisch die Durchführung eines Bend-Moves anhand eines beliebigen Segments mit den Koordinaten $(s, i)$. Im gezeigten Beispiel wird das Segment nach oben ausgelenkt. Dabei wird an der Stelle zum Einen die lokale Biegeenergie verändert, zum Anderen wird die Länge $d_{s, i+1}$ des Crosslinks $c_{s, i+1}$ verringert und damit auch seine Energie.

\section{Crosslink-Move}

Bei der Auswahl eines Crosslink-Moves wird per Zufall ein mit einem Crosslink besetzter Gitterplatz $\left(s_{1}, i_{1}\right)$ gewählt. Die im Metropolis-Algorithmus vorgeschlagene Zustandsänderung besteht in der Versetzung dieses Crosslinks auf einen zufällig gewählten neuen, bisher leeren Gitterplatz $\left(s_{2}, i_{2}\right)$. Die damit verbundene Energiedifferenz $\triangle E_{\mathrm{xl}}$ wird entsprechend Gleichung (2.48) berechnet.

Auch hier gilt, dass der vorgeschlagene Crosslink-Move auf jeden Fall akzeptiert wird, falls $\triangle E_{\mathrm{xl}}<0$ gilt, während bei Energieerhöhung $\triangle E_{\mathrm{xl}}>0$ dieser lediglich mit einer Wahrscheinlichkeit von $a(\mu \rightarrow \nu)=\mathrm{e}^{-\beta \triangle E_{\mathrm{xl}}}$ angenommen wird. 

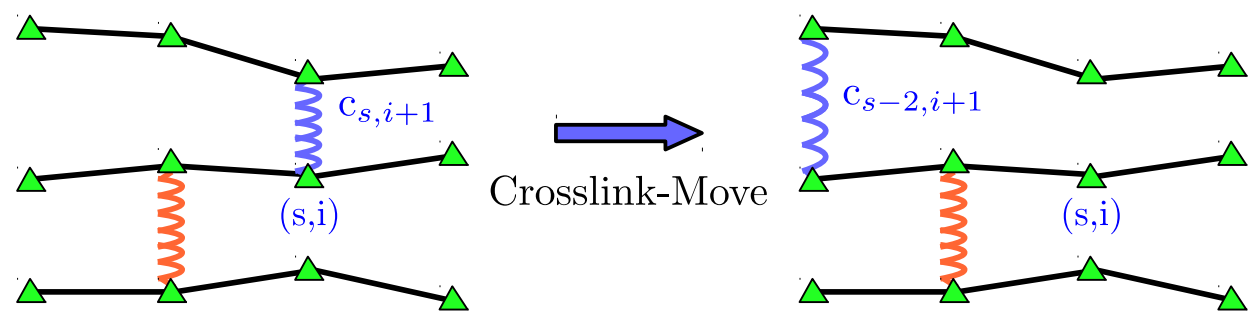

Abbildung 2.8.: Schematische Darstellung eines Crosslink-Moves. Der Crosslink $c_{s, i+1}$ wird auf den Gitterplatz $c_{s-2, i+1}$ versetzt.

Zur einfacheren Beschreibung zeigt Abbildung (2.8) das Schema eines Crosslink-Moves. Im beispielhaft aufgeführten Fall wird der Crosslink $c_{s, i+1}$ ausgewählt und auf einen anderen, bislang leeren Gitterplatz $(s-2, i+1)$ versetzt. Mit der Einnahme eines neuen Gitterplatzes ist für den umplatzierten Crosslink in der Regel auch eine neue Crosslink-Länge verbunden.

\section{Der Sweep-Zyklus in Endlosschleife}

Nach dem Durchlauf des Sweep-Zyklus und der damit verbundenen Ausführung einer großen Zahl an Bend-Moves $\left(p_{\mathrm{b}}=9 / 10\right)$ und Crosslink-Moves $\left(p_{\mathrm{xl}}=1 / 10\right)$ werden verschiedene Datensätze zur Ermittlung verschiedener physikalischen Größen herausgeschrieben (siehe folgende Kapitel 2.13 bis 2.23). Dies betrifft sowohl instantane Werte (wie zum Beispiel die momentanen Segmentpositionen) als auch die fortlaufende Mittelung zur Bestimmung des zeitlichen Mittelwertes einer Größe wie zum Beispiel die mittlere Gesamtenergie.

Innerhalb einer Endlosschleife, die als Herzstück des Simulationsprogramms angesehen werden kann, wird der Sweep-Zyklus erneut durchlaufen und es werden solange Daten herausgeschrieben, bis das Programm beendet oder abgebrochen wird. Der Programmabbruch geschieht in der Regel, wenn das Modellsystem nach Erreichen des Gleichgewichtszustandes genügend Zyklen bzw. MC-Schritte durchlaufen hat, so dass den gewünschten und zu bestimmenden physikalischen Größen eine hinreichend gute Statistik zugrunde liegt.

Zur übersichtlicheren Veranschaulichung sind in Abbildung (2.9) die wesentlichen Abläufe des Simulationsprogramms abgebildet. 
- Einlesen der Parameter

- Festlegung der Grafting-Positionen, Ketten-Konfiguration und Crosslink-Verteilung

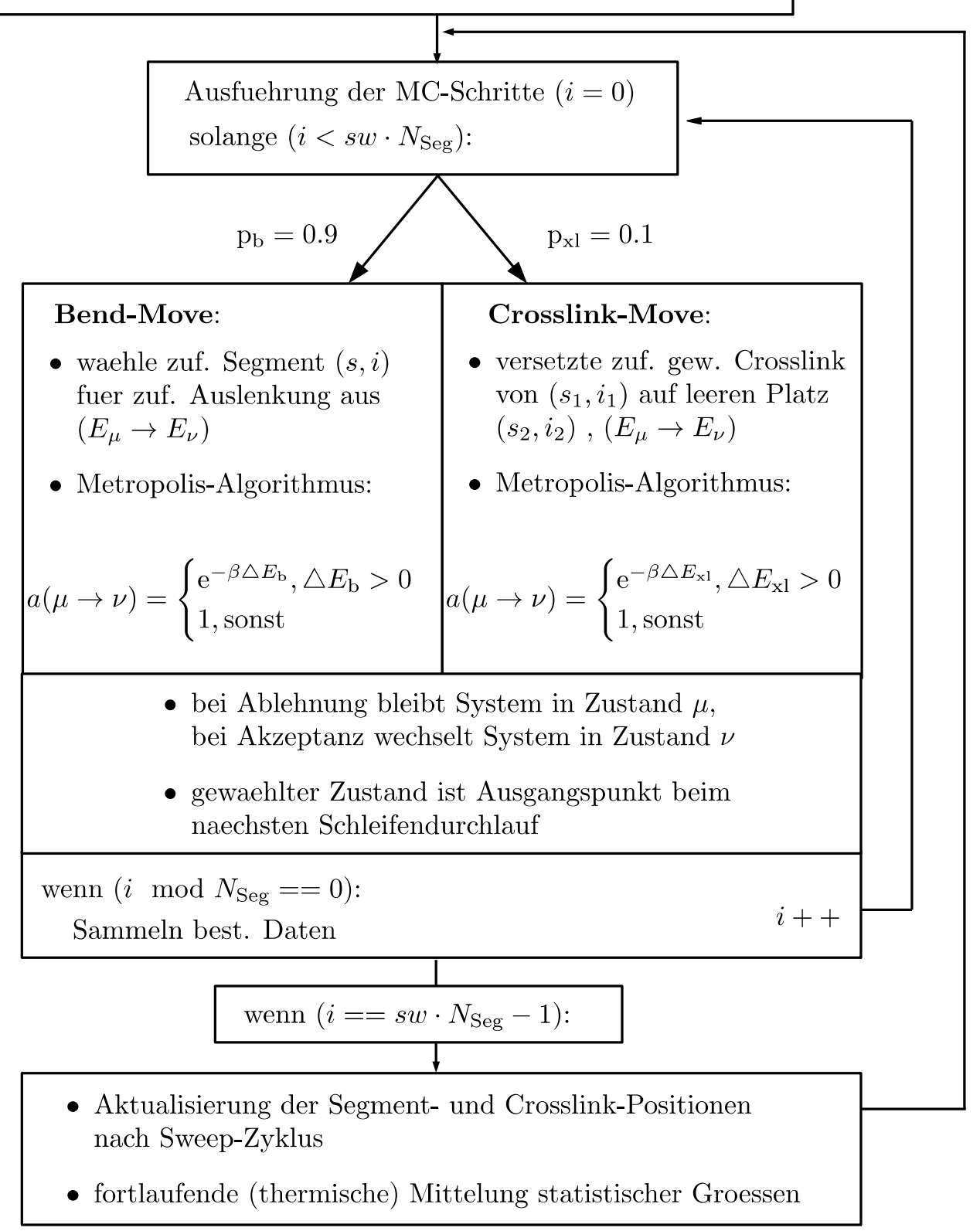

Abbildung 2.9.: Diagrammatische Darstellung des Algorithmus des Simulationsprogramms 


\subsection{Simulationsergebisse}

In diesem Kapitel stellen wir die Ergebnisse aus den Monte-Carlo-Simulationen des Polymer Brush-Modells vor. Zuerst diskutieren wir die mit Bezug auf die Simulationsdauer notwendigen Begrenzungen der Systemgröße. Danach werden strukturelle Eigenschaften des Polymer Brush-Modells aufgezeigt, die mit Hilfe des statischen Strukturfaktors analysiert werden können. Hinzu kommen weitere interessante Systemgrößen wie Fluktuationen und Richtungskorrelationen der Segmente sowie Winkelverteilungen der Ketten. Zu guter Letzt beschreiben wir die Existenz des Memory-Effektes, der aufzeigt, dass die selbe Grafting-Konfiguration stets in die selbe persistente Polymerstruktur mündet.

\subsection{Einschränkungen der Systemgröße}

Das zu analysierende System hat im Verlauf eines Simulationsdurchgangs stets die gleiche Teilchenzahl $N_{\text {Seg }}$ sowie ein konstantes, zweidimensionales Volumen $V \equiv \mathcal{P} \times \mathcal{G}$ (siehe Kapitel 2.5) und steht im Kontakt mit einem Wärmereservoir der Temperatur T. Als kanonisches Ensemble 101 muss das System notwendigerweise im thermischen Gleichgewicht vorliegen, wobei das Erreichen des Gleichgewichtszustandes einen irreversiblen Vorgang darstellt. Die Irreversibiltät zeichnet makroskopische Phänomene wie den Austausch thermischer Energie aus und bei gegebenen Randbedingungen gibt es in der Regel nur genau eine Gleichgewichtskonfiguration, der das System entgegenstrebt. Dieser Gleichgewichtszustand wird, sofern sich an den äußeren Bedingungen nichts ändert, dauerhaft beibehalten und es kommt lediglich zur Ausführung von thermischen Fluktuationen um die Gleichgewichtskonfiguration [90].

Befindet sich ein System im thermischen Gleichgewicht, so entsprechen die Besetzungswahrscheinlichkeiten der dem System zugänglichen Zustände der Boltzmann-Verteilung. Unter dem thermodynamischen Limes versteht man in der statistischen Mechanik ein System im Grenzfall unendlich vieler Teilchen $(N \rightarrow \infty)$ und eines unendlichen Volumens $(V \rightarrow \infty)$, wobei die Teilchendichte konstant gehalten wird $\left(\frac{N}{V}=\right.$ konst.) 101 . In diesem Grenzfall haben wir es mit einem makroskopischen System zu tun, das sich mit Hilfe der Gesetze der Thermodynamik beschreiben lässt. Mit wachsender Teilchenzahl $N$ wird die Aussage über eine makroskopische Größe immer schärfer, da deren relative Schwankung, das heißt, das Verhältnis von Schwankung zu Mittelwert, in diesem Grenzfall verschwindet. Die Begründung dafür ist im zentralen Grenzwertsatz zu finden und es zeigt sich, dass die Fluktuationen mit $1 / \sqrt{N}$ skalieren 101 .

Bei numerischen Berechnungen ist man aufgrund technischer Grenzen wie Speicherplatz und Rechenzeit bezüglich der zu simulierenden Systemgröße und den damit verbundenen Freiheitsgraden eingeschränkt. Um bei der computerbasierten Analyse der gegebenen Rechenleistung gerecht zu werden, sind im Allgemeinen nur Systemgrößen möglich, die sich weit entfernt vom thermodynamischen Limes befinden. 
Die Zielstellung besteht in der Wahl einer geeigneten Systemgröße. Bei einem zu großen System mit entsprechend vielen Freiheitsgraden wird das System eine zu lange Equilibierungszeit benötigen. Daher beabsichtigen wir, die Systemgröße in Form von Kettenund Segmentanzahl derart zu wählen, dass sich das System in relativ kurzer Zeit im thermischen Gleichgewicht befindet und dass gleichzeitig eine ausreichende Systemgröße gewährleistet ist.

Um die Simulationszeit abschätzen zu können, die das System benötigt, um in den Gleichgewichtszustand zu kommen, führen wir die „Magnetisierung“ (in Anlehnung an eine Bezeichnung aus dem Magnetismus [101]) $M(t)$ ein:

$$
M(t):=\frac{1}{N_{Y}} \cdot \sum_{i=1}^{N_{Y}}\left\langle u_{L, i}(t)\right\rangle \quad \text { mit } \quad\left\langle u_{s, i}(t)\right\rangle:=\frac{1}{t} \int_{0}^{t} u_{s, i}\left(t^{\prime}\right) \mathrm{d} t^{\prime} .
$$

Die Größe $\left\langle u_{s, i}(t)\right\rangle$ bezeichnet die fortlaufende Mittelung (für die im Grenzfall unendlicher Zeit gilt: $\left.\left\langle u_{s, i}(t)\right\rangle \underset{t \rightarrow \infty}{\longrightarrow}\left\langle u_{s, i}\right\rangle\right)$ und $u_{s, i}\left(t^{\prime}\right)$ die Auslenkung zum Zeitpunkt $t^{\prime}$.

Die „Magnetisierung“ $M(t)$ beschreibt für die Segmente am Kettenende $(s=L)$ die transversale Abweichung aus der Ruhelage, gemittelt über alle $N_{Y}$ Ketten und über die Zeit $t$, die der Anzahl der durchlaufenen Schleifendurchgängen entspricht. Zusätzlich gibt die Varianz [73] der „Magnetisierung" $\sigma_{M}^{2}(t)$ Aufschluss über die Abnahme der Fluktuationen als Funktion der Zeit, so dass darüber das Erreichen des Gleichgewichtszustandes abgeleitet werden kann:

$$
\sigma_{\mathrm{M}}^{2}(t):=\frac{1}{N_{Y}} \cdot \sum_{i=1}^{N_{Y}}\left(\left\langle u_{L, i}(t)\right\rangle\right)^{2}-\left(\frac{1}{N_{Y}} \cdot \sum_{i=1}^{N_{Y}}\left\langle u_{L, i}(t)\right\rangle\right)^{2} .
$$

Mit Hilfe geeigneter Parametervorgaben halten wir das System möglichst einfach, das heißt wir verwenden äquidistant gegraftete Ketten $(\mathcal{R} G r=0)$ ohne Excluded Volu$m e$-Wechselwirkung $(E x-V o l=0)$ und ohne die Hinzunahme von Crosslinks $(\rho=0)$. Das Ziel besteht darin, den qualitativen Einfluss der beiden Systemgrößen-Parameter (Anzahl der Segmente pro Kette $(L+1)$ und Anzahl der Ketten $N_{Y}$ ) auf die Equilibrierungszeit zu messen.

Abbildung (2.10) zeigt für fünf beliebig gewählte Systemgrößen den Verlauf der „Magnetisierung" $M(t)$ aus Einzelmessungen als Funktion der Anzahl an durchlaufenen Schleifen $t$. Darüber hinaus sind in Abbildung (2.11) die dazugehörigen Standardabweichungen der „Magnetisierung“ aufgetragen, gemittelt über $r_{\max }=5$ unabhängige Runs, die wegen $\mathcal{R} G r=0$ mit $\left.\sigma_{M}(t)\right|_{\mathrm{qd}} ^{\mathcal{R} G r=0}$ bezeichnet werden.

Bei einem equilibrierten System erwartet man, dass freie Ketten und damit auch die Kettenenden ohne Vorzugsrichtung Fluktuationsbewegungen ausführen. Die Wahrscheinlichkeit für eine Segmentauslenkung $u_{s, i}$ in positive $y$-Richtung ist dabei genauso groß wie die Auslenkung in die entgegengesetzte Richtung. Folglich ist mit dem Erreichen des thermischen Gleichgewichts verbunden, dass die "Magnetisierung“ in der Langzeitmittelung verschwindet und deren Schwankung bzw. Standardabweichung gemäß des zentralen Grenzwertsatzes der Statistik mit $t^{-1 / 2}$ abnimmmt 73 . Die charakteristischen Merkmale für das Auftreten des Gleichgewichtszustandes sind also $M(t) \underset{t \rightarrow \infty}{\longrightarrow} 0$ und $\left.\sigma_{M}(t)\right|_{\mathrm{qd}} ^{\mathcal{R} G r=0} \propto \frac{\tilde{a}}{\sqrt{t}}$, mit einer Konstanten $\tilde{a}$. 


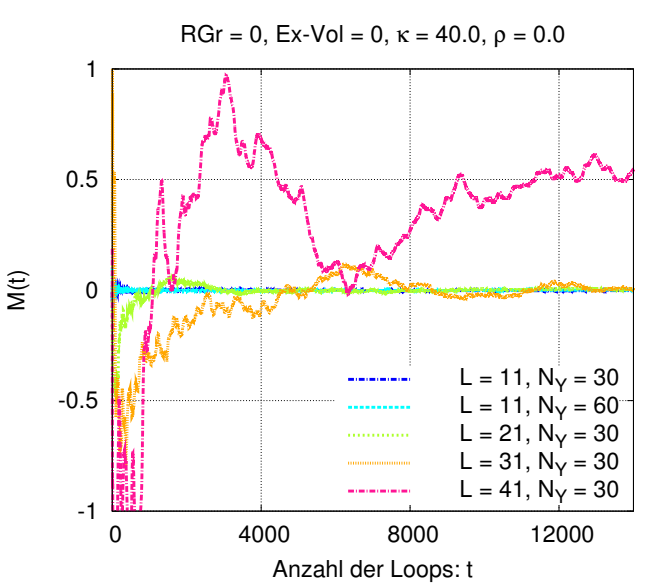

Abbildung 2.10.: „Magnetisierung“" für unterschiedliche Systemgrößen als Funktion von $t$

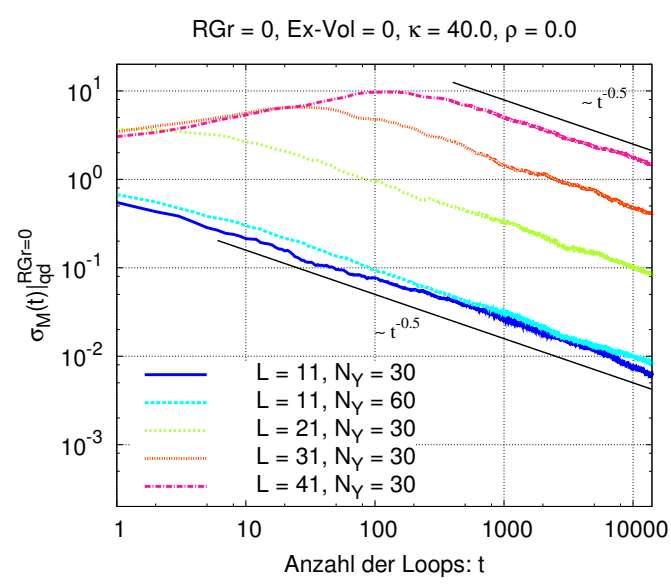

Abbildung 2.11.: Schwankung der „Magnetisierung" $\left.\sigma_{M}(t)\right|_{\mathrm{qd}} ^{\mathcal{R} G r=0}$ für unterschiedliche Systemgrößen als Funktion von $t$

Beide Diagramme machen den Einfluss der Kettenlänge auf die Equilibrierungsdauer deutlich. Je kleiner die Kettenlänge $L$, desto weniger Schleifendurchgänge sind zum Erreichen des Gleichgewichtszustandes notwendig.

Sehen wir uns zum Beispiel explizit das größte System mit $L=41$ an, so lässt sich erkennen, dass auch nach mehr als $1,2 \cdot 10^{4}$ Loops noch keine vollständige Equilibrierung des Systems vorliegt. Nach etwa $10^{3}$ Schleifendurchgängen allerdings nehmen die Schwankungen gemäß des $t^{-1 / 2}$-Gesetztes ab, liegen jedoch betragsmäßig um mehr als zwei Größenordnungen über denen des kleinsten Systems mit $L=11$.

Ebenfalls kann man leicht ablesen, dass im Gegensatz zur Erhöhung der Kettenlänge $L$ die Erhöhung der Kettenanzahl $N_{Y}$ nur einen geringen Einfluss auf die Equilibrierungsdauer hat.

Alternativ können Equilibrierungscharakteristiken auch mit Hilfe der mittleren quadratischen Verschiebung (Mean Square Displacement, (MSD))

$$
\left\langle x_{s, i}(t)\right\rangle:=\frac{1}{t} \int_{0}^{t}\left(u_{s, i}\left(t^{\prime}\right)\right)^{2} \mathrm{~d} t^{\prime}
$$

erläutert werden. Wir betrachten deren Varianz 73], sie ist wie folgt definiert:

$$
\sigma_{\mathrm{MSD}}^{2}(t):=\frac{1}{N_{Y}} \cdot \sum_{i=1}^{N_{Y}}\left(\left\langle x_{L, i}(t)\right\rangle\right)^{2}-\left(\frac{1}{N_{Y}} \cdot \sum_{i=1}^{N_{Y}}\left\langle x_{L, i}(t)\right\rangle\right)^{2} .
$$

In Abbildung (2.12) ist die Mittelung der Standardabweichung der mittleren quadratischen Verschiebung $\left.\sigma_{\mathrm{MSD}}(t)\right|_{\mathrm{qd}} ^{\mathcal{R} G r=0}$ über $r_{\max }=5$ unabhängige Runs für zwei unterschiedliche Kettenlängen und vier unterschiedliche $\kappa$-Werte als Funktion der Schleifendurchgänge $t$ aufgetragen. Das Verhalten ist vergleichbar mit dem der oben besprochenen „Magnetisierung“. Erwartungsgemäß nimmt die Proportionalitätskonstante $\tilde{a}$ mit zunehmender Biegesteifigkeit und damit mit kleiner werdenden Auslenkungen ab. 


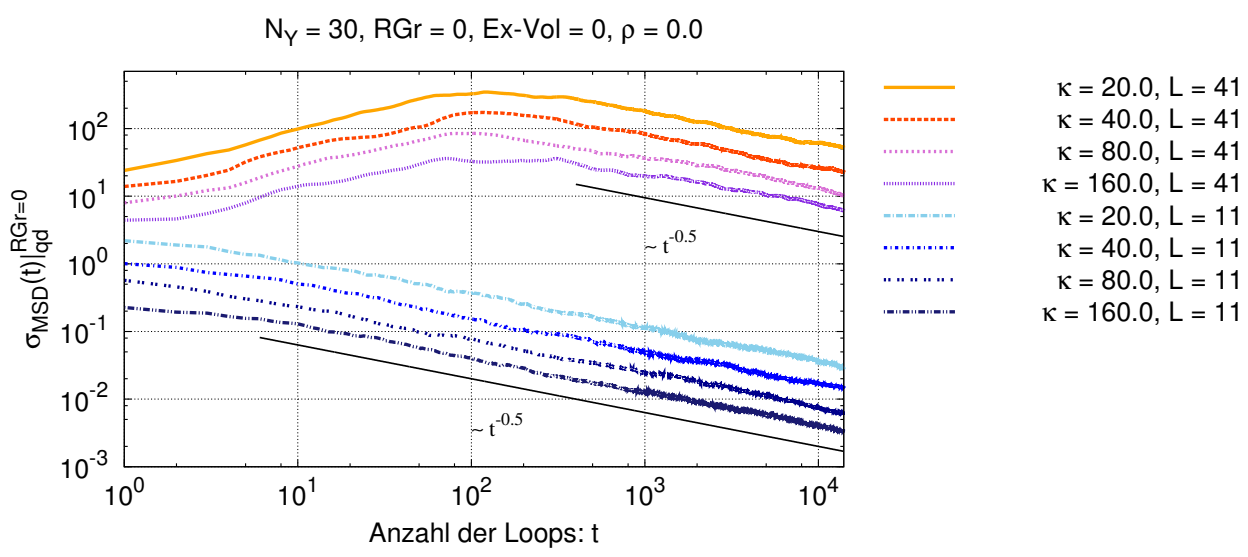

Abbildung 2.12.: MSD-Schwankung $\left.\sigma_{M S D}(t)\right|_{\mathrm{qd}} ^{\mathcal{R} G r=0}$ für verschiedene Werte von $\kappa$ und $L$ als Funktion von $t$

Obige Resultate legen nahe, die Simulationen auf relative kleine Systeme zu beschränken, besonders auf hinreichend niedrige Kettenlängen $L$, um im Rahmen geeigneter Simulationszeiten numerische Berechnungen durchführen zu können. Dadurch sind zwar deutliche Beschränkungen in der Dimensionierung des Systems in Kauf zu nehmen, jedoch werden die relevanten physikalischen Größen messbar und die wesentlichen Phänomene an solch relativ kleinen Systemen ersichtlich sein.

\subsection{Der statische Strukturfaktor $S(s, q)$}

In diesem Kapitel kommen wir erneut auf den bereits im vorangegangenen Kapitel 2.9 eingeführten statischen Strukturfaktor zurück. Wir sind an der strukturellen Information der Ketten interessiert, die sich über die Konformation der Kettensegmente bzw. über die räumliche Anordnung der Ketten ableiten lässt. Unser Hauptaugenmerk richtet sich dabei auf die Korrelation der Segmente bei festem Segmentindex $s$ und messen daher die zeitabhängigen Segmentpositionen $y_{s, i}\left(t^{\prime}\right)$.

Wie Momentaufnahmen (die auch als Schnappschüsse bezeichnet werden) der Polymerkonformationen aller $N_{Y}$ Ketten zu einem festen, aber beliebigen Zeitpunkt $t^{\prime}$ im thermischen Gleichgewicht offenlegen, gibt es in Abhängigkeit der Crosslinkdichte $\rho$ strukturelle Unterschiede, die auch leicht im Ortsraum erkennbar sind (siehe Abbildungen 2.20 und 2.21) in Kapitel 2.16).

Mit einem geringen Crosslink-Anteil erreicht man lediglich eine kleine Kopplung und damit nur eine geringe Wechselwirkung zwischen den Ketten. Aus diesem Grund lässt sich nur eine schwache Korrelation nachweisen, da die Ketten noch weitestgehend unabhängig voneinander Fluktuationsbewegungen ausführen. Steigert man den CrosslinkAnteil jedoch, so wird die Wechselwirkung zwischen den Ketten wachsen und es bilden sich zunehmend Polymerbündel-Strukturen heraus.

Dieser Effekt der Bündelbildung ist visuell leicht im Ortsraum erkennbar, kann je- 


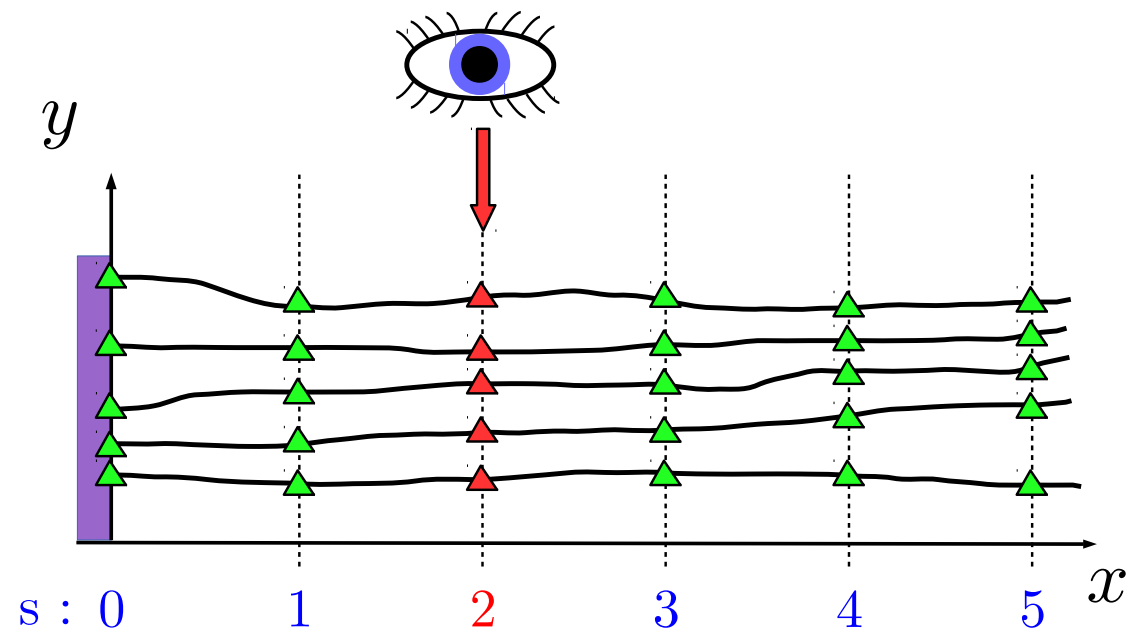

Abbildung 2.13.: Skizzierte Darstellung des segmentweisen Scan-Prozesses zur Untersuchung des Strukturfaktors, hier für $S(s=2, q)$

doch auch im reziproken Raum mit Hilfe der Fouriertransformierten der ZweipunktKorrelationsfunktion, dem statischen Strukturfaktor $S(s, q)$, charakterisiert werden 2, 48. Dieser eignet sich besonders zur Charakterisierung des Systems auf mittleren und größeren Längenskalen.

Im Fokus unseres Interesses liegt die segmentweise strukturelle Information, das heißt bei der Untersuchung von $S(s, q)$ wird der Segmentindex $s$ festgehalten und es werden alle $N_{Y}$ Segmentpositionen $y_{s, i} ; i=1,2, \ldots, N_{Y}$ für die Berechnung herangezogen. Dieses Vorgehen wird für alle $s$ durchgeführt. Die Vorgehensweise ist schematisch in Abbildung (2.13) skizziert. Im dort dargestellten Fall werden die Segmentkoordinaten $y_{2, i}$ zur Ermittlung von $S(s=2, q)$ eingelesen.

Im Impulsraum drücken wir die Teilchendichte in Form der Fouriertransformierten aus 2,48 :

$$
\rho(s, q)=\int n(y(s)) \exp \left(i q y_{s, i}\right) \mathrm{d} y=\sum_{i=1}^{N_{Y}} \exp \left(i q y_{s, i}\right),
$$

mit der Teilchendichte im Ortsraum $n(y(s))=\sum_{i=1}^{N_{Y}} \delta\left(y(s)-y_{s, i}\right)$. Aufgrund der periodischen Randbedingungen in $y$-Richtung sind die Werte des Wellenvektors $q$ quantisiert und hängen von der Länge der Substratoberfläche ab. Daher gilt

$$
q=\frac{2 \pi}{N_{Y}} \cdot n ; n=1,2, \ldots, n_{\max }
$$

mit der beliebig festgelegten maximalen Quantenzahl $n_{\max }=2 \cdot N_{Y}$. Der statische Strukturfaktor berechnet sich unter Berücksichtigung der thermischen Mittelung der reziproken Dichte 2]:

$$
S(s, q)=\frac{1}{N_{Y}}[\langle\rho(s, q) \rho(s,-q)\rangle-\langle\rho(s, q)\rangle\langle\rho(s,-q)\rangle] .
$$


Die schließlich aufzutragende Größe bezeichnen wir mit $S_{\mathrm{AVG}}(q)$ und ist eine Mittelung sowohl über zufällig ermittelte Grafting-Positionen (Quenched Disorder-Mittelwert) als auch eine Mittelung über die letzten $\left(L-s_{c}\right)$ Segmente am freien Kettenende, mit einem Grenz-Index $s_{c},\left(0<s_{c}<L\right)$. Die letztere Mittelung wurde vorgenommen, um eine bessere Statistik anhand der Information von mehreren Strukturfaktoren am freien Ende einfließen zu lassen. So erhalten wir mit $s_{c}=7$ :

$$
S_{\mathrm{AVG}}(q):=\left.\frac{1}{\left(L-s_{c}\right)} \cdot \sum_{s=s_{c}+1}^{L} S(s, q)\right|_{\mathrm{qd}}, \text { mit }\left.S(s, q)\right|_{\mathrm{qd}}:=\frac{1}{r_{\max }} \cdot \sum_{r=1}^{r_{\max }} S_{r}(s, q) .
$$

In den Abbildungen (2.14) und 2.15) sind die durch die oben genannten Mittelungen

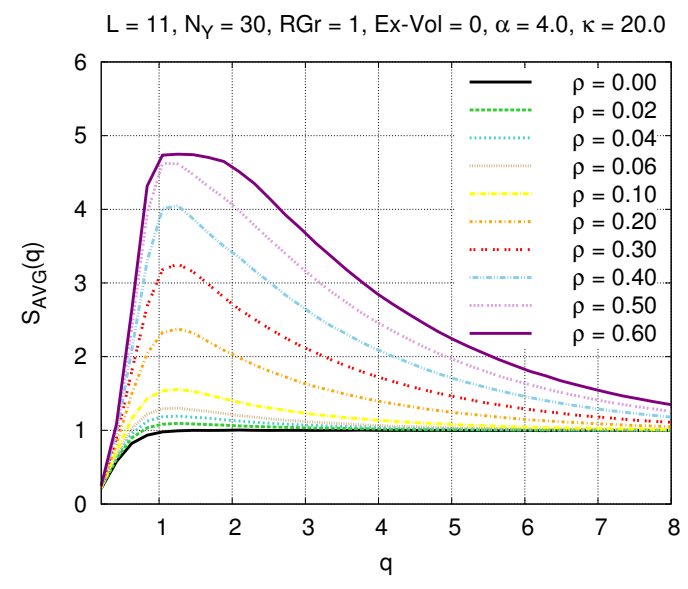

Abbildung 2.14.: $S_{\mathrm{AVG}}(q)$ für verschiedene Crosslink-Dichten $\rho, \alpha=$ konst.

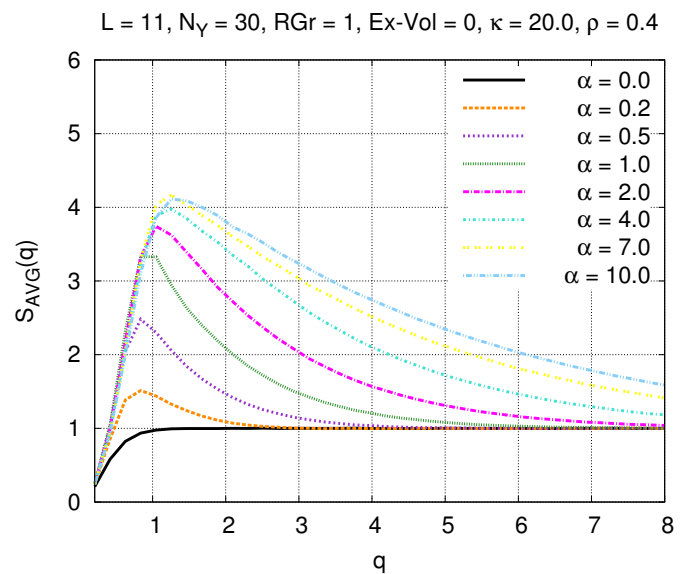

Abbildung 2.15.: $S_{\mathrm{AVG}}(q)$ für verschiedene Crosslink-Stärken $\alpha, \rho=$ konst.

erzeugten Strukturfaktoren $S_{\mathrm{AVG}}(q)$ als Funktion von $q$ aufgetragen. Ohne die Beteiligung von Crosslinks und damit ohne die Wechselwirkung der Ketten untereinander können sich die thermisch fluktuierenden Ketten frei und unabhängig voneinander bewegen. Das bedeutet, dass die Positionen der Segmente nicht miteinander korreliert sind und deshalb die Anordnung der Ketten zu einer vollkommen unregelmäßigen Struktur führt. Folglich ist im Strukturfakor für die beiden Spezialfälle $\rho=0$ oder $\alpha=0$ kein Peak zu beobachten, sondern lediglich ein Untergrundwert von $\left.S_{\mathrm{AVG}}(q>1)\right|_{\rho=0} \approx 1$.

Der statische Strukturfakor ändert sich allerdings grundlegend, wenn Crosslinks in das System eingebracht werden. Die Verknüpfung und die damit verbundene Wechselwirkung der Ketten untereinander führt zu korrelierten und voneinander abhängigen Fluktuationsbewegungen. Je höher der Crosslink-Anteil ist, umso stärker sind die Ketten miteinander gekoppelt, so dass die Korrelation zwischen den Ketten ansteigt. Damit verbunden ist unmittelbar die Entstehung von Polymerbündeln, die mit zunehmendem $\rho$ umso deutlicher in Erscheinung treten.

Selbst sehr geringe Crosslinkzahlen führen bereits zu einer Peak-Bildung, offenbar existiert kein kritischer $\rho$-Wert, um überhaupt eine Bündelbildung zu ermöglichen, sondern es zeigt sich ein kontinuierlicher Phasenübergang zwischen der ungeordneten Phase und der Bündel-Phase. 
Auch der Anstieg der Crosslink-Stärke $\alpha$ führt bei gleichbleibender Crosslink-Dichte zu einem ähnlichen Phänomen. Denn mit einer ansteigenden Federstärke steigt ebenfalls die Kopplungsstärke und damit auch die Korrelation zwischen den Ketten, während eine Abnahme von $\alpha$ mit einer ansteigenden mittleren Crosslink-Länge verbunden ist (siehe Abbildung (2.52) in Kap. 2.23), die im Limes $\alpha \rightarrow 0$ dazu führt, dass die Ketten wieder frei beweglich sind und die Kopplung verschwindet.

$\mathrm{Zu}$ guter Letzt kann man schlussfolgern, dass sowohl mit steigendem Crosslink-Anteil im System als auch mit einer steigenden Crosslink-Stärke eine verstärkte Korrelation der Polymerketten verbunden ist, die zu einer besseren strukturellen Anordnung der Ketten in Form von Bündeln führt, was sich in der Höhe und der Form der Peaks äußert. Je höher die Crosslink-Stärke, umso stärker spalten sich die Bündel in mehrere, kleinere Bündel mit einer deutlicher messbaren, schärferen Struktur auf, im Einklang mit der Verschiebung des Peak-Maximums zu höheren Wellenzahlen $q$.

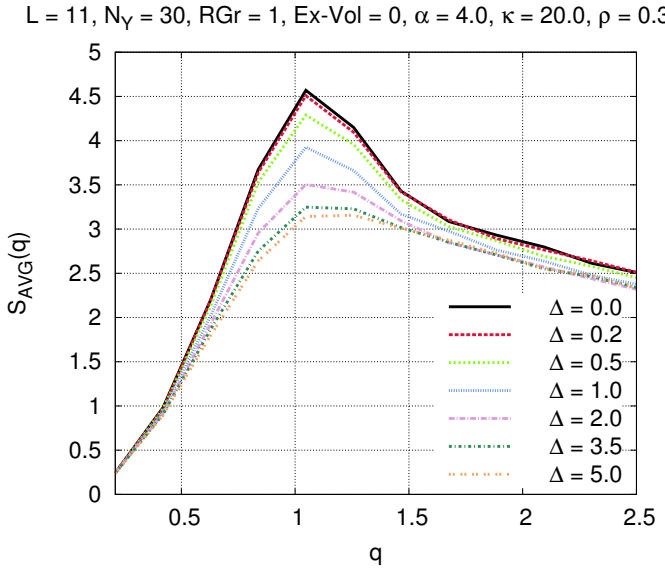

Abbildung 2.16.: $S_{\mathrm{AVG}}(q)$ für verschiedene Intervallbreiten $\Delta$

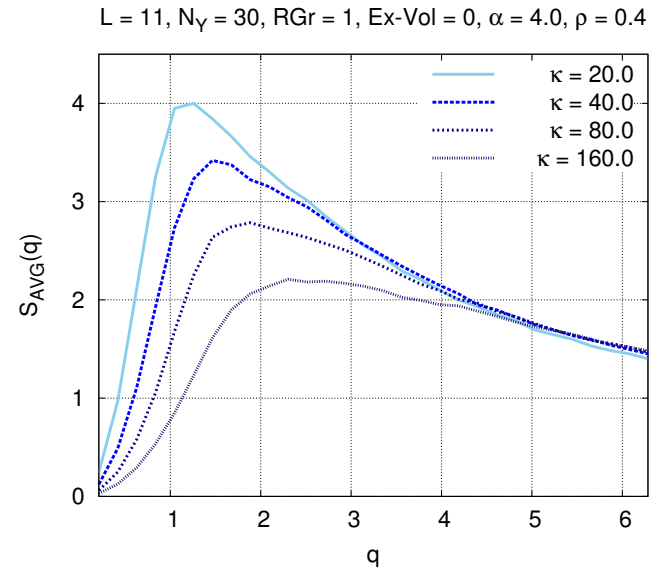

Abbildung 2.17.: $S_{\mathrm{AVG}}(q)$ für verschiedene Biegesteifigkeiten $\kappa$

Zur Ergänzung werden in den Abbildungen (2.16) und (2.17) die Auswirkungen der Variation der Intervallbreite $\Delta$ bzw. der Biegesteifigkeit $\kappa$ auf den Strukturfakor gezeigt. So bildet sich mit steigender Grafting-Ordnung und der damit verbundenen ansteigenden Regelmäßigkeit der Bündelstruktur das Maximum des Strukturfaktors immer deutlicher heraus. Des Weiteren bewirkt die Steigerung der Biegesteifigkeit $\kappa$ die Formation einer höheren Anzahl von Bündeln auf der Substratoberfläche $\mathcal{G}$. Folglich sind die Bündel steifer Ketten kleiner, das heißt sie bestehen im Mittel aus weniger Polymerketten und die Distanz zwischen zwei Bündeln nimmt mit steigendem $\kappa$ ab und das Peak-Maximum wird zu höheren $q$-Werten verschoben (siehe auch Kapitel 2.15).

\subsection{Domänengröße $l^{*}$}

Die Bündelstrukturen lassen sich mit Hilfe der Zweipunkt-Korrelationsfunktion über deren via Fouriertransformation gewonnenen Strukturfakor $S(q)$ charakterisierten. Darüber hinaus kann in diesem Zusammenhang aus dem Strukturfaktor eine Domänengrö- 
ße $l^{*}$ abgeleitet werden [91], die Aufschluss über die mittlere Größe der Bündel gibt.

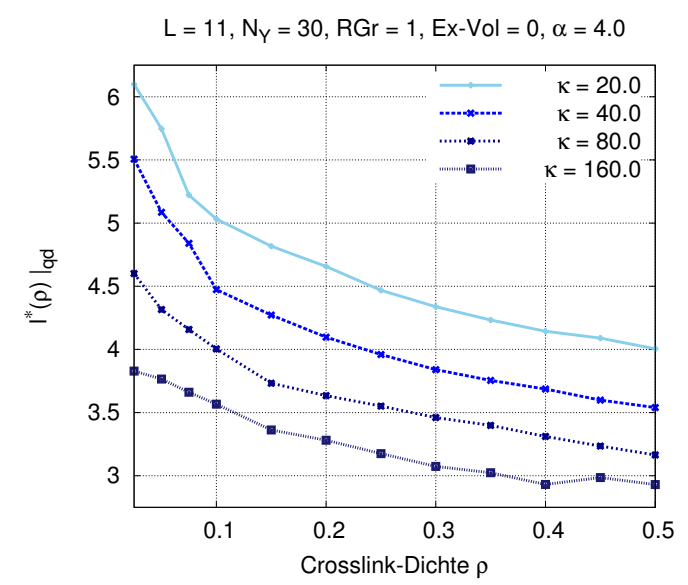

Abbildung 2.18.: $\left.l^{*}(\rho)\right|_{\text {qd }}$ als Funktion von $\rho$ für verschiedene $\kappa$-Werte

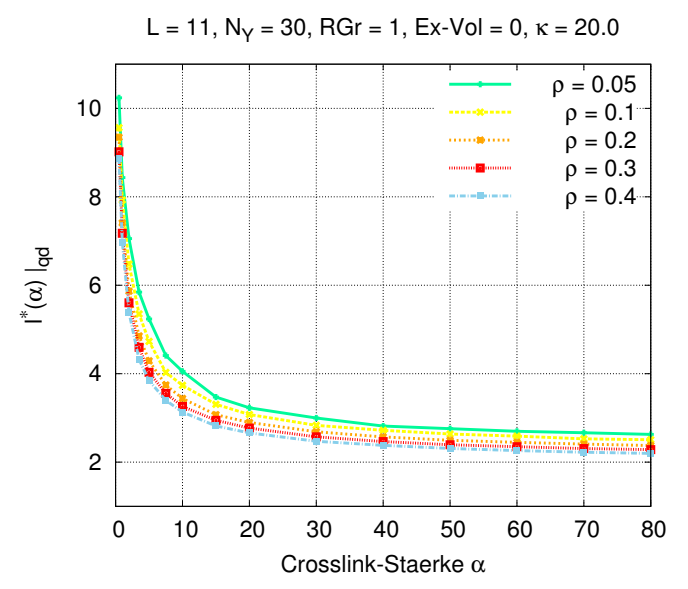

Abbildung 2.19.: $\left.l^{*}(\alpha)\right|_{\text {qd }}$ als Funktion von $\alpha$ für verschiedene $\rho$-Werte

In unserem Fall liegen nach 2.56 diskrete Wellenzahlen vor, das heißt anstelle einer Integration verwenden wir eine Summation über diskrete Wellenzahlen $q$. Zudem können wir lediglich eine endliche Anzahl an Summanden berücksichtigen und legen aus diesem Grund eine Abschneidefrequenz bei $q_{\max }=4 \pi$ fest. Um die Vorgabe zu erfüllen, dass $S_{\mathrm{AVG}}(q) \underset{q \rightarrow \infty}{\longrightarrow} 0$ gilt, wird vom Strukturfaktor der Untergrund wechselwirkungsfreier Ketten $\left(\left.S_{\mathrm{AVG}}(q)\right|_{\rho=0}\right)$ abgezogen, so dass in die Berechnung der Domänengröße ein modifizierter Strukturfakor $\tilde{S}_{\mathrm{AVG}}(q)$ eingeht. Wir berechnen unter Berücksichtigung der obigen Aspekte das erste Moment $q^{*}$ der Funktion $\tilde{S}_{\mathrm{AVG}}(q)$

$$
q^{*}:=\frac{\int_{0}^{\infty} q S(q)_{\mathrm{AVG}} \mathrm{d} q}{\int_{0}^{\infty} S(q)_{\mathrm{AVG}} \mathrm{d} q} \underset{\text { Diskretisierung }}{\longrightarrow}\left(\frac{2 \pi}{N_{Y}}\right) \cdot \frac{\sum_{n=1}^{n_{\max }} n \cdot \tilde{S}_{\mathrm{AVG}}(n)}{\sum_{n=1}^{n_{\max }} \tilde{S}_{\mathrm{AVG}}(n)},
$$

und daraus den Domänenradius bzw. die Domänengröße

$$
l^{*}:=\frac{2 \pi}{q^{*}} .
$$

Die daraus resultierenden Ergebnisse sind in den Abbildungen (2.18) und (2.19) in Abhängigkeit von $\rho$ bzw. von $\alpha$ gegeben. Offensichtlich führt eine Erhöhung sowohl der Crosslink-Stärke als auch der Biegesteifigkeit und der Crosslink-Dichte zu einer Verkleinerung der Domänengröße (vergleiche dazu Kapitel 2.14). Das bedeutet, die Erhöhung dieser drei Parameter begünstigt die Bildung kleinerer Domänen bzw. Bündel.

\subsection{Allgemeine Bemerkungen zur Bündelstruktur}

Zur besseren Veranschaulichung werden in diesem Kapitel die in den beiden vorhergehenden Kapiteln 2.14 und 2.15 mit Hilfe des Strukturfaktors $S_{\mathrm{AVG}}(q)$ charakterisierten Bündelformationen der Polymerketten für zwei verschiedene Parameterkonfigurationen 
im Ortsraum gezeigt (siehe Abbildungen (2.20) und (2.21)). Hier sind die Polymerkonformationen samt den dazugehörigen Crosslinks (symbolisiert durch rote Punkte) in einer Momentaufnahme präsentiert, das heißt wir sehen die Ketten in einem zufällig gewählten Moment bei thermischem Gleichgewicht während der laufenden Simulation.

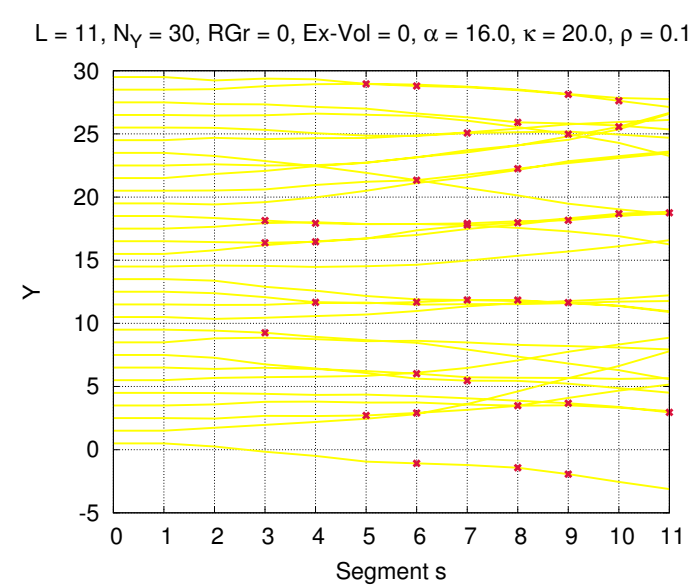

Abbildung 2.20.: Polymerketten mit Crosslinks bei niedriger Crosslink-Dichte $(\rho=0.1)$

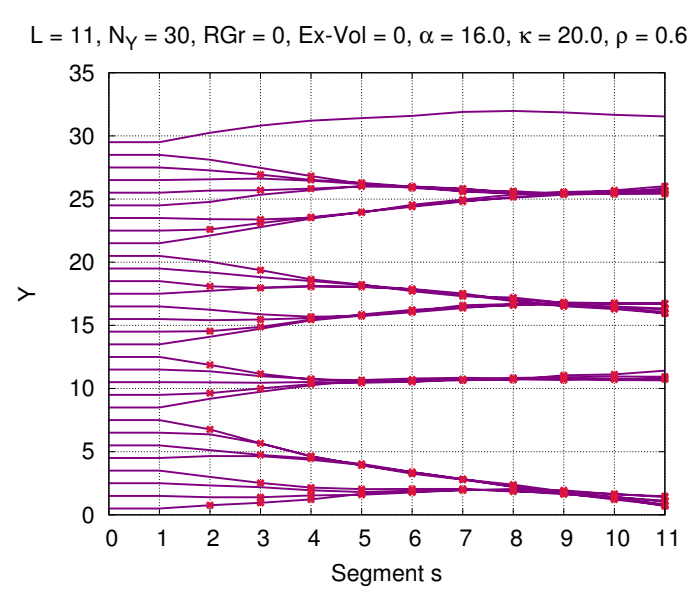

Abbildung 2.21.: Polymerketten mit Crosslinks bei hoher Crosslink-Dichte $(\rho=0.6)$

Bei beiden Schnappschüssen liegen äquidistant gegraftete Ketten vor, der Unterschied zwischen einer niedrigen und einer hohen Crosslink-Dichte wird in der Kettenstruktur deutlich. Während in Abbildung (2.20) aufgrund der nur unzureichend vorhandenen Crosslinks eine relativ ungeordnete Struktur zu erkennen ist, bewirkt eine hohe Zahl an Crosslinks die Entstehung einer deutlich ausgeprägten Bündelstruktur mit sich periodisch wiederholenden Bündelsträngen (Abbildung (2.21)).

Ein interessantes Phänomen mit Hinblick auf die Bündelstruktur wird durch die Veränderung der Grafting-Anordnung der Ketten offenbart. Wie eingangs in Kapitel 2.8 beschrieben, können wir mit Hilfe der Intervallbreite $\Delta$ das Maß der Grafting-Ordnung kontinuierlich variieren. Dabei steht $\Delta=0$ für äquidistant gegraftete Ketten und $\Delta=N_{Y}=30$ für vollkommen zufällig angeordnete Ketten, wobei das Ordnungsmaß aufgrund des mittleren Grafting-Abstandes $\left\langle d_{\mathrm{Gr}}\right\rangle=1$ für $\Delta$-Werte zwischen 0 und 1 relativ schnell abfällt (siehe Kapitel 2.9).

Bei $\mathcal{R} G r=0$ haben wir in $y$-Richtung ein translationsinvariantes System vorliegen, da die Ketten entlang der Grafting-Oberfläche regelmäßig angeordnet sind. Diese Symmetrie der Translationsinvarianz wird aufgehoben, wenn wir mit Hilfe eines von Null verschiedenenen $\Delta$-Wertes unregelmäßige Grafting-Anordnungen erlauben. So stellt sich die Frage, welche Auswirkungen der Wert der Breite $\Delta$ auf das zeitliche Verhalten der Bündel hat.

Wir führen die Größe $a(t)$ ein. Sie gibt die Mittelung des Betrags aller Segmentaus- 
lenkungen $\left\langle u_{\mathrm{Ges}}(t)\right\rangle$, gemittelt über die Simulationszeit $t$ an und ist wie folgt definiert:

$$
a(t):=\frac{1}{t} \int_{0}^{t}\left\langle u_{\mathrm{Ges}}(t)\right\rangle \mathrm{d} t^{\prime} \quad \text { mit } \quad\left\langle u_{\mathrm{Ges}}(t)\right\rangle:=\sum_{i=1}^{N_{Y}} \sum_{s=0}^{L}|\underbrace{\frac{1}{t} \int_{0}^{t} u_{s, i}\left(t^{\prime}\right) \mathrm{d} t^{\prime}}_{\left\langle u_{s, i}(t)\right\rangle}| .
$$

Mit Hilfe der Größe $a(t)$ kann festgestellt werden, ob die Polymerketten im zeitlichen Mittel

- dauerhaft um ihre jeweilige Ruhelage fluktuieren (das heißt, für die beweglichen Segmente gilt dann $\left\langle u_{s, i}(t)\right\rangle \underset{t \rightarrow \infty}{\longrightarrow} 0$ )

oder ob die Ketten im zeitlichen Mittel

- dauerhaft um eine von der jeweiligen Ruhelage abweichenden Lage Fluktuationsbewegungen ausführen (das heißt, für die beweglichen Segmente gilt dann $\left\langle u_{s, i}(t)\right\rangle \underset{t \rightarrow \infty}{\longrightarrow} \tilde{k}_{1}(s, i)$, mit einer von Null verschiedenen, von den Segmentkoordinaten $(s, i)$ abhängigen Konstanten $\left.\tilde{k}_{1}(s, i)\right)$.

Im ersten Fall erwartet man nach dem zentralen Grenzwertsatz eine Proportionalität $a(t) \propto \frac{1}{\sqrt{t}}$. Im zweiten Fall dagegen wird $a(t)$ nicht kontinuierlich mit der Zeit abfallen, sondern für hinreichend große Simulationszeiten einen konstanten, von Null verschiedenen Wert annehmen: $a(t) \underset{t \rightarrow \infty}{\longrightarrow} \tilde{k}_{2}(\alpha, \Delta, \rho) \neq 0$. Diese Größe ist also ein Maß zur Messung einer „eingeforenen Abweichung“ der Kettenkonformationen von der Ruhelage bzw. von der parallelen Ausrichtung bezüglich der $x$-Achse.

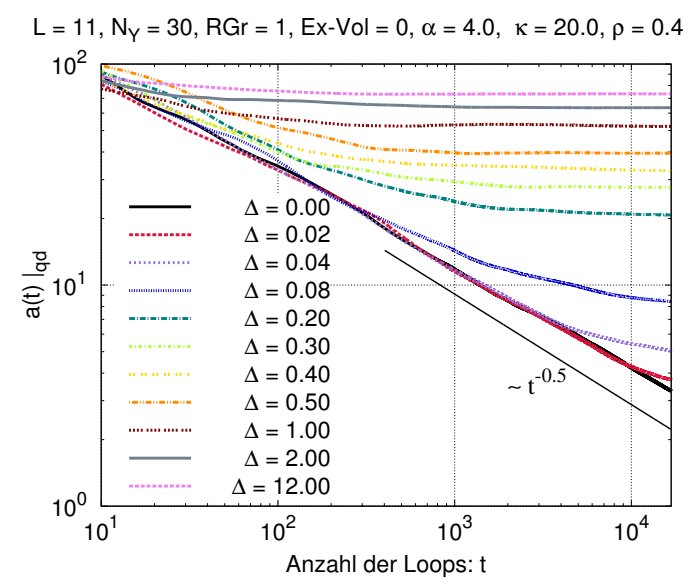

Abbildung 2.22.: $\left.a(t)\right|_{\text {qd }}$ als Funktion der Zeit $t$ für verschiedene $\Delta$-Werte

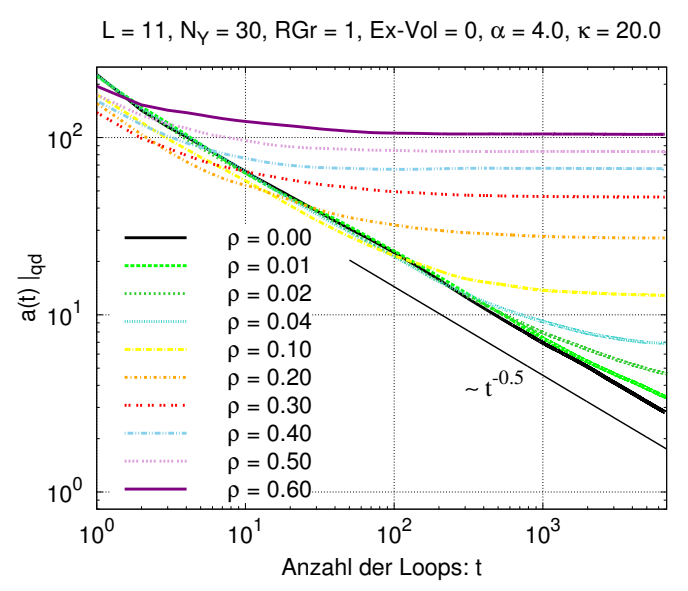

Abbildung 2.23.: $\left.a(t)\right|_{\text {qd }}$ als Funktion der Zeit $t$ für verschiedene $\rho$-Werte

Man kann die Segmentauslenkungen $u_{s, i}(t)$ im Rahmen einer statistischen Beschreibung als Zufallsvariable betrachten. In Kapitel 2.21 wird gezeigt, dass die einzelnen Segmentauslenkungen unabhängiger Ketten in sehr guter Näherung durch eine Gaußsche Wahrscheinlichkeitsverteilung mit Erwartungswert Null beschrieben werden können, das heißt, wir erwarten zumindest bei crosslinkfreien Ketten den ersten Fall mit $a(t) \propto \frac{1}{\sqrt{t}}$. 
Die Abbildungen (2.23) und (2.22) zeigen den Quenched Disorder-Mittelwert von $a(t)$ als Funktion der Simulationszeit $t$ bzw. der Anzahl an Schleifendurchgängen. Wir wissen zum Beispiel durch die Kenntnis des Strukturfaktors $S_{\mathrm{AVG}}(q)$ in Kapitel 2.14 . dass unabhängig von der Grafting-Anordnung mit der Existenz von Crosslinks stets Bündelstrukturen verbunden sind. Damit wird deutlich, dass die Intervallbreite $\Delta$ einen fundamentalen Einfluss auf die Persistenz dieser Bündelstrukturen hat.

Wie in Abbildung (2.22) zu erkennen ist, führen lediglich äquidistant gegraftete Ketten mit $\Delta=0$ dazu, dass die Bündel entlang der $y$-Achse wandern können. Somit ist in diesem Falle der Erwartungswert für eine Segmentauslenkung gleich Null und es gibt keinen bevorzugten Ort zur Bündelbildung. Zwar fluktuieren die Ketten eines Bündels innerhalb eines kleinen Zeitintervalls um eine von der Ruhelage abweichenden Position. Doch wandern diese Bündel, auf einer größeren Zeitskala betrachtet, entlang der $y$ Achse und die Auslenkungen aller Segmente weisen dann keine Vorzugsrichtung mehr auf. Es gilt $\left\langle u_{s, i}\right\rangle=0$ und schließlich $\left.a(t)\right|_{\text {qd }} \propto 1 / \sqrt{t}$ entweder für $\rho=0$ oder $\Delta=0$.

Im Gegensatz dazu haben schon geringfügige Unregelmäßigkeiten in den Kettenabständen mit $\Delta>0$ zur Folge, dass Ketten eine Anordnung jenseits der Ruhelage dauerhaft einnehmen, das heißt, bei nicht exakt äquidistant gegrafteten Ketten können Bündelstrukturen bezüglich der $y$-Achse ortsfest werden. Es gibt bedingt durch variierende Kettenabstände entlang der $y$-Koordinate Orte im System, in denen Bündelbildung begünstigt wird und gebündelte Ketten als Zustand eingefroren werden. Diesen Effekt bezeichnen wir als den Quenched Disorder-Effekt. Dieser wird mit abnehmender Grafting-Ordnung bzw. mit steigendem $\Delta$ verstärkt, das heißt die betragsmäßige Abweichung aller Segmente von der Ruhelage nimmt zu und $a(t)$ nimmt höhere Plateauwerte ein (siehe Abb. (2.22)).

Im Vergleich dazu betrachten wir den Effekt einer steigenden Crosslink-Dichte auf den Quenched Disorder-Mittelwert von $a(t)$ bei stets maximal unregelmäßig gegrafteten Ketten mit $\Delta=30.0$ (siehe Abbildung (2.23)). Es ist festzustellen, dass nur im Fall $\rho=0$ die dann voneinander unabhängigen Ketten alle um ihre Ruhelange fluktuieren, so dass $\left\langle u_{s, i}\right\rangle \rightarrow 0$ erfüllt wird. Werden im Unterschied dazu Ketten mit Crosslinks verknüpft, entstehen ortsfeste Bündel, deren Struktur sich mit wachsendem $\rho$ immer stärker herausbildet, was sowohl im Strukturfaktor als auch in der zunehmenden Abweichung der beweglichen Segmente von der Ruhelage sichtbar wird. Dies geht mit einem wachsenden Plateauwert von $a(t)$ einher.

Eine alternative Methode zur Messung des Quenched Disorder-Effektes wird im Anhang A.7 erläutert.

Zur anschaulichen Darstellung von eingefrorenen, persistenten Kettenstrukturen im Ortsraum eignet sich die Messung der zeitlich gemittelten Segmentpositionen im thermischen Gleichgewicht:

$$
\left\langle\boldsymbol{r}_{s, i}\right\rangle:=\left(b \cdot s, g_{i}+\left\langle u_{s, i}\right\rangle\right) .
$$

Diese zeitlich gemittelten Segmentpositionen zeigen die Abbildungen (2.24) und 2.25) für zwei unterschiedlich stark geordnete Grafting-Konfigurationen. Wie bereits erläutert, führen äquidistant gegraftete Ketten $(\Delta=0)$ aufgrund der Translationsinvarianz in $y$-Richtung dazu, dass der Erwartungswert der Segmentauslenkungen im thermischen Gleichgewicht verschwindet und für die zeitlich gemittelten Segmentpositionen 


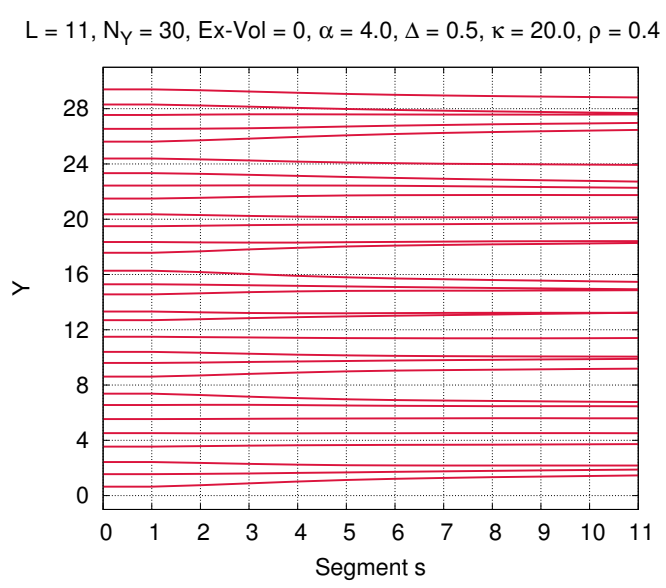

Abbildung 2.24.: mittlere Positionen $\left\langle\boldsymbol{r}_{s, i}\right\rangle$ für $\Delta=0.5$ (hohe Grafting-Ordnung)

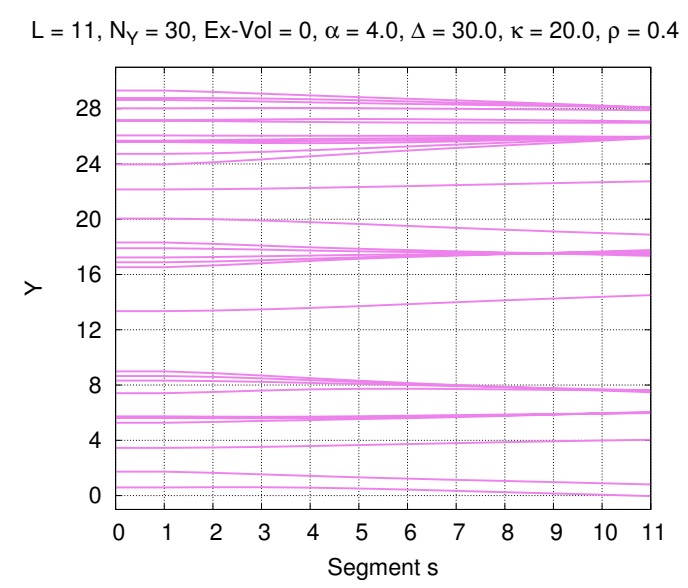

Abbildung 2.25.: mittlere Positionen $\left\langle\boldsymbol{r}_{s, i}\right\rangle$ für $\Delta=30.0$ (niedrige Grafting-Ordnung)

deshalb $\left.\left\langle\boldsymbol{r}_{s, i}\right\rangle\right|_{\Delta=0} \underset{t \rightarrow \infty}{\longrightarrow}\left(b \cdot s, g_{i}\right)$ gilt. Wenden wir uns der Grafting-Konfiguration mit der hohen Grafting-Ordnung zu ( $\Delta=0.5$, Abb. (2.24)), so fällt auf, dass sich persistente Bündelstrukturen nur in geringem Maße herausgebildet haben. Entlang der Grafting-Oberfläche besteht eine nur geringe Heterogenität bezüglich der Präferenz von Orten zur Bündelbildung, so dass Bündel im zeitlichen Mittel fast überall gleichmäßig oft erscheinen können.

Im Gegensatz dazu führt eine Grafting-Konfiguration mit einer niedrigen Grafting-Ordnung $(\Delta=30.0)$ zu einer starken Heterogenität entlang der $y$-Richtung bzw. zu einer großen Schwankung in den Abständen benachbarter Ketten. Damit gibt es auf der Grafting-Oberfläche Stellen, die ausgeprägte und persistente Bündelstrukturen ermöglichen und aufweisen. Die eingefrorenen Bündelstrukturen sind durch eine deutliche Abweichung der zeitlich gemittelten Kettenkonformationen von der jeweiligen Ruhelage der Polymerketten erkennbar.

Ein Maß zur Quantifizierung der persistenten Abweichung der Kettenenden $(s=L)$ aus der Ruhelage lässt sich mit Hilfe von

$$
Q_{D}:=\frac{1}{N_{Y}} \sum_{i=1}^{N_{Y}}\left(\left\langle u_{L, i}\right\rangle\right)^{2} \quad \text { mit } \quad\left\langle u_{L, i}\right\rangle:=\lim _{t \rightarrow \infty} \int_{0}^{t} u_{L, i}\left(t^{\prime}\right) \mathrm{d} t^{\prime}
$$

angeben. Sind die Bündel nicht persistent, das heißt, können die Bündelstrukturen entlang der $y$-Achse wandern, so erwartet man in diesem Falle einen verschwindenen $Q_{D}$-Wert. Andernfalls, bei einer eingefrorenen Bündelstruktur, gibt es Ketten, die dauerhaft eine Position fern der Ruhelage mit $\left\langle u_{s, i}\right\rangle \neq 0$ einnehmen. Wir erwarten einen umso höheren numerischen Wert von $Q_{D}$, je stärker der Quenched DisorderEffekt ausgeprägt ist.

Die Abhängigkeit der Größe $Q_{D}$ von der Crosslink-Stärke $\alpha$ bzw. von der Intervallbreite $\Delta$ ist in den Abbildungen (2.26) und (2.27) aufgetragen. Hier ist deutlich sichtbar, welchen Einfluss die Zunahme der Grafting-Unordnung auf die Größe der dauerhaften 


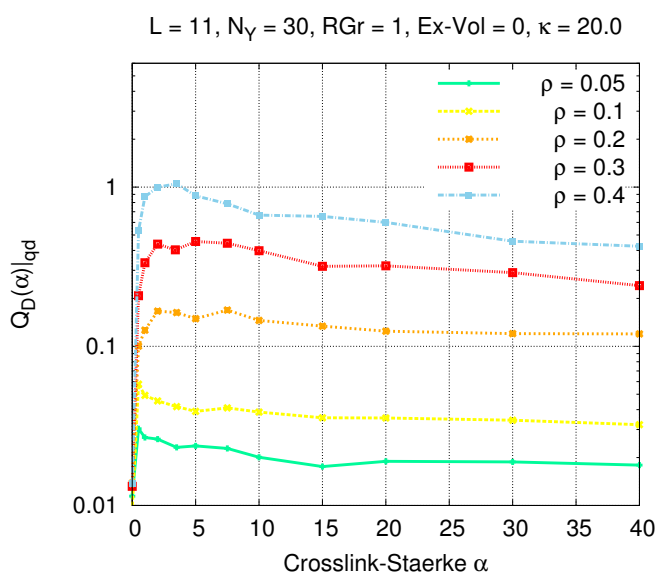

Abbildung 2.26.: $\left.Q_{D}(\alpha)\right|_{\text {qd }}$ als Funktion von $\alpha$ für verschiedene $\rho$-Werte

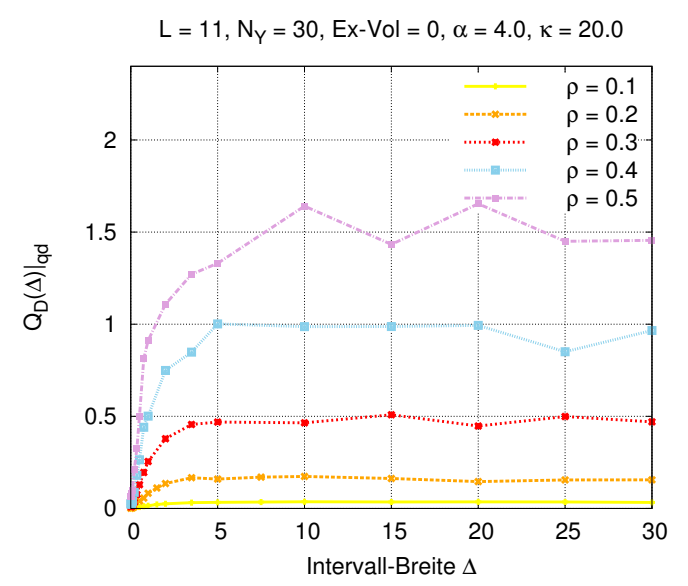

Abbildung 2.27.: $\left.Q_{D}(\Delta)\right|_{\text {qd }}$ als Funktion von $\Delta$ für verschiedene $\rho$-Werte

Abweichungen der Kettenpositionen aus ihrer Ruhelage hat. Dieser Quenched Disorder-Effekt wird erwartungsgemäß mit zunehmender Crosslinkzahl bzw. zunehmender Kopplung zwischen den Ketten verstärkt. Im Falle zufällig verteilter Grafting-Positionen $(\Delta=30.0)$ bewirkt erst eine endliche, positive Crosslink-Stärke $\alpha$ die Einstellung einer Quenched Disorder-Struktur. Zu erkennen ist außerdem, dass $\left.Q_{D}(\alpha)\right|_{\mathrm{qd}}$ ab einer gewissen Crosslink-Stärke nur noch geringfügig von $\alpha$ abhängt.

\subsection{Memory-Effekt}

In diesem Kapitel gehen wir kurz auf ein weiteres interessantes Phänomen ein, das das System im thermischen Gleichgewicht charakterisiert. Es zeigt sich, dass Ketten mit einer festen, zufällig gewählten Grafting-Anordnung (das heißt der Grafting-Seed ist konstant) unabhängig von der Startkonfiguration bzw. der Anfangsauslenkung der Segmente und unabhängig von der Zufallsverteilung der Crosslinks im Grenzfall $t \rightarrow \infty$ stets die identische Kettenkonformation einnehmen, um welche die Segmente thermische Fluktuationen ausführen. Dies ist ein Hinweis auf die Existenz eines sogenannten Memory-Effektes [116]. Mit einer fest vorgegebenen Menge an Grafting-Points $\left\{g_{0}, \ldots, g_{N_{Y}}\right\}$ ist stets eine eindeutige Struktur verbunden, zu der die Ketten im thermischen Mittel tendieren. Beim Speziallfall äquidistant gegrafteter Ketten mit $\Delta=0$ gibt es, wie bereits erwähnt, keine eingefrorene Bündelstruktur und die Ketten streben in der Langzeitmittelung eine Ausrichtung senkrecht zur Substratoberfläche an.

Zur Verifizierung des Memory-Effektes lassen wir in $r_{\max }=10$ unabhängigen Runs ein System mit einer festen, zufällig gewählten Grafting-Konfiguration MC-Schritte ausführen, das heißt, die Runs unterscheiden sich nur in der Vorgabe der Anfangskonfiguration der Ketten und den Anfangspositionen der Crosslinks. Während der Simulation werden die über die Zeit gemittelten $r_{\max }$ Positionswerte $\left\langle y_{s, i}^{r}(t)\right\rangle=g_{i}+\left\langle u_{s, i}^{r}(t)\right\rangle$; $r=1,2, \ldots, r_{\max }$ in äquidistanten Zeitabständen gespeichert. Für jedes einzelne Segment kann damit die Schwankung der $\left\langle y_{s, i}^{r}(t)\right\rangle$-Werte als Funktion der Zeit ermittelt 


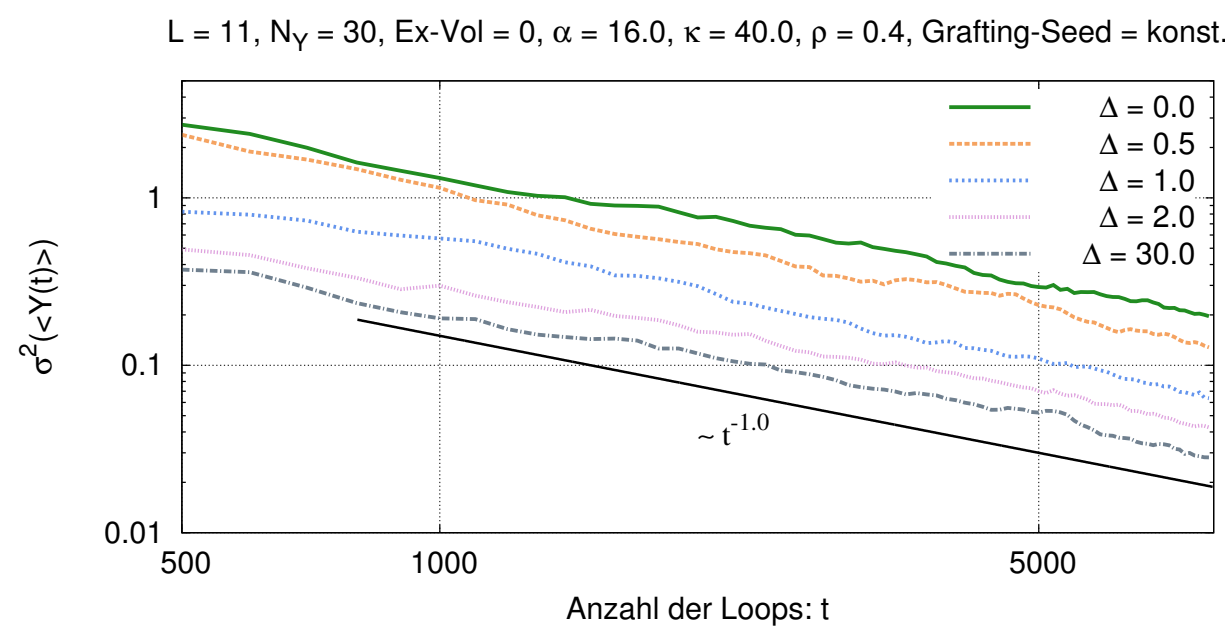

Abbildung 2.28.: Varianz $\sigma^{2}(\langle Y(t)\rangle)$ als Funktion von $t$ für verschiedene Breiten $\Delta$

werden. Daraus kann schließlich die Summe der Varianzen aller zeitlich gemittelten Positionswerte aus den $r_{\max }$ Simulationsläufen als Funktion der Zeit $t$ abgeleitet werden:

$$
\sigma^{2}(\langle Y(t)\rangle):=\sum_{i=1}^{N_{Y}} \sum_{s=0}^{L} \sigma^{2}\left(\left\langle y_{s, i}(t)\right\rangle\right)=\sum_{i=1}^{N_{Y}} \sum_{s=2}^{L} \sigma^{2}\left(\left\langle y_{s, i}(t)\right\rangle\right),
$$

mit den Varianzen der zeitlich gemittelten Segmentpositionen:

$$
\sigma^{2}\left(\left\langle y_{s, i}(t)\right\rangle\right)=\left(\frac{1}{r_{\max }} \sum_{r=1}^{r_{\max }}\left(\left\langle y_{s, i}^{r}(t)\right\rangle\right)^{2}\right)-\left(\frac{1}{r_{\max }} \sum_{r=1}^{r_{\max }}\left(\left\langle y_{s, i}^{r}(t)\right\rangle\right)\right)^{2} .
$$

Abbildung 2.28 veranschaulicht die Existenz des Memory-Effektes. Wir sehen eine gemäß des zentralen Grenzwertsatzes mit der Simulationszeit $t$ abfallende Varianz $\sigma^{2}(\langle Y(t)\rangle) \propto \tilde{b} / t$, das heißt mit fortschreitender Zeit nähern sich die Werte der gemittelten Segmentpositionen immer weiter an, die Konformationen aller $N_{Y}$ Ketten münden also in dieselbe Quenched Disorder-Struktur. Ein interessanter Aspekt dabei ist, dass der Proportionalitätsfaktor $\tilde{b}$ mit der Intervallbreite $\Delta$ skaliert. Je niedriger die Grafting-Ordnung, desto geringer sind die Schwankungen bei einer fest gewählten Zeit $t$.

Zur besseren visuellen Veranschaulichung des Memory-Effektes sind in Abbildung (2.29) die gemittelten Segmentpositionen eines bestimmten Systemausschnitts gezeigt. Anhand von vier beliebig gewählten Zeitpunkten kann deren Entwicklung für vier Runs nachvollzogen werden. Man erkennt leicht, dass mit zunehmender Simulationsdauer die Abweichungen zwischen den gemittelten Positionen der verschiedenen Runs immer weiter abnehmen. 

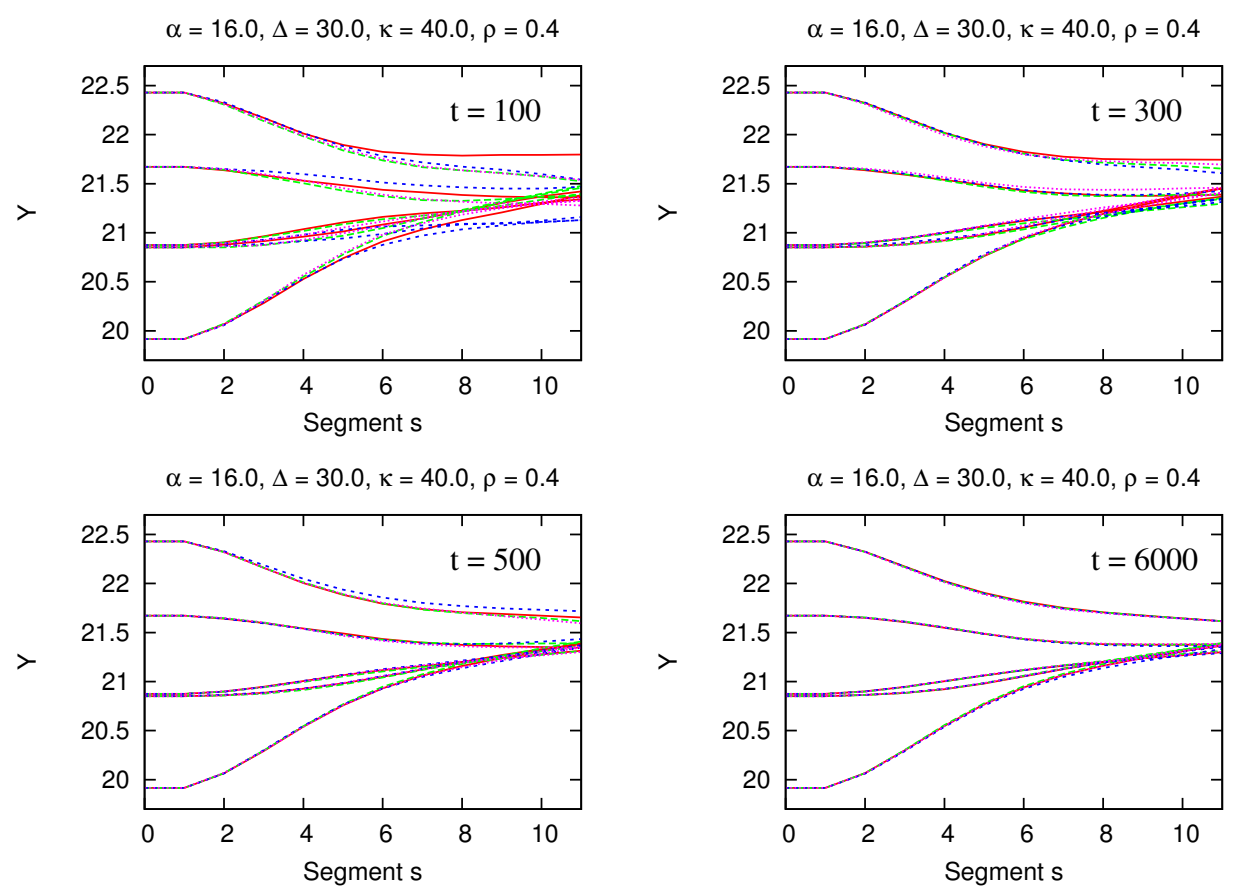

Abbildung 2.29.: $\left\langle\boldsymbol{r}_{s, i}(t)\right\rangle$ für verschiedene Zeiten $t$

\subsection{Mittlere Biege- und Gesamtenergie}

In diesem Kapitel stellen wir den Einfluss der in Kapitel 2.8 eingeführten Parameter $\alpha, \kappa$ und $\rho$ auf verschiedene Energiebeiträge dar. Wie der Hamiltonian 2.48 verdeutlicht, können wir die Gesamtenergie des Systems $E_{\text {Ges }}$ in einen Crosslink-Anteil $E_{\text {xlink }}$ und einen Ketten-Anteil $E_{\kappa}$ aufteilen. Wir geben die Energien im thermischen Equilibrium pro Polymerkette an, das heißt, wir schreiben für alle genannten Energiebeiträge (das Symbol „০" steht wahlweise für "Ges" oder „,")

$$
E_{\circ} \equiv \frac{\left\langle\mathcal{H}_{\circ}\right\rangle}{N_{Y}} \quad \text { mit } \quad\left\langle\mathcal{H}_{\circ}\right\rangle=\lim _{t \rightarrow \infty} \frac{1}{t} \int_{0}^{t} \mathcal{H}_{\circ}\left(t^{\prime}\right) \mathrm{d} t^{\prime},
$$

und dem von den instantanen Kettenkonformationen oder Crosslink-Positionen zum Zeitpunkt $t^{\prime}$ abhängigen Hamiltonian $\mathcal{H}_{\circ}\left(t^{\prime}\right)$. Zuerst betrachten wir die mittlere Biegeenergie pro Kette $E_{\kappa}$ als Funktion der Crosslink-Stärke $\alpha$ bzw. der Biegesteifigkeit $\kappa$ bei einer konstanten Crosslink-Dichte $\rho=0.4$ und verwenden dabei äquidistant gegraftete Polymerketten $(\mathcal{R} G r=0)$ (siehe Gleichung (2.18) sowie Abbildungen (2.30) und (2.31).

Die Daten zeigen, dass eine Erhöhung der Biegesteifigkeit eine Zunahme der Biegeenergie mit sich führt, sofern die Crosslinks eine hinreichend hohe Crosslink-Stärke von $\alpha \approx 1$ aufweisen. Außerdem hat eine Zunahme der Crosslink-Stärke $\alpha$ hat eine Auffächerung der Energiekurven gleicher Biegesteifigkeit zur Konsequenz. Bei zu weichen Crosslinks hingegen ist $E_{\kappa}$ aufgrund des dann nur geringen Crosslink-Einflusses nahezu unabhängig von der Biegesteifigkeit, woraus niedrige Plateauwerte folgen (siehe 


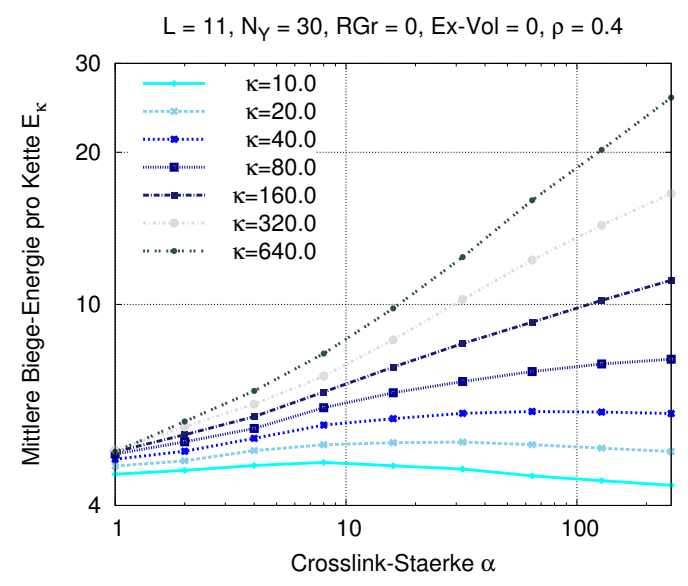

Abbildung 2.30.: Mittlere Biegeenergie pro Kette $E_{\kappa}$ als Funktion von $\alpha$ für verschiedene $\kappa$-Werte

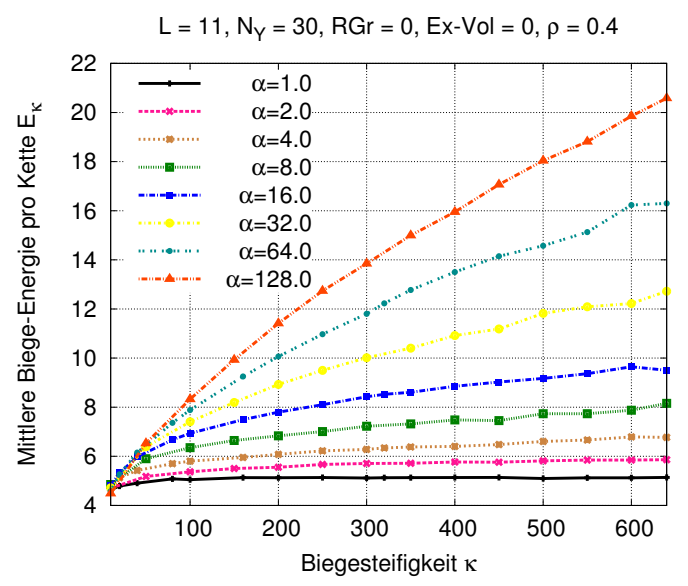

Abbildung 2.31.: Mittlere Biegeenergie pro Kette $E_{\kappa}$ als Funktion von $\kappa$ für verschiedene $\alpha$-Werte

Abb. 2.31). Während ab einem kritischen $\kappa$-Wert die Biegeenergie stets monoton mit $\alpha$ steigt, kann bei zu flexiblen Ketten die Biegeenergie mit wachsendem $\alpha$ sogar wieder abnehmen (siehe Abb. 2.30).

In den Abbildungen 2.32 und 2.33 sind die mittleren Gesamtenergien $E_{\text {Ges }}$ bei fester Crosslink-Stärke $\alpha$ in Abhängigkeit von $\kappa$ bzw. von $\rho$ aufgetragen, auch hier gilt $\mathcal{R} G r=0$. Es lässt sich festhalten, dass sowohl mit der Zunahme der Crosslink-Dichte als auch mit der Zunahme der Biegesteifigkeit eine nichtlineare Erhöhung der Gesamtenergie verbunden ist. Die nichtlineare Erhöhung von $E_{\text {Ges }}$ durch das Hinzufügen von Crosslinks hängt mit der Bildung von Bündeln zusammen. So wird der Zuwachs von $E_{\text {Ges }}$ mit zunehmenden $\kappa$-Werten umso stärker, je mehr Crosslinks sich im System befinden.

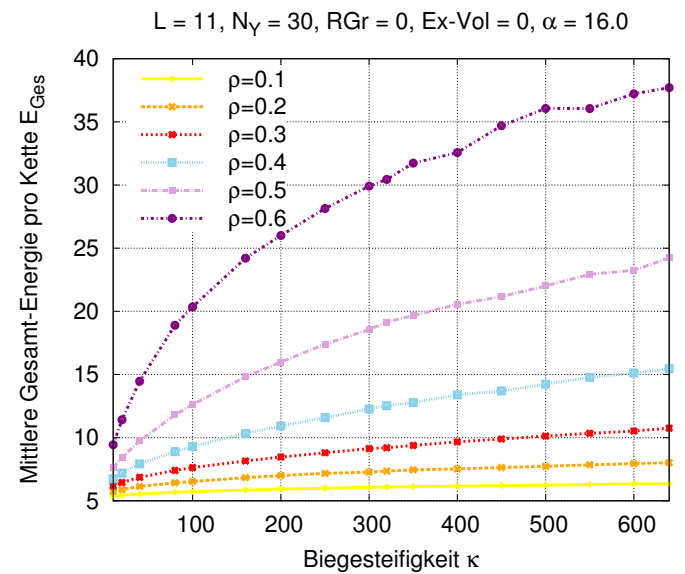

Abbildung 2.32.: Mittlere Gesamtenergie pro Kette $E_{\text {Ges }}$ als Funktion von $\kappa$ für verschiedene $\rho$-Werte

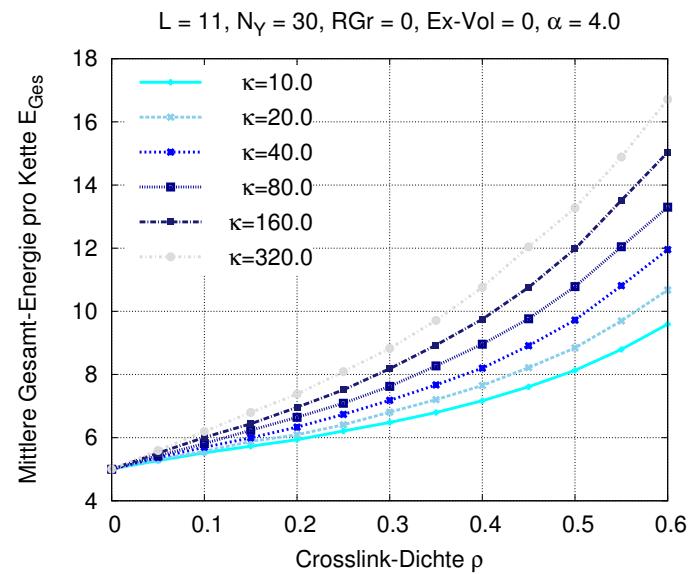

Abbildung 2.33.: Mittlere Gesamtenergie pro Kette $E_{\text {Ges }}$ als Funktion von $\rho$ für verschiedene $\kappa$-Werte 
Bei den obigen Energiebetrachtungen hatten wir stets äquidistant gegraftete Ketten mit $\mathcal{R} G r=0$ vorliegen. Eine sich stellende Frage ist, wie sich die Variation der Intervallbreite $\Delta$ auf die Gesamt- und Biegeenergie auswirkt. Dass auch die GraftingOrdnung einen nicht unerheblichen Einfluss auf die Energien des Systems haben, zeigen die Abbildungen (2.34) und (2.35). Hier sind die über $r_{\max }=20$ gemittelten Quenched Disorder-Mittelwerte der mittleren Gesamt- bzw. Biegeenergien des Systems als Funktion von $\Delta$ für unterschiedliche Crosslink-Dichten $\rho$ angegeben.

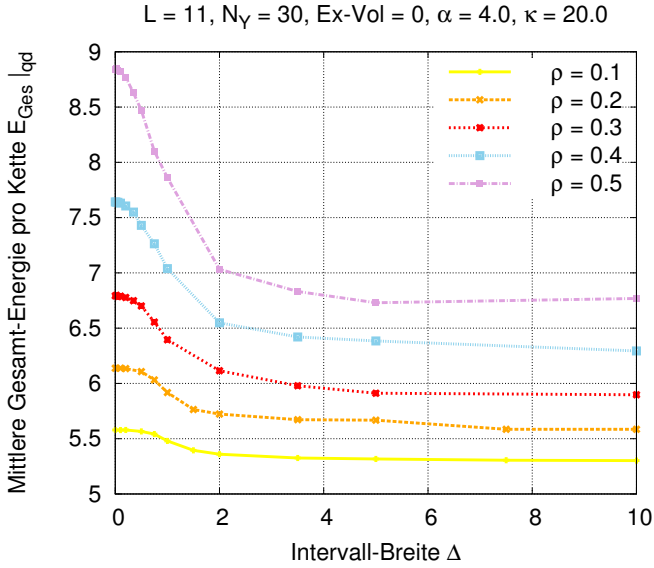

Abbildung 2.34.: Mittlere Gesamtenergie pro Kette $\left.E_{\text {Ges }}\right|_{\text {qd }}$ als Funktion von $\Delta$ für verschiedene $\rho$-Werte

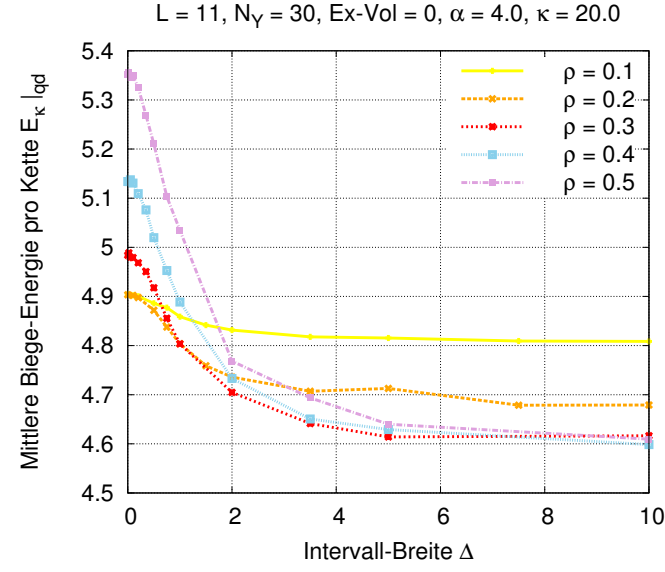

Abbildung 2.35.: Mittlere Biegeenergie pro Kette $\left.E_{\kappa}\right|_{\text {qd }}$ als Funktion von $\Delta$ für verschiedene $\rho$-Werte

Die Diagramme zeigen eine Abnahme sowohl der Biegeenergie $\left.E_{\kappa}\right|_{\text {qd }}$ als auch der Gesamtenergie $\left.E_{\text {Ges }}\right|_{\text {qd }}$ mit steigender Intervallbreite bzw. mit zunehmender GraftingUnordnung. Dabei ist die Energieabnahme der Biege- und der Gesamtenergie mit wachsendem $\Delta$ umso größer, je mehr Crosslinks im System enthalten sind. Dieser Effekt zeigt ebenfalls die mit steigendem $\rho$ wachsende kollektive Wechselwirkung der Ketten, die ohne Excluded Volume-Wechselwirkung keine weitere Interaktionsmöglichkeit besitzen. Im Grenzfall crosslinkfreier Ketten liegen unabhängige Ketten vor. Die Grafting-Anordnung spielt dann keine Rolle mehr und die Energien sind unabhängig von $\Delta$.

Die Zunahme der Grafting-Unordnung ermöglicht eine Anordnung der Crosslinks bevorzugt in der Art, dass im thermischen Gleichgewicht im Mittel weniger Crosslinkund auch weniger Biegeenergie aufgewendet werden muss.

\subsection{Richtungsabhängige Korrelation der Segmente}

Bei semiflexiblen Polymerketten mit einer endlichen Biegesteifigkeit $\kappa$ sind im Unterschied zu einer Freely Jointed Chain [23] die einzelnen Bond-Vektoren $\boldsymbol{t}_{s}$ derselben Kette miteinander korreliert, wobei diese Korrelation mit zunehmender Persistenzlänge $L_{p}$ verstärkt wird (siehe Kapitel 2.4. Wie erläutert wurde, ist in unserem Modell eine Knäuelbildung unabhängig vom Wert der Biegesteifigkeit ausgeschlossen, da die 
Segmente lediglich entlang der $y$-Achse ausgelenkt werden können. Ein Bond-Vektor weist demzufolge immer eine in positive $x$-Richtung zeigende $x$-Komponente auf. Wir erkennen unmittelbar, dass für den Winkel $\theta_{s}$ zwischen der Substratoberfläche und einem Bond-Vektor $\boldsymbol{t}_{s}$ ausschließlich Werte aus dem Intervall $(0, \pi)$ möglich sind. Bei hinreichend steifen Ketten allerdings sind nur kleine Auslenkungen aus der Ruhelage möglich und wir erhalten dabei schmale, gaußförmige Winkelverteilungen um den Mittelwert $\theta=\frac{\pi}{2} \quad$ (siehe Kapitel 2.21).

Aus dem Hamiltonian der Wormlike Chain (2.6) kann das exponentielle Abklingen der Tangens-Korrelationsfunktion für das thermische Gleichgewicht analytisch berechet werden [46, 75] (siehe Gleichung (2.8)). Übertragen wir dies auf den diskreten Fall, verwenden wir anstelle des Tangenten-Einheitsvektors $\hat{\boldsymbol{t}}(s)$ ein diskretes Analogon, nämlich den Einheits-Bond-Vektor $\hat{\boldsymbol{t}}_{s, i}=\frac{\boldsymbol{t}_{s, i}}{\left|\boldsymbol{t}_{s, i}\right|}$. Für eine frei bewegliche Einzelkette ohne Wechselwirkungen mit anderen Ketten führt dies zu 64

$$
\left\langle\hat{\boldsymbol{t}}_{s_{1}, i} \cdot \hat{\boldsymbol{t}}_{s_{2}, i}\right\rangle=\exp \left(-\frac{\left|s_{1}-s_{2}\right| \cdot b}{L_{p}}\right) .
$$

Wir untersuchen die Richtungskorrelation zwischen den beweglichen Bond-Vektoren und dem feststehenden, rechtwinklig zur Grafting-Oberfläche aufgepfropften BondVektor $\boldsymbol{t}_{0, i}$, das heißt, wir sind am mittleren Cosinus des Winkels zwischen dem ersten und dem $(s+1)$-ten Bond-Vektor $\boldsymbol{t}_{s, i}$ interessiert $\left(\left\langle\cos \theta_{s, i}\right\rangle\right)$. Aus geometrischen Überlegungen ergibt sich für eine Kette $i$ zum Zeitpunkt $t^{\prime}$

$$
\left(\hat{\boldsymbol{t}}_{0, i} \cdot \hat{\boldsymbol{t}}_{s, i}\right)\left(s, i, t^{\prime}\right)=\frac{\boldsymbol{t}_{0, i}\left(t^{\prime}\right) \cdot \boldsymbol{t}_{s, i}\left(t^{\prime}\right)}{\left|\boldsymbol{t}_{0, i}\left(t^{\prime}\right)\right| \cdot\left|\boldsymbol{t}_{s, i}\left(t^{\prime}\right)\right|}=\left(1+\left(u_{s, i}\left(t^{\prime}\right)-u_{s-1, i}\left(t^{\prime}\right)\right)^{2}\right)^{-\frac{1}{2}} .
$$

Dabei mitteln wir zum Einen über die Zeit, zum Anderen über alle $N_{Y}$ Ketten. Wir definieren demgemäß das diskrete Analogon der Tangens-Korrelationsfunktion im thermischen Mittel:

$$
\begin{aligned}
& C_{t}(s) \equiv\left\langle\hat{\boldsymbol{t}}_{0} \cdot \hat{\boldsymbol{t}}_{s}\right\rangle:=\lim _{t \rightarrow \infty} \frac{1}{t} \int_{0}^{t}\left(\hat{\boldsymbol{t}}_{0} \cdot \hat{\boldsymbol{t}}_{s}\right)\left(s, t^{\prime}\right) \mathrm{d} t^{\prime}, \mathrm{mit} \\
&\left(\hat{\boldsymbol{t}}_{0} \cdot \hat{\boldsymbol{t}}_{s}\right)\left(s, t^{\prime}\right):=\frac{1}{N_{Y}} \cdot \sum_{i=1}^{N_{Y}}\left(\hat{\boldsymbol{t}}_{0, i} \cdot \hat{\boldsymbol{t}}_{s, i}\right)\left(i, s, t^{\prime}\right) .
\end{aligned}
$$

In der Abbildung (2.36) sind die Richtungskorrelationen in Form von $C_{t}(s)$ für frei bewegliche Ketten aufgetragen. Die Ketten können folglich weder durch Crosslinks miteinander in Wechselwirkung treten noch sich durch eine Excluded Volume-Wechselwirkung in ihren thermischen Bewegungen beeinflussen. Alle $N_{Y}$ Ketten sind somit unabhängig voneinander und werden hierbei lediglich zur statistischen Mittelung benötigt.

Man kann beobachten, dass die Richtungskorrelation erwartungsgemäß mit zunehmendem Segmentindex $s$ abfällt, wobei die fluktuierenden Bond-Vektoren bei steiferen Ketten umso stärker in Richtung des starren Referenz-Bond-Vektors $\boldsymbol{t}_{0, i}$ ausgerichtet sind. Dass der Abfall von $C_{t}(s)$ nicht wie in der theoretischen Vorhersage 2.67) exakt einem exponentiellen Verlauf folgt, liegt an einer Einschränkung in unserem Modell: Da 


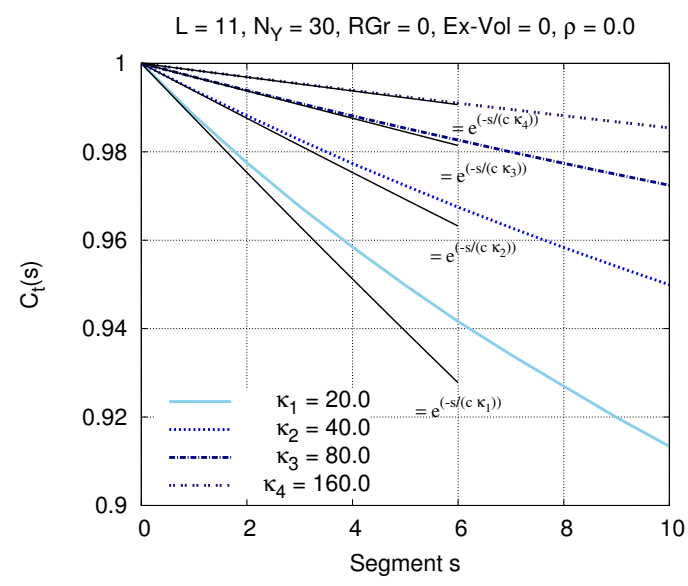

Abbildung 2.36.: $C_{t}(s)$, aufgetragen über bei verschiedenen Werten von $\kappa$ und $\rho=0$

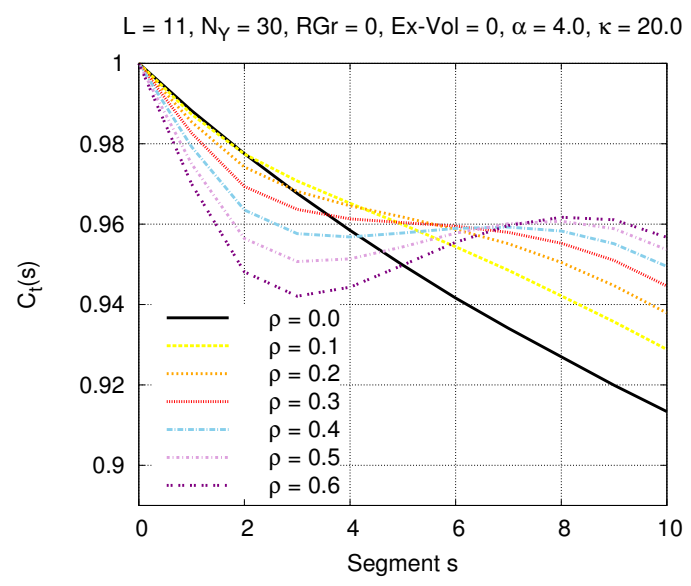

Abbildung 2.37.: $C_{t}(s)$, aufgetragen über $s$ bei verschiedenen Werten von $\rho$

die Ketten sich nicht vollständig willkürlich biegen können, sondern alle Segmentenden stets auf den äquidistanten Gitterpunkten $x_{s}=b \cdot s$ entlang der $x$-Achse zu liegen kommen, kann der höhere Wert von $C_{t}(s)$ gegenüber der theoretischen Vorhersage nachvollzogen werden. Allerdings wird dieser Effekt bzw. diese Abweichung mit wachsendem $\kappa$-Wert und den damit verbundenen kleiner werdenden Segment-Auslenkungen $u_{s, i}\left(t^{\prime}\right)$ wie erwartet immer kleiner.

Wird bei einer konstant gehaltenen Biegesteifigkeit die Anzahl der Crosslinks im System variiert, so kommt es zu deutlichen Abweichungen im Vergleich zum obigen Fall. Wie in Abbildung (2.37) zu sehen ist, führt eine Zunahme der Crosslink-Dichte am festen Ende zu einer geringeren Richtungskorrelation bezüglich $\left.C_{t}(s)\right|_{\rho=0}$, am freien Ende hingegen zum gegenteiligen Effekt, das heißt zu einer Erhöhung von $\left.C_{t}(s)\right|_{\rho>0}$ gegenüber $\left.C_{t}(s)\right|_{\rho=0}$. Dieser Effekt prägt sich mit wachsender Crosslinkzahl umso stärker aus, so dass bei hohen Crosslinkdichten lokale Minima im Funktionsverlauf entstehen.

Erklären lässt sich dies mit der Entstehung von Polymerbündeln, die sich mit wachsendem $\rho$ umso stärker herausbilden. Dies führt dazu, dass sich die beweglichen KettenAbschnitte in der Nähe des festen Endes, das heißt im Übergangsbereich zwischen Substrat und Bündel, stärker krümmen und sich aufgrund der thermischen Bewegungen im Mittel stärker von der axialen, geraden Ausrichtung wegbewegen als im Falle freier Ketten. Damit fällt die Richtungskorrelation bei Ketten, die durch Crosslinks miteinander verknüpft sind, am festen Ende niedriger aus als bei freien Ketten. Im Gegensatz dazu bewirkt die Erhöhung der Crosslink-Dichte eine Verringerung der mittleren Auslenkungen am freien Ende. Dies bedeutet, dass die durch Crosslinks miteinander vernetzten Ketten um die Referenzrichtung geringere Fluktuationsbewegungen ausführen als freie Ketten, was sich am freien Ende durch eine mit $\rho$ wachsenden Richtungskorrelation zeigt.

Wie sich die Richtungskorrelation bei der Aktivierung der Excluded Volume-Wechselwirkung ändert, wird in wenigen Worten im Anhang A.5 erläutert. 


\subsection{Transversale Fluktuationen}

In diesem Kapitel gehen wir auf die transversalen Fluktuationen der beweglichen Polymersegmente ein und zeigen, wie sich die mittlere quadratische Verschiebung (Mean Square Displacement (MSD)) entlang der Konturlänge, das heißt als Funktion von $s$ verhält. Diese wird definiert durch [91,111]

$$
\operatorname{MSD}(s):=\frac{1}{N_{Y}} \cdot \sum_{i=1}^{N_{Y}}\left(\left\langle u_{s, i}^{2}\right\rangle-\left\langle u_{s, i}\right\rangle^{2}\right),
$$

mit den nach Gleichung (2.29) festgelegten thermischen Mittelwerten der Segmentauslenkungen $\left\langle u_{s, i}\right\rangle$ und deren Quadrate $\left\langle u_{s, i}^{2}\right\rangle$. Wir widmen uns zunächst freien Ketten mit verschiedenen Biegesteifigkeiten $\kappa$, die keinen Wechselwirkungen durch andere Ketten unterworfen sind und nur thermische Bewegungen ausführen (siehe Abb. (2.38)). Wir können nachweisen, dass am freien Kettenende, genügend weit von der Substratoberfläche entfernt, die mittlere quadratische Verschiebung exponentiell mit $s$ wächst und umgekehrt proportional zu $\kappa$ ist, das heißt $\operatorname{MSD}(s) \propto s^{3} / \kappa$, in Übereinstimmung mit der theoretischen Vorhersage 6, 14,75. In einer weiteren Messung fügen wir dem

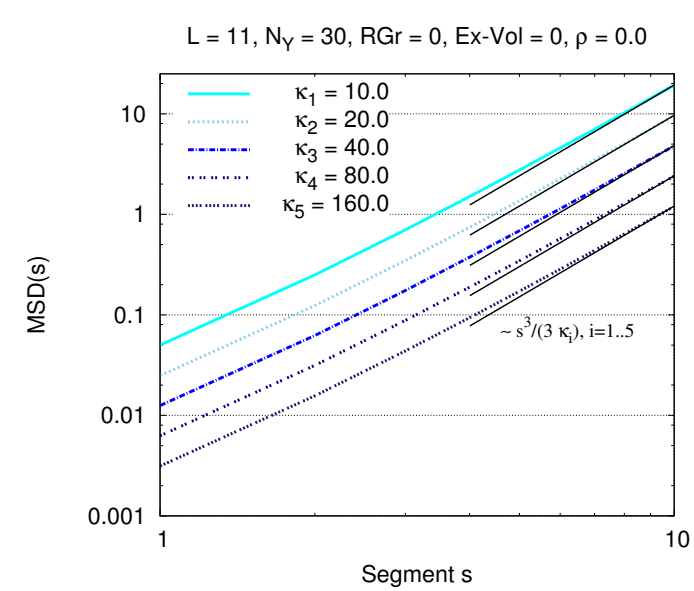

Abbildung 2.38.: $\operatorname{MSD}(s)$ für freie Ketten als Funktion von $s$ für verschiedene $\kappa$-Werte

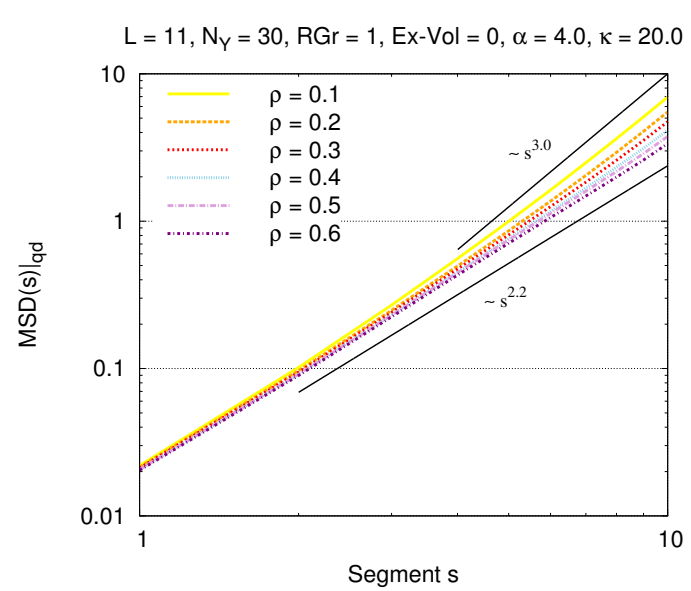

Abbildung 2.39.: $\left.\operatorname{MSD}(s)\right|_{\mathrm{qd}}$ als Funktion von $s$ für verschiedene $\rho$-Werte

System Crosslinks hinzu und betrachten den Quenched Disorder-Mittelwert bezüglich $r_{\max }=20$ voneinander unabhängigen Runs mit $\mathcal{R} G r=1$. Das Ergebnis ist in Abbildung (2.39) aufgetragen. Aufgrund der gegenseitigen Verknüpfungen der Ketten durch Crosslinks hindern sich die Ketten gegenseitig daran, freie thermische Fluktuationsbewegungen auszuführen. Diese Tatsache spiegelt sich in einem geringeren Anstieg von $\operatorname{MSD}(s)$ in Form eines niedrigeren Exponenten bezüglich $s$ wider. So folgt das Mean Square Displacement der durch Crosslinks vernetzten Ketten am festen Ende unabhängig von $\rho$ einem Potenzgesetz, genauer gesagt gilt die nichtlineare Proportionalität $\left.\operatorname{MSD}(s)\right|_{\mathrm{qd}} \propto s^{e}$, mit $e \approx 2.2$. Am losen Ende dagegen gibt es eine crosslinkabhängige Abweichung, das heißt für die Potenzfunktion gilt $\left.\operatorname{MSD}(s)\right|_{\text {qd }} \propto s^{e(\rho)}$, mit einem $\rho$-abhängigen Exponenten. Dies führt zu einer Auffächerung der Kurven in 
dem Sinne, dass eine abnehmende Crosslinkzahl zu einem größeren Exponenten $e(\rho)$ führt. Im Grenzfall crosslinkfreier Ketten und somit wechselwirkungsfreier Ketten gilt $e(\rho=0)=3.0$.

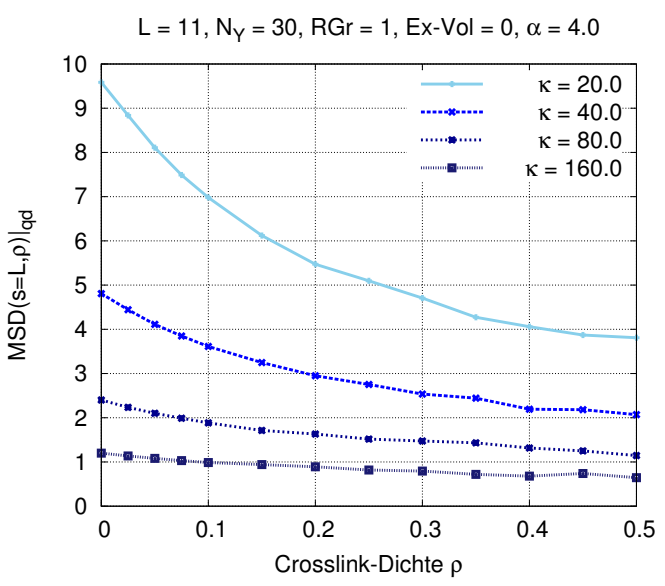

Abbildung 2.40.: $\left.\operatorname{MSD}(s=L, \rho)\right|_{\mathrm{qd}}$ als Funktion von $\rho$ für verschiedene $\kappa$-Werte

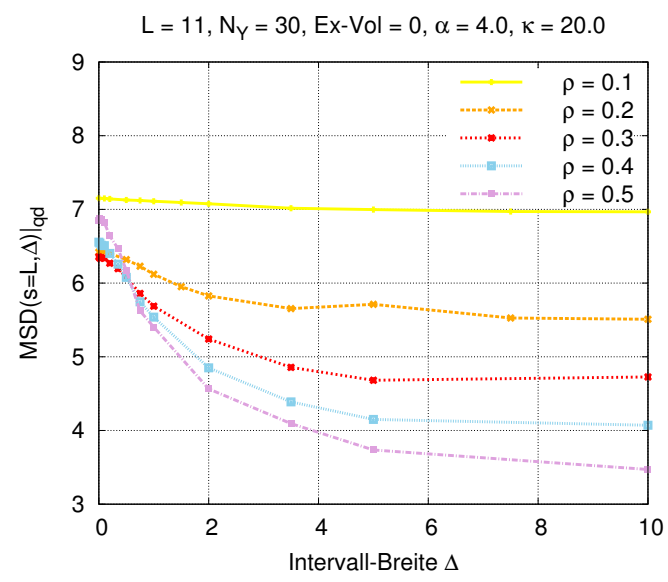

Abbildung 2.41.: $\left.\operatorname{MSD}(s=L, \Delta)\right|_{\mathrm{qd}}$ als Funktion von $\Delta$ für verschiedene $\rho$-Werte

In den folgenden beiden Abbildungen (2.40) und 2.41) wird das Verhalten des Quenched Disorder-Mittels der mittleren quadratischen Verschiebung am Kettenende, das heißt bei festem Segmentindex $(s=L)$ betrachtet. In Abbildung 2.40 wird dabei genauer auf die bereits in Abbildung (2.39) erläuterte Tatsache eingegangen, dass Crosslinks zu einer Verminderung der thermischen Bewegungen führen. Wir sehen, wie die Zunahme der Crosslink-Dichte bei unterschiedlichen Werten der Biegesteifigkeit zu einer nichtlinearen Verringerung der Fluktuationen führt und erkennen direkt, dass das Einbringen von Crosslinks bei steifen Ketten erwartungsgemäß weniger Auswirkungen auf die Fluktuationen hat als dies bei flexiblen Ketten mit niedrigerem $\kappa$-Wert der Fall ist.

Als letzten Punkt in diesem Kapitel wollen wir den Einfluss der Grafting-Ordnung bzw. der Intervallbreite $\Delta$ auf die mittlere quadratische Verschiebung aufzeigen (Abb. (2.41)). Dabei ist es bemerkenswert zu beobachten, dass eine steigende Unordnung der Grafting-Positionen stets mit einer Abnahme der mittleren quadratischen Verschiebung verbunden ist. Dieser Effekt ist umso stärker ausgeprägt, je höher die Crosslink-Dichte ist. Im Grenzfall wechselwirkungsfreier Ketten ist trivialerweise keine $\Delta$-Abhängigkeit vorhanden. Durch die abnehmende Grafting-Ordnung kommt es zu unregelmäßigen Kettenabständen, die das Einfrieren von Bündelstrukturen begünstigen ( Quenched Disorder). Es stellt sich heraus, dass mit wachsender Unregelmäßigkeit der Kettenanordnungen und der daraus folgenden verstärkten Persistenz der Struktur die Ausübung von Fluktuationsbewegungen in wachsendem Maße gehemmt wird. 


\subsection{Winkelverteilungen der Bond-Vektoren}

Wir definieren mit $\theta_{s}$ den Mittelwert des Winkels aller $N_{Y}$ Bond-Vektoren mit SegmentIndex $s, \boldsymbol{t}_{s, i} ; i=1,2, \ldots, N_{Y}$, zur $y$-Achse. Aus der Greenschen Funktion kann für eine Wormlike Chain die Wahrscheinlichkeit abgeleitet werden, das Segment $s$ mit der Segment-Ausrichtung $\theta_{s}$ zu finden, sofern das Segment $s^{\prime}$ im Winkel $\theta_{s^{\prime}}$ ausgerichtet ist [75. Wir übertragen dies auf unseren diskretisierten Fall und betrachten die BondVektoren als das diskrete Analogon der Tangenten-Vektoren (siehe Kapitel 2.19).

Die Greensche Funktion vereinfacht sich wegen $k_{B} T \equiv 1$ und der Grafting-Zwangsbedingung, die die Ausrichtung des ersten Bond-Vektors $\boldsymbol{t}_{0, i}$ durch $\theta_{0}=\frac{\pi}{2}$ festlegt. Sie lautet im zweidimensionalen Fall [75]:

$$
G\left(s, \theta_{s} \mid 0, \pi / 2\right)=\sqrt{\frac{\kappa}{2 \pi s}} \exp \left(-\frac{\kappa\left(\theta_{s}-\frac{\pi}{2}\right)^{2}}{2 s}\right) .
$$

Es liegt offenbar eine gaußförmige Winkelverteilung vor, deren Breite von der Biegesteifigkeit $\kappa$ und dem Segmentindex $s$ abhängt. Im thermischen Mittel erwarten wir entsprechend der analytischen Vorhersage für die Ausrichtung der Bond-Vektoren eine Wahrscheinlichkeitsverteilung $P(s, \theta) \propto G\left(s, \theta_{s} \mid 0, \pi / 2\right)$. In der Abbildung (2.42) sind

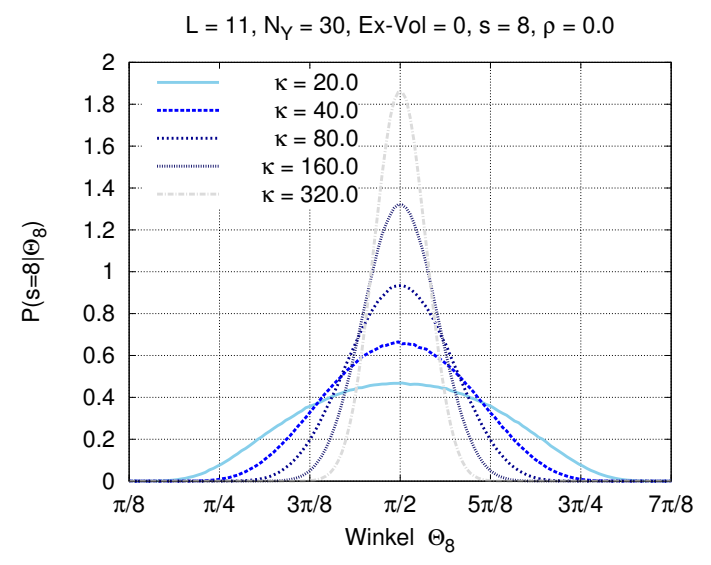

Abbildung 2.42.: $P\left(8, \theta_{8}\right)$ für verschiedene $\kappa$-Werte bei $s=8$

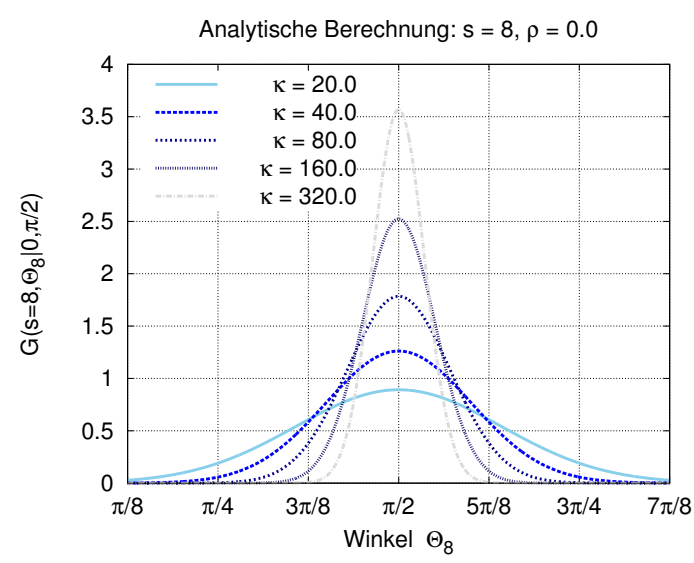

Abbildung 2.43.: $G\left(8, \theta_{8} \mid 0, \pi / 2\right)$ für verschiedene $\kappa$-Werte

für crosslinkfreie Polymerketten mit unterschiedlichen Biegesteifigkeiten die numerisch ermittelten Winkelverteilungen für einen festen Segmentindex $s=8$ aufgetragen. Die Ketten befinden sich im thermischen Gleichgewicht und es wurde über alle voneinander unabhängigen $N_{Y}$ Ketten gemittelt. Daneben zeigt Abbildung (2.43) die dazugehörige analytische Lösung (Gleichung (2.72) ) für den Kontinuumslimes.

Vergleicht man das numerische mit dem analytischen Ergebnis, so ist eine sehr gute Übereinstimmung insbesondere bei hohen $\kappa$-Werten zu erkennen. Lediglich bei relativ biegsamen Ketten (zum Beispiel bei $\kappa=20.0$ ) fällt auf, dass hohe Abweichungen vom Mittelwert $\theta_{8}=\pi / 2$ in der Simulation stärker unterdrückt sind, als die Theorie vorhersagt. Diese kleine Diskrepanz ist allerdings auf die bereits genannte Vereinfachung im 
Modell zurückzuführen, die nur sehr kleine Auslenkungen von der Ruhelage erlaubt.

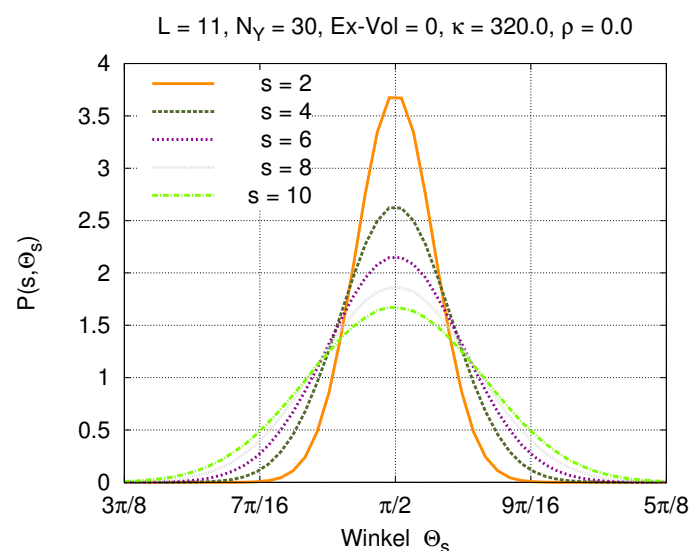

Abbildung 2.44.: $P\left(s, \theta_{s}\right)$ bei festem $\kappa$ für verschiedene Werte von $s$

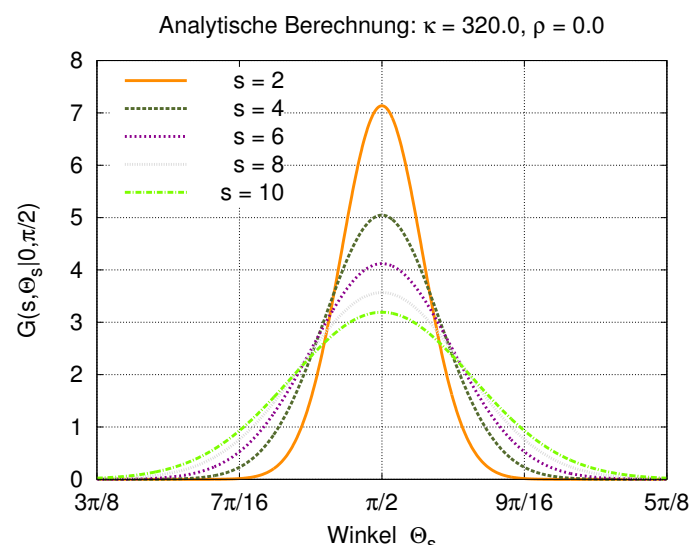

Abbildung 2.45.: $G\left(s, \theta_{s} \mid 0, \pi / 2\right)$ bei festem $\kappa$ für verschiedene Werte von $s$

Die Winkelverteilungen crosslinkfreier Ketten bei unterschiedlichen Gitterpunkten $x_{s}$, aber festem Wert der Biegesteifigkeit (hier $\kappa=320.0$ ), zeigen die Abbildungen (2.44) und (2.45). Sie lassen den Schluss zu, dass insbesondere bei sehr hohen Werten der Biegesteifigkeit die analytisch berechnete Wahrscheinlichkeitsverteilung mit den Simulationsergebnissen sehr gut übereinstimmt. Je höher der Index $s$, desto weiter entfernt befindet sich der Messpunkt von der festen Substratoberfläche und desto breiter wird die Verteilung $P\left(s, \theta_{s}\right)$, im Einklang mit dem Ansteigen der thermischen Schwankungen bzw. der transversalen Fluktuationen hin zum Kettenende.

Natürlich stellt sich auch die Frage, wie sich der Einfluss von Crosslinks auf die Winkelverteilung auswirkt. Dies wird in einem kurzen Kapitel im Anhang A.6 behandelt.

\subsection{Segmentabhängige Crosslink-Dichte $\rho(s)$}

Im Folgenden werden einige charakteristischen Aspekte zur Verteilung der Crosslinks innerhalb des Systems erläutert. Was die Anordnung der Crosslinks betrifft, so gibt es segmentabhängig erhebliche Unterschiede, wie die folgenden Abbildungen verdeutlichen. Wir gehen wieder von äquidistanten Grafting-Points $(\mathcal{R} G r=0)$ und von Ketten ohne Excluded Volume-Wechselwirkung $(E x-\mathrm{Vol}=0)$ aus. Hier wird die bislang pauschal durch den Mittelwert $\rho$ ausgedrückte Parameter-Größe differenzierter, nämlich segmentabhängig betrachtet. Mit der Indikatorfunktion

$$
\mathbb{1}_{\mathcal{C}\left(t^{\prime}\right)}[(s, i)]= \begin{cases}1, & \text { falls }(s, i) \in \mathcal{C}\left(t^{\prime}\right) \\ 0, & \text { sonst }\end{cases}
$$

und der Menge der $R$ schleifenzahlabhängigen Crosslink-Koordinaten

$$
\mathcal{C}\left(t^{\prime}\right)=\left\{\left(s_{1}\left(t^{\prime}\right), i_{1}\left(t^{\prime}\right)\right), \ldots,\left(s_{R}\left(t^{\prime}\right), i_{R}\left(t^{\prime}\right)\right)\right\}
$$


(siehe 2.20) schreiben wir daher

$$
\rho(s) \equiv\left\langle\rho_{s}\right\rangle=\lim _{t \rightarrow \infty} \frac{1}{t} \int_{0}^{t} \rho_{s}\left(t^{\prime}\right) \mathrm{d} t^{\prime} \quad \text { mit } \quad \rho_{s}\left(t^{\prime}\right):=\frac{1}{N_{Y}} \cdot \sum_{i=1}^{N_{Y}} \mathbb{1}_{\mathcal{C}\left(t^{\prime}\right)}[(s, i)] .
$$

Prinzipiell kann beobachtet werden, dass sich die über eine endliche Crosslink-Stärke $\alpha$ verfügenden Crosslinks im thermischen Gleichgewicht bevorzugt in der Umgebung des freien Kettenendes anlagern (siehe Abb. (2.46)). Dieser Effekt tritt mit zunehmender Crosslink-Stärke verstärkt in Erscheinung, das heißt, die Crosslinks sammeln sich mit steigendem $\alpha$ vermehrt am freien Kettenende an. Eine steigende Federstärke unterdrückt ab einem Wert von etwa $\alpha=8.0$ die Anlagerung der Crosslinks in der Nähe der Substratoberfläche, da dies aufgrund der festen Bond-Vektoren $\boldsymbol{t}_{0, i}$ mit einer relativ großen Energieerhöhung verbunden ist, die meist durch den Metropolis-Algorithmus zurückgewiesen wird. Offensichtlich geht die zunehmende Ansammlung von Crosslinks am freien Kettenende mit der verstärkten Bildung von Bündeln einher. Der Zuwachs von $\rho(s)$ wird jedoch mit steigendem $\alpha$ immer schwächer.

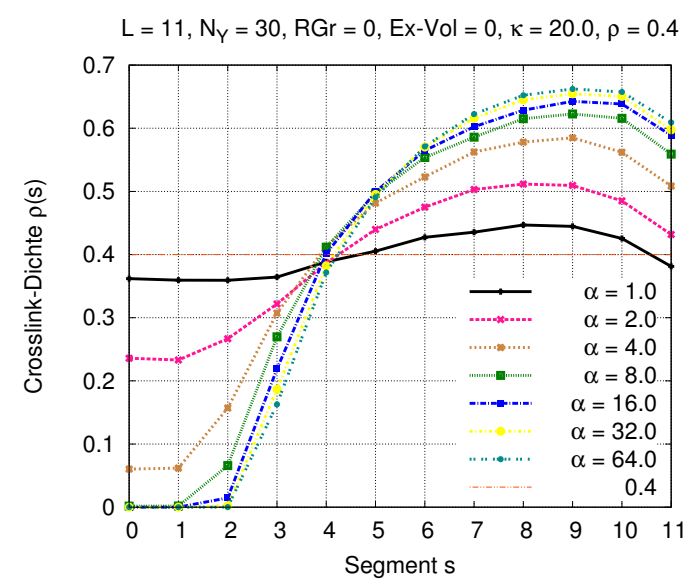

Abbildung 2.46.: Mittlere Crosslink-Dichte $\rho(s)$ als Funktion von $s$ für verschiedene Werte von $\alpha$

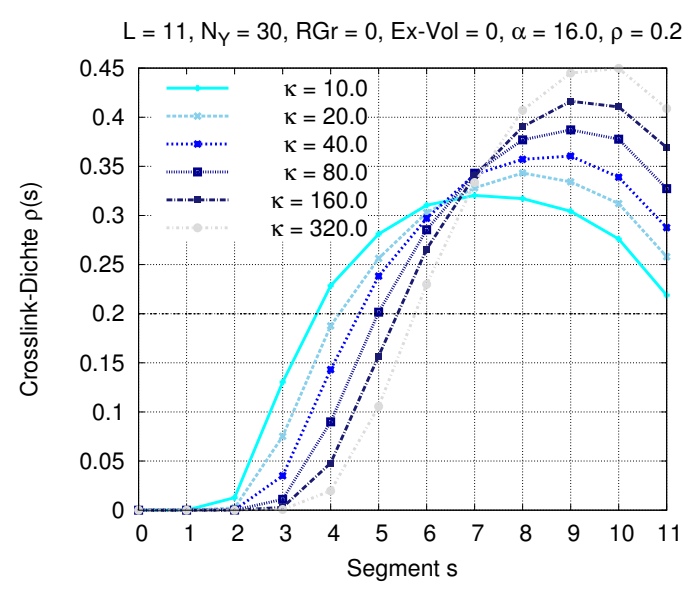

Abbildung 2.47.: Mittlere Crosslink-Dichte $\rho(s)$ als Funktion von $s$ für verschiedene Werte von $\kappa$

Wählt man dagegen einen konstanten, genügend hohen $\alpha$-Wert und variiert die Biegesteifigkeit $\kappa$, so wird deutlich, dass sich die Crosslinks mit steigendem $\kappa$ immer stärker zum freien Kettenende hin verlagern, während das feste Kettenende stets ausgespart bleibt (siehe Abb. (2.47)). Genauer gesagt wächst das Maximum der Crosslinkverteilung und verschiebt sich in die Richtung des freien Kettenendes, während die Breite der Verteilung kleiner wird. Man erkennt hieran, wie die Erhöhung der Biegesteifigkeit und die damit verbundene Verminderung von thermischen Bewegungen das feste Ende kontinuierlich in Richtung des freien Endes erweitern. Im Grenzfall $T=0$ bzw. bei unendlich großem $\kappa$ wären keine Fluktuationsbewegungen mehr möglich und die gesamte Kette wäre unbeweglich wie ein starrer Stab.

Die segmentabhängigen Crosslink-Dichten $\rho(s)$ für unterschiedlich steife Polymerketten und eine unterschiedliche Anzahl an relativ weichen Crosslinks $(\alpha=4.0)$ verdeutlichen 


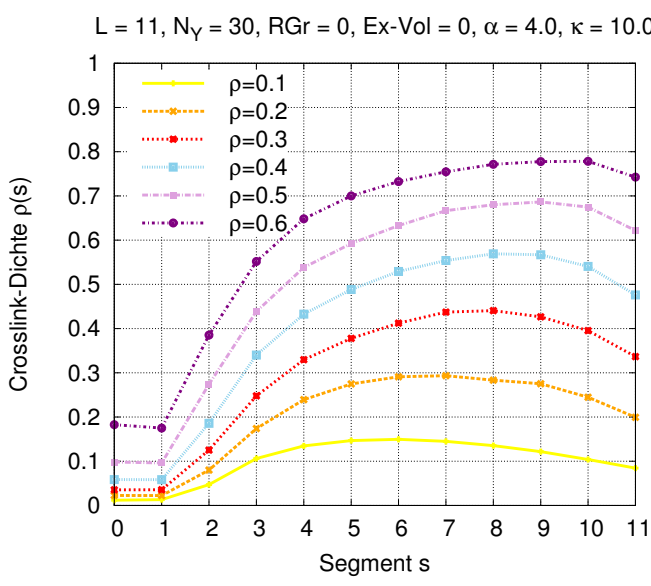

Abbildung 2.48.: Mittlere Crosslink-Dichte $\rho(s)$ als Funktion von $s$ für verschiedene Werte von $\rho$ für $\kappa=10.0$

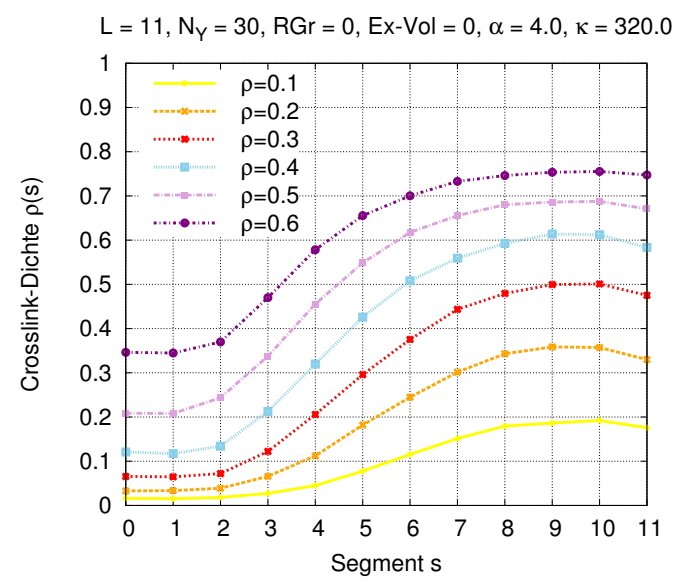

Abbildung 2.49.: Mittlere Crosslink-Dichte $\rho(s)$ als Funktion von $s$ für verschiedene Werte von $\rho$ für $\kappa=320.0$

die beiden nächsten Darstellungen (Abb. 2.48) ) und (Abb. (2.49)). Gerade aufgrund der geringen Federstärke können sich die Crosslinks auch in der Nähe der Substratoberfläche ansiedeln, wobei allerdings generell das lose Ende bevorzugt wird und es somit dort, am losen Ende, zu einer höheren Crosslink-Dichte kommt. Anders als im Falle harter Crosslinks (vergleiche Abbildung (2.47) ) führt hier eine Erhöhung der Biegesteifigkeit zu einer vermehrten Ansiedlung von Crosslinks am festen Kettenende.

\subsection{Mittlere Crosslink-Länge $l_{\mathrm{xl}}$}

Es stellt sich die interessante Frage, wie sich die mittleren Crosslink-Längen der im System befindlichen $R$ Crosslinks im thermischen Gleichgewicht als Funktion der Parameter $\alpha, \kappa$ und $\rho$ verhalten. Diese Größe gibt die zeitliche Mittelung der mittleren Abstände zweier durch Crosslinks verbundenen Segmente an:

$$
l_{\mathrm{xl}}:=\lim _{t \rightarrow \infty} \frac{1}{t} \int_{0}^{t} d\left(t^{\prime}\right) \mathrm{d} t^{\prime} \quad \text { mit } \quad d\left(t^{\prime}\right):=\frac{1}{R} \cdot \sum_{n=1}^{R} d_{s_{n}\left(t^{\prime}\right), i_{n}\left(t^{\prime}\right)} .
$$

Die Größe $d_{s_{n}\left(t^{\prime}\right), i_{n}\left(t^{\prime}\right)}$ bezeichnet die Länge des Crosslinks, der zur Zeit $t^{\prime}$ auf dem Gitterplatz mit den Koordinaten $\left(s_{n}, i_{n}\right)$ platziert ist. Die Abbildungen 2.50 bis 2.52 geben über das Verhalten von $l_{\mathrm{xl}}$ in Abhängigkeit der Parameter $\alpha, \kappa$ und $\rho$ Aufschluss. So wird deutlich, dass erwartungsgemäß härtere Crosslinks mit einer höheren Crosslink-Stärke $\alpha$ miteinander verbundene Ketten stärker zusammenziehen. Diese Annäherung spiegelt sich in kleinen mittleren Crosslink-Längen wider und begünstigt die Bündelbildung.

Durch die Steigerung der Biegesteifigkeit erreicht man einen gegenläufigen Effekt. Gewissermaßen wirken das Federpotential der Crosslinks und die Biegeenergie konträr zueinander. Steifere Ketten leisten der Verbiegung und damit der Kontraktion eines 
Bündels durch die Federkraft der Crosslinks stärkeren Widerstand als flexiblere Ketten mit kleinen $\kappa$-Werten (siehe Abb. 2.50).

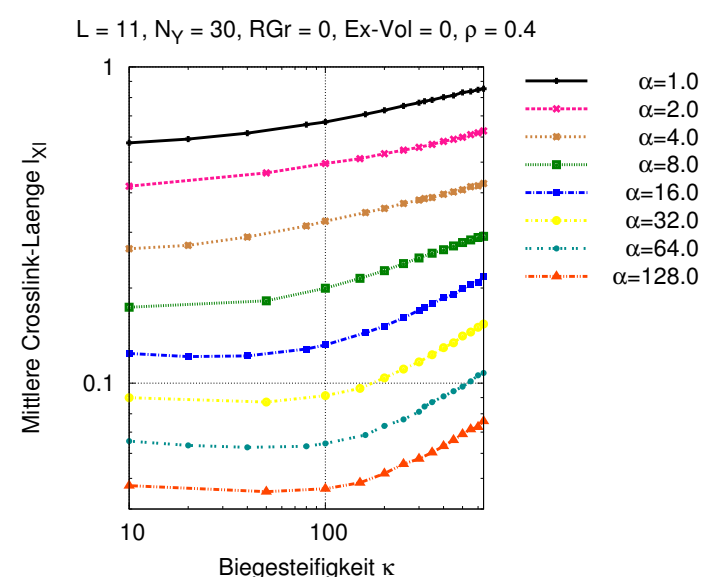

Abbildung 2.50.: Mittlere Crosslink-Länge $l_{\mathrm{xl}}$ als Funktion von $\kappa$ für verschiedene Werte von $\alpha$

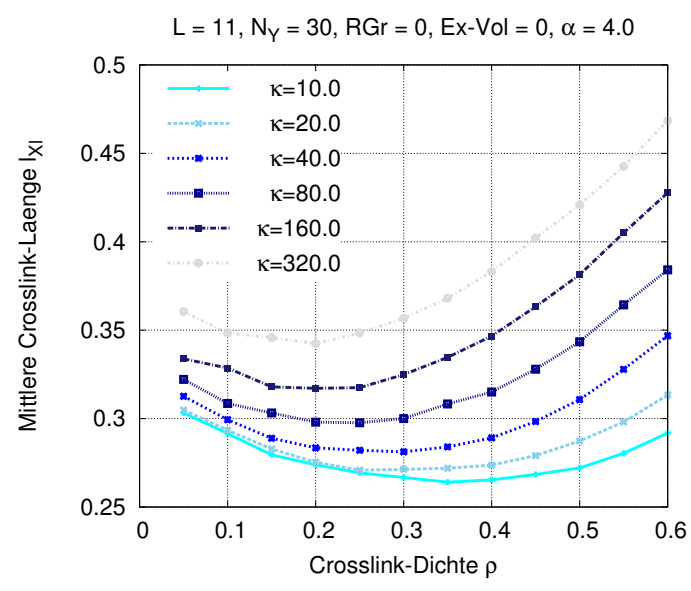

Abbildung 2.51.: Mittlere Crosslink-Länge $l_{\mathrm{xl}}$ als Funktion von $\rho$ für verschiedene Werte von $\kappa$

Eine weitere bemerkenswerte Eigenschaft von $l_{\mathrm{xl}}$ wird in Abbildung (2.51) offensichtlich. Bei festgehaltener, relativ niedriger Crosslink-Stärke wird die Abhängigkeit der mittleren Crosslink-Länge von der Crosslink-Dichte für unterschiedlich steife Ketten präsentiert. Neben der bereits diskutierten Charakteristik, dass höhere Biegesteifigkeiten zu höheren mittleren Crosslink-Längen führen, stellt man für die Variation von $\rho$ kein monotones Verhalten fest. Steigert man die Crosslink-Dichte, von einem sehr niedrigen Wert nahe Null beginnend, zu höheren Werten, so fallen die Werte von $l_{\mathrm{xl}}$ zunächst, durchlaufen ein Minimum und steigen danach wieder monoton mit wachsendem $\rho$ an. Das zu durchlaufende Minimum verschiebt sich mit wachsendem $\kappa$ hin zu niedrigeren Crosslink-Dichten.

Bei niedrigen Crosslink-Dichten überwiegt noch die Minderung der mittleren CrosslinkLänge aufgrund von Bündelbildung. Dieser Effekt wird bei steigender Crosslinkzahl von einem gegenläufigen Effekt überlagert. Der in Bezug auf die Gesamtzahl relative Anteil an Crosslinks in Substratnähe steigt überproportional mit wachsendem $\rho$ (siehe Abbildungen (2.48) und 2.49) in Kapitel 2.22), das heißt, eine wachsende Zahl an Crosslinks kann die Ketten weniger stark zusammenziehen, was dementsprechend zu einer höheren mittleren Crosslink-Länge führt.

$\mathrm{Zu}$ guter Letzt geben wir $l_{\mathrm{xl}}$ als Funktion von $\alpha$ für unterschiedliche Biegesteifigkeiten an (siehe Abb. (2.52)) und erhalten als Ergebnis dabei unmittelbar die Proportionalität zwischen der mittleren Crosslink-Länge und der Crosslink-Stärke: $l_{\mathrm{xl}} \propto \frac{1}{\sqrt{\alpha}}$. 


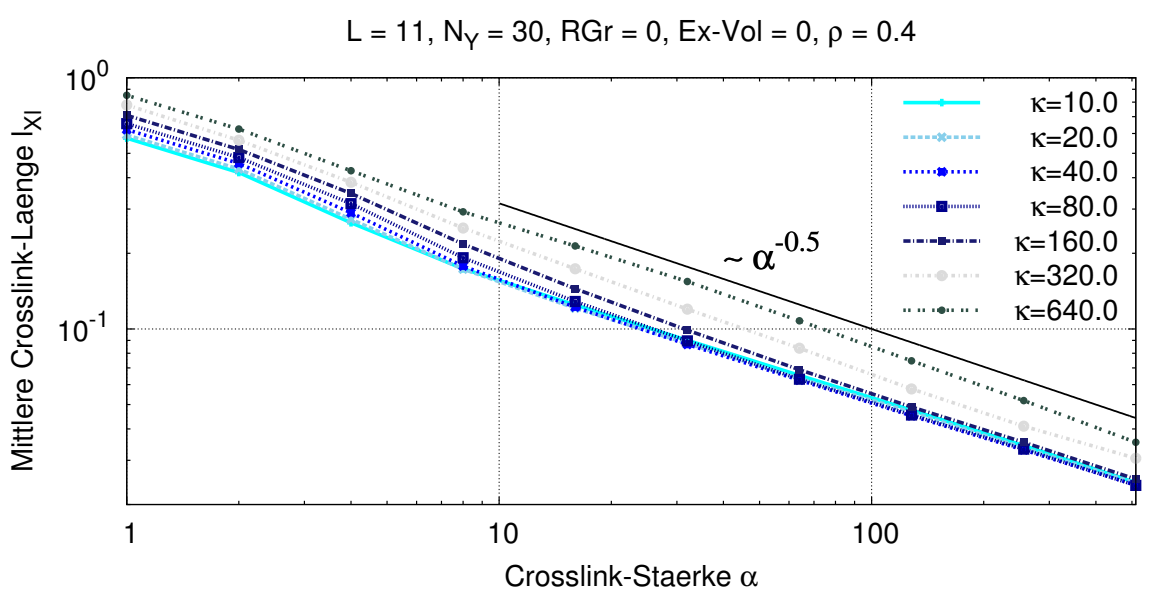

Abbildung 2.52.: Mittlere Crosslink-Länge $l_{\mathrm{xl}}$ als Funktion von $\alpha$ für verschiedene Werte von $\kappa$

\subsection{Zusammenfassung und Ausblick}

In diesem Kapitel haben wir im Rahmen der statistischen Physik mit Hilfe von MonteCarlo-Simulationsmethoden ein System behandelt, das aus semiflexiblen Polymerketten besteht, die senkrecht auf eine Substratoberfläche aufgepfropft sind und über reversible Crosslinks miteinander in Wechselwirkung stehen. Die Motivation zur numerischen Analyse dieses Systems beruht auf dem Bezug zu Biopolymeren im Zytoskelett biologischer Zellen. Solche Polymere wie zum Beispiel Actinfilamente sind semiflexible Polymerketten, die sich mittels actin-bindender Proteine zu hoch geordneten Strukturen anordnen können und so zur Erfüllung wichtiger innerzellulärer Prozesse beitragen $3,4,6,21,31$.

Crosslinks bewirken zwischen parallel ausgerichteten Polymerketten eine Wechselwirkung in Form von kollektiven Phänomenen. Während im crosslinkfreien System alle Ketten unabhängig voneinander Fluktuationsbewegungen ausführen, führt das Hinzufügen von Crosslinks zu Korrelationen zwischen den Segmentpositionen und weiter zur Bildung von Bündelstrukturen. Die Bündelstruktur bildet sich sowohl durch eine steigende Crosslinkzahl als auch durch eine zunehmende Crosslink-Stärke deutlicher heraus, was mit Hilfe des Strukturfaktors verdeutlicht werden kann. Daraus kann abgeleitet werden, dass bei diesem Modell ein kontinuierlicher Phasenübergang vom Zustand ungeordneter Ketten in einen Zustand mit einer geordneten Bündelstruktur existiert.

Die Anordnung der Grafting-Points auf dem Substrat ist von essentieller Bedeutung für die Persistenz der Bündelstruktur. Sie zeigt, dass lediglich äquidistant gegraftete Ketten und damit vollkommene Homogenität der Grafting-Positionen dazu führt, dass keine eingefrorenen Kettenstrukturen enstehen und die Bündel in der Lage sind, entlang der $y$-Achse zu wandern. Im Unterschied dazu führen unregelmäßige Grafting-Konfigurationen bei Ketten, die durch Crosslinks miteinander verknüpft sind, zu einer persistenten Bündelstruktur. Die Stärke dieses Quenched Disorder-Effektes nimmt 
sowohl mit steigender Crosslink-Dichte als auch mit wachsender Grafting-Unordnung $\mathrm{zu}$.

Außerdem konnte nachgewiesen werden, dass eine beliebig gewählte, feste GraftingAnordnung unabhängig von der Startkonfiguration der Segmentauslenkungen und der Crosslink-Anfangsverteilung im Grenzfall $t \rightarrow \infty$ stets zur identischen Bündelstruktur führt (Memory-Effekt) 116.

Für zukünftige Untersuchungen wäre es sicherlich von Interesse, das System um eine Dimension zu erweitern und gegraftete Polymerketten auf einer zweidimensionalen Oberfläche zu betrachten. Damit verbunden würden sich viele weitere Fragen stellen, zum Beispiel:

- Von welcher Ordnung ist der Phasenübergang zwischen einem Zustand ungeordneter Ketten und einem geordneten Bündelzustand?

- Gibt es bei einer zweidimensionalen Substratoberfläche ebenfalls einen Quenched Disorder- oder einen Memory-Effekt?

- Hätte die Variation der Grafting-Ordnung auch entsprechenden Einfluss auf diese Effekte?

Mit der Erweiterung der System-Dimension wäre jedoch eine Vervielfachung der Freiheitsgrade verbunden, was zu deutlich längeren Rechenzeiten führen würde.

Darüber hinaus könnte man untersuchen, welche Phänomene bei der Einführung unterschiedlicher Monomereinheiten zu beobachten sind und welche Unterschiede zum Beispiel bei der numerischen Behandlung von Copolymeren 108 im Vergleich zu den Monomeren auftreten würden. Außerdem könnte versucht werden, unterschiedliche Wechselwirkungspotentiale sowohl für Ketten als auch für Crosslinks in Betracht zu ziehen. Zum Beispiel wäre es möglich, Wechselwirkungen zu berücksichtigen, die über die benachbarten Ketten hinausgehen und von den Ketten selbst anstatt von den Crosslinks ausgehen. Auch andere Charakteristiken der Crosslinks wie zum Beispiel die Möglichkeit, mehr als zwei Ketten miteinander verknüpfen zu können oder eine mögliche Verbindung mit übernächsten Nachbarn, könnten zu zusätzlichen interessanten Phänomenen führen. 


\section{Spannungs-Dehnungs-Kurve von vernetzten Biopolymeren}

\subsection{Einführung}

Durch Crosslinks vernetzte Polymere treten häufig in künstlichen Materialien wie auch in biologischen Systemen auf und prägen deren elastische Eigenschaften in hohem Maße [13, 37]. Als Beispiele hierfür wären nematische Elastomere [114 und durch actinbindende Proteine miteinander verknüpfte Actinfilamente zu nennen [21].

Insbesondere Actinfilamente können sich in einer Vielzahl von unterschiedlichen Strukturen anordnen, wie zum Beispiel in Form von Bündeln oder Fasernetzwerken, um somit zur gewünschten Funktion innerhalb einer Zelle beizutragen [4, 66, 67. Das heißt, die allgemeinen sowie spezifischen mechanischen Eigenschaften einer Zelle sind auf die molekulare Struktur des Zytoskeletts und damit auf seine Komponenten zurückzuführen $[6]$.

In diesem Kapitel betrachten wir mit mathematisch-analytischen Methoden ein Modellsystem, das aus zwei identischen Wormlike Chains (WLCs) 72 , besteht, die im Rahmen der Weakly Bending-Näherung [13,87, beschrieben werden.

Die beiden parallel zueinander ausgerichteten Polymerketten dieses Modellsystems sind durch eine feste Anzahl irreversibler und entlang der Bogenlänge äquidistant angeordneter Crosslinks miteinander verknüpft. Die als harmonische Federn modellierten Crosslinks beeinflussen die elastischen Eigenschaften des Systems derart, dass deren Präsenz die thermischen Fluktuationen minimiert und damit die Biegesteifigkeit des Modellsystems erhöht. Die Ketten sind entlang einer Achse ausgerichtet und werden axial einer Zugbelastung ausgesetzt und auf diese Weise auseinandergezogen bzw. gedehnt.

Unser Modellsystem ist an real existierende Biopolymere angelehnt. Viele Makromoleküle wie die DNS oder F-Actinfilamente als Bestandteil des Zytoskeletts [3, 31] sind durch ihre Semiflexibilität gekennzeichnet und werden durch ihre Biegesteifigkeit charakterisiert 35, 47. Des Weiteren hängt eine Vielzahl von biologischen Prozessen wie zum Beispiel die Muskelkontraktion von den mechanischen Eigenschaften der Actinfilamente ab 24. Zu deren Beschreibung im Rahmen der statistischen Physik muss das für flexible Ketten verwendete Gaußsche Kettenmodell [23,50,64] erweitert werden, was uns zum oben genannten Wormlike Chain-Modell führt.

Zur analytischen Behandlung von doppelsträngigen, miteinander gekoppelten Biopolymeren wurde ein einfaches Modell, das sogenannte Railway-Track-Modell, eingeführt [28]. Es besteht aus zwei Wormlike Chains, die durch harmonische Federn mitein- 
ander gekoppelt sind. Auf diesem Railway-Track-Modell basieren sowohl analytische als auch numerische Studien zur statistischen Mechanik von doppelsträngigen semiflexiblen Polymeren [84].

Das Ziel der hier präsentierten mathematischen Betrachtung besteht in der Berechnung der Spannungs-Dehnungs-Kurve (Force-Extension-Relation) des beschriebenen Modellsystems. Das bedeutet, wir sind an der Relation zwischen der angelegten Zugkraft und dem daraus resultierenden End-zu-End-Abstand interessiert. Es werden hierbei verschiedene Lösungswege vorgestellt, um das analytische Problem zu lösen. Darüber hinaus werden wir auf die Grenzfälle einer unendlichen Crosslink-Stärke (Harte Crosslinks) und einer hohen Zugkraft (Strong-Stretching-Limit) eingehen und sehen, inwieweit sich die Ergebnisse dabei vereinfachen. In einer Veröffentlichung von A. v. d. Heydt, D. Wilkin, P. Benetatos und A. Zippelius [51] werden entsprechende Resultate diskutiert.

Die technischen Fortschritte der letzten Jahrzehnte ermöglichten eine Vielzahl von Experimenten im Bereich der Einzelmolekülmessungen. Dabei wurden mechanische Messungen der Force-Extension und andere Elastizitätsmessungen an Makromolekülen durchgeführt, die sich analytisch mit dem $W L C$-Modell beschreiben lassen, wie zum Beispiel den DNS-Molekülen oder Actin-Filamenten [19,69, 83]. So konnten auch mit Hilfe der Rasterkraftmikroskopie (Atomic Force Microscopy) und optischen Pinzetten (Optical Tweezers) wichtige Beiträge zur Erkundung der Elastizitätseigenschaften, sowohl von einzelsträngiger als auch doppelsträngiger DNS gewonnen werden 20, 24, 105, 113.

In Anlehnung an das Netzwerk des Zytoskeletts wurde ein aus starren Stäben (Rigid Rods) bestehendes Zufallsnetzwerk (Random Network) [117] sowie ein Modellsystem aus durch Crosslinks vernetzten, steifen Filamenten untersucht, um Elastizitätseigenschaften zu studieren [49]. Im Rahmen der statistischen Mechanik waren zudem semiflexible Bündel, die aus Wormlike Chains bestehen, Gegenstand einer analytischen Studie [52]. Des Weiteren sind im Rahmen einer semimikroskopischen Theorie die Formierung von anisotropen Zufallsnetzwerken semiflexibler Polymere untersucht worden [10]. Dabei spielen permanente und zufällig platzierte Crosslinks die entscheidende Rolle hinsichtlich der Netzwerkstruktur. Außerdem wurde der Effekt von permanenten, zufällig gesetzten Crosslinks auf die Struktur und die Elastizität einer Anordnung von ausgerichteten Polymeren, die zwischen zwei Ebenen eingeschlossen sind, studiert [109]. Die Crosslinks werden hierbei als eingefrorene Unordnung (Quenched Disorder) betrachtet.

Elastische Eigenschaften wurden auch an einem Komposit-Netzwerk untersucht, das aus gebündeltem und mittels Crosslinks verknüpftem F-Actin besteht [37]. Dabei wird gezeigt, welch hohen Einfluss sowohl die Variation der Crosslink-Dichte als auch die der Actin-Konzentration auf die Elastizität hat.

Eine theoretische Studie zur entropischen Elastizität von Wormlike Chains in zwei Dimensionen wurde mit Hilfe der Zustandssumme in [97] präsentiert. Die ForceExtension-Relation zweier Weakly Bending WLCs, die mittig durch einen einzelnen Crosslink miteinander verknüpft sind, war in [13] Gegenstand der mathematisch-analytischen Analyse. Darüber hinaus wurde der Einfluss einer bogenlängenabhängigen, intrinsischen Eigen-Krümmung (Spontaneous Curvature) bei Weakly Bending-Filamenten 
auf die Force-Extension analytisch untersucht [11, 12]. Unterschiedliche Randbedingungen an den Kettenenden haben signifikante Auswirkungen auf die transversalen Fluktuationen und die Force-Extension-Relation, was in 98 gezeigt wird.

\subsection{Beschreibung des Modellsystems}

Als Ausgangspunkt der analytischen Berechnung betrachten wir zwei identische, semiflexible Wormlike Chains [72,100, die entlang der $x$-Achse ausgerichtet sind. Ein wichtiges Charakteristikum der Ketten ist ihre Biegesteifigkeit $\kappa$, die mit der Persistenzlänge $L_{P}$ über die Relation

$$
L_{P}=\frac{2 \kappa}{(D-1) k_{B} T}
$$

verbunden ist [47]. Mit $D$ wird die Dimensionalität bezeichnet, die in unserem Falle $D=2$ beträgt. Die Raumkurven der beiden Ketten bzw. die Kettenkonformationen sind nach der Bogenlänge parametrisiert und werden durch den jeweiligen Positionsvektor $\boldsymbol{r}_{j}(s), j \in\{1,2\}$, mit $s \in[0, L]$ beschrieben. Die beiden Kettenenden bei $s=0$ und $s=L$ sind nicht miteinander verbunden, sondern liegen lose aneinander.

Eine weitere wichtige Eigenschaft des zu behandelnden Systems besteht in einer festen Zahl von $N$ Crosslinks, die die beiden Polymere entlang der Konturlänge äquidistant miteinander verknüpfen. Das bedeutet, dass sich die Verknüpfungspunkte $s_{b}$, an denen die Crosslinks mit den Polymersträngen verbunden sind, an den Positionen

$$
s_{b}=\frac{b L}{N+1} ; b=1,2, \ldots, N
$$

befinden. Die Crosslinks werden als harmonische Federn mit einem quadratischen Potentialterm modelliert, mit einer Crosslink-Stärke $g \propto k_{B} T$. Zur Veranschaulichung sind in Abbildung (3.1) schematisch zwei Weakly Bending Wormlike Chains dargestellt, die entlang der $x$-Achse mit der Kraft $f$ gedehnt werden. Die beiden Ketten sind hierbei durch $N=3$ Crosslinks miteinander verbunden.

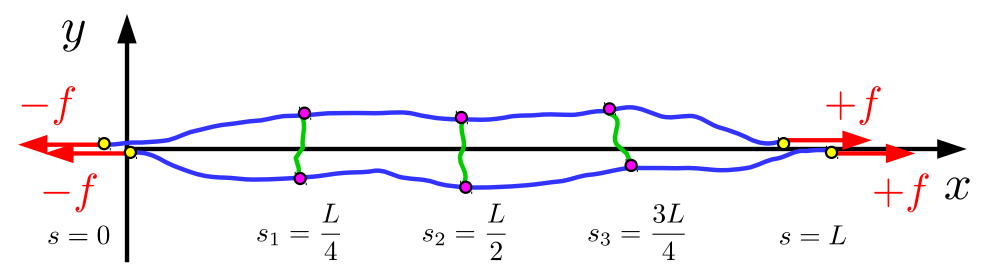

Abbildung 3.1.: Schematische Darstellung zweier WLCs, die durch $N=3$ Crosslinks miteinander verknüpft sind und mit der Kraft $f$ auseinandergezogen werden

Am soeben eingeführten und beschriebenen System wird nun die Abhängigkeit zwischen einer angelegten Kraft und der daraus resultierenden Dehnung analytisch ermittelt. Das heißt, das Ziel besteht in der Bestimmung einer Force-Extension-Relation. 
Die Kraft $f$ wirkt jeweils an den Kettenenden entlang der $x$-Achse, so dass die Polymerketten längs dieser Achse auseinandergezogen werden. Die Energie wird in Einheiten von $k_{\mathrm{B}} T$ angegeben, wobei $k_{B} \equiv 1$ gesetzt wird. Das Energiefunktional bzw. den Hamiltonian dieses Systems geben wir in Abhängigkeit des Tangentenvektors

$$
\boldsymbol{t}_{j}(s)=\frac{\mathrm{d} \boldsymbol{r}_{j}(s)}{\mathrm{d} s}=\left(\frac{\mathrm{d} x_{j}}{\mathrm{~d} s}, \frac{\mathrm{d} \boldsymbol{r}_{j, \perp}}{\mathrm{d} s}\right)
$$

an 13, 64. Hierbei gilt es, die lokale Undehnbarkeitsbedingung (Local Inextensibility Constraint) entlang der Konturlänge zu berücksichtigen. In diesem Sinne gilt dann

$$
\left|\boldsymbol{t}_{j}(s)\right|=\sqrt{\left(\frac{\mathrm{d} x_{j}}{\mathrm{~d} s}\right)^{2}+\left(\frac{\mathrm{d} \boldsymbol{r}_{j, \perp}}{\mathrm{d} s}\right)^{2}}=1 .
$$

Schließlich erhalten wir für den Hamiltonian des Systems 13,64

$$
\begin{aligned}
\mathcal{H}\left[\boldsymbol{t}_{j}(s)\right] & =\underbrace{\frac{\kappa}{2} \sum_{j=1}^{2} \int_{0}^{L} \mathrm{~d} s\left(\frac{\mathrm{d} \hat{\boldsymbol{t}}_{j}(s)}{\mathrm{d} s}\right)^{2}}_{\mathcal{H}_{\text {bend }}} \underbrace{-f \hat{\boldsymbol{x}} \sum_{j=1}^{2} \int_{0}^{L} \hat{\boldsymbol{t}}_{j}(s) \mathrm{d} s}_{\mathcal{H}_{\text {stretch }}} \\
& \underbrace{+\frac{g}{2} \sum_{b=1}^{N}\left(\boldsymbol{r}_{1, \perp}\left(s_{b}\right)-\boldsymbol{r}_{2, \perp}\left(s_{b}\right)\right)^{2}}_{\mathcal{H}_{\text {xlink }}},
\end{aligned}
$$

mit dem Tangenten-Einheitsvektor $\hat{\boldsymbol{t}}(s)$ an der Stelle $s$ und dem Einheitsvektor der $x$-Achse $\hat{\boldsymbol{x}}$.

Der erste Term aus (3.5) $\mathcal{H}_{\text {bend }}$ steht für die elastische Biegeenergie (Bending Elastic Energy) und ist proportional zur Biegesteifigkeit $\kappa$ und damit proportional zur Persistenzlänge der Kette.

Der zweite Term in (3.5) $\mathcal{H}_{\text {stretch }}$ beschreibt das Potential der an beiden Kettenenden wirkenden Zugkraft, während der letzte Ausdruck, eine Summe aus $N$ quadratischen Potentialtermen, seinen Ursprung in den dem System zugehörigen Crosslinks hat, das heißt, mit $\mathcal{H}_{\text {xlink }}$ werden die an den Verknüpfungspunkten $s_{b}$ sitzenden $N$ harmonischen Crosslinks modelliert.

Aus Gründen einer einfacheren analytischen Berechnung besteht ein naheliegender Schritt nun darin, die Wormlike Chains in der Weakly Bending-Näherung zu behandeln. Dabei gehen wir von genügend hohen Zugkräften aus, so dass aufgrund der in $x$-Richtung wirkenden hohen Zugkraft der Tangentenvektor nur leicht von der $x$-Achse wegkippt 13, 51, 64. Diese Annahme können wir unter Berücksichtigung der Zwangsbedingung (3.4) verwenden und schreiben [13, 64]:

$$
\hat{\boldsymbol{x}} \cdot \hat{\boldsymbol{t}}=\frac{d x}{d s}=\sqrt{1-\left(\frac{\mathrm{d} \boldsymbol{r}_{\perp}}{\mathrm{d} s}\right)^{2}} \approx 1-\frac{1}{2}\left(\frac{\mathrm{d} \boldsymbol{r}_{\perp}}{\mathrm{d} s}\right)^{2}, \text { sowie }\left(\frac{\mathrm{d}^{2} \boldsymbol{r}}{\mathrm{d} s^{2}}\right)^{2} \approx\left(\frac{\mathrm{d}^{2} \boldsymbol{r}_{\perp}}{\mathrm{d} s^{2}}\right)^{2}
$$

Setzt man diese beiden Näherungen aus (3.6) in den Hamiltonian (3.5) ein und bezieht mit ein, dass die Kettenauslenkung in $y$-Richtung im zweidimensionalen Fall durch 
$\boldsymbol{r}_{\perp}(s)=y(s)$ gegeben ist, so erhalten wir schließlich das Freie-Energie-Funktional des Systems $\mathcal{H}_{0}$ in der Weakly Bending-Näherung nach J. F. Marko und E. D. Siggia 87 plus den in 3.5 eingeführten Crosslink-Term $\mathcal{H}_{\text {xlink }}$ :

$$
\begin{aligned}
\mathcal{H}\left[y_{j}(s)\right] & =\underbrace{\frac{\kappa}{2} \sum_{j=1}^{2} \int_{0}^{L} d s\left(\frac{\mathrm{d}^{2} y_{j}(s)}{\mathrm{d} s^{2}}\right)^{2}+\frac{f}{2} \sum_{j=1}^{2} \int_{0}^{L} d s\left(\frac{\mathrm{d} y_{j}(s)}{\mathrm{d} s}\right)^{2}-2 f L}_{\mathcal{H}_{0}} \\
& +\underbrace{\frac{g}{2} \sum_{b=1}^{N}\left(y_{1}\left(s_{b}\right)-y_{2}\left(s_{b}\right)\right)^{2}}_{\mathcal{H}_{\text {xlink }}} .
\end{aligned}
$$

In den weiteren Rechnungen lassen wir den konstanten Term $-2 f L$ in $\mathcal{H}$ weg.

Zur Lösung dieses Problems verwenden wir Hinged-Hinged-Randbedingungen [13 98 . Das heißt, die Polymerenden werden nicht ausgelenkt, sondern liegen auf der $x$-Achse. Außerdem wirken an diesen Enden keine Drehmomente, das heißt, es gilt

$$
y_{j}(0)=y_{j}(L)=\frac{\mathrm{d}^{2} y_{j}(0)}{\mathrm{d} s^{2}}=\frac{\mathrm{d}^{2} y_{j}(L)}{\mathrm{d} s^{2}}=0, \text { für } j \in\{1,2\} .
$$

Diese Randbedingungen sind experimentell zum Beispiel mit Hilfe der Laser-TweezersTechnik realisierbar 1, 20, 105, 113]. Dabei sind die Polymerenden an Kügelchen befestigt, die sich in optischen Fallen befinden. Auf diese Weise kann an den Enden eine Kraft ohne ein Drehmoment übertragen werden 98 .

Die Randbedingungen (3.8) erlauben es uns, $y_{j}(s)$ in Fourier-Reihen zu entwickeln. Mit der Wellenzahl $q_{l}=\frac{l \pi}{L}$ und der Modenzahl $l \in \mathbb{N}$ (mit der Menge der natürlichen Zahlen $\mathbb{N}$ ) schreiben wir für die Eigenfunktionen 13,86

$$
y_{1}(s)=\sum_{l=1}^{2 M \tilde{n}} J_{l} \sin \left(q_{l} s\right) \quad, \quad y_{2}(s)=\sum_{l=1}^{2 M \tilde{n}} K_{l} \sin \left(q_{l} s\right),
$$

mit $\tilde{n}:=(N+1)$. Die obere Grenze der Modenzahl $2 M \tilde{n}$ mit $M \in \mathbb{N}$ kann theoretisch im Falle unendlich scharfer Auflösung gegen unendlich gehen $(M \rightarrow \infty)$, in der Praxis jedoch ist die Auflösung auf die Größenordnung molekularer Distanzen beschränkt [51]. Wie im weiteren Verlauf zu sehen sein wird, erweist sich die Angabe der Modenzahl als

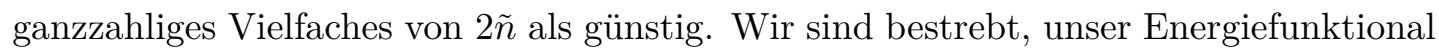
(3.7) in Matrixschreibweise zu formulieren, um das Problem mit Hilfe einer geeigneten Darstellung lösen zu können 13,51. Aus diesem Grunde führen wir den Vektor

$$
\Gamma:=\left(\Gamma_{1}, \Gamma_{2}, \Gamma_{3}, \Gamma_{4}, \ldots\right)=\left(J_{1}, K_{1}, J_{2}, K_{2}, \ldots\right)^{T} \in \mathbb{R}^{4 M \tilde{n}}
$$

ein und können unter Verwendung von Normalmoden den Hamiltonian $\mathcal{H}$ schließlich als quadratische Form darstellen 13,51:

$$
\mathcal{H}[\boldsymbol{\Gamma}]=\frac{1}{2} \boldsymbol{\Gamma}^{T} G \boldsymbol{\Gamma}=\frac{1}{2} \sum_{l, m=1}^{4 M \tilde{n}} \Gamma_{l} g_{l m} \Gamma_{m}, g_{l m}=c_{l m}+p_{l m}
$$


mit den Matrixelementen $g_{l m}, c_{l m}$ und $p_{l m}$ der Matrizen $G, C$ und $P:=U U^{T}$ (siehe unten) und der Menge der reellen $n$-Tupel $\mathbb{R}^{n}$. Man beachte die Ähnlichkeit der Notation zwischen der Crosslink-Stärke $g$ und einem Matrixelement $g_{l m}$ der Matrix $G$. Wir bezeichnen mit $\mathcal{M}(m \times n, \mathbb{R})$ die Menge der reellen $(m \times n)$-Matrizen [30]. Die $4 M \tilde{n} \times 4 M \tilde{n}$-Matrix

$$
G=C+U U^{T}
$$

in (3.11) ist symmetrisch $\left(G=G^{T}\right)$ und positiv definit. Das heißt, für die quadratische Form gilt: $\boldsymbol{\Gamma}^{T} G \boldsymbol{\Gamma}=\sum_{l, m=1}^{4 M \tilde{n}} \Gamma_{l} g_{l m} \Gamma_{m}>0$. Wie in 3.12 verdeutlicht, setzt sich $G$ aus zwei Termen zusammen. Die Diagonalmatrix $C$ ergibt sich aus dem Hamiltonian des Systems ohne Crosslinks $\mathcal{H}_{0}$ und lautet

$$
C=\underbrace{\operatorname{diag}\left(c_{1}, c_{2}, \ldots\right)}_{=: \tilde{C}} \otimes \mathbb{1}_{2}, c_{m m} \equiv c_{m}:=\frac{L}{2} q_{m}^{2}\left(\kappa q_{m}^{2}+f\right) .
$$

Dabei bezeichnet $\otimes$ das Kronecker-Produkt und $\mathbb{1}_{2}$ die $2 \times 2$-Einheitsmatrix.

Die Matrix

$$
P:=U U^{T}=\left(\tilde{U} \otimes\left(\begin{array}{c}
1 \\
-1
\end{array}\right)\right) \cdot\left(\tilde{U}^{T} \otimes(1,-1)\right)=\tilde{U} \tilde{U}^{T} \otimes\left(\begin{array}{cc}
1 & -1 \\
-1 & 1
\end{array}\right)
$$

stammt vom Anteil der $N$ Crosslinks und ist das Matrixprodukt von $U$ mit seiner Transponierten $U^{T} \in \mathcal{M}(N \times 4 M \tilde{n}, \mathbb{R})$. Die Matrixelemente von $\tilde{U}$ sind

$$
\tilde{u}_{l b}=\sqrt{g} \cdot \sin \left(\frac{l b \pi}{\tilde{n}}\right) ; l=1,2, \ldots, 2 M \tilde{n} ; b=1,2, \ldots, N .
$$

Damit ergeben sich unmittelbar die Matrixelemente der $2 M \tilde{n} \times 2 M \tilde{n}$-Matrix $\tilde{P} \equiv \tilde{U} \tilde{U}^{T}$ und wir können schreiben

$$
\tilde{p}_{l m}=g \cdot \sum_{b=1}^{N} \sin \left(\frac{b l \pi}{\tilde{n}}\right) \sin \left(\frac{b m \pi}{\tilde{n}}\right) ; l, m=1,2, \ldots, 2 M \tilde{n} .
$$

Die im Mittelpunkt unseres Interesses stehende physikalische Größe ist die bereits erwähnte Force-Extension-Relation. Das heißt, es wird die Dehnung einer Polymerkette bei Anliegen der Zugkraft $f$ untersucht. Die Force-Extension in der Weakly BendingApproximation errechnet sich aus den thermischen Fluktuationen transversal zur Ausrichtungsrichtung und lautet für eine Kette [13, 87:

$$
\langle x(L)\rangle:=\left\langle x_{1}(L)-x_{1}(0)\right\rangle=L-\frac{1}{2} \int_{0}^{L} d s\left\langle\left(\frac{\mathrm{d} y_{1}(s)}{\mathrm{d} s}\right)^{2}\right\rangle=L-\frac{L}{4} \sum_{l=1}^{\infty} q_{l}^{2}\left\langle J_{l}^{2}\right\rangle .
$$

Aus mathematischen Gründen ist es in (3.17) erlaubt, die endliche Partialsumme wegen $M \rightarrow \infty$ zu einer (konvergenten) Reihe zu erweitern. Mit $\langle\ldots\rangle$ wird der durch den Boltzmann-Faktor $\exp (-\beta \mathcal{H}), \beta=\left(k_{B} T\right)^{-1}$ gewichtete thermische Mittelwert bezeichnet. Hieraus folgt unmittelbar die Notwendigkeit, die Größe

$$
\left\langle J_{l}^{2}\right\rangle=\left\langle\Gamma_{2 l-1}^{2}\right\rangle=g_{2 l-1,2 l-1}^{-1} ; l=1,2, \ldots, 2 M \tilde{n}
$$

zu berechnen, mit anderen Worten, wir benötigen die Diagonalelemente der Inversen von $G$. Die Relation in (3.18) ergibt sich aus der Berechnung von Gaußschen Integralen (siehe Anhang B.1). 


\subsection{Force-Extension mit einem Crosslink $(N=1)$}

Nun können wir unser soeben eingeführtes Modell anwenden, um den Fall mit einem Crosslink $(N=1)$ zu diskutieren. (Die Force-Extension dieses Falls wurde von P. Benetatos, S. Ulrich und A. Zippelius in 13 berechnet.) Dieser eine Crosslink verbindet gemäß 3.2 die beiden Ketten mittig bei $s_{1}=\frac{L}{2}$. Unter Verwendung der mit den Hinged-Hinged-Randbedingungen im Einklang stehenden Eigenfunktionen (3.9) erhalten wir unter Ausnutzung von Orthogonalitätsrelationen (siehe Anhang B.2) für den Hamiltonoperator (3.7)

$$
\begin{aligned}
\mathcal{H}\left[\left\{J_{l}, K_{l}\right\}\right] & =\frac{L}{4} \sum_{l=1}^{4 M}\left[\left(\kappa q_{l}^{4}+f q_{l}^{2}\right)\left(J_{l}^{2}+K_{l}^{2}\right)\right] \\
& +\frac{g}{2} \sum_{l, m=1}^{4 M}\left(J_{l} J_{m}+K_{l} K_{m}-2 J_{l} K_{m}\right) \sin \left(\frac{l \pi}{2}\right) \sin \left(\frac{m \pi}{2}\right) .
\end{aligned}
$$

Bei lediglich einem Crosslink kann die Matrix $U$ als ein gewöhnlicher Vektor $u$ aufgefasst werden (vergleiche $(3.15)$ ) und es gilt:

$$
u=\sqrt{g} \cdot\left(\sin \left(\frac{\pi}{2}\right), \sin (\pi), \ldots\right)^{T} \otimes\left(\begin{array}{c}
1 \\
-1
\end{array}\right) .
$$

Dadurch vereinfacht sich die Berechnung der Inversen von $G$, die mit Hilfe der ShermanMorrison-Formel hergeleitet werden kann [13, 103, 104]:

$$
G^{-1}=\left(C+u u^{T}\right)^{-1}=C^{-1}-\frac{C^{-1} u u^{T} C^{-1}}{1+u^{T} C^{-1} u},
$$

mit der Größe $\lambda:=1+u^{T} C^{-1} u$. Bei $u u^{T}=u \otimes u^{T}$ handelt es sich um das dyadische Produkt des Vektors $u$ mit seiner Transponierten, so dass $u u^{T}$ wieder eine $8 M \times 8 M$ Matrix ergibt.

Wir führen die folgenden dimensionslosen Einheiten ein

$$
z:=\frac{f L^{2}}{\kappa} \quad, \quad \tilde{g}:=\frac{g L^{2}}{T} \quad, \quad \tilde{T}:=\frac{T L}{\kappa}
$$

lassen $M$ gegen Unendlich gehen und erhalten damit für die Größe $\lambda(z)$ :

$$
\begin{aligned}
\lambda(z) & =1+u^{T} C^{-1} u=1+\sum_{l, m=1}^{\infty} c_{l m}^{-1} u_{l} u_{m}=1+\sum_{l=1}^{\infty} c_{l l}^{-1} u_{l} u_{l} \\
& =1+4 \tilde{g} \tilde{T} \sum_{l=1}^{\infty} \frac{\sin ^{2}\left(\frac{l \pi}{2}\right)}{(l \pi)^{2}\left((l \pi)^{2}+z\right)}=1+\frac{\tilde{g} \tilde{T}}{2 z}\left(1-\frac{2}{\sqrt{z}} \tanh \left(\frac{\sqrt{z}}{2}\right)\right)
\end{aligned}
$$

mit den Matrixelementen $c_{l m}^{-1}$ der Inversen von $C$ (siehe Definition in 3.13 ). Folglich 
können wir für die exakte Force-Extension-Relation schreiben:

$$
\begin{aligned}
\frac{\langle x(L)\rangle}{L} & =1-\frac{1}{4} \sum_{l=1}^{\infty} q_{l}^{2}\left\langle J_{l}^{2}\right\rangle=1-\frac{\tilde{T}}{2} \sum_{l=1}^{\infty} \frac{1}{(l \pi)^{2}+z}+\frac{\tilde{g} \tilde{T}^{2}}{\lambda(z)} \sum_{l=1}^{\infty} \frac{\sin ^{2}\left(\frac{l \pi}{2}\right)}{(l \pi)^{2}\left((l \pi)^{2}+z\right)^{2}} \\
& =\underbrace{1-\frac{\tilde{T}}{4 z}(\sqrt{z} \operatorname{coth}(\sqrt{z})-1)}_{\left.\langle x(L)\rangle \cdot L^{-1}\right|_{\mathrm{MS}}}+\underbrace{\frac{\tilde{g} \tilde{T}^{2}}{8 z^{2} \lambda(z)}\left(1-\frac{3}{\sqrt{z}} \tanh \left(\frac{\sqrt{z}}{2}\right)+\frac{1}{2} \operatorname{sech}^{2}\left(\frac{\sqrt{z}}{2}\right)\right)}_{\left.\langle x(L)\rangle \cdot L^{-1}\right|_{\text {xlink }}} .
\end{aligned}
$$

Das Ergebnis in (3.24) setzt sich aus zwei Beiträgen zusammen. Der erste Term $\left(\left.\langle x(L)\rangle \cdot L^{-1}\right|_{\mathrm{MS}}\right)$ rührt vom crosslinkunabhängigen Anteil des Hamiltonians $\mathcal{H}_{0}$ her und entspricht demzufolge dem Marko-Siggia-Term, der das Verhalten einer der beiden Polymerketten ohne Crosslinks wiedergibt 13,87 . Der zweite Term $\left(\left.\langle x(L)\rangle \cdot L^{-1}\right|_{\text {xlink }}\right)$ beschreibt den Crosslink-Anteil an der Force-Extension durch einen einzelnen, bei $s_{1}=L / 2$ fixierten Crosslink mit der Crosslink-Stärke $g$ [13].

Sieht man sich den Kurvenverlauf der Force-Extension als Funktion der Kraft $z$ an (vergleiche Abbildung (3.2) in Kapitel 3.6), so wird deutlich, dass die Steifigkeit des Systems mit Crosslink im Vergleich zum crosslinkfreien Fall erhöht ist. Allerdings wird dieser Unterschied mit zunehmender Kraft geringer. Des Weiteren ist mit der Erhöhung der Crosslink-Stärke $\tilde{g}$ ein nichtlinearer Anstieg der Steifigkeit verbunden.

\section{Harte Crosslinks}

Sehen wir uns nun an, was passiert, wenn die Crosslink-Stärke $\tilde{g}$ unendlich groß wird. Dieser Grenzfall vereinfacht das Ergebnis des Crosslink-Anteils $\left(\left.\langle x(L)\rangle \cdot L^{-1}\right|_{\text {xlink }}\right)$ in (3.24) und wir erhalten für $\tilde{g} \rightarrow \infty$

$$
\frac{\langle x(L)\rangle}{L} \mid \begin{aligned}
& \tilde{g} \rightarrow \infty \\
& \text { xlink }
\end{aligned}=\left(\frac{\tilde{T}}{8 z}\right) \cdot \frac{2 \sqrt{z} \cosh \left(\frac{\sqrt{z}}{2}\right)-6 \sinh \left(\frac{\sqrt{z}}{2}\right)+\sqrt{z} \operatorname{sech}\left(\frac{\sqrt{z}}{2}\right)}{\sqrt{z} \cosh \left(\frac{\sqrt{z}}{2}\right)-2 \sinh \left(\frac{\sqrt{z}}{2}\right)} .
$$

\subsection{Berechnung der Force-Extension für mehrere Crosslinks über die Zustandssumme $\mathcal{Z}$}

Mit Hilfe der Sherman-Morrison-Woodbury-Formel [42,103,104] ist es prinzipiell möglich, die Force-Extension auch für ein System mit mehreren Crosslinks auszurechnen. Im Falle von $N$ Crosslinks erhalten wir für die Inverse von $G$ mit der in (3.14) eingeführten Matrix $U$ (vergleiche (3.21)):

$$
G^{-1}=\left(C+U U^{T}\right)^{-1}=C^{-1}-C^{-1} U\left(\mathbb{1}_{N \times N}+U^{T} C^{-1} U\right)^{-1} U^{T} C^{-1},
$$

mit der $N \times N$-Einheitsmatrix $\mathbb{1}_{N \times N}$. Die Komplexität der zu invertierenden $N \times N$ Matrix $\Lambda:=\left(\mathbb{1}_{N \times N}+U^{T} C^{-1} U\right)$ erhöht sich jedoch mit steigender Crosslinkzahl sehr 
schnell. Daher erscheint es vorteilhaft, zur Berechnung einen alternativen Lösungsweg zu finden. Eine Lösungsmöglichkeit führt über die Berechnung der kanonischen Zustandssumme des Systems [97.

In Bezug auf die Berechnung der Force-Extension-Relation mit Hilfe der Zustandssumme siehe auch die Publikation von A. v. d. Heydt, D. Wilkin, P. Benetatos und A. Zippelius [51].

Die kanonische Zustandssumme eines Systems, das durch den Hamiltonian $\mathcal{H}\left[y_{j}(s)\right]$ beschrieben wird, lautet mit $\beta:=\left(k_{B} T\right)^{-1}$ :

$$
\mathcal{Z}=\int \mathcal{D}[y(s)] \mathrm{e}^{-\beta \mathcal{H}\left[y_{j}(s)\right]} .
$$

In unserem Fall beschreibt das Freie-Energie-Funktional (3.7) zwei Weakly Bending $W L C s$, die durch $N$ Crosslinks äquidistant miteinander verbunden sind und die durch eine Kraft $f$ entlang der $x$-Achse auseinandergezogen werden.

Mittels der Zustandssumme können wir die gewünschte Force-Extension bestimmen, nämlich über die Ableitung des Logarithmus von $\mathcal{Z}$ nach der Kraft $f$ [97:

$$
\langle x(L)\rangle=\left(\frac{k_{B} T}{2}\right) \cdot \frac{\partial \log \mathcal{Z}}{\partial f} .
$$

Da die Force-Extension-Relation für das System ohne Crosslinks bekannt ist [87], können wir uns darauf konzentrieren, lediglich den crosslinkabhängigen Anteil der ForceExtension zu kalkulieren. Das bedeutet, wir berechnen dafür ausschließlich das Verhältnis der Zustandssumme $\mathcal{Z}_{0}$ des crosslinkfreien Systems zur Zustandssumme $\mathcal{Z}$ des gesamten Systems inklusive der $N$ Crosslinks. Für dieses Verhältnis $\mathcal{Z}_{\text {rel }}$ gilt mit Hilfe der Relation für eine quadratische $n \times n$-Matrix $W 5$

$$
(\operatorname{det} W)^{-\frac{1}{2}}=\mathrm{e}^{-\frac{1}{2} \operatorname{Sp} \log W},
$$

sowie mit $\operatorname{det} C \neq 0$ und unter Berücksichtigung von (3.27)

$$
\mathcal{Z}_{\text {rel }}:=\frac{\mathcal{Z}}{\mathcal{Z}_{0}}=\frac{\int \mathcal{D}[y(s)] \mathrm{e}^{-\beta \mathcal{H}[y(s)]}}{\int \mathcal{D}[y(s)] \mathrm{e}^{-\beta \mathcal{H}_{0}[y(s)]}}=\frac{(\operatorname{det} G)^{-\frac{1}{2}}}{(\operatorname{det} C)^{-\frac{1}{2}}}=\left(\operatorname{det}\left(\mathbb{1}+C^{-1} U U^{T}\right)\right)^{-\frac{1}{2}}
$$

Mit Hilfe der Taylorentwicklung des Logarithmus können wir (3.30) umschreiben und finden

$$
\mathcal{Z}_{\text {rel }}=\exp \left\{-\frac{1}{2} \sum_{k=1}^{\infty}\left(\frac{(-1)^{k+1}}{k} \operatorname{Sp}\left(C^{-1} U U^{T}\right)^{k}\right)\right\} .
$$

Die zur Bestimmung von $\mathcal{Z}_{\text {rel }}$ notwendige Berechnung von $\operatorname{Sp}\left(C^{-1} U U^{T}\right)^{k}$ wird im folgenden Kapitel 3.5 gezeigt.

\subsection{Berechnung der Spur von $\log \left(\mathbb{1}+C^{-1} U U^{T}\right)$ :}

Zur Auswertung der Spur von $\left(C^{-1} U U^{T}\right)^{k} ; k=1,2, \ldots, \infty$ aus 3.31 liegt es nahe, die Matrixelemente von $\tilde{P}:=\tilde{U} \tilde{U}^{T}$ aus 3.16 zu ermitteln. Wir finden für diese (siehe 
Anhang B.3

$$
\tilde{p}_{m n}=\left(\frac{g \tilde{n}}{2}\right) \cdot\left(\delta_{m-n, 2 \tilde{n} \mathbb{Z}}-\delta_{m+n, 2 \tilde{n} \mathbb{Z}}\right)
$$

mit der Menge der ganzen Zahlen $\mathbb{Z}$.

Mit der Anzahl der Crosslinks $N$ ist die Periodizität von $\tilde{P}$ verknüpft; demnach ist $\tilde{P}$ periodisch in den Matrixindizes $m$ und $n$ mit der Periode $2 \tilde{n}=2(N+1)$, (siehe Anhang B.3.

Unter Anwendung von Eigenschaften des Kronecker-Produktes [106, 110] (siehe Anhang B.4 sind wir in der Lage, das Matrixprodukt von $C^{-1}$ mit $U U^{T}$ in der folgenden Weise anzugeben:

$$
C^{-1} U U^{T}=\tilde{C}^{-1} \tilde{U} \tilde{U}^{T} \otimes\left(\begin{array}{cc}
1 & -1 \\
-1 & 1
\end{array}\right),
$$

um schließlich die zu berechnende Spur wie folgt zu schreiben:

$$
\operatorname{Sp}\left(C^{-1} U U^{T}\right)^{k}=: \operatorname{Sp}\left((\tilde{A} \otimes F)^{k}\right)=\operatorname{Sp}\left(\tilde{A}^{k}\right) \cdot \operatorname{Sp}\left(F^{k}\right),
$$

mit den beiden Matrizen

$$
\tilde{A}:=\tilde{C}^{-1} \tilde{U} \tilde{U}^{T} \quad \text { und } \quad F:=\left(\begin{array}{cc}
1 & -1 \\
-1 & 1
\end{array}\right)
$$

Damit haben wir die Bestimmung der Spur (3.34) aufgeteilt und wir können getrennt $\operatorname{Sp}\left(\tilde{A}^{k}\right)$ und $\operatorname{Sp}\left(F^{k}\right)$ ermitteln. Es ist $\operatorname{Sp}(F)=2$, die Eigenwerte von $F$ sind $\lambda_{1}=2$ und $\lambda_{2}=0$. Mit den Eigenwerten $\lambda_{1}, \lambda_{2}, \ldots, \lambda_{n}$ einer $n \times n$-Matrix $F$ gilt:

$$
\operatorname{Sp}(F)=\sum_{i=1}^{n} \lambda_{i}, \operatorname{det}(F)=\prod_{i=1}^{n} \lambda_{i} ; \operatorname{allg} . \operatorname{Sp}\left(F^{k}\right)=\sum_{i=1}^{n} \lambda_{i}^{k} .
$$

Somit erhalten wir schließlich

$$
\operatorname{Sp}\left(F^{k}\right)=2^{k} .
$$

Zur Berechnung der $k$-ten Potenz von $\left(\tilde{C}^{-1} \tilde{U} \tilde{U}^{T}\right)$ befassen wir uns zunächst mit der Matrixstruktur. Diese besitzt aufgrund der $2 \tilde{n}$-Periodizität die folgende Blockstruktur (vergl. (B.14) im Anhang B.3):

$$
\tilde{C}^{-1} \tilde{U} \tilde{U}^{T}=\left(\begin{array}{ccc}
\tilde{A}_{1} & \ldots & \tilde{A}_{1} \\
\tilde{A}_{2} & \ldots & \tilde{A}_{2} \\
\vdots & \vdots & \vdots \\
\tilde{A}_{M} & \ldots & \tilde{A}_{M}
\end{array}\right) \in \mathcal{M}(2 M \tilde{n} \times 2 M \tilde{n}, \mathbb{R})
$$

mit den Matrixelementen der $2 \tilde{n} \times 2 \tilde{n}$-Blockmatrix $\tilde{A}_{\mu}$ :

$$
\tilde{a}_{m n}^{\mu}=\left(\frac{g \tilde{n}}{2}\right) \cdot\left(c_{(\mu-1)(2 \tilde{n})+m}^{-1}\left(\delta_{m-n, 0}-\delta_{m+n, 2 \tilde{n} \mathbb{N}^{*}}\right)\right), \mathbb{N}^{*}:=\{1,2\}
$$

und der Blockmatrix-Zahl $\mu=1,2, \ldots, M$ sowie den Zeilen- und Spaltenindizes innerhalb der Blockmatrix $m, n=1,2, \ldots, 2 \tilde{n}$. Das bedeutet, in der BlockmatrixSchreibweise liest sich $\tilde{C}^{-1} \tilde{U} \tilde{U}^{T}$ als eine $M \times M$-Matrix mit $2 \tilde{n} \times 2 \tilde{n}$-Blockmatrizen 
als deren Matrixelemente. Die Matrixmultiplikation der Blockmatrizen verläuft analog zur Multiplikation von gewöhnlichen Matrizen [42,107]. Zunächst führen wir eine neue Matrix ein, die wie folgt definiert ist:

$$
\tilde{\Sigma}:=\sum_{\mu=1}^{M} \tilde{A}_{\mu}, \tilde{\Sigma} \in \mathcal{M}(2 \tilde{n} \times 2 \tilde{n}, \mathbb{R}) .
$$

Die dazugehörigen Matrixelemente sind

$$
\tilde{\sigma}_{m n}=\left(\frac{g \tilde{n}}{2}\right) \cdot \sum_{\mu=1}^{M} c_{(\mu-1)(2 \tilde{n})+m}^{-1}\left(\delta_{m-n, 0}-\delta_{m+n, 2 \tilde{n} \mathbb{N}^{*}}\right)=: S_{m}\left(\delta_{m-n, 0}-\delta_{m+n, 2 \tilde{n} \mathbb{N}^{*}}\right) .
$$

Wir erhalten damit für die $k$-te Potenz von $\left(\tilde{C}^{-1} \tilde{U} \tilde{U}^{T}\right)$ :

$$
\left(\tilde{C}^{-1} \tilde{U} \tilde{U}^{T}\right)^{k}=\left(\begin{array}{ccc}
\tilde{A}_{1} \tilde{\Sigma}^{k-1} & \ldots & \tilde{A}_{1} \tilde{\Sigma}^{k-1} \\
\tilde{A}_{2} \tilde{\Sigma}^{k-1} & \ldots & \tilde{A}_{2} \tilde{\Sigma}^{k-1} \\
\vdots & \vdots & \vdots \\
\tilde{A}_{M} \tilde{\Sigma}^{k-1} & \ldots & \tilde{A}_{M} \tilde{\Sigma}^{k-1}
\end{array}\right)
$$

Nun liegt es nahe, zunächst die Potenzen von $\tilde{\Sigma}$ zu bestimmen. Wir erhalten für $\tilde{\Sigma}^{2}$ die Matrixelemente

$$
\begin{aligned}
\left(\tilde{\sigma}^{2}\right)_{m n} & =S_{m} \sum_{l=1}^{2 \tilde{n}}\left(\delta_{m-l, 0}-\delta_{m+l, 2 \tilde{n} \mathbb{N}^{*}}\right)\left(\delta_{l-n, 0}-\delta_{l+n, 2 \tilde{n} \mathbb{N}^{*}}\right) S_{l} \\
& =S_{m} \sum_{l=1}^{2 \tilde{n}}\left(\delta_{m, l} \delta_{l, n}+\delta_{l, 2 \tilde{n} \mathbb{N}^{*}-m} \delta_{l, 2 \tilde{n} \mathbb{N}^{*}-n}-\delta_{l, n} \delta_{l, 2 \tilde{n} \mathbb{N}^{*}-m}-\delta_{m, l} \delta_{l, 2 \tilde{n} \mathbb{N}^{*}-n}\right) S_{l} \\
& =S_{m}\left(S_{n}+S_{2 \tilde{n}-n}\right)\left(\delta_{n-m, 0}-\delta_{n+m, 2 \tilde{n} \mathbb{N}^{*}}\right) .
\end{aligned}
$$

Hierbei sieht man leicht, dass beim Quadrieren die Besetzungsstruktur der Matrix $\left(\tilde{C}^{-1} \tilde{U} \tilde{U}^{T}\right)$ aufgrund des Terms $\left(\delta_{n-m, 0}-\delta_{n+m, 2 \tilde{n} \mathbb{N}^{*}}\right)$ erhalten bleibt. Das bedeutet, dass alle Matrixelemente der Haupt- und Nebendiagonalen (für deren Indizes $(m-n) \in 2 \tilde{n} \mathbb{Z}$ $\wedge(m+n) \notin 2 \tilde{n} \mathbb{Z}$ gilt $)$ durch das Potenzieren weiterhin positiv, die Matrixelemente der Haupt- und Neben-Gegendiagonalen (für deren Indizes $(m+n) \in 2 \tilde{n} \mathbb{Z} \wedge(m-n) \notin 2 \tilde{n} \mathbb{Z}$ gilt) dagegen weiterhin negativ bleiben. Alle restlichen Einträge bleiben Null.

Nun können wir sukzessive alle weiteren Potenzen von $\tilde{\Sigma}$ analog zu 3.43 ausrechnen und erhalten für die entsprechenden Matrixelemente für $k \geq 4$ :

$$
\begin{aligned}
\left(\tilde{\sigma}^{k-1}\right)_{m n} & =S_{m}\left(S_{n}-S_{2 \tilde{n}-n}\right)^{k-3} \sum_{l=1}^{2 \tilde{n}}\left(\delta_{m-l, 0}-\delta_{m+l, 2 \tilde{n} \mathbb{N}^{*}}\right)\left(\delta_{l-n, 0}-\delta_{l+n, 2 \tilde{n} \mathbb{N}^{*}}\right) S_{l} \\
& =S_{m}\left(S_{n}+S_{2 \tilde{n}-n}\right)^{k-2}\left(\delta_{n, m}-\delta_{n+m, 2 \tilde{n} \mathbb{N}^{*}}\right)
\end{aligned}
$$


Auch das Matrixprodukt $\tilde{A}_{\mu} \cdot \tilde{\Sigma}^{k-1}$ lässt sich in gleicher Weise berechnen, wir geben die Matrixelemente dazu an:

$$
\begin{aligned}
\left(\tilde{a}^{\mu} \tilde{\sigma}^{k-1}\right)_{m n} & =c_{\mu(2 \tilde{n})+m}^{-1} \sum_{l=1}^{2 \tilde{n}}\left(\delta_{m, l}-\delta_{m+l, 2 \tilde{n} \mathbb{N}^{*}}\right)\left(S_{n}+S_{2 \tilde{n}-n}\right)^{k-2}\left(\delta_{n, l}-\delta_{n+l, 2 \tilde{n} \mathbb{N}^{*}}\right) S_{l} \\
& =c_{\mu(2 \tilde{n})+m}^{-1}\left(S_{n}+S_{2 \tilde{n}-n}\right)^{k-1}\left(\delta_{n, m}-\delta_{n+m, 2 \tilde{n} \mathbb{N}^{*}}\right) .
\end{aligned}
$$

Mit Hilfe eines weiteren Indexes $b=1,2, \ldots, N$ können wir die Symmetrie bezüglich $2 \tilde{n}$ ausnutzen und erhalten für die Spur

$$
\operatorname{Sp}\left(\tilde{C}^{-1} \tilde{U} \tilde{U}^{T}\right)^{k}=\left(\frac{g \tilde{n}}{2}\right) \cdot \sum_{\mu=1}^{M} \sum_{b=1}^{\tilde{n}-1}\left(c_{\tilde{n}(2 \mu-1)-b}^{-1}+c_{\tilde{n}(2 \mu-1)+b}^{-1}\right)\left(S_{\tilde{n}-b}+S_{\tilde{n}+b}\right)^{k-1},
$$

und mit der Definition von $S_{m}$ aus (3.41) folgern wir

$$
\begin{aligned}
& \operatorname{Sp}\left(\tilde{C}^{-1} \tilde{U} \tilde{U}^{T}\right)^{k} \\
& =\left(\frac{g \tilde{n}}{2}\right)^{k} \cdot \sum_{\mu=1}^{M} \sum_{b=1}^{\tilde{n}-1}\left(c_{\tilde{n}(2 \mu-1)-b}^{-1}+c_{\tilde{n}(2 \mu-1)+b}^{-1}\right)\left(\sum_{\mu^{\prime}=1}^{M}\left(c_{\tilde{n}\left(2 \mu^{\prime}-1\right)-b}^{-1}+c_{\tilde{n}\left(2 \mu^{\prime}-1\right)+b}^{-1}\right)\right)^{k-1} \\
& =\left(\frac{g \tilde{n}}{2}\right)^{k} \cdot \sum_{\mu=1}^{M}\left(\sum_{b=1}^{\tilde{n}-1}\left(c_{\tilde{n}(2 \mu-1)-b}^{-1}+c_{\tilde{n}(2 \mu-1)+b}^{-1}\right)\right)^{k},
\end{aligned}
$$

und erhalten damit zu guter Letzt mit (3.34) sowie (3.37) und der Vertauschung der Summen die gewünschte Spur (siehe Veröffentlichung von A. v. d. Heydt, D. Wilkin, P. Benetatos und A. Zippelius [51])

$$
\operatorname{Sp}\left(C^{-1} U U^{T}\right)^{k}=(g \tilde{n})^{k} \cdot \sum_{b=1}^{\tilde{n}-1}\left(\sum_{\mu=1}^{\infty}\left(c_{\tilde{n}(2 \mu-1)-b}^{-1}+c_{\tilde{n}(2 \mu-1)+b}^{-1}\right)\right)^{k} .
$$

Hierbei wurde die Summation über alle Modenzahlen ausgeführt $(M \rightarrow \infty)$.

\subsection{Ergebnis der Force-Extension mit Hilfe von $\mathcal{Z}_{\text {rel }}$}

Wir setzen das Ergebnis aus dem vorigen Kapitel 3.5. Gleichung (3.48), in die relative Zustandssumme (3.31) ein. Es zeigt sich, dass $\mathcal{Z}_{\text {rel }}$ in $N$ Faktoren faktorisiert (siehe auch [51]) . Mit

$$
f_{b}:=(g \tilde{n}) \cdot \sum_{\mu=1}^{\infty}\left(c_{\tilde{n}(2 \mu-1)-b}^{-1}+c_{\tilde{n}(2 \mu-1)+b}^{-1}\right) ; b=1,2, \ldots, N
$$

finden wir

$$
\begin{aligned}
\mathcal{Z}_{\mathrm{rel}} & =\exp \left\{-\frac{1}{2} \sum_{k=1}^{\infty}\left[\frac{(-1)^{k+1}}{k}\left(\sum_{b=1}^{\tilde{n}-1}\left(f_{b}\right)^{k}\right)\right]\right\}=\prod_{b=1}^{\tilde{n}-1} \exp \left(-\frac{1}{2}\left(\log \left(1+f_{b}\right)\right)\right) \\
& =\prod_{b=1}^{\tilde{n}-1}\left(1+f_{b}\right)^{-\frac{1}{2}}=: \prod_{b=1}^{\tilde{n}-1}(\mathscr{Z}(b, \tilde{n}, z))^{-\frac{1}{2}}
\end{aligned}
$$


mit dem Faktor

$$
\mathscr{Z}(b, \tilde{n}, z)=1-\left(\frac{\tilde{g} \tilde{T}}{z^{3 / 2}}\right) \cdot \frac{\sinh \left(\frac{\sqrt{z}}{\tilde{n}}\right)}{\cos \left(\frac{\pi b}{\tilde{n}}\right)+\cosh \left(\frac{\sqrt{z}}{\tilde{n}}\right)}+\frac{\tilde{g} \tilde{T}}{2 z \tilde{n}}\left(1+\tan ^{2}\left(\frac{\pi b}{2 \tilde{n}}\right)\right) .
$$

Dieses Zwischenergebnis ermöglicht uns nun die Ermittlung der Force-Extension-Relation. Die exakte Lösung des crosslinkabhängigen Anteils lautet mit $\hat{b}:=\frac{\pi b}{\tilde{n}}$

$$
\begin{aligned}
\left.\frac{\langle x(L)\rangle}{L}\right|_{\text {xlink }} & =\left(\frac{T}{2 L}\right) \cdot\left(\frac{\partial \log \mathcal{Z}_{\text {rel }}}{\partial f}\right)=-\left(\frac{T}{4 L}\right) \cdot \frac{\partial \log \left(\operatorname{det}\left(\mathbb{1}+C^{-1} U U^{T}\right)\right)}{\partial f} \\
& =-\left(\frac{T}{4 L}\right) \cdot \sum_{b=1}^{\tilde{n}-1} \frac{\partial_{f} \mathscr{Z}(b, \tilde{n}, z)}{\mathscr{Z}(b, \tilde{n}, z)}=\frac{\tilde{g} \tilde{T}^{2}}{4 z^{2}} \sum_{b=1}^{\tilde{n}-1} \frac{z(b, \tilde{n}, z)}{n(b, \tilde{n}, z)}
\end{aligned}
$$

mit den Termen $z(b, \tilde{n}, z)$ und $n(b, \tilde{n}, z)$, die wie folgt definiert sind:

$$
\begin{aligned}
z(b, \tilde{n}, z) & :=3 \tilde{n} \sinh \left(\frac{\sqrt{z}}{\tilde{n}}\right) \cos ^{2}\left(\frac{\hat{b}}{2}\right)\left(\cos (\hat{b})+\cosh \left(\frac{\sqrt{z}}{\tilde{n}}\right)\right) \\
& -\sqrt{z}\left[\left(\cos (\hat{b})+\cosh \left(\frac{\sqrt{z}}{\tilde{n}}\right)\right)^{2}+\cos ^{2}\left(\frac{\hat{b}}{2}\right)\left(1+\cosh \left(\frac{\sqrt{z}}{\tilde{n}}\right) \cos (\hat{b})\right)\right] \\
n(b, \tilde{n}, z) & :=\left(\cos (\hat{b})+\cosh \left(\frac{\sqrt{z}}{\tilde{n}}\right)\right)\left[\left(-2 \tilde{n} \sqrt{z} \cos ^{2}\left(\frac{\hat{b}}{2}\right)-\left(\frac{\tilde{g} \tilde{T}}{\sqrt{z}}\right)\right)\left(\cos (\hat{b})+\cosh \left(\frac{\sqrt{z}}{\tilde{n}}\right)\right)\right. \\
& \left.+\left(\frac{2 \tilde{n} \tilde{g} \tilde{T}}{z}\right) \sinh \left(\frac{\sqrt{z}}{\tilde{n}}\right) \cos ^{2}\left(\frac{\hat{b}}{2}\right)\right] .
\end{aligned}
$$

Die gesamte Force-Extension-Relation beinhaltet außer dem Crosslink-Term zusätzlich noch den Term für die Polymerketten, das heißt wir addieren dafür lediglich den MarkoSiggia-Term aus (3.24) und erhalten:

$$
\frac{\langle x(L)\rangle}{L}=1-\frac{\tilde{T}}{4 z}(\sqrt{z} \operatorname{coth}(\sqrt{z})-1)+\frac{\tilde{g} \tilde{T}^{2}}{4 z^{2}} \sum_{b=1}^{\tilde{n}-1} \frac{z(b, \tilde{n}, z)}{n(b, \tilde{n}, z)} .
$$

In den beiden Abbildungen (3.2) und (3.3) ist das Verhalten der Force-ExtensionRelation $\left(\langle x(L)\rangle \cdot L^{-1}\right)$ bzw. deren Crosslink-Anteil $\left(\left.\langle x(L)\rangle \cdot L^{-1}\right|_{\text {xlink }}\right)$ als Funktion der Kraft $z$ für eine unterschiedliche Anzahl $N$ von Crosslinks dargestellt. Für das Verhältnis von Kontur- zu Persistenzlänge gilt nach (3.1) und (3.22) die Relation $L / L_{P}=\tilde{T} / 2$.

Man kann aus den Kurven schließen, dass mit zunehmender Crosslinkzahl das System immer steifer wird, der Zuwachs jedoch nicht linear verläuft. Genauer gesagt trägt jeder weitere hinzugefügte Crosslink immer weniger zur Erhöhung der Steifigkeit bei. Außerdem werden die crosslinkzahlbedingten Unterschiede in der Force-Extension immer kleiner, je höher die Zugkraft ist. 


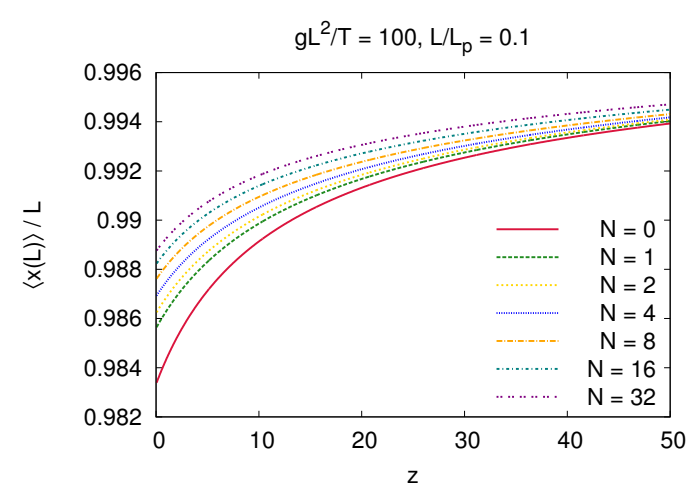

Abbildung 3.2.: Force-Extension $\langle x(L)\rangle \cdot L^{-1}$ als Funktion von $z$ für verschiedene Crosslinkzahlen $N$

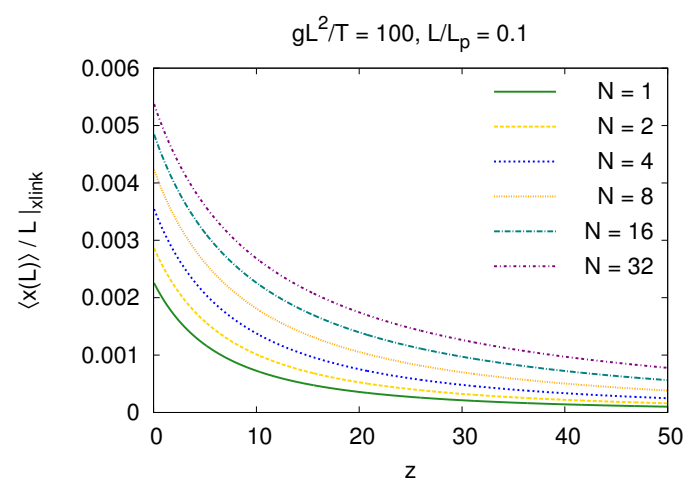

Abbildung 3.3.: Crosslinkabh. Anteil der Force-Extension $\left.\langle x(L)\rangle \cdot L^{-1}\right|_{\text {xlink }}$ als Funktion von $z$ für verschiedene Crosslinkzahlen $N$

Nun legen wir das Augenmerk auf den Fall variabler Crosslink-Stärken $g$ bei einer konstanten Crosslinkzahl $N$ (siehe Abbildung (3.4)). Auch hierbei lässt sich mit zunehmendem $\tilde{g}$ eine nichtlinear abnehmende Elastizität bzw. wachsende Steifigkeit des Modellsystems feststellen. Offensichtlich existiert eine Grenzkurve für den Grenzwert harter Crosslinks, woraus gefolgert werden kann, dass die Steifigkeit des Systems durch die Erhöhung von $g$ nicht beliebig gesteigert werden kann.

\section{Harte Crosslinks}

Im Limes harter Crosslinks (Hard-Crosslink-Limit, $\tilde{g} \rightarrow \infty$ ) vereinfacht sich der Ausdruck 3.52 ein wenig, das heißt, es gilt in diesem Falle

$$
\left.\frac{\langle x(L)\rangle}{L}\right|_{\mathrm{xlink}, \tilde{g} \rightarrow \infty}=\frac{\tilde{T}}{4 z^{2}} \sum_{b=1}^{\tilde{n}-1} \frac{z(b, \tilde{n}, z)}{n_{g}(b, \tilde{n}, z)},
$$

mit

$$
\begin{aligned}
n_{g}(b, \tilde{n}, z):= & -\left(\frac{1}{\sqrt{z}}\right)\left(\cos (\hat{b})+\cosh \left(\frac{\sqrt{z}}{\tilde{n}}\right)\right)^{2} \\
& +\left(\frac{2 \tilde{n}}{z}\right) \sinh \left(\frac{\sqrt{z}}{\tilde{n}}\right) \cos ^{2}\left(\frac{\hat{b}}{2}\right)\left(\cos (\hat{b})+\cosh \left(\frac{\sqrt{z}}{\tilde{n}}\right)\right)
\end{aligned}
$$

wobei man direkt sehen kann, dass in diesem Grenzfall die Force-Extension unabhängig von $\tilde{g}$ ist. Für den Fall $N=8$ ist in Abbildung (3.5) die Force-Extension für harte Crosslinks über der Kraft $z$ aufgetragen. Während sich bei kleinen Crosslink-Stärken die verschiedenen Force-Extension-Kurven für große Werte von $z$ rasch annähern (siehe Abbildung (3.2) ), sind die durch $N$ bedingten Unterschiede im Kurvenverlauf bei harten Crosslinks immer noch stark ausgeprägt. 


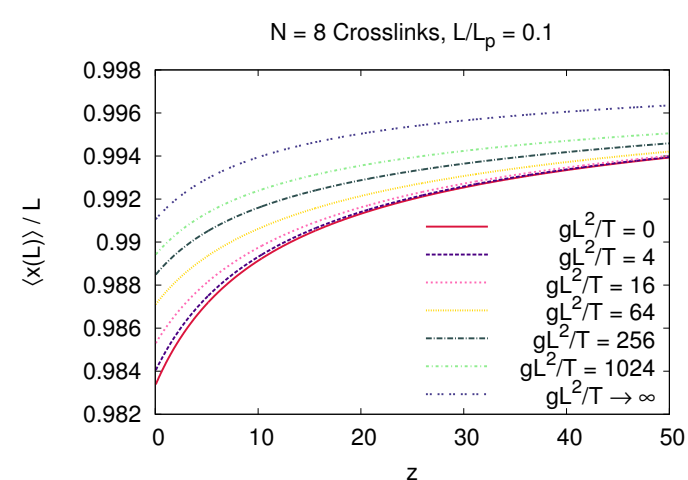

Abbildung 3.4.: Force-Extension $\langle x(L)\rangle \cdot L^{-1}$ als Funktion von $z$ für verschiedene Crosslinkstärken $\tilde{g}$

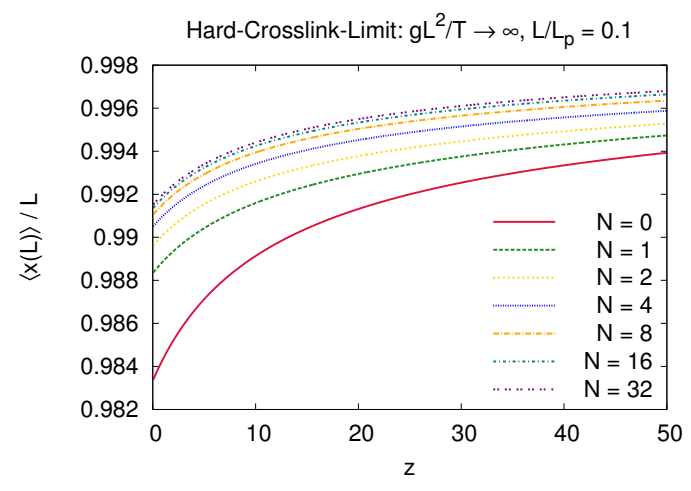

Abbildung 3.5.: Force-Extension $\left.\langle x(L)\rangle \cdot L^{-1}\right|_{\tilde{g} \rightarrow \infty}$ als Funktion von $z$ im Hard-Crossink-Limit für verschiedene $N$

\section{Strong-Stretching-Limit}

Eine andere, weitere Vereinfachung für den crosslinkabhängigen Anteil der Force-Extension erhalten wir, indem wir den Grenzfall hoher Zugkräfte betrachten, das heißt dabei gilt $z \gg 1$. Dieser Fall wird Strong-Stretching-Limit (ssl) genannt, er führt uns zu dem folgenden Resultat:

$$
\left.\frac{\langle x(L)\rangle}{L}\right|_{\mathrm{xlink}, \mathrm{ssl}}=\frac{1}{2} \sum_{b=1}^{\tilde{n}-1} \frac{\tilde{T}\left(1-\left(\frac{3 \tilde{n}}{\sqrt{z}}\right) \cos ^{2}\left(\frac{\pi b}{2 \tilde{n}}\right)\right)}{\sqrt{z}\left\{\left[-2 \tilde{n} \cos ^{2}\left(\frac{\pi b}{2 \tilde{n}}\right)+z\right]+2 \tilde{n}\left(\frac{z^{3 / 2}}{\tilde{g} \tilde{T}}\right) \cos ^{2}\left(\frac{\pi b}{2 \tilde{n}}\right)\right\}} .
$$

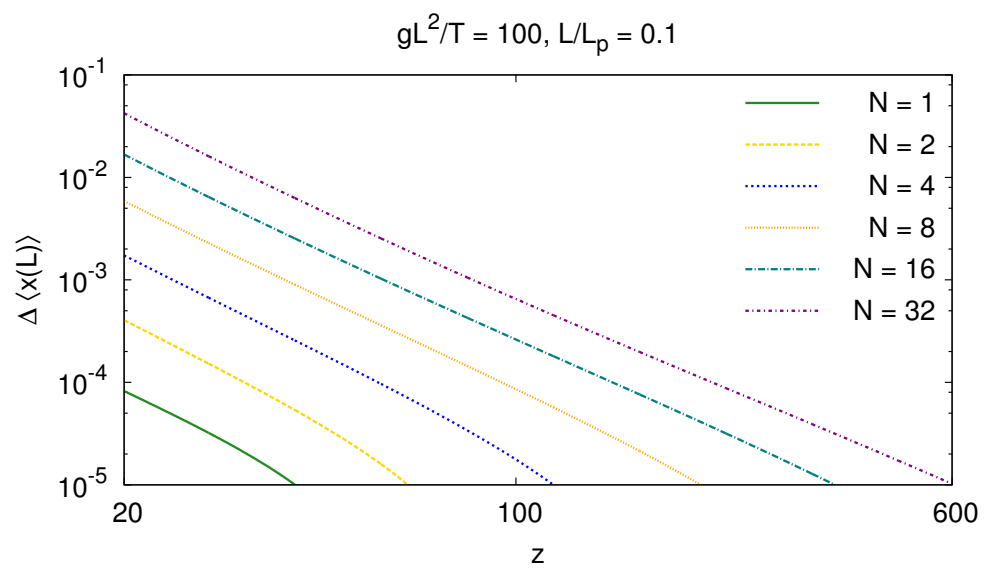

Abbildung 3.6.: Relativer Fehler $\triangle\langle x(L)\rangle$ zwischen exakter Lösung und Strong-StretchingLimit in Abhängigkeit von $z$

Um eine Vorstellung von der Größenordnung des Fehlers zu erhalten, die mit der Näherungslösung des Strong-Stretching-Limits gegenüber der exakten Lösung gemacht wird, ermitteln wir den relativen Fehler $\triangle\langle x(L)\rangle$ zwischen der exakten Lösung $\left(\langle x(L)\rangle \cdot L^{-1}\right)$ 
und der des Strong-Stretching-Limits:

$$
\langle x(L)\rangle_{\mathrm{ssl}} \cdot L^{-1}=\left.\langle x(L)\rangle \cdot L^{-1}\right|_{\mathrm{MS}, \mathrm{ssl}}+\left.\langle x(L)\rangle \cdot L^{-1}\right|_{\mathrm{xlink}, \mathrm{ssl}},
$$

das heißt, wir berechnen mit $\left.\langle x(L)\rangle \cdot L^{-1}\right|_{\mathrm{MS}, \mathrm{ssl}}=1-\frac{\tilde{T}}{4 z}(\sqrt{z}-1)$

$$
\triangle\langle x(L)\rangle:=\frac{|\langle x(L)\rangle|_{\mathrm{ssl}}-\langle x(L)\rangle \mid}{\langle x(L)\rangle} .
$$

Das Ergebnis aus (3.59) ist in Abbildung (3.6) für eine unterschiedliche Anzahl $N$ an Crosslinks in Abhängigkeit von $z$ aufgetragen. Offenbar wird der Fehler sowohl bei Verringerung der Crosslinkzahl $N$ als auch bei Erhöhung von $z$ schnell kleiner. In Anbetracht des sehr kleinen numerischen Fehlerwertes kann von einer sehr guten Näherung gesprochen werden.

\subsection{Berechnung von $G^{-1}$ mit Hilfe des Theorems von Cayley-Hamilton}

Das Modellsystem mit einer beliebigen Anzahl $N$ an Crosslinks lässt sich mit Hilfe der Zustandssumme $\mathcal{Z}$ lösen (siehe Kapitel 3.4 bis 3.6). In diesem Kapitel wird eine alternative Methode vorgestellt, die zur Bestimmung der Force-Extension-Relation des eingeführten Modellsystems mit $N$ Crosslinks verwendet werden kann. Das Ziel dieser Methode besteht darin, die Matrix $G$ (siehe (3.12) ) mit Hilfe des Theorems von CayleyHamilton zu invertieren, um über die Relationen (3.17) und (3.18) das angestrebte Ergebnis zu erhalten.

Das Theorem von Cayley-Hamilton besagt, dass jede quadratische $n \times n$-Matrix $B$ ihrem charakteristischen Polynom $f_{B}(\lambda):=\operatorname{det}\left(\lambda \mathbb{1}_{n}-B\right)$ genügt 29,36 . Die $n \times n$-Einheitsmatrix wird dabei mit $\mathbb{1}_{n}$ und die $n \times n$-Nullmatrix mit $0_{n}$ bezeichnet. Ist also

$$
f_{B}(\lambda)=(-1)^{n}\left[\lambda^{n}-p_{1} \lambda^{n-1}+p_{2} \lambda^{n-2}+\ldots+(-1)^{n} p_{n}\right]
$$

die charakteristische Funktion einer solchen Matrix $B$, so gilt

$$
f(B)=(-1)^{n}\left[B^{n}-p_{1} B^{n-1}+p_{2} B^{n-2}+\ldots+(-1)^{n} p_{n} \mathbb{1}_{n}\right]=0_{n}
$$

und damit für die Inverse 29

$$
B^{-1}=\frac{(-1)^{n-1}}{\operatorname{det}(B)}\left[B^{n-1}-p_{1} B^{n-2}+p_{2} B^{n-3}-\ldots+(-1)^{n-1} p_{n-1} \mathbb{1}_{n}\right] .
$$

Zur Berechnung der Matrix $B^{-1}$ sind dementsprechend zuerst die Koeffizienten des charakteristischen Polynoms $p_{1}, p_{2}, \ldots, p_{n}$ zu berechnen. Sind $\lambda_{1}, \lambda_{2}, \ldots, \lambda_{n}$ die charakteristischen Wurzeln der Matrix $B$, das heißt, gilt

$$
f_{B}(\lambda)=\left(\lambda-\lambda_{1}\right)\left(\lambda-\lambda_{2}\right) \cdots\left(\lambda-\lambda_{n}\right),
$$


so kann gezeigt werden, dass die Potenzen $\lambda_{1}^{k}, \lambda_{2}^{k}, \ldots, \lambda_{n}^{k}$ die charakteristischen Wurzeln der Matrix $B^{k}$ sind $(k=1,2, \ldots)$ 36. Folglich kann daraus die Spur der Matrix $B^{k}$ abgeleitet werden:

$$
\operatorname{Sp}\left(B^{k}\right)=s_{k}=\sum_{i=1}^{n} \lambda_{i}^{k}, k=1,2, \ldots
$$

Zwischen den Spuren der Matrizen $B, B^{2}, \ldots, B^{n}$ und den Koeffizienten des charakteristischen Polynoms existiert eine Relation. So werden nach der Methode von U. LE VERRIER die Spuren $s_{k}$ zur sukzessiven Berechnung der Koeffizienten $p_{1}, p_{2}, \ldots, p_{n}$ mit Hilfe der Newtonschen Formeln herangezogen. Genauer gilt 29,33

$$
\begin{aligned}
p_{1} & =s_{1} \\
p_{2} & =\left(p_{1} s_{1}-s_{2}\right) / 2 \\
p_{3} & =\left(p_{2} s_{1}-p_{1} s_{2}+s_{3}\right) / 3 \\
& \vdots \\
p_{n} & =\left(p_{n-1} s_{1}-p_{n-2} s_{2}+\ldots+(-1)^{n-1} s_{n}\right) / n .
\end{aligned}
$$

\subsubsection{Rechenschritte}

Wir betrachten zunächst die zu invertierende quadratische Matrix

$$
G=C+U U^{T}=C\left(\mathbb{1}_{4 M \tilde{n}}+C^{-1} U U^{T}\right) \Rightarrow G^{-1}=\left(\mathbb{1}_{4 M \tilde{n}}+C^{-1} U U^{T}\right)^{-1} C^{-1},
$$

mit der Matrix $B:=\left(\mathbb{1}_{4 M \tilde{n}}+C^{-1} U U^{T}\right) \in \mathcal{M}(4 M \tilde{n} \times 4 M \tilde{n}, \mathbb{R})$. Um die Inverse der Matrix $G$ zu bestimmen, bietet es sich an, zunächst $B^{-1}$ nach der oben erwähnten Vorgehensweise abzuleiten.

Mit Hilfe des Formalismus der Blockmatrizen [42,107] sind wir in der Lage, die Matrix $B$ in einer übersichtlicheren Form darzustellen. Mit der $4 \tilde{n} \times 4 \tilde{n}$-Einheitsmatrix $\mathbb{1}_{4 \tilde{n}}:=E$ sowie den $4 \tilde{n} \times 4 \tilde{n}$-Blockmatrizen $A_{1}, A_{2}, \ldots$ können wir sie schreiben als

$$
B=\left(\begin{array}{cccc}
E+A_{1} & A_{1} & \ldots & A_{1} \\
A_{2} & E+A_{2} & \ldots & A_{2} \\
\vdots & \vdots & \ddots & \vdots \\
A_{M} & A_{M} & \ldots & E+A_{M}
\end{array}\right)
$$

Die Blockmatrizen $A_{\mu}$ können mit Hilfe des Kronecker-Produktes wie folgt ausgedrückt werden: $A_{\mu}=\tilde{A}_{\mu} \otimes\left(\begin{array}{cc}1 & -1 \\ -1 & 1\end{array}\right)$, mit den Elementen der $2 \tilde{n} \times 2 \tilde{n}$-Blockmatrix $\tilde{A}_{\mu}$ aus 3.39 in Kapitel 3.5

$$
\tilde{a}_{m n}^{\mu}=\left(\frac{g \tilde{n}}{2}\right) \cdot\left(c_{(\mu-1)(2 \tilde{n})+m}^{-1}\left(\delta_{m-n, 0}-\delta_{m+n, 2 \tilde{n} \mathbb{N}^{*}}\right)\right), \mathbb{N}^{*}=\{1,2\},
$$

und der Blockmatrix-Zahl $\mu=1,2, \ldots, M$ sowie den Zeilen- und Spaltenindizes innerhalb einer Blockmatrix $m, n=1,2, \ldots, 2 \tilde{n}$. Zur Kennzeichnung der „BlockmatrixElemente" verwenden wir neben $\mu$ einen weiteren Index $\nu=1,2, \ldots, M$. So schreiben 
wir für die in (3.67) auftretenden Blockmatrizen

$$
B_{\mu \nu}=\delta_{\mu \nu} E+A_{\mu}=\delta_{\mu \nu} E+A_{\mu} \cdot T_{0} \quad \text { mit } \quad T_{0}:=E .
$$

Wir berechnen zunächst beliebige Potenzen der Matrix $B$ und erhalten für die „Blockmatrix-Elemente" der zweiten Potenz

$$
\begin{aligned}
\left(B^{2}\right)_{\mu \nu} & =\sum_{j=1}^{M}\left(\delta_{\mu j} E+A_{\mu} T_{0}\right)\left(\delta_{j \nu} E+A_{j}\right)=\delta_{\mu \nu} E+A_{\mu}(\Sigma+2 E) \\
& =: \delta_{\mu \nu} E+A_{\mu} T_{1}
\end{aligned}
$$

mit $\Sigma:=\sum_{\nu=1}^{M} A_{\nu}$. Höhere Potenzen können nun sukzessive mittels wiederholter Matrixmultiplikation hergeleitet werden, wobei sich die „Blockmatrix-Elemente“ der $k$-ten Potenz $\left(B^{k}\right)_{\mu \nu}$ durch den Term $T_{k-1}$ charakterisieren lassen. So erhalten wir für die $k$-te Potenz von $B$ allgemein für $k \geq 2$

$$
\begin{aligned}
\left(B^{k}\right)_{\mu \nu} & =\sum_{j=1}^{M}\left(\delta_{\mu j} E+A_{\mu} T_{k-2}\right)\left(\delta_{j \nu} E+A_{j}\right) \\
& =\delta_{\mu \nu} E+A_{\mu}\left(T_{k-2} \Sigma+E+T_{k-2}\right)=: \delta_{\mu \nu} E+A_{\mu} T_{k-1} \\
& =\delta_{\mu \nu} E+A_{\mu} \Sigma^{-1}\left[(\Sigma+E)^{k}-E\right] .
\end{aligned}
$$

Für den Term $T_{k}$ gilt demnach im Allgemeinen die Relation

$$
T_{k}=\Sigma^{-1}\left[(\Sigma+E)^{k+1}-E\right] \text {, für } k=0,1,2, \ldots,
$$

was per vollständiger Induktion gezeigt werden kann. Als Induktionsanfang berechnen wir unmittelbar $T_{0}=E$ und $T_{1}=\Sigma+2 E$, in Übereinstimmung mit (3.69) und 3.700. Im darauffolgenden Schritt gilt es zu zeigen, dass $T_{k+1}=\Sigma^{-1}\left[(\Sigma+E)^{k+2}-E\right]$ für alle $k \geq 1$ gültig ist. Zum Beweis der Induktionsbehauptung verwenden wir die Beziehung $T_{k}=\left(T_{k-1} \Sigma+E+T_{k-1}\right) ; k=1,2, \ldots$, aus (3.71) im ersten und die Induktionsvoraussetzung 3.72 im zweiten Rechenschritt:

$$
\begin{aligned}
T_{k+1}=T_{k} \Sigma+E+T_{k} & =\Sigma^{-1}\left[(\Sigma+E)^{k+1}-E\right] \Sigma+E+\Sigma^{-1}\left[(\Sigma+E)^{k+1}-E\right] \\
& =\Sigma^{-1}\left[(\Sigma+E)^{k+2}-E\right] .
\end{aligned}
$$

Schließlich finden wir für die Spur der Matrix $B^{k}$ mit $\bar{E}:=\sum_{\nu=1}^{M} E$ in BlockmatrixNotation:

$$
\begin{aligned}
\operatorname{Sp}\left(B^{k}\right) & =s_{k}=\sum_{\nu=1}^{M}\left(E+A_{\nu} \cdot T_{k-1}\right)=\bar{E}+\left[(\Sigma+E)^{k}-E\right] \\
& =4 M \tilde{n}+\sum_{b=1}^{\tilde{n}-1}\left[\left(1+2 \cdot\left(\frac{g \tilde{n}}{2}\right) \cdot \sum_{\mu=0}^{\infty}\left(c_{2 \tilde{n} \mu+\tilde{n}-b}^{-1}+c_{2 \tilde{n} \mu+\tilde{n}+b}^{-1}\right)\right)^{k}-1\right] .
\end{aligned}
$$


Im Einklang mit dem Formalismus für die Blockmatrizen berechnen wir auch die Koeffizienten bezogen auf die Blockmatrizen und bezeichnen diese aus diesem Grunde mit $\tilde{p}_{k}$. Zur Bestimmung der Koeffizienten $\tilde{p}_{1}, \ldots, \tilde{p}_{n}$ gehen wir von der $4 M \tilde{n} \times 4 M \tilde{n}$ Matrix $B$ aus, wir definieren in diesem Zusammenhang $\bar{E}$ als die endliche Summe von $M 4 \tilde{n} \times 4 \tilde{n}$-Einheitsmatrizen $\mathbb{1}_{4 \tilde{n}}: \bar{E}=M \cdot E$. Als Startwert der schrittweisen Berechnung von LE VERRIER kann unmittelbar $\tilde{p}_{1}=(\bar{E}+\Sigma)$ angegeben werden. Man kann daran erkennen, dass die Koeffizienten $\tilde{p}_{k}$ für $k \geq 2$ faktorisieren, das heißt, es gilt

$$
\tilde{p}_{k}=\left(\frac{1}{k !}\right)\left[\prod_{i=1}^{k-1}(\bar{E}-i \cdot E)\right] \cdot(\bar{E}+k \cdot \Sigma) \text { für } k=2,3, \ldots, M .
$$

Dies kann zusammenfassend und vereinfachend für alle $k=1,2, \ldots, M$ in der folgenden Weise geschrieben werden:

$$
\tilde{p}_{k}=\left(\begin{array}{c}
M \\
k
\end{array}\right)\left[E+\left(\frac{k}{M}\right) \Sigma\right]
$$

Insbesondere für den Spezialfall $k=M$ finden wir: $\tilde{p}_{M}=(E+\Sigma)$. Die Kenntnis der Koeffizienten $\tilde{p}_{M}$ aus 3.75 sowie die der „Blockmatrix-Elemente“ $\left(B^{k}\right)_{\mu \nu}$ aus 3.71) nutzen wir zur Bestimmung der Inversen von $B$ mit Hilfe des Theorems von CayleyHamilton (siehe Gleichung (3.62)). Wir erhalten nach einigen Rechenschritten für die Blockmatrix-Elemente von $B^{-1}$ den Term

$$
\left(B^{-1}\right)_{\mu \nu}=\frac{(1+\Sigma) \cdot \delta_{\mu \nu}-A_{\mu}}{1+\Sigma} .
$$

Kehren wir zur standardmäßigen Matrix-Indizierung zurück, so können wir nun die Diagonalelemente der Matrix $B^{-1}$ angeben. Dazu bietet es sich aus Gründen der Übersichtlichkeit an, die Abkürzung

$$
\mathscr{C}_{s}(\tilde{n}, b):=\sum_{\mu=0}^{\infty}\left(c_{\tilde{n}-b+2 \tilde{n} \mu}^{-s}+c_{\tilde{n}+b+2 \tilde{n} \mu}^{-s}\right), s \in\{1,2\}
$$

einzuführen. Die Diagonalelemente lauten dann mit $(3.78), b=1, \ldots,(\tilde{n}-1)$; $\mu=0,1,2, \ldots$, und $r \in\{0,1\}$

$$
b_{m m}^{-1}= \begin{cases}\frac{1+\left(\frac{g \tilde{n}}{2}\right) \cdot\left(2 \cdot \mathscr{C}_{1}(\tilde{n}, b)-c_{m}^{-1}\right)}{1+2 \cdot\left(\frac{g \tilde{n}}{2}\right) \cdot \mathscr{C}_{1}(\tilde{n}, b)} & , \text { für } m=2(\tilde{n} \pm b+2 \tilde{n} \mu)-r \\ 1 & , \text { für } m \quad \bmod 2 \tilde{n} \in\{2 \tilde{n}-1,0\} .\end{cases}
$$

Die Diagonalmatrix-Elemente sind demnach paarweise identisch, das heißt

$$
b_{2 m, 2 m}^{-1}=b_{2 m-1,2 m-1}^{-1} \forall m=1,2, \ldots .
$$

Die zur Bestimmung der Force-Extension-Relation benötigte Spur

$$
\operatorname{Sp}\left(G^{-1}\right)=\operatorname{Sp}\left(B^{-1} C^{-1}\right)
$$


kann nun mit Hilfe des Hadamard- oder Schur-Produktes ermittelt werden [16,54,55. Dabei wird die Spur des Matrixproduktes alternativ mit Hilfe des Vektorisierungsoperators ausgedrückt. Dieser Operator transformiert eine $m \times n$-Matrix in einen Spaltenvektor der Länge $m \cdot n$, indem die Spalten der Matrix der Reihe nach untereinander angeordnet werden. Sind zum Beispiel $\vec{a}_{1}, \vec{a}_{2}, \ldots, \vec{a}_{n}$ die jeweils $m$ Matrixelemente enthaltenden $n$ Spalten der Matrix $A \in \mathcal{M}(m \times n, \mathbb{R})$, so ergibt die Vektorisierung von A

$$
\operatorname{vec}(A)=\left(\vec{a}_{1}, \vec{a}_{2}, \ldots, \vec{a}_{n}\right)^{T} \in \mathbb{R}^{m n} .
$$

Die Spur des Produktes zweier Matrizen kann demgemäß als inneres Produkt zweier Vektoren angesehen werden. So erhalten wir schließlich

$$
\begin{aligned}
& \operatorname{Sp}\left(G^{-1}\right)=\operatorname{Sp}\left(B^{-1} C^{-1}\right) \\
& =\operatorname{Sp}\left(\left(C^{-1}\right)^{T} B^{-1}\right)=\operatorname{vec}\left(C^{-1}\right)^{T} \operatorname{vec}\left(B^{-1}\right)=\sum_{m, n}\left(C^{-1} \circ B^{-1}\right)_{m n} \\
& =\sum_{m, n} c_{m n}^{-1} b_{m n}^{-1}=\sum_{m} c_{m m}^{-1} b_{m m}^{-1} \\
& =\sum_{b=1}^{n-1}\left\{[ 1 + ( g \tilde { n } ) \mathscr { C } _ { 1 } ( \tilde { n } , b ) ] ^ { - 1 } \left\{\sum_{m=0}\left[1+\left(\frac{g \tilde{n}}{2}\right)\left(2 \mathscr{C}_{1}(\tilde{n}, b)-c_{\tilde{n}-b+2 \tilde{n} m}^{-1}\right)\right] 2 c_{\tilde{n}-b+2 \tilde{n} m}^{-1}\right.\right. \\
& \left.\left.+\sum_{m=0}\left[1+\left(\frac{g \tilde{n}}{2}\right)\left(2 \mathscr{C}_{1}(\tilde{n}, b)-c_{\tilde{n}+b+2 \tilde{n} m}^{-1}\right)\right] 2 c_{\tilde{n}+b+2 \tilde{n} m}^{-1}\right\}\right\}+2 \sum_{\mu=0} c_{\tilde{n}+\tilde{n} \mu}^{-1} .
\end{aligned}
$$

Dieses Ergebnis lässt sich weiter vereinfachen. Nach einer einfachen Umschreibung ergibt sich für (3.83)

$$
\begin{aligned}
\operatorname{Sp}\left(G^{-1}\right) & =2 \sum_{\mu=0} c_{\tilde{n}+\tilde{n} \mu}^{-1} \\
& +\sum_{b=1}^{\tilde{n}-1}\left\{\left[1+(g \tilde{n}) \cdot \mathscr{C}_{1}(\tilde{n}, b)\right]^{-1} \cdot\left\{\left[1+(g \tilde{n}) \mathscr{C}_{1}(\tilde{n}, b)\right] \cdot 2 \mathscr{C}_{1}(\tilde{n}, b)-(g \tilde{n}) \mathscr{C}_{2}(\tilde{n}, b)\right\}\right\} .
\end{aligned}
$$

Für die Force-Extension-Relation benötigen wir die Relation (siehe Gleichungen (3.49) bis (3.51)

$$
1+(g \tilde{n}) \cdot \mathscr{C}_{1}(\tilde{n}, b)=\mathscr{Z}(b, \tilde{n}, z)
$$

und darüber hinaus die folgenden weiteren Zwischenergebnisse:

$$
\begin{gathered}
\left(\frac{\tilde{T}}{2}\right) \cdot \sum_{\mu=0}^{\infty}\left(\pi^{2}(\tilde{n}+\tilde{n} \mu)^{2}+z\right)^{-1}=-\frac{\tilde{T}}{4 z}+\frac{\tilde{T} \operatorname{coth}\left(\frac{\sqrt{z}}{\tilde{n}}\right)}{4 \tilde{n} \sqrt{z}}, \\
\left(\frac{\tilde{T}}{2}\right) \cdot \sum_{\mu=1}^{\infty} \sum_{j=0}^{1}\left(\pi^{2}\left(\tilde{n}(2 \mu-1)+(-1)^{j} \cdot b\right)^{2}+z\right)^{-1}=\frac{\tilde{T}}{4 \tilde{n} \sqrt{z}} \cdot \frac{\sinh \left(\frac{\sqrt{z}}{\tilde{n}}\right)}{\cos \left(\frac{\pi b}{\tilde{n}}\right)+\cosh \left(\frac{\sqrt{z}}{\tilde{n}}\right)},
\end{gathered}
$$




$$
\begin{aligned}
& \left(\frac{\tilde{g} \tilde{n} \tilde{T}^{2}}{2}\right) \cdot \sum_{\mu=1}^{\infty} \sum_{j=0}^{1}\left(\pi^{2}\left(\tilde{n}(2 \mu-1)+(-1)^{j} \cdot b\right)^{2} \cdot\left[\pi^{2}\left(\tilde{n}(2 \mu-1)+(-1)^{j} \cdot b\right)^{2}+z\right]^{2}\right)^{-1} \\
& =\frac{\tilde{g} \tilde{T}^{2}}{8 \tilde{n} z^{2}} \cdot\left[\left(1+\tan ^{2}\left(\frac{\pi b}{2 \tilde{n}}\right)\right)+\frac{\left(1+\cos \left(\frac{\pi b}{\tilde{n}}\right) \cosh \left(\frac{\sqrt{z}}{\tilde{n}}\right)\right)}{\left(\cos \left(\frac{\pi b}{\tilde{n}}\right)+\cosh \left(\frac{\sqrt{z}}{\tilde{n}}\right)\right)^{2}}\right]-\frac{3 \tilde{g} \tilde{T}^{2}}{8 z^{5 / 2}} \cdot\left(\frac{\sinh \left(\frac{\sqrt{z}}{\tilde{n}}\right)}{\cos \left(\frac{\pi b}{\tilde{n}}\right)+\cosh \left(\frac{\sqrt{z}}{\tilde{n}}\right)}\right) .
\end{aligned}
$$

Zur übersichtlicheren Darstellung des Endergebnisses führen wir des Weiteren die Abkürzungen

$$
\begin{aligned}
& t_{1}(b, \tilde{n}, z):=\frac{\sinh \left(\frac{\sqrt{z}}{\tilde{n}}\right)}{\cos \left(\frac{\pi b}{\tilde{n}}\right)+\cosh \left(\frac{\sqrt{z}}{\tilde{n}}\right)} \quad ; \quad t_{2}(b, \tilde{n}):=\left(1+\tan ^{2}\left(\frac{\pi b}{2 \tilde{n}}\right)\right) \\
& t_{3}(b, \tilde{n}, z):=\frac{\left(1+\cos \left(\frac{\pi b}{\tilde{n}}\right) \cosh \left(\frac{\sqrt{z}}{\tilde{n}}\right)\right)}{\left(\cos \left(\frac{\pi b}{\tilde{n}}\right)+\cosh \left(\frac{\sqrt{z}}{\tilde{n}}\right)\right)^{2}}
\end{aligned}
$$

ein und erhalten schließlich für die Force-Extension-Relation des Systems

$$
\begin{aligned}
\frac{\langle x(L)\rangle}{L} & =1-\frac{1}{4} \sum_{m=1}^{\infty} q_{m}^{2} \cdot g_{m m}^{-1}=1+\frac{\tilde{T}}{4 z}-\frac{\tilde{T} \operatorname{coth}\left(\frac{\sqrt{z}}{\tilde{n}}\right)}{4 \tilde{n} \sqrt{z}} \\
& -\sum_{b=1}^{\tilde{n}-1}\left\{[ \mathscr { Z } ( b , \tilde { n } , z ) ] ^ { - 1 } \cdot \left\{[\mathscr{Z}(b, \tilde{n}, z)] \cdot\left(\frac{\tilde{T}}{4 \tilde{n} \sqrt{z}}\right) \cdot t_{1}(b, \tilde{n}, z)\right.\right. \\
& \left.\left.-\frac{\tilde{g} \tilde{T}^{2}}{8 \tilde{n} z^{2}} \cdot\left[t_{2}(b, \tilde{n})+t_{3}(b, \tilde{n}, z)\right]+\frac{3 \tilde{g} \tilde{T}^{2}}{8 z^{5 / 2}} \cdot t_{1}(b, \tilde{n}, z)\right\}\right\} .
\end{aligned}
$$

Der Ausdruck (3.91) enthält definitionsgemäß sowohl den Beitrag der WLCs als auch den der Crosslinks. Für den Spezialfall $\tilde{g}=0$ vereinfacht sich dieser erwartungsgemäß zum Marko-Siggia-Term, der in (3.24) gekennzeichnet ist. Somit haben wir eine weitere Methode zur Ermittlung der Force-Extension gefunden.

Um die beiden komplexen analytischen Ausdrücke (3.54) und (3.91), die durch die beiden bislang vorgestellten Lösungswege hervorgegangen sind, miteinander vergleichen zu können, werden die Resultate graphisch aufgetragen. Zur Überprüfung der beiden Ergebnisse sind in Abbildung (3.7) die aus der Zustandssumme (Z) (siehe Kapitel 3.6) und die mit Hilfe des Theorems von Cayley-Hamilton (C) (siehe hiesiges Kapitel 3.7) erhaltenen Force-Extension-Kurven für verschiedene Werte der Crosslinkzahl $N$ bei einem Verhältnis zwischen Kontur- und Persistenzlänge von $L / L_{P}=1.0$ aufgetragen. Offenbar führen beide Resultate zu identischen Force-Extension-Kurvenverläufen, das heißt, sowohl die Auswertung der relativen Zustandssumme wie auch die Anwendung des Theorems von Cayley-Hamilton liefern das gleiche Resultat.

Im Vergleich zu Abbildung (3.2) wurde in Abbildung (3.7) neben zum Teil abweichenden Crosslinkzahlen eine zehnfach niedrigere Persistenzlänge $L_{P}$ gewählt $\left(L / L_{P}=1.0\right)$. Die damit verbundene erhöhte Flexibilität des Systems führt zum Anwachsen der transversalen Fluktuationen und spiegelt sich unmittelbar in kleineren Extensionen, das heißt in kleineren mittleren End-zu-End-Abständen wider. 


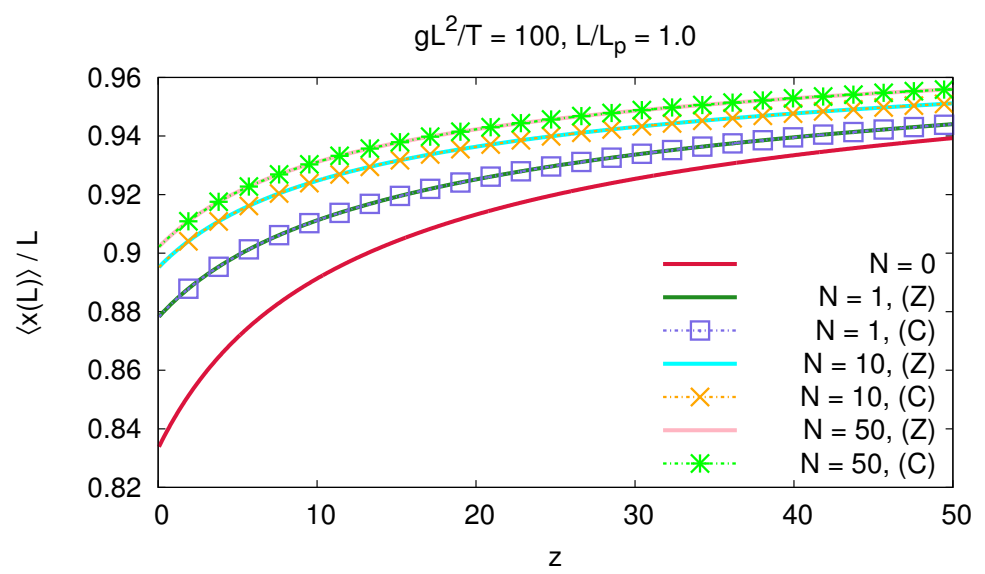

Abbildung 3.7.: Vergleich der Force-Extension-Ergebnisse $\langle x(L)\rangle \cdot L^{-1}$, gewonnen aus der Zustandssumme (Z) und mit Hilfe des Theorems von Cayley-Hamilton (C)

\subsection{Berechnung der Force-Extension mit Hilfe einer Reihenentwicklung}

Eine dritte und recht kurze Variante zur Kalkulation der Force-Extension-Relation für eine beliebige Zahl von $N=\tilde{n}-1$ Crosslinks lässt sich durch eine Reihenentwicklung für eine inverse Matrix herleiten [43. Das Ziel besteht auch hier darin, die Spur von $G^{-1}$ zu bestimmen. Unser Ausgangspunkt der Berechnung ist dementsprechend (siehe (3.66)):

$$
G^{-1}=\left(\mathbb{1}_{4 M \tilde{n}}+A\right)^{-1} C^{-1} \text {, mit } A:=C^{-1} U U^{T} .
$$

Wir entwickeln nun $\left(\mathbb{1}_{4 M \tilde{n}}+A\right)^{-1}$ in eine Reihe, so dass wir für 3.92 schreiben können:

$$
G^{-1}=\left(\mathbb{1}_{4 M \tilde{n}}+\sum_{k=1}^{\infty}(-A)^{k}\right) C^{-1} .
$$

Damit ist es ganz offenkundig, als nächstes die Spur der Summanden in (3.93) zu bestimmen. Wir finden zunächst mit den in (B.15) und (B.16) angegebenen Eigenschaften des Kronecker-Produktes (siehe auch (3.33) bis (3.35) und (3.37) in Kapitel 3.5)

$$
\operatorname{Sp}\left(A^{k} C^{-1}\right)=\operatorname{Sp}\left[\tilde{A}^{k} \tilde{C}^{-1} \otimes F^{k}\right]=\operatorname{Sp}\left(\tilde{A}^{k} \tilde{C}^{-1}\right) \cdot \operatorname{Sp}\left(F^{k}\right)=2^{k} \cdot \operatorname{Sp}\left(\tilde{A}^{k} \tilde{C}^{-1}\right) .
$$

Hiermit und mit Hilfe von (3.46) und der Abkürzung (3.78) ergibt sich weiter

$$
\begin{aligned}
\operatorname{Sp}\left(A^{k} C^{-1}\right) & =2^{k} \cdot\left(\frac{g \tilde{n}}{2}\right) \cdot \sum_{\mu=1}^{M} \sum_{b=1}^{\tilde{n}-1}\left(c_{\tilde{n}(2 \mu-1)-b}^{-2}+c_{\tilde{n}(2 \mu-1)+b}^{-2}\right)\left(S_{\tilde{n}-b}+S_{\tilde{n}+b}\right)^{k-1} \\
& =(g \tilde{n}) \cdot \sum_{b=1}^{\tilde{n}-1} \mathscr{C}_{2}(\tilde{n}, b)\left[(g \tilde{n}) \cdot \mathscr{C}_{1}(\tilde{n}, b)\right]^{k-1}
\end{aligned}
$$

wobei wir im letzten Rechenschritt $M$ gegen Unendlich gehen lassen. Das Zwischenergebnis in 3.95 ermöglicht es uns, zusammen mit der Definition von $\mathscr{Z}(b, \tilde{n}, z)$ aus 
(3.50) die Spur der obigen Reihe auszurechnen:

$$
\begin{aligned}
\operatorname{Sp}\left[\sum_{k=1}^{\infty}(-1)^{k}\left(A^{k} C^{-1}\right)\right] & =-(g \tilde{n}) \cdot \sum_{b=1}^{\tilde{n}-1} \mathscr{C}_{2}(\tilde{n}, b)\left\{\sum_{k=0}^{\infty}\left[(-g \tilde{n}) \cdot \mathscr{C}_{1}(\tilde{n}, b)\right]^{k}\right\} \\
& =-(g \tilde{n}) \cdot \sum_{b=1}^{\tilde{n}-1}\left(\mathscr{C}_{2}(\tilde{n}, b) \cdot(\mathscr{Z}(b, \tilde{n}, z))^{-1}\right),
\end{aligned}
$$

falls $\left|(-g \tilde{n}) \cdot \mathscr{C}_{1}(\tilde{n}, b)\right|<1$. Die Relation in $(3.96)$ ergibt sich somit aus dem Wert der geometrischen Reihe innerhalb der geschweiften Klammer. Gemäß der Relation (3.17) können wir nun die Force-Extension berechnen. Der erste Summand in (3.93) ist lediglich die Matrix $C^{-1}$ und führt zum bekannten Marko-Siggia-Term [13, 87. Alle restlichen Reihenglieder enthalten die Matrix $A$ und entsprechen dem crosslinkabhängigen Anteil der Force-Extension-Relation. Daraus schlussfolgern wir mit den Abkürzungen aus (3.89)

$$
\begin{aligned}
& \left.\frac{\langle x(L)\rangle}{L}\right|_{\text {xlink }}=-\frac{1}{8} \cdot \sum_{l=1}^{\infty} q_{l}^{2} \cdot \operatorname{Sp}\left[\sum_{k=1}^{\infty}(-1)^{k}\left(A^{k} C^{-1}\right)\right] \\
& =\left(\frac{\tilde{g} \tilde{T}^{2}}{8 z^{2}}\right) \sum_{b=1}^{\tilde{n}-1}\left(\left(\frac{1}{\tilde{n}}\right)\left(t_{2}(b, \tilde{n})+t_{3}(b, \tilde{n}, z)\right)-\left(\frac{3}{\sqrt{z}}\right) t_{1}(b, \tilde{n}, z)\right)(\mathscr{Z}(b, \tilde{n}, z))^{-1} .
\end{aligned}
$$

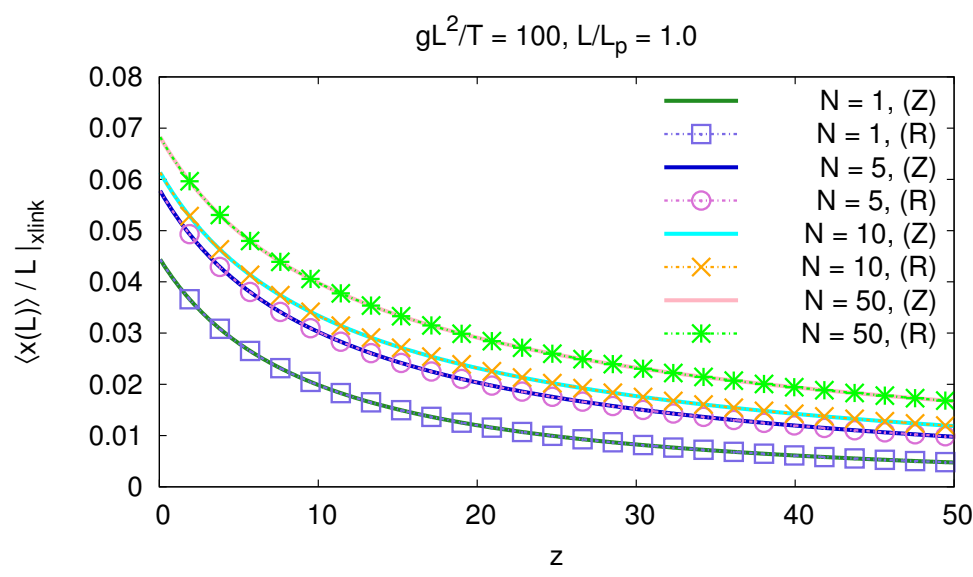

Abbildung 3.8.: Vergleich der Ergebnisse des crosslinkabhängigen Anteils an der ForceExtension $\left.\langle x(L)\rangle \cdot L^{-1}\right|_{\text {xlink }}$, gewonnen aus der Zustandssumme $(\mathrm{Z})$ und mit Hilfe der Reihenentwicklung $(\mathrm{R})$

Mit Abbildung (3.8) wird graphisch die Gleichheit der Ergebnisse aus der Reihenentwicklung (R), (siehe Gleichung (3.97) aus hiesigem Kapitel 3.8) und aus der relativen Zustandssumme (Z), (siehe Gleichung (3.52) aus Kapitel 3.6) überprüft und kann demnach verifiziert werden. Auch hier verwenden wir wie bei Abbildung (3.7) ein Verhältnis von Kontur- zu Persistenzlänge von $L / L_{P}=1.0$. 
Damit haben wir insgesamt drei verschiedene Lösungsmethoden diskutiert, mit denen auf analytischem Wege die Force-Extension-Relation für das genannte Modellsystem berechnet werden kann.

\subsection{Zusammenfassung und Ausblick}

In diesem Kapitel wurde die Force-Extension-Relation für mittels Crosslinks vernetzte, semiflexible Biopolymere, die einer axial an den Kettenenden wirkenden Kraft ausgesetzt sind, präsentiert. Wir konnten feststellen, dass es mehrere Methoden zur Lösung dieses analytischen Problems gibt.

Aus dem Ergebnis kann abgeleitet werden, dass eine Erhöhung der Crosslinkzahl eine Verringerung der transversalen Fluktuationen zur Folge hat und damit zu einer Absenkung der Elastizität bzw. zu einer Erhöhung der Biegesteifigkeit des Modellsystems führt. Dieser Anstieg der Steifigkeit ist jedoch nichtlinear, genauer gesagt wird der Beitrag jedes weiteren, zusätzlichen Crosslinks zur Erhöhung der Steifigkeit immer geringer. Außerdem konnten wir sehen, dass der Crosslink-Anteil an der Force-Extension mit zunehmender Zugkraft immer kleiner wird.

Als eines der nächsten Ziele könnte man in Betracht ziehen, die Force-Extension eines Systems mit ähnlichen Bedingungen zu analysieren. Zum Beispiel könnte man andere Randbedingungen an den Kettenenden einführen 98. Eine sich stellende Frage ist die nach dem Einfluss der Crosslink-Positionen, das heißt, wie würde sich die Force-Extension ändern, falls andere Verknüpfungspunkte für die Crosslinks zugelassen wären.

Zum Beispiel wurde das kollektive Verhalten reversibler Crosslinks unter dem Einfluss der Zugspannung bereits studiert [15]. Ein anderes, ebenfalls interessantes Vorhaben wäre es, die Anzahl der Ketten des Systems schrittweise zu erhöhen. Dies würde einer Betrachtung von Bündeln entsprechen [14]. 


\section{A. Anhang zum Simulationsteil}

In diesem Teil des Anhangs liegen einige zusätzliche Informationen und Ergänzungen zum Simulationsteil vor. Neben den Akzeptanzraten werden unter anderem supplementäre Größen wie die diskrete Krümmung oder ein alternatives Maß zur Messung des Quenched Disorder-Effektes erläutert.

\section{A.1. Akzeptanzraten}

Die in Kapitel 2.10 besprochenen Akzeptanzraten hängen aufgrund des exponentiellen Charakters des Metropolis-Auswahlkriteriums 2.47) stark von den gewählten Systemvorgaben bzw. den Parametern ab, da diese die durch einen Monte-Carlo-Schritt bewirkten Energieänderungen $\triangle E$ wesentlich beeinflussen. Wie groß dieser Einfluss ist, wird anhand der folgenden Diagramme verdeutlicht, in denen die Akzeptanzraten $a(\mu \rightarrow \nu)$ sowohl für die Bend-Moves als auch für die Crosslink-Moves in Abhängigkeit der wählbaren Parameter graphisch wiedergegeben werden.

Wir sind darauf bedacht, zu geringe Akzeptanzraten zu vermeiden. Werden vorgeschlagene Zustandsänderungen zu oft verworfen, so kann dies dazu führen, dass thermische Fluktuationen stark unterdrückt werden und das System als annähernd statisch betrachtet werden kann. Ungünstig gewählte Parameter, die eine geringe Akzeptanz der vorgeschlagenen MC-Schritte nach sich ziehen, würden nur sehr langsame Zustandsänderungen zulassen und die zur Equilibrierung des Systems benötigte Zeit erhöhen.

\section{A.2. Bend- und Crosslink-Moves}

Die Akzeptanzraten $a(\mu \rightarrow \nu)$ der in Kapitel 2.11 beschriebenen Bend-Moves werden als Funktion der Crosslink-Stärke $\alpha$ (siehe Abb. A.1)), der Biegesteifigkeit $\kappa$ (siehe Abb. A.2) und in Abhängigkeit von der Crosslink-Dichte $\rho$ (siehe Abb. (A.3)) gezeigt. Für die Datengenerierung lagen abstandsgleiche Ketten $(\mathcal{R} G r=0)$ ohne eine Excluded Volume-Wechselwirkung vor $(E x-\mathrm{Vol}=0)$.

Es lässt sich ganz allgemein schlussfolgern, dass die Akzeptanzrate mit

- steigender Crosslink-Stärke $\alpha$

- steigender Crosslink-Dichte $\rho$

- zunehmender Biegesteifigkeit $\kappa$

abnimmt.

Offensichtlich weist der Abfall der Akzeptanzrate als Funktion von $\alpha$ einen exponentiel- 
len Charakter auf, wobei dieser mit zunehmender Crosslink-Dichte (siehe Abb. A.1)) sowie mit abnehmender Biegesteifigkeit verstärkt wird. Im Unterschied dazu nimmt die Akzeptanzrate linear mit wachsender Crosslink-Dichte ab, und die negative Steigung wächst mit steigender Crosslink-Stärke $\alpha$ (siehe Abb. A.3)).

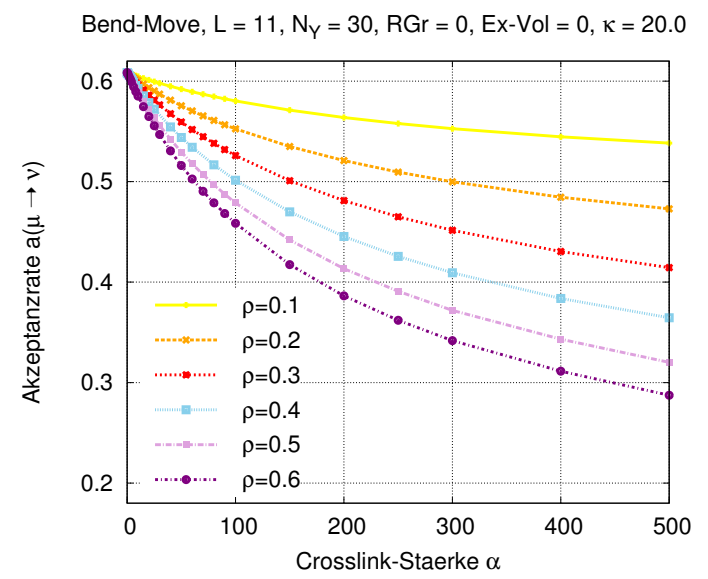

Abbildung A.1.: Bend-Move: $a(\mu \rightarrow \nu)$ in Abhängigkeit von $\alpha$ bei verschiedenen Werten von $\rho$

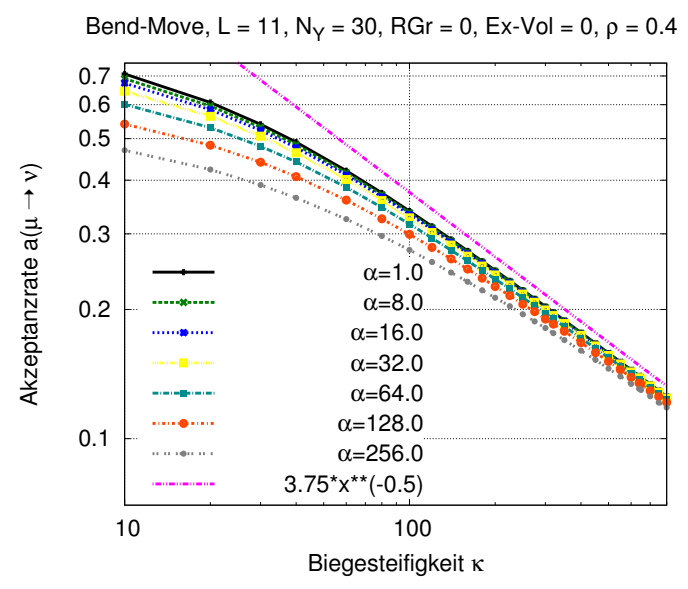

Abbildung A.2.: Bend-Move: $a(\mu \rightarrow \nu)$ in Abhängigkeit von $\kappa$ bei verschiedenen Werten von $\alpha$

Außerdem kann man feststellen, dass der Einfluss der Crosslink-Stärke auf die Akzeptanzrate mit zunehmendem $\kappa$ geringer wird. Weisen die Akzeptanzraten bei kleinen $\kappa$-Werten noch hohe Unterschiede bezüglich $\alpha$ auf, so wird diese Differenz mit Erhöhung der Biegesteifigkeit stetig kleiner und die Kurven mit unterschiedlichen $\alpha$-Werten münden in einen gemeinsamen Verlauf, der eine Proportionalität $a(\mu \rightarrow \nu) \propto \frac{1}{\sqrt{\kappa}}$ offenbart (siehe Abb. A.2).

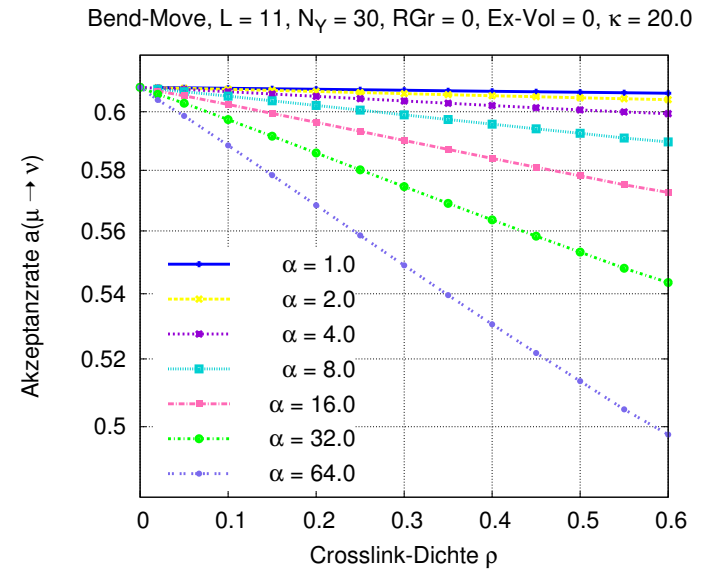

Abbildung A.3.: Bend-Move: $a(\mu \rightarrow \nu)$ in Abhängigkeit von $\rho$ bei verschiedenen Werten von $\alpha$

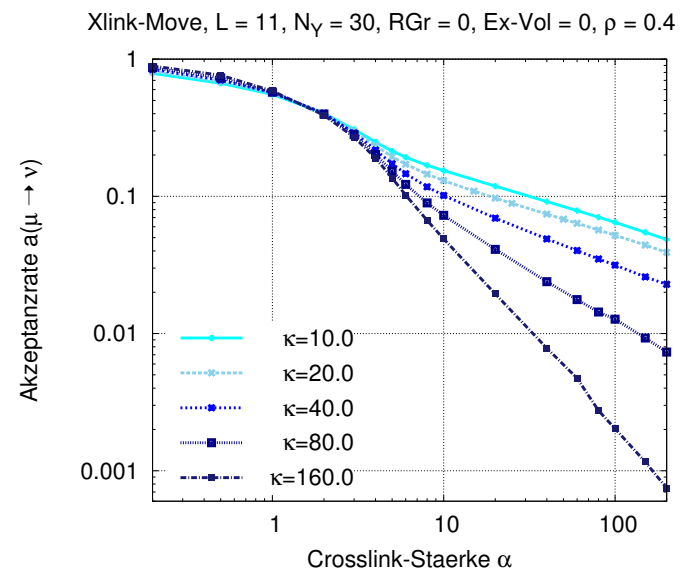

Abbildung A.4.: Crosslink-Move: $a(\mu \rightarrow \nu)$ in Abhängigkeit von $\alpha$ bei verschiedenen Werten von $\kappa$

Mit Hilfe einer einfachen analytischen Abschätzung kann das Verhalten der Bend- 
Move-Akzeptanzraten zumindest in einer einfachen Näherung nachvollzogen werden (siehe Anhang A.3).

Die Charakteristik der Crosslink-Move-Akzeptanzraten ist erwartungsgemäß völlig anders. Auch hier fällt $a(\mu \rightarrow \nu)$ wie bei den Bend-Moves mit zunehmender CrosslinkStärke ab, was anhand der nächsten drei Diagramme (siehe Abbildungen (A.4), (A.5) und (A.6p) gezeigt wird. Untersucht man die Abhängigkeit von $\kappa$, so fällt eine Besonderheit auf: Während die Akzeptanzrate für hinreichend kleines $\alpha$ mit größer werdender Biegesteifigkeit ansteigt, ist für eine genügend hohe Crosslink-Stärke der umgekehrte Trend festzustellen, das heißt $a(\mu \rightarrow \nu)$ fällt mit zunehmendem $\kappa$ ab, und dieser Abfall wird mit wachsendem $\alpha$ umso stärker (siehe Abb. A.6p).

Übersteigt die Crosslink-Stärke einen kritischen Wert von $\alpha \approx 6$, so zeigt sich für die Akzeptanzrate als Funktion von $\alpha$ ein Potenzgesetz (siehe Abb. (A.4)): so fällt $a(\mu \rightarrow \nu)$ umso stärker ab, je höher die Biegesteifigkeit der Ketten ist und wir finden $a(\mu \rightarrow \nu) \propto$ const. $\cdot \alpha^{\gamma(\kappa)}$, mit einem von $\kappa$ abhängigen negativen Exponenten $\gamma(\kappa)$.

Wird $\rho$ bei verschiedenen Werten von $\alpha$ variiert, so bildet sich ein schwach ausgeprägtes Maximum von $a(\mu \rightarrow \nu)$ bei einem Bereich zwischen $\rho \approx 0.2, \ldots, 0.3$ heraus (siehe Abb. (A.5). Steigert man ausgehend von diesem Maximum die Crosslink-Dichte im System, so stellt man fest, dass je nach Federstärke $\alpha$ die Akzeptanzrate für einen CrosslinkMove unterschiedlich stark abfällt. So fällt $a(\mu \rightarrow \nu)$ zum Beispiel für $\alpha=128.0$ zwischen $\rho_{1}=0.25$ und $\rho_{2}=0.6$ um circa 3 Größenordnungen ab.

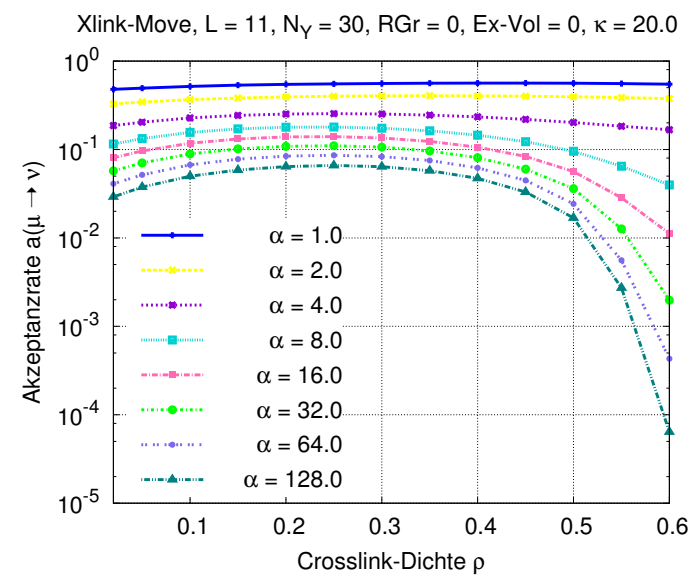

Abbildung A.5.: Crosslink-Move: $a(\mu \rightarrow \nu)$ in Abhängigkeit von $\rho$ bei verschiedenen Werten von $\alpha$

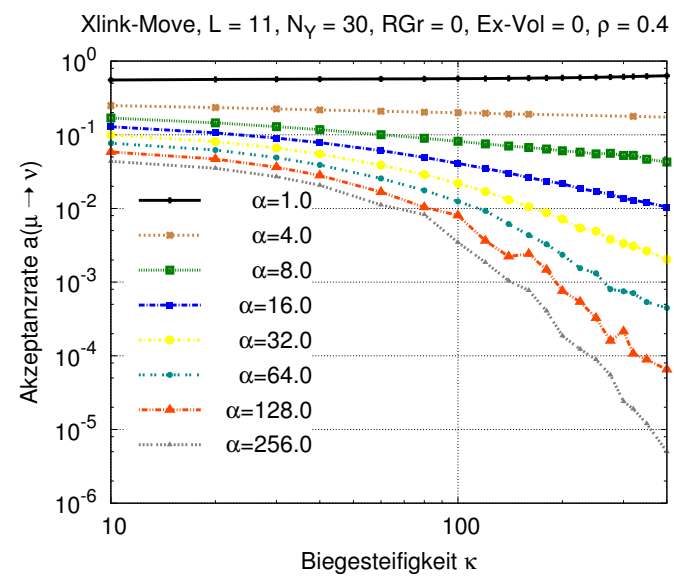

Abbildung A.6.: Crosslink-Move: $a(\mu \rightarrow \nu)$ in Abhängigkeit von $\kappa$ bei verschiedenen Werten von $\alpha$

Prinzipiell kann die Gesetzmäßigkeit abgeleitet werden, dass hohe Werte der Parameter $\alpha, \kappa$ und $\rho$ zu extrem geringen Crosslink-Move-Akzeptanzraten führen, die im Vergleich zu den entsprechenden Bend-Move-Akzeptanzraten um mehrere Größenordnungen kleiner sind. Die harten Crosslinks sind im thermischen Gleichgewicht vermehrt am Kettenende zu finden, wo sie Ketten zusammenziehen und innerhalb von Bündelstrukturen im Mittel kleine Crosslink-Längen aufweisen (siehe Abb. 2.46) in Kapitel 2.22 und Abbildung (2.52) in Kapitel 2.23). Somit besteht bei einem durch den Monte- 
Carlo-Algorithmus vorgeschlagenen Crosslink-Move eine hohe Wahrscheinlichkeit, dass per Zufall ein Crosslink aus der Bündelformation mit niedriger Länge und damit niedriger Crosslink-Energie ausgewählt wird und der zugewiesene neue Gitterplatz zu einer Erhöhung der mittleren Crosslink-Länge und damit zu einer $\alpha$-abhängigen Energieerhöhung $\triangle E$ führen würde. Diese Energieerhöhung würde allerdings nur mit einer geringen Wahrscheinlichkeit gemäß 2.47) akzeptiert werden.

Dies führt dazu, dass die Parameter innerhalb geeigneter Grenzen zu wählen sind. Andernfalls benötigt das System aufgrund der extrem langsamen Equilibierungszeit der Crosslink-Moves eine lange Simulationszeit bis zum Erreichen des thermischen Gleichgewichts.

Eine analytische Abschätzung wie bei den Bend-Moves ist hier schwieriger, da es bei den Crosslink-Moves viel stärker auf die genaue Kenntnis der Segment-Abstände der dem Crosslink-Move zugeordneten Zustände $\mu$ und $\nu$ ankommt. So ist in diesem Fall kein einfaches Modell wie im Falle der Bend-Moves aufstellbar.

\section{A.3. Bend-Move: Analytische Abschätzung der Akzeptanzrate}

In Kapitel A.2 sind die sich aus der Simulation ergebenden Akzeptanzraten für BendMoves $a_{\text {bend }}(\alpha, \kappa, \rho)$ für verschiedene Parameter-Kombinationen von $\alpha, \kappa$ und $\rho$ aufgetragen. Eine einfache analytische Näherung von $a_{\text {bend }}(\alpha, \kappa, \rho)$ lässt sich dafür mit Hilfe der folgenden Annahme realisieren. In unserem Ansatz gehen wir von geraden Ketten aus, das heißt alle Segmente sind unausgelenkt $\left(u_{s, i}=0 \forall i=1, \ldots, N_{Y}\right.$; $s=0,1, \ldots, L)$. Der Kettenabstand zweier benachbarter Ketten wird als konstant angenommen und mit $d \in[0,1]$ bezeichnet.

Das für den Bend-Move ausgewählte Segment habe die Koordinaten $(s, i)$. Dieses wird per gleichverteilter Zufallszahl $\xi$ innerhalb eines Intervalls mit dem Intervallmittelpunkt 0 und der Breite $2 f$ nach oben oder nach unten ausgelenkt, d. h. $\xi \in[-f,+f]$. Mit $E_{\kappa}^{v}$, $E_{\kappa}^{n}$ werden die lokalen Biegeenergien und mit $E_{\alpha}^{v}, E_{\alpha}^{n}$ die lokalen Crosslink-Energien des Systems vor (Index $v$ ) bzw. nach (Index $n$ ) einem Bend-Move bezeichnet. Mit der Ausführung eines Bend-Moves geht folgende Energieänderung einher:

$$
\begin{aligned}
\triangle E(\xi) & =\left(E_{\kappa}^{n}+E_{\alpha}^{n}\right)-\left(E_{\kappa}^{v}+E_{\alpha}^{v}\right) \\
& =6 \kappa \xi^{2}+\alpha\left[\mathbb{1}_{\mathcal{C}}((s, i+1))\left(\xi^{2}-2 d \xi\right)+\mathbb{1}_{\mathcal{C}}((s, i))\left(\xi^{2}+2 d \xi\right)\right] .
\end{aligned}
$$

Die charakteristischen Funktionen berücksichtigen die Möglichkeit einer Verknüpfung des betreffenden Segments $(s, i)$ mit bis zu zwei Crosslinks, was durch Belegungen der Gitterplätze $(s, i+1)$ und $(s, i)$ durch Crosslinks möglich ist. Der besseren Übersicht halber definieren wir

$$
\mathrm{p}:=\mathbb{1}_{\mathcal{C}}(s, i+1)+\mathbb{1}_{\mathcal{C}}((s, i)) \quad, \quad \mathrm{m}:=\mathbb{1}_{\mathcal{C}}((s, i+1))-\mathbb{1}_{\mathcal{C}}((s, i))
$$

und geben die Werte der vier möglichen Fälle für Crosslink-Belegungen an:

1. 0 Crosslinks auf den Gitterplätzen $(s, i+1)$ und $(s, i) \Rightarrow \mathrm{p}=0, \mathrm{~m}=0$ 
2. 1 Crosslink auf dem Gitterplatz $(s, i+1) \Rightarrow \mathrm{p}=1, \mathrm{~m}=+1$

3. 1 Crosslink auf dem Gitterplatz $(s, i) \Rightarrow \mathrm{p}=1, \mathrm{~m}=-1$

4. 2 Crosslinks auf den Gitterplätzen $(s, i+1)$ und $(s, i) \Rightarrow \mathrm{p}=2, \mathrm{~m}=0$, wobei die beiden Fälle (2.) und (3.) aus Symmetriegründen äquivalent sind.

Die Energiedifferenz $\triangle E(\xi)$ ist also im Rahmen dieses Modells nur dann vom Kettenabstand $d$ abhängig, wenn genau ein Crosslink mit dem auszulenkenden Segment $(s, i)$ verknüpft ist (siehe Gl. A.1P). Zur Bestimmung der Akzeptanzrate, basierend auf den oben genannten Vereinfachungen, ist das normierte Integral

$$
\mathcal{I}(\alpha, \kappa)=\frac{1}{(2 f)} \cdot \int_{-f}^{f} \exp (-\beta \triangle E(\xi)) \mathrm{d} \xi \quad \text { mit } \quad a_{\text {bend }}(\alpha, \kappa)=\min \{1, \mathcal{I}(\alpha, \kappa)\}
$$

auszuwerten. Schließlich erhalten wir für $\mathcal{I}(\alpha, \kappa)$ in Abhängigkeit der Parameter $\alpha, \kappa$ und unter Berücksichtung der oben genannten Fälle

$$
\mathcal{I}(\alpha, \kappa)=\frac{\sqrt{\pi} \exp \left(\frac{(\alpha \mathrm{m} d)^{2}}{\mathrm{p} \alpha+6 \kappa}\right)\left[\operatorname{erf}\left(\frac{\alpha(\mathrm{p} f-\mathrm{m} d)+6 f \kappa}{\sqrt{\mathrm{p} \alpha+6 \kappa}}\right)+\operatorname{erf}\left(\frac{\alpha(\mathrm{p} f+\mathrm{m} d)+6 f \kappa}{\sqrt{\mathrm{p} \alpha+6 \kappa}}\right)\right]}{2 \sqrt{\mathrm{p} \alpha+6 \kappa}} .
$$

Machen wir eine weitere Vereinfachung und setzen den Kettenabstand $d$ gleich Null, so vereinfacht sich A.4 zu

$$
\left.\mathcal{I}(\alpha, \kappa)\right|_{d=0}=\frac{\sqrt{\pi} \operatorname{erf}(f \sqrt{\alpha \mathrm{p}+6 \kappa})}{(\sqrt{\alpha \mathrm{p}+6 \kappa})} .
$$

Berücksichtigen wir, dass die Wahrscheinlichkeit für die Belegung eines Gitterplatzes mit einem Crosslink der Crosslink-Dichte $\rho$ entspricht, so können wir die Akzeptanzrate wie folgt schreiben:

$$
\begin{aligned}
a_{\text {bend }}(\alpha, \kappa, \rho) & =\left.(1-\rho)^{2} \cdot a_{\text {bend }}(\alpha, \kappa)\right|_{\mathrm{p}=0, \mathrm{~m}=0} \\
& +\left.2(1-\rho) \rho \cdot a_{\text {bend }}(\alpha, \kappa)\right|_{\mathrm{p}=1, \mathrm{~m}= \pm 1}+\left.\rho^{2} \cdot a_{\text {bend }}(\alpha, \kappa)\right|_{\mathrm{p}=2, \mathrm{~m}=0} .
\end{aligned}
$$

Die Abbildungen (A.7) und A.8) zeigen exemplarisch die Akzeptanzrate $a_{\text {bend }}(\alpha, \kappa, \rho)$ für $d=0$. Obwohl es sich hierbei lediglich um eine relativ einfache Abschätzung handelt, erkennt man dennoch ein vergleichbares Verhalten wie bei den gemessenen numerischen Akzeptanzraten (vgl. Abb. A.1) und (A.2)).

Dass die analytisch berechneten Werte der Akzeptanzraten insgesamt geringer ausfallen, ist nachvollziehbar. Denn eine Segmentauslenkung aus einer exakt gerade ausgerichteten Kette heraus führt immer zu einer stärkeren Krümmung und damit zu einer Energieerhöhung. In Wirklichkeit verfügen die thermisch equilibrierten Ketten über einen von Null verschiedenen Krümmungsgrad, der in Abhängigkeit der entsprechenden Parameter variiert (siehe nächstes Kapitel A.4). Diese Tatsache alleine führt folglich dazu, dass in der realen Simulation häufig Bend-Moves vorgeschlagen werden, die vom Algorithmus immer akzeptiert werden, da sie zu einer geringeren Kettenkrümmung und damit zu einer Energieerniedrigung führen. Ein weiterer Grund für Abweichungen zwischen Simulation und analytischer Abschätzung liegt im von Null verschiedenen, variablen Kettenabstand. 


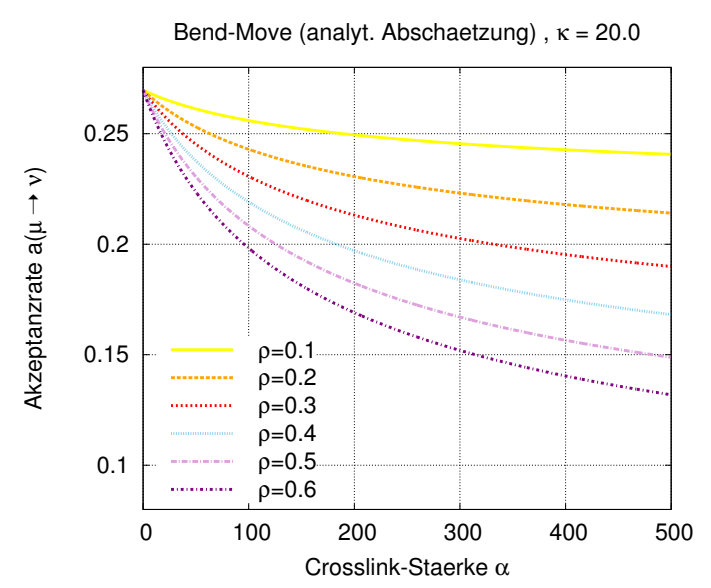

Abbildung A.7.: Akzeptanzrate $a_{\text {bend }}$ in Abhängigkeit von $\alpha$ bei verschiedenen Werten von $\rho$

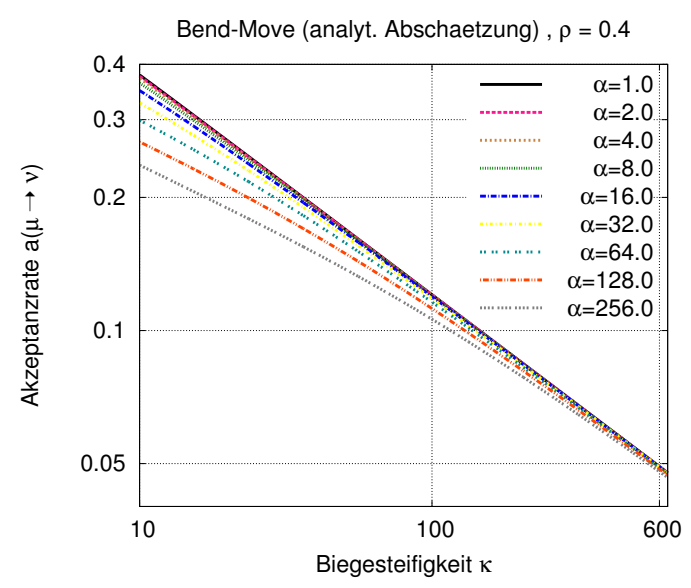

Abbildung A.8.: Akzeptanzrate $a_{\text {bend }}$ in Abhängigkeit von $\kappa$ bei verschiedenen Werten von $\alpha$

\section{A.4. Diskrete Krümmung $c(s)$}

Wie bereits in Kapitel 2.5 beschrieben, werden die Polymerketten mit Hilfe von diskreten Segmentkoordinaten $(s, i)$ charakterisiert. Demzufolge kann daraus unmittelbar die diskretisierte Konformation und damit auch die diskrete Krümmung $c(s)$ abgeleitet werden. Mit der mittleren diskreten Krümmung bezeichnen wir per Definition die thermische Mittelung der lokalen Krümmungen, die durch die Auslenkungswerte dreier benachbarter Segmente beschrieben wird (siehe Gleichung (2.18)):

$$
c(s) \equiv\left\langle c_{s}\right\rangle:=\lim _{t \rightarrow \infty} \frac{1}{t} \int_{0}^{t} c_{s}\left(t^{\prime}\right) \mathrm{d} t^{\prime},
$$

mit dem Mittelwert der lokalen Krümmung am Segment $s$

$$
c_{s}\left(t^{\prime}\right):=\frac{1}{N_{Y}} \cdot \sum_{i=1}^{N_{Y}}\left(u_{s-1, i}\left(t^{\prime}\right)-2 u_{s, i}\left(t^{\prime}\right)+u_{s+1, i}\left(t^{\prime}\right)\right)^{2} \quad \text { und } \quad s=1,2, \ldots,(L-1) .
$$

Wir untersuchen also segmentweise die Krümmungswerte der Ketten, wobei jeweils über alle $N_{Y}$ Ketten gemittelt wird. Für die beiden äußersten Segmente $s \in\{0, L\}$ ist nach dieser Definition trivialerweise kein Wert definiert, so dass diese hier unberücksichtigt bleiben.

Die beiden Diagramme A.9 und A.10 zeigen den Verlauf der diskreten Krümmung für unterschiedliche Werte von $\rho$ bzw. $\kappa$, während die jeweils verbliebenen Parameter konstant gehalten wurden. Erwartungsgemäß führt eine geringe Anzahl von Crosslinks nur zu geringen Krümmungsschwankungen entlang des Segment-Indexes $s$. Bei der Erhöhung von $\rho$ kann man feststellen, dass sich die Krümmung im Bereich des festen Endes erhöht. Dies ist auch verständlich, denn mit steigender Crosslinkzahl tritt verstärkt Bündelbildung auf, die dazu führt, dass die Ketten im genannten Übergangsbereich (zwischen den Segmenten $s=1$ und $s=6$ ) stärker gekrümmt werden. Im 


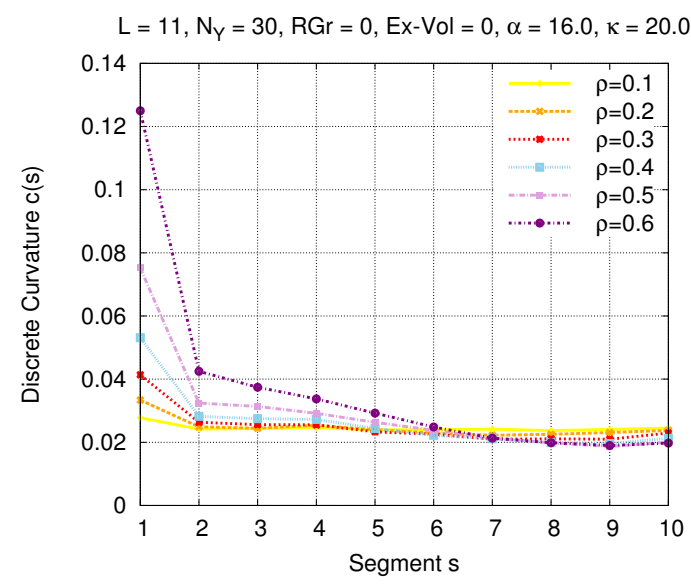

Abbildung A.9.: Mittlere Krümmung $c(s)$ als Funktion von $s$ für verschiedene Werte von $\rho$

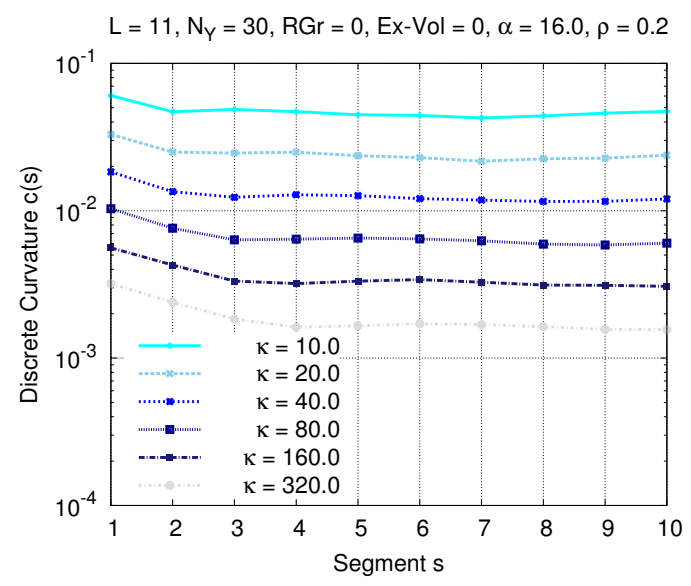

Abbildung A.10.: Mittlere Krümmung $c(s)$ als Funktion von $s$ für verschiedene Werte von $\kappa$

Bereich des freien Endes (hier zwischen den Segment-Indizes $s=6$ und $s=L$ ), weisen die Ketten im gebündelten Zustand in etwa die selbe Krümmung auf.

Einen verständlicherweise erheblichen und deutlich höheren Einfluss auf die KettenKrümmung hat die Variation der Biegesteifigkeit $\kappa$ (siehe Abb. A.10). Hierbei wird sehr klar der Zusammenhang zwischen einer höheren Biegesteifigkeit und einer Verringerung der Krümmung entlang der gesamten Kettenkontur deutlich.

\section{A.5. Richtungskorrelation $C_{t}(s)$ mit Excluded Volume-Wechselwirkung}

Wenn die Ketten sich nicht mehr kreuzen bzw. durchdringen können, stellt dies eine enorme Einschränkung ihrer Bewegungsfreiheit dar. Diese Eigenschaft wird mit Hilfe der Excluded Volume-Wechselwirkung $(E x$ - Vol $=1)$ realisiert (siehe Kapitel 2.8). Die Fluktuationen werden dabei stark unterdrückt, da sich die Ketten gegenseitig behindern. Im zeitlichen Mittel ist deshalb die Bewegung der Bond-Vektoren weg von der axialen Ausrichtung weniger wahrscheinlich, was zu einer deutlichen Erhöhung der Richtungskorrelation führt (siehe Abb. A.11)). Diese Tatsache wird durch den Vergleich mit Abbildung (2.36) in Kapitel 2.19 verdeutlicht, in der der Fall ohne Excluded Volume-Wechselwirkung $(E x-V o l=0)$ aufgetragen ist.

Abbildung (A.12 zeigt den Einfluss der Excluded Volume-Wechselwirkung bei Erhöhung der Crosslink-Dichte $\rho$. Mit steigendem Crosslink-Anteil und einer damit verbundenen verstärkten Verknüpfung der Ketten erfolgt Bündelbildung. Daraus folgt, dass durch die zunehmende Kopplung der Ketten miteinander verstärkt kollektive Bewegungen möglich sind, so dass die Ketten vermehrt im Gleichklang miteinander und nicht gegeneinander schwingen, wie im Falle crosslinkfreier Ketten. Dies spiegelt sich letztendlich in einem niedrigeren Wert von $\left.C_{t}(s)\right|_{\rho>0}$ im Vergleich zu $\left.C_{t}(s)\right|_{\rho=0}$ wider. 


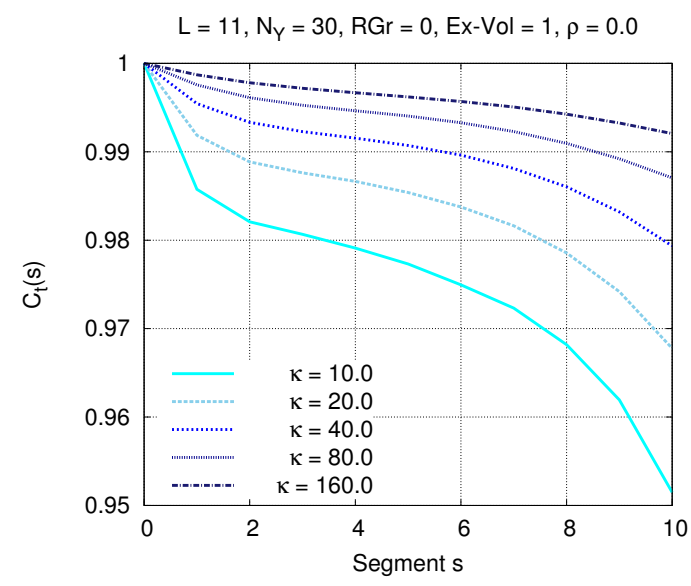

Abbildung A.11.: $C_{t}(s)$ (mit $(\mathcal{R} G r=1)$ ), aufgetragen über $s$ bei verschiedenen Werten von $\kappa$

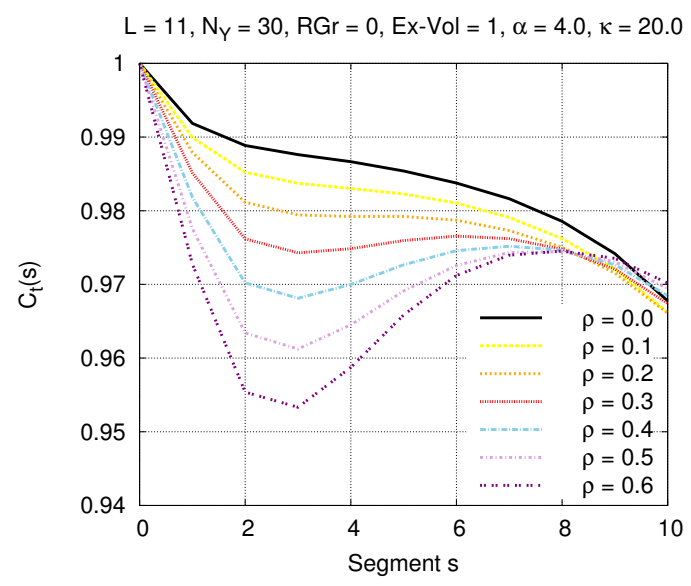

Abbildung A.12.: $C_{t}(s)(\operatorname{mit}(\mathcal{R} G r=1))$, aufgetragen über $s$ bei verschiedenen Werten von $\rho$

\section{A.6. Winkelverteilung unter dem Einfluss von Crosslinks}

Bisher hatten wir lediglich die Winkelverteilung von wechselwirkungsfreien Ketten betrachtet (siehe Kaptiel 2.21). In diesem Kapitel wird kurz erläutert, wie sich das Hinzufügen von Crosslinks auf die Wahrscheinlichkeitsverteilung $P\left(s, \theta_{s}\right)$ bei einem beliebig gewählten, aber festen Segment-Index $s=8$ auswirkt. Wir berücksichtigen dabei wieder den Quenched Disorder-Mittelwert, das heißt die Mittelung von $P\left(s, \theta_{s}\right)$ bei $r_{\max }$ verschiedenen Grafting-Positionen $(\mathcal{R} G r=1)$ :

$$
\left.P\left(s, \theta_{s}\right)\right|_{\mathrm{qd}}:=\frac{1}{r_{\max }} \cdot \sum_{r=1}^{r_{\max }} P_{r}\left(s, \theta_{s}\right) .
$$

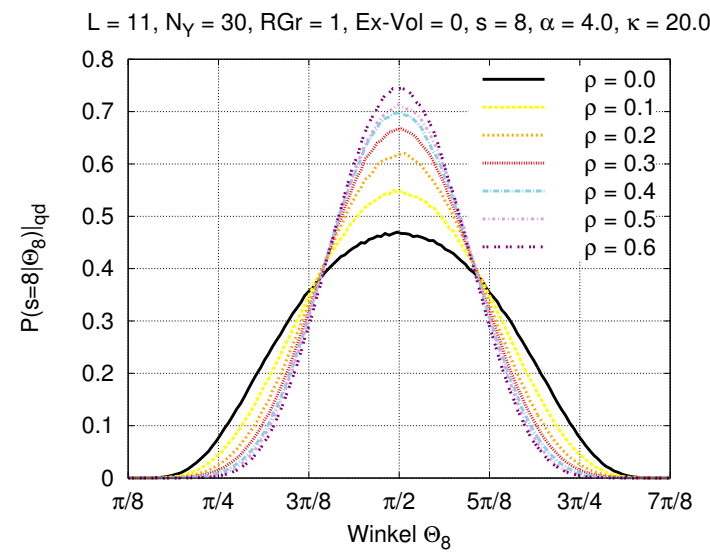

Abbildung A.13.: $\left.P\left(8, \theta_{8}\right)\right|_{\text {qd }}$ bei festem $\alpha$ für verschiedene Werte von $\rho$

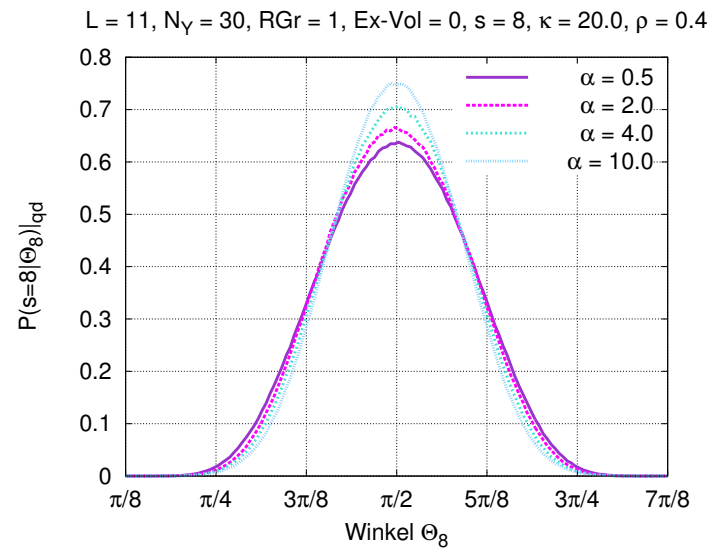

Abbildung A.14.: $\left.P\left(8, \theta_{8}\right)\right|_{\text {qd }}$ bei festem $\rho$ für verschiedene Werte von $\alpha$ 
Es wird deutlich (siehe Abb. A.13), dass auch Polymerketten, die durch Crosslinks vernetzt sind, Fluktuationsbewegungen ausführen, die zu einer gaußförmigen Winkelverteilung führen. Dabei sind die Schwankungen umso geringer, je höher der CrosslinkAnteil des Systems ist. Offensichtlich führen die durch die Crosslinks entstandenen Polymerbündel wie eine freie Kette im Kollektiv thermische Bewegungen aus.

Die Abhängigkeit der thermischen Fluktuationen von $\alpha$ ist hingegen geringer (siehe Abb. A.14). Durch eine höhere Crosslinksstärke $\alpha$ veringert sich die Schwankung nur geringfügig.

\section{A.7. Alternatives Maß zur Messung des Quenched Disorder-Effektes}

In Kapitel 2.16 haben wir eine Methode vorgestellt, eingeforene Zustände mit Hilfe der Größe $a(t)$ quantitativ zu erfassen. Der Quenched Disorder-Effekt kann aber auch mit Hilfe der mittleren quadratischen Verschiebung der Segmente am Kettenende bzw. mit deren Standardabweichung (siehe Gleichungen (2.53) und (2.54) verdeutlicht werden.

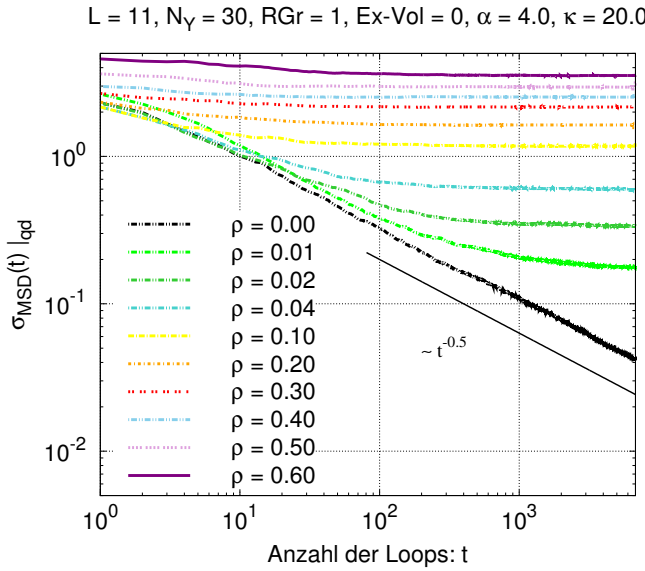

Abbildung A.15.: $\left.\sigma_{\mathrm{MSD}}(t)\right|_{\mathrm{qd}}$ für verschiedene Crosslink-Dichten $\rho$

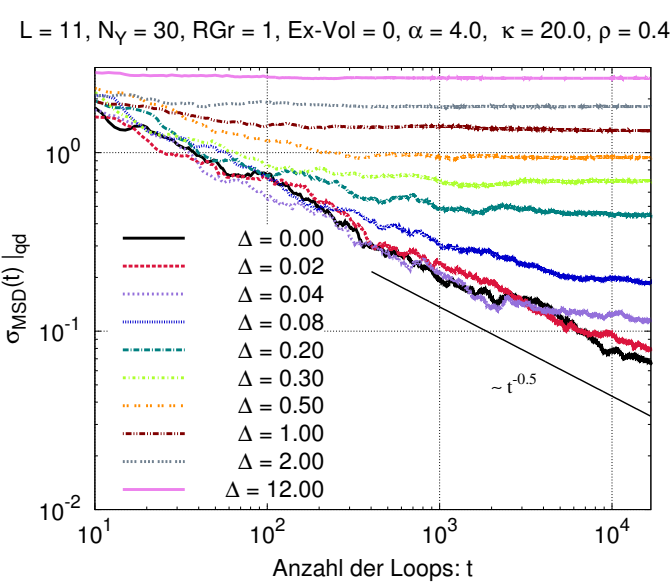

Abbildung A.16.: $\left.\sigma_{\mathrm{MSD}}(t)\right|_{\mathrm{qd}}$ für verschiedene Intervallbreiten $\Delta$

In den beiden Abbildungen (A.15) und A.16) werden die Quenched Disorder-Mittelwerte von $\sigma_{\mathrm{MSD}}(t)$ zum Einen für verschiedene Crosslink-Dichten $\rho$, zum Anderen für verschiedene Intervallbreiten $\Delta$ in Abhängigkeit der Zeit $t$ gezeigt. Nur bei unabhängigen $(\rho=0)$ oder äquidistant aufgepfropften Ketten $(\Delta=0)$ kann man für die Standardabweichung der mittleren quadratischen Abweichung die Gesetzmäßigkeit $\left.\sigma_{\mathrm{MSD}}(t)\right|_{\mathrm{qd}} \propto 1 / \sqrt{t}$ nachweisen. Andernfalls wird sich $\left.\sigma_{\mathrm{MSD}}(t)\right|_{\mathrm{qd}}$ im Grenzfall $t \rightarrow \infty$ auf konstante Plateauwerte einpendeln. Je stärker die zeitlich gemittelten Kettenkonformationen von der Ruhelage abweichen, desto höher sind die Plateauwerte. Auch hier ist erkennbar, dass sowohl die Erhöhung von $\Delta$ bei gegebener, von Null verschiedener Crosslink-Dichte als auch die Erhöhung von $\rho$ bei gegebener maximaler Grafting-Unordnung zu einem vergleichbaren Quenched Disorder-Effekt führt, in 
Übereinstimmung mit den Ergebnissen in Kapitel 2.16.

Hierbei wird sichtbar, dass die Größe $\left.\sigma_{\mathrm{MSD}}(t)\right|_{\mathrm{qd}}$ in vergleichbarer Weise zur Bestimmung eines Quenched Disorder-Effektes geeignet ist. 


\section{B. Anhang zum analytischen Teil}

Im Anhang werden einige Rechenschritte erläutert, die im Rahmen der analytischen Berechnung gebraucht wurden. Um den Haupttext übersichtlicher zu gestalten, sind bestimmte Berechnungen und Ergänzungen in den Anhang verschoben worden.

\section{B.1. Der Erwartungswert von $J_{l}^{2}$ :}

Den Mittel- oder Erwartungswert von $J_{l}^{2}$ bestimmen wir mit Hilfe der Auswertung von mehrdimensionalen Gaußschen Integralen [44. Wir erhalten für den Erwartungswert von $\Gamma_{l}^{2}$ den folgenden Ausdruck:

$$
\left\langle\Gamma_{l}^{2}\right\rangle=\frac{\int \mathrm{d}^{2 M} \Gamma\left(\Gamma_{l}\right)^{2} e^{-\beta \mathcal{H}[\boldsymbol{\Gamma}]}}{\int \mathrm{d}^{2 M} \Gamma e^{-\beta \mathcal{H}[\boldsymbol{\Gamma}]}} .
$$

\section{Zur Force-Extension im Falle $(N=1)$ :}

Wir berechnen in Kapitel 3.3 die Force-Extension für ein System, das aus zwei Weakly Bending Wormlike Chains und einem bei $s_{1}=L / 2$ platzierten Crosslink besteht. Dabei befinden sich die Kettenenden beider Polymere auf der $x$-Achse, das bedeutet es gilt $\left|y_{1}(s)-y_{2}(s)\right|=0$ für $s \in\{0, L\}$. Damit lässt sich der Hamiltonian im Exponenten als quadratische Form schreiben.

Der Nenner von (B.1) lässt sich direkt lösen, indem wir das $2 M$-dimensionale Gaußsche Integral verwenden [44]:

$$
\int \mathrm{d}^{2 M} \Gamma e^{-\frac{1}{2} \Gamma^{T} G \Gamma}=(2 \pi)^{M} e^{-\frac{1}{2} S p \ln G} .
$$

Zur Bestimmung des Integrals im Zähler gehen wir vom obigen Integral aus und führen einen linearen Term samt Hilfsvektor $\rho$ in den Exponenten ein, so dass wir mittels zweifacher Differentiation nach der Größe $\rho_{l}$ das folgende Resultat erhalten 444:

$$
\begin{gathered}
\int \mathrm{d}^{2 M} \Gamma\left(\Gamma_{l}\right)^{2} e^{-\frac{1}{2} \Gamma^{T} G \Gamma}=\left.\frac{\partial^{2}}{\partial \rho_{l}^{2}} \int \mathrm{d}^{2 M} \Gamma e^{-\frac{1}{2} \Gamma^{T} G \Gamma+\rho^{T} \Gamma}\right|_{\rho=0} \\
=\left.(2 \pi)^{M} e^{-\frac{1}{2} S p \ln G} \frac{\partial^{2}}{\partial \rho_{l}^{2}} e^{\frac{1}{2} \rho^{T} G^{-1} \rho}\right|_{\rho=0}=(2 \pi)^{M} e^{-\frac{1}{2} S p \ln G} \cdot g_{l, l}^{-1} .
\end{gathered}
$$

Mit diesen beiden Zwischenresultaten folgt schießlich unmittelbar das Ergebnis

$$
\left.\left\langle J_{l}^{2}\right\rangle\right|_{d=0}=\left.\left\langle\Gamma_{2 l-1}^{2}\right\rangle\right|_{d=0}=g_{2 l-1,2 l-1}^{-1} .
$$




\section{B.2. Erläuterungen zu $(N=1)$ Crosslinks}

Ausgehend vom Hamiltonian (3.7) ergibt sich durch Anwendung der Normalmoden

$$
\begin{gathered}
\left(\frac{\partial^{2} y_{1}(s)}{\partial s^{2}}\right)^{2}=\sum_{l=1}^{\infty} \sum_{m=1}^{\infty} J_{l} J_{m} q_{l}^{2} q_{m}^{2} \sin \left(q_{l} s\right) \sin \left(q_{m} s\right) \\
\left(\frac{\partial y_{1}(s)}{\partial s}\right)^{2}=\sum_{l=1}^{\infty} \sum_{m=1}^{\infty} J_{l} J_{m} q_{l} q_{m} \cos \left(q_{l} s\right) \cos \left(q_{m} s\right),
\end{gathered}
$$

und aufgrund der Orthogonalitätsrelation

$$
\int_{0}^{L} d s \sin \left(q_{l} s\right) \sin \left(q_{m} s\right)=0 \quad, \quad \int_{0}^{L} d s \cos \left(q_{l} s\right) \cos \left(q_{m} s\right)=0 \quad \text { für } \quad l \neq m
$$

fallen die Integrale mit unterschiedlichen Modenzahlen $(l \neq m)$ weg, so dass wir lediglich bei identischen Indizes einen nicht verschwindenden Beitrag erhalten:

$$
\int_{0}^{L} \sin ^{2}\left(q_{l} s\right) d s=\frac{L}{2}-\underbrace{\frac{\sin \left(2 q_{l} L\right)}{4 q_{l}}}_{=0}, \quad \int_{0}^{L} \cos ^{2}\left(q_{l} s\right) d s=\frac{L}{2}+\underbrace{\frac{\sin \left(2 q_{l} L\right)}{4 q_{l}}}_{=0} .
$$

Daraus folgen die folgenden Vereinfachungen:

$$
\begin{gathered}
\frac{\kappa}{2} \int_{0}^{L} d s\left(\frac{\partial^{2} y_{1}(s)}{\partial s^{2}}\right)^{2}=\frac{\kappa}{2} \int_{0}^{L} \sum_{l=1}^{\infty} q_{l}^{4} J_{l}^{2} \sin ^{2}\left(q_{l} s\right) d s=\frac{\kappa L}{4} \sum_{l=1}^{\infty} q_{l}^{4} J_{l}^{2} \\
\frac{f}{2} \int_{0}^{L} d s\left(\frac{\partial y_{1}(s)}{\partial s}\right)^{2}=\frac{f}{2} \int_{0}^{L} \sum_{l=1}^{\infty} q_{l}^{2} J_{l}^{2} \cos ^{2}\left(q_{l} s\right) d s=\frac{f L}{4} \sum_{l=1}^{\infty} q_{l}^{2} J_{l}^{2} .
\end{gathered}
$$

\section{B.3. Berechnung des Matrixelements $\tilde{p}_{m n}$}

Mit Hilfe der Partialsumme einer geometrischen Reihe können wir die Matrixelemente von $\tilde{P}$ (siehe (3.16) ) bestimmen. Wir finden für $(m-n) \notin 2 \tilde{n} \mathbb{Z} \wedge(m+n) \notin 2 \tilde{n} \mathbb{Z}$

$$
\begin{aligned}
& \tilde{p}_{m n}=g \sum_{l=1}^{N} \sin \left(\frac{l m \pi}{\tilde{n}}\right) \cdot \sin \left(\frac{\ln \pi}{\tilde{n}}\right)=\frac{g}{2} \sum_{l=1}^{N}\left(\cos \left(\frac{(m-n) l \pi}{\tilde{n}}\right)-\cos \left(\frac{(m+n) l \pi}{\tilde{n}}\right)\right) \\
& =\frac{g}{4} \sum_{l=1}^{N}\left(e^{i\left(\frac{(m-n) l \pi}{\tilde{n}}\right)}+e^{-i\left(\frac{(m-n) l \pi}{\tilde{n}}\right)}-e^{i\left(\frac{(m+n) l \pi}{\tilde{n}}\right)}-e^{-i\left(\frac{(m+n) l \pi}{\tilde{n}}\right)}\right) \\
& =\frac{g}{4} \sum_{j=1}^{2}\left\{\frac{(-1)^{j+1}\left[1+e^{i \pi\left(m+(-1)^{j} n\right)}-e^{\frac{i \pi\left(m+(-1)^{j} n\right)}{\tilde{n}}}\left(e^{-i \pi\left(m+(-1)^{j} n\right)}+1\right)\right]}{\left(-1+e^{\frac{i \pi\left(m+(-1)^{j} n\right)}{\tilde{n}}}\right)}\right\} .
\end{aligned}
$$

Man erkennt direkt, dass bei ungeraden Summen (und damit auch ungeraden Differenzen) der Indizes $m, n$ die Matrixelemente verschwinden, das heißt, es gilt

$$
\tilde{p}_{m n}=0 \quad, \text { wenn }(m \pm n) \quad \bmod 2=1 \wedge(m \pm n) \notin 2 \tilde{n} \mathbb{Z} .
$$


Für gerade Summen bzw. Differenzen von $m, n$ können wir allgemein schreiben

$$
\begin{aligned}
\tilde{p}_{m n} & =\frac{g\left(1-e^{\frac{i \pi(m-n)}{\tilde{n}}}\right)\left(-1+e^{-\frac{i \pi(m-n)}{\tilde{n}}}\right)}{1-\cos \left(\frac{\pi(m-n)}{\tilde{n}}\right)}-\frac{g\left(1-e^{\frac{i \pi(m+n)}{\tilde{n}}}\right)\left(-1+e^{-\frac{i \pi(m+n)}{\tilde{n}}}\right)}{1-\cos \left(\frac{\pi(m+n)}{\tilde{n}}\right)} \\
& =-2 g \cdot \frac{1-\cos \left(\frac{\pi(m-n)}{\tilde{n}}\right)}{1-\cos \left(\frac{\pi(m-n)}{\tilde{n}}\right)}+2 g \cdot \frac{1-\cos \left(\frac{\pi(m+n)}{\tilde{n}}\right)}{1-\cos \left(\frac{\pi(m+n)}{\tilde{n}}\right)}=0, \\
& \text { wenn }(m \pm n) \quad \bmod 2=0 \wedge(m \pm n) \notin 2 \tilde{n} \mathbb{Z}
\end{aligned}
$$

und sehen anhand von (B.10) und (B.11), dass die Matrixelemente stets verschwinden, sofern die Bedingung $(m \pm n) \notin 2 \tilde{n} \mathbb{Z}$ gegeben ist.

In den anderen Fällen ergibt sich dagegen

$$
\tilde{p}_{m n}= \begin{cases}+\left(\frac{g \tilde{n}}{2}\right) & , \text { wenn }(m-n) \in 2 \tilde{n} \cdot \mathbb{Z} \wedge(m+n) \notin 2 \tilde{n} \cdot \mathbb{Z} \\ -\left(\frac{g \tilde{n}}{2}\right) & , \text { wenn }(m+n) \in 2 \tilde{n} \cdot \mathbb{Z} \wedge(m-n) \notin 2 \tilde{n} \cdot \mathbb{Z} . \\ 0 & , \text { wenn }(m-n) \in 2 \tilde{n} \cdot \mathbb{Z} \wedge(m+n) \in 2 \tilde{n} \cdot \mathbb{Z}\end{cases}
$$

Somit wird erkennbar, dass die Matrix $\tilde{P}$ periodisch in den Indizes $m$ und $n$ mit der Periode $2 \tilde{n}$ ist. Aufgrund der damit verbundenen Matrixstruktur ist eine Darstellung mit Hilfe von $2 \tilde{n} \times 2 \tilde{n}$-Blockmatrizen sinnvoll. Zur Veranschaulichung nehmen wir hier explizit den Fall $N=3$ Crosslinks. Mit der quadratischen Blockmatrix

$$
\hat{B}:=\left(\frac{g \tilde{n}}{2}\right) \cdot\left(\begin{array}{cccccccc}
+1 & 0 & 0 & 0 & 0 & 0 & -1 & 0 \\
0 & +1 & 0 & 0 & 0 & -1 & 0 & 0 \\
0 & 0 & +1 & 0 & -1 & 0 & 0 & 0 \\
0 & 0 & 0 & 0 & 0 & 0 & 0 & 0 \\
0 & 0 & -1 & 0 & +1 & 0 & 0 & 0 \\
0 & -1 & 0 & 0 & 0 & +1 & 0 & 0 \\
-1 & 0 & 0 & 0 & 0 & 0 & +1 & 0 \\
0 & 0 & 0 & 0 & 0 & 0 & 0 & 0
\end{array}\right) \in \mathcal{M}(2 \tilde{n} \times 2 \tilde{n}, \mathbb{R})
$$

können wir schließlich die Periodizität der quadratischen $2 M \tilde{n} \times 2 M \tilde{n}$-Matrix $\tilde{P}$ verdeutlichen. Die Matrix $\tilde{P}$ enthält $M^{2}$-mal die Matrix $\hat{B}$ :

$$
\tilde{P}=\left(\begin{array}{ccc}
\hat{B} & \ldots & \hat{B} \\
\vdots & \ddots & \vdots \\
\hat{B} & \ldots & \hat{B}
\end{array}\right)
$$

\section{B.4. Angewandte Eigenschaften des Kronecker-Produktes}

In Kapitel 3.5 gebrauchen wir ein paar Eigenschaften des Kronecker-Produktes. Seien die Matrizen $M_{1} \in \mathcal{M}(m \times n, \mathbb{R}), M_{2} \in \mathcal{M}(r \times s, \mathbb{R}), M_{3} \in \mathcal{M}(n \times p, \mathbb{R})$ und $M_{4} \in \mathcal{M}(s \times t, \mathbb{R})$ gegeben. Dann gilt $16,106,110$

$$
\left(M_{1} \otimes M_{2}\right)\left(M_{3} \otimes M_{4}\right)=M_{1} M_{3} \otimes M_{2} M_{4} \in \mathcal{M}(m r \times p t, \mathbb{R}) .
$$


Des Weiteren gilt für die Spur des Kronecker-Produktes zweier quadratischer Matrizen $N_{1} \in \mathcal{M}(m \times m, \mathbb{R})$ und $N_{2} \in \mathcal{M}(n \times n, \mathbb{R})$

$$
\operatorname{Sp}\left(N_{1} \otimes N_{2}\right)=\operatorname{Sp}\left(N_{1}\right) \cdot \operatorname{Sp}\left(N_{2}\right) .
$$

Damit folgt für die Matrizen $\tilde{A}$ und $F$ aus 3.35 mit der Relation aus B.15

$$
\operatorname{Sp}(\underbrace{(\tilde{A} \otimes F) \cdot \ldots \cdot(\tilde{A} \otimes F)}_{\text {k-mal }})=\operatorname{Sp}\left(\tilde{A}^{k} \otimes F^{k}\right)=\operatorname{Sp}\left(\tilde{A}^{k}\right) \operatorname{Sp}\left(F^{k}\right) .
$$

\section{B.5. Erläuterungen zu verwendeten Reihen}

\section{Einschub zu den Polygamma-Funktionen:}

Dieser Einschub zu den Polygamma-Funktionen folgt 27 .

Da wir zur Bestimmung der unten stehenden Reihen (B.22) und (B.25) auf PolygammaFunktionen stoßen werden, gehen wir kurz auf diese ein. Zuerst sei die Hurwitzsche Zeta-Funktion erwähnt, die definiert ist durch

$$
\zeta(c, q)=\sum_{n=0}^{\infty} \frac{1}{(n+q)^{c}},
$$

mit $c \in \mathbb{C}, \operatorname{Re} z>1$ und $q \neq 0,-1,-2, \ldots$ Die Polygamma-Funktion $m$-ter Ordnung ist definiert als

$$
\Psi^{(m)}(q)=\frac{d^{m+1}}{d q^{m+1}} \log \Gamma(q)=\frac{d^{m}}{d q^{m}} \Psi^{(0)}(q), \quad m \in \mathbb{N},
$$

und aus der Entwicklung der Laurent-Reihe von $\zeta(c, q)$ an der Polstelle $c=1$ findet man für diese

$$
\Psi^{(m)}(q)=(-1)^{m+1} m ! \zeta(m+1, q) .
$$

Schließlich können wir mit Hilfe von (B.20) unmittelbar die Reihendarstellungen der Polygamma-Funktionen nullter und erster Ordnung, auch Diagamma- bzw. TrigammaFunktion genannt, angeben:

$$
\Psi^{(0)}(q)=-\zeta(1, q)=-\sum_{n=0}^{\infty} \frac{1}{(n+q)} \quad \Psi^{(1)}(q)=\zeta(2, q)=\sum_{n=0}^{\infty} \frac{1}{(n+q)^{2}} .
$$

\section{Zur Berechnung der hier gebrauchten unendlichen Reihen}

Bei der Bestimmung der Force-Extension-Relation treten zwei Typen von unendlichen Reihen auf, deren Wert wir benötigen. Mit den in (3.22) eingeführten Größen gilt im Allgemeinen mit $c_{a}:=\frac{\sqrt{z}}{a \pi}$ und für $a, b \neq 0$ :

$$
\begin{aligned}
& \frac{4 g}{L} \sum_{n=0}^{\infty} \frac{1}{\left(\frac{\pi(a n+b)}{L}\right)^{2} \cdot\left(\kappa\left(\frac{\pi(a n+b)}{L}\right)^{2}+f\right)}=\frac{4 \tilde{g} \tilde{T}}{z} \sum_{n=0}^{\infty}\left(\frac{1}{\pi^{2}(a n+b)^{2}}-\frac{1}{z+\pi^{2}(a n+b)^{2}}\right) \\
& =\frac{4 \tilde{g} \tilde{T}}{z}\left[\frac{1}{(a \pi)^{2}} \Psi^{(1)}\left(\frac{b}{a}\right)+\frac{i}{2 \pi a \sqrt{z}}\left(\Psi^{(0)}\left(\frac{b}{a}+i c_{a}\right)-\Psi^{(0)}\left(\frac{b}{a}-i c_{a}\right)\right)\right],
\end{aligned}
$$


wobei berücksichtigt wurde, dass

$$
\frac{1}{\pi^{2}(a n+b)^{2} \cdot\left[\pi^{2}(a n+b)^{2}+z\right]}=\left(\frac{1}{z}\right) \cdot\left[\frac{1}{\pi^{2}(a n+b)^{2}}-\frac{1}{z+\pi^{2}(a n+b)^{2}}\right]
$$

sowie

$$
-\sum_{n=0}^{\infty} \frac{1}{z+\pi^{2}(a n+b)^{2}}=\left(\frac{i}{2 \pi a \sqrt{z}}\right) \cdot \underbrace{\sum_{n=0}^{\infty}\left(\frac{1}{\left(n+\frac{b}{a}-i c_{a}\right)}-\frac{1}{\left(n+\frac{b}{a}+i c_{a}\right)}\right)}_{\Psi^{(0)}\left(\frac{b}{a}+i c_{a}\right)-\Psi^{(0)}\left(\frac{b}{a}-i c_{a}\right)}
$$

gilt. Außerdem erscheint in unserer Berechnung eine weitere konvergente Reihe, für die mit $a, b \neq 0$ gilt:

$$
\begin{aligned}
& \frac{g T}{L^{2}} \sum_{n=0}^{\infty} \frac{1}{\left(\frac{\pi(a n+b)}{L}\right)^{2} \cdot\left(\kappa\left(\frac{\pi(a n+b)}{L}\right)^{2}+f\right)^{2}}=\tilde{g} \tilde{T}^{2} \sum_{n=0}^{\infty} \frac{1}{\pi^{2}(a n+b)^{2} \cdot\left[\pi^{2}(a n+b)^{2}+z\right]^{2}} \\
& =\frac{\tilde{g} \tilde{T}^{2}}{z^{2}} \sum_{n=0}^{\infty}\left(\frac{1}{\pi^{2}(a n+b)^{2}}-\frac{1}{z+\pi^{2}(a n+b)^{2}}-\frac{1}{\left(\sqrt{z}+\pi^{2}(a n+b)^{2} z^{-1 / 2}\right)^{2}}\right) \\
& =\frac{\tilde{g} \tilde{T}^{2}}{z^{2}}\left[\frac{1}{(a \pi)^{2}} \Psi^{(1)}\left(\frac{b}{a}\right)+\frac{3 i}{4 \pi a \sqrt{z}}\left(\Psi^{(0)}\left(\frac{b}{a}+i c_{a}\right)-\Psi^{(0)}\left(\frac{b}{a}-i c_{a}\right)\right)\right. \\
& \left.+\frac{1}{4(\pi a)^{2}}\left(\Psi^{(1)}\left(\frac{b}{a}-i c_{a}\right)+\Psi^{(1)}\left(\frac{b}{a}+i c_{a}\right)\right)\right] .
\end{aligned}
$$

Hierbei erkennt man, dass die ersten beiden Terme der Partialbruchzerlegung von (B.25) ebenso in (B.22) auftauchen. 



\section{Literaturverzeichnis}

[1] A. Ashkin, J. M. Dziedzic, J. E. Buorkholm And S. Chu, Observation of a single-beam gradient force optical trap for dielectric particles, Optics Letters 11, 288-290 (1986)

[2] G. E. Astrakharchik, Quantum Monte Carlo Study of Ultracold Gases, arXiv:1412.4529, Dissertation, University of Trento, Italy (2004)

[3] K. R. Ayscough, In vivo functions of actin-binding proteins, Current Opinion in Cell Biology 10, 102-111 (1998)

[4] J. R. Bartles, Parallel Actin Bundles and Their Multiple Actin-bundling Proteins, Current Opinion in Cell Biology 12, 72-78 (2000)

[5] J. Baschnagel, J. P. Wittmer and H. Meyer, Monte Carlo Simulation of Polymers: Coarse-Grained Models in Computational Soft Matter: From Synthetic Polymers to Proteins, edited by N. Attig et al., John von Neumann Institute for Computing, Jülich, NIC Series 23, 83-140 (2004)

[6] A. R. Bausch And K. Kroy, A bottom-up approach to cell mechanics, Nature Physics 2, 231-238 (2006)

[7] E. Behrmann, M. Mueller, P. A. Penczek, H. G. Mannherz, D. J. Manstein AND S. RAUNSER, Structure of the rigor actin-tropomyosin-myosin complex, Cell 150, 327-338 (2012), PDB-ID: 4a7n

[8] P. Benetatos And E. Frey, Depinning of semiflexible polymers, Physical Review E 67, 051108 (2003)

[9] P. Benetatos And E. Frey, Linear response of a grafted semiflexible polymer to a uniform force field, Physical Review E 70, 051806 (2004)

[10] P. Benetatos and A. Zippelius, Anisotropic Random Networks of Semiflexible Polymers, Physical Review Letters 99, 198301 (2007)

[11] P. Benetatos And E. M. Terentuev, Stretching weakly bending filaments with spotaneous curvature in two dimensions, Physical Review E 81, 031802 (2010)

[12] P. Benetatos And E. M. Terentuev, Stretching semiflexible filaments with quenched disorder, Physical Review E 82, 050802(R) (2010)

[13] P. Benetatos, S. Ulrich and A. Zippelius, Force-Extension relation of crosslinked anisotropic polymer networks, New Journal of Physics 14, 115011, (2012)

[14] P. Benetatos, E. M. Terentuev And A. Zippelius, Bundling in brushes of directed and semiflexible polymers, Physical Review E 88, 042601 (2013) 
[15] P. Benetatos, A. V. D. Heydt and A. Zippelius, Tension-induced binding of semiflexible biopolymers, New Journal of Physics 16, 113037 (2014)

[16] D. S. Bernstein, Matrix Mathematics: Theory, Facts, and Formulas, Second Edition, Princeton University Press (2009)

[17] K. Binder and D. W. Heermann, Monte Carlo Simulation in Statistical Physics - An Introduction, 5th Edition, Springer-Verlag Berlin Heidelberg (2010)

[18] M. Burger, Numerik partieller Differentialgleichungen, Vorlesungsskript Universität Münster (2007)

[19] C. Bustamante, J. F. Marko, E. D. Siggia and S. Smith, Entropic Elasticity of $\lambda$-Phage DNA, Science 265, 1599-1600 (1994)

[20] C. Bustamante, S. B. Smith, J. Liphardt and D. Smith, Single-molecule studies of DNA mechanics, Current Opinion in Structural Biology 10, 279-285 (2000)

[21] G. M. Cooper and R. E. Hausman, The Cell: A Molecular Approach, 6th Edition, Sinauer Associates, Inc. Publishers, Sunderland, Massachusetts U.S.A. (2013)

[22] H. Dehling und B. Haupt, Einführung in die Wahrscheinlichkeitstheorie und Statistik, 2. Auflage, Springer-Verlag Berlin Heidelberg New York, (2004)

[23] M. Doi and S. F. Edwards, The Theory of Polymer Dynamics, Oxford University Press, New York (1986)

[24] D. E. Dupuis, W. H. Guilford, J. Wu and D. M. Warshaw, Actin filament mechanics in the laser trap, Journal of Muscle Research and Cell Motility 18, 17-30 (1997)

[25] S. F. EDwards, The statistical mechanics of polymers with excluded volume, Proceedings of the Physical Society 85, 613 (1965)

[26] L. Eichinger, B. Köppel, A. A. Noegel, M. Schleicher, M. Schliwa, K. WeiJer, W. Witke AND P. A. JANMEY, Mechanical pertubation elicits a phenotypic difference between Dictyostelium wild-type cells and cytoskeletal mutants, Biophysical Journal 70, 1054-1060 (1996)

[27] O. Espinosa And V. H. Moll, A Generalized Polygamma Function, Integral Transforms and Special Functions 15, 101-115 (2004)

[28] R. Everaers, R. Bundschun And K. Kremer, Fluctuations and Stiffness of Double-Stranded Polymers: Railway-Track Model, Europhysics Letters 29, 263268 (1995)

[29] H. W. Eves, Elementary Matrix Theory, Dover Publications, Inc. New York (1980)

[30] G. Fischer, Lineare Algebra, 15. Auflage, Friedr. Vieweg \& Sohn Verlag / GWV Fachverlage GmbH, Wiesbaden (2005)

[31] D. A. Fletcher And R. D. Mullins, Cell mechanics and the cytoskeleton, Nature 463, 485-492 (2010) 
[32] P. J. Flony, Principles of Polymer Chemistry, Cornell University Press, Ithaca, New York (1953)

[33] J. S. Frame, A Simple Recursion Formula for Inverting a Matrix, Bulletin of the American Mathematical Society 55, 1045 (1949)

[34] D. Frenkel And B. Smit, Understanding Molecular Simulation, Second Edition, Academic Press San Diego (2002)

[35] E. Frey, K. Kroy, J. Wilhelm and E. Sackmann, Statistical Mechanics of Semiflexible Polymers: Theory and Experiment, arXiv:cond-mat/9707021v2 (1998)

[36] F. R. Gantmacher, Matrizentheorie, Springer-Verlag Berlin (1986)

[37] M. L. Gardel, J. H. Shin, F. C. MacKintosh, L. Mahadevan, P. MatsuDAIRA AND D. A. Weitz, Elastic Behavior os Cross-Linked and Bundled Actin Networks, Science 304, 1301-1305 (2004)

[38] P.-G. DE Gennes, Some conformation problems for long macromolecules, Reports on Progress in Physics 32, 187 (1969)

[39] P.-G. DE Gennes, Scaling concepts in polymer physics, Cornell University Press, Ithaca, New York (1979)

[40] A. Gholami, J. Wilhelm and E. Frey, Entropic forces generated by grafted semiflexible polymers, Physical Review E 74, 041803 (2006)

[41] F. Gittes, B. Mickey, J. Nettleton and J. Howard, Flexural Rigidity of Microtubules and Actin Filaments Measured from Thermal Fluctuations in Shape, The Journal of Cell Biology 120, 923-934 (1993)

[42] G. H. Golub and C. F. Van Loan, Matrix Computations, 4th Edition, The Johns Hopkins University Press Baltimore, Maryland (2013)

[43] D. Greenspan, Methods of Matrix Inversion, The American Mathematical Monthly 62, 303-318 (1955)

[44] W. Greiner und J. Reinhardt, Theoretische Physik, Band 7A: Feldquantisierung, Verlag Harri Deutsch, Thun und Frankfurt am Main (1993)

[45] G. GRinstein And A. Luther, Application of the renormalization group to phase transitions in disordered systems, Physical Review B 13, 1329-1343 (1976)

[46] A. Y. Grosberg And A. R. Khokhlov, Statistical Physics of Macromolecules, AIP Press Woodbury, New York (1994)

[47] P. Gutjahr, R. Lipowsky and J. Kierfeld, Persistence length of semiflexible polymers and bending rigidity renormalization, Europhysics Letters 76, 994-1000 (2006)

[48] J.-P. Hansen And I. R. McDonald, Theory of Simple Liquids, Second Edition, Academic Press Limited, London (1986)

[49] D. A. Head, A. J. Levine and F. C. MacKintosh, Deformation of CrossLinked Semiflexible Polymer Networks, Physical Review Letters 91, 108102 (2003) 
[50] D. W. Heermann, Theoretical Biophysics, Vorlesungsskript Universität Heidelberg (2006)

[51] A. V. D. Heydt, D. Wilkin, P. Benetatos and A. Zippelius, Elasticity of cross-linked semiflexible biopolymers under tension, Physical Review E 88, 032701 (2013)

[52] C. Heussinger, M. Bathe and E. Frey, Statistical Mechanics of Semiflexible Bundles of Wormlike Polymer Chains, Physical Review Letters 99, 048101 (2007)

[53] K. C. Holmes, D. Popp, W. Gebhard, W. Kabsch, Atomic model of the actin filament, Nature 347, 44-49 (1990)

[54] R. A. Horn and C. R. Johnson, Topics in Matrix Analysis, Cambridge University Press (1991)

[55] R. A. Horn and C. R. Johnson, Matrix Analysis, Second Edition, Cambridge University Press (2013)

[56] H.-P. Hsu, W. Paul and K. Binder, Breakdown of the Kratky-Porod Wormlike Chain model for semiflexible polymers in two dimensions, Europhysics Letters, 95, $68004(2011)$

[57] F. Huber, J. Schnauss, S. Rönicke, P. Rauch, K. Müller, C. Fütterer AND J. KÄs, Emergent complexity of the cytoskeleton: from single filaments to tissue, Advances in Physics 62, 1-112 (2013)

[58] B. A. H. Huisman, P. G. Bolhuis and A. Fasolino, Phase Transition to Bundles of Flexible Supramolecular Polymers, Physical Review Letters 100, $188301(2008)$

[59] W. Humphrey, A. Dalke And K. Schulten, VMD: Visual Molecular Dynamics, Journal of Molecular Graphics 14, 33-38 (1996), http://www.ks.uiuc.edu/Research/vmd/

[60] Y. ImRY AND M. WorTis, Influence of quenched impurities on first-order phase transitions, Physical Review B 19, 3580-3585 (1979)

[61] P. A. Janmey, S. Hvidt, J. Lamb and T. P. Stossel, Resemblance of actinbinding protein/actin gels to covalently crosslinked networks, Nature $\mathbf{3 4 5}, 89-92$ (1990)

[62] P. A. JAnmeY, Mechanical properties of cytoskeletal polymers, Current Opinion In Cell Biology 3, 4-11 (1991)

[63] J. E. Jones, On the Determination of Molecular Fields. I. From the Variation of the Viscosity of a Gas with Temperature, Proceedings of the Royal Society of London A: Mathematical, Physical and Engineering Sciences 106, 441-462 (1924)

[64] T. Kawakatsu, Statistical Physics of Polymers: An Introduction, SpringerVerlag Berlin Heidelberg (2004)

[65] J. KieRFELD AND R. Lipowsky, Unbundling and desorption of semiflexible polymers, Europhysics Letters 62, 285-291 (2003) 
[66] J. Kierfeld, T. KÜHne And R. Lipowsky, Discontinuous Unbinding Transitions of Filament Bundles, Physical Review Letters 95, 038102 (2005)

[67] J. Kierfeld, P. Gutjahr, T. Kühne, P. Kraikivski and R. Lipowsky, Buckling, Bundling, and Pattern Formation: From Semi-Flexible Polymers to Assemblies of Interacting Filaments, Journal of Computational and Theoretical Nanoscience 3, 898-911 (2006)

[68] J. Kierfeld, Semiflexible Polymere und Filamente: Vom einzelnen Polymer zur biologischen Struktur, Forschungsbericht 2007 - Max-Planck-Institut für Kolloidund Grenzflächenforschung (2007)

[69] H. Kojima, A. Ishijima And T. Yanagida, Direct measurement of stiffness of single actin filaments with and without tropomyosin by in vitro nanomanipulation, Proceedings of the National Academy of Science of the United States of America 91, 12962-12966 (1994)

[70] S. Köster, H. Stark, T. Pfohl and J. Kierfeld, Fluctuations of single confined actin filaments, Biophysical Review Letters 2, 155-166 (2007)

[71] S. Köster and T. Pfohl, An In Vitro Model System for Cytoskeletal Confinement, Cell Motility and the Cytoskeleton 66, 771-776 (2009)

[72] O. Kratky and G. Porod, Röntgenuntersuchung gelöster Fadenmoleküle, Recueil des Travaux Chimiques des Pays-Bas 68, 1106-1122 (1949)

[73] U. KRENGEL, Einführung in die Wahrscheinlichkeitstheorie und Statistik, 8. erw. Auflage, Friedr. Vieweg \& Sohn Verlag / GWV Fachverlage GmbH, Wiesbaden (2005)

[74] K. Kroy and E. Frey, Force-Extension Relation and Plateau Modulus for Wormlike Chains, Physical Review Letters 77, 306 (1996)

[75] K. Kroy, Viskoelastizität von Lösungen halbsteifer Polymere, Hieronymus Verlag, Dissertation, TU München, (1998)

[76] W. KüHnel, Differentialgeometrie, Kurven-Flächen-Mannigfaltigkeiten, 3. überarb. Auflage, Friedr. Vieweg \& Sohn Verlag / GWV Fachverlage GmbH, Wiesbaden (2008)

[77] P.-Y. LAi AND K. Binder, Structure and dynamics of grafted polymer layers: A Monte Carlo Simulation, The Journal of Chemical Physics 95, 9288 (1991)

[78] P.-Y. LAI, Computer simulations of end-grafted polymer chains, Physica A 205, 191-197 (1994)

[79] L. D. Landau and E. M. Lifshitz, Theory of Elasticity, Third Edition, Pergamon Press, Oxford/New York (1986)

[80] O. Lieleg And A. R. Bausch, Cross-Linker Unbinding and Self-Similarity in Bundled Cytoskeletal Networks, Physical Review Letters 99, 158105 (2007)

[81] O. Lieleg, M. M. A. E. Claessens, C. Heussinger, E. Frey and A. R. Bausch, Mechanics of Bundled Semiflexible Polymer Networks, Physical Review Letters 99, 088102 (2007) 
[82] O. Lieleg, K. M. Schmoller, M. M. A. E. Claessens and A. R. Bausch, Cytoskeletal Polymer Networks: Viscoelastic Properties are Determined by the Microscopic Interaction Potential of Cross-Links, Biophysical Journal 96, 4725$4732(2009)$

[83] X. Liu And G. H. Pollack, Mechanics of F-Actin Characterized with Microfabricated Cantilevers, Biophysical Journal 83, 2705-2715 (2002)

[84] T. B. Liverpool, R. Golestanian and K. Kremer, Statistical Mechanics of Double-Stranded Semiflexible Polymers, Physical Review Letters 80, 405 (1998)

[85] D. M. Loveless, N. I. Abu-Lail, M. Kaholek, S. Zauscher and S. L. Craig, Reversibly Cross-Linked Surface-Grafted Polymer Brushes, Angewandte Chemie International Edition 45, 7812-7814 (2006)

[86] F. C. Mackintosh, J. Käs and P. A. Janmey, Elasticity of Semiflexible Biopolymer Networks, Physical Review Letters 75, 4425 (1995)

[87] J. F. Marko AND E. D. Siggia, Streching DNA, Macromolecules 28, 8759-8770 (1995)

[88] N. Metropolis, A. W. Rosenbluth, M. N. Rosenbluth, A. H. Teller AND E. Teller, Equation of State Calculations by Fast Computing Machines, The Journal of Chemical Physics 21, 1087 (1953)

[89] S. T. Milner, Polymer Brushes, Science 251, 205 (1991)

[90] G. Morandi, F. Napoli and E. Ercolessi, Statistical Mechanics: An Intermediate Course, Second Edition, World Scientific Publishing, Singapore (2001)

[91] M. E. J. Newman and G. T. Barkema, Monte-Carlo Methods in Statistical Physics, Clarendon Press Oxford (1999)

[92] J.-F. Nolting, W. MöBius And S. Köster, Mechanics of Individual Keratin Bundles in Living Cells, Biophysical Journal 107, 2693-2699 (2014)

[93] A. Ott, M. Magnasco, A. Simon And A. Libchaber, Measurement of the persistence length of polymerized actin using fluorescence microscopy, Physical Review E 48, R1642 (1993)

[94] S. K. Pattanayek, T. T. Pham and G. G. Pereira, Morphological structures formed by grafted polymers in poor solvents, The Journal of Chemical Physics 122, 214908 (2005)

[95] S. Persson, A. Sampathkumar und M. Bringmann, Zwei Skelette treffen sich: die pflanzliche Zellwand und das Zytoskelett, Forschungsbericht 2011 - MaxPlanck-Institut für molekulare Pflanzenphysiologie (2011)

[96] T. T. Pham, S. K. Pattanayek and G. G. Pereira, Semiflexible grafted polymers in poor solvents: Toroidal, archway, and tower micelles, The Journal of Chemical Physics 123, 034904 (2005)

[97] A. Prasad, Y. Hori and J. Kondev, Elasticity of semiflexible polymers in two dimensions, Physical Review E 72, 041918 (2005) 
[98] P. K. Purohit, M. E. Arsenault, Y. Goldman and H. H. Bau, The mechanics of short rod-like molecules in tension, International Journal of NonLinear Mechanics 43, 1056-1063 (2008)

[99] M. Rubinstein and R. H. Colby, Polymer Physics, Oxford University Press Oxford (2003)

[100] N. Saito, K. Takahashi and Y. Yunoki, The Statistical Mechanical Theory of Stiff Chains, Journal of the Physical Society of Japan 22, 219-226 (1967)

[101] M. Schwabl, Statistische Mechanik, 3. Auflage, Springer-Verlag Berlin Heidelberg (2006)

[102] H. R. Schwarz und N. KöcKLer, Numerische Mathematik, 8. Auflage, Vieweg + Teubner Verlag | Springer Fachmedien Wiesbaden GmbH, Wiesbaden (2011)

[103] J. Sherman and W. J. Morrison, Adjustment of an Inverse Matrix Corresponding to Changes in the Elements of a Given Column or a Given Row of the Original Matrix (abstract), The Annals of Mathematical Statistics 20, 621 (1949)

[104] J. Sherman And W. J. Morrison, Adjustment of an Inverse Matrix Corresponding to a Change in One Element of a Given Matrix, The Annals of Mathematical Statistics 21, 124-127 (1950)

[105] S. B. Smith, Y. Cui And C. Bustamante, Overstretching B-DNA: The Elastic Response of Individual Double-Stranded and Single-Stranded DNA Molecules, Science 271, 795-799 (1996)

[106] W.-H. SteEB, Kronecker Product of Matrices and Applications, BIWissenschaftsverlag Mannheim (1991)

[107] G. Strang, Introduction to Linear Algebra, Fourth Edition, WellesleyCambridge Press (2009)

[108] G. Strobl, The Physics of Polymers: Concepts for Understanding Their Structures and Behaviour, Third Edition, Springer-Verlag Berlin Heidelberg (2007)

[109] S. Ulrich, A. Zippelius and P. Benetatos, Random networks of cross-linked directed polymers, Physical Review E 81, 021802 (2010)

[110] C. F. VAn LoAn, The ubiquitous Kronecker product, Journal of Computational and Applied Mathematics 123, 85-100 (2000)

[111] R. L. C. Vink, Monte-Carlo Methods in Statistical Physics, Vorlesungsnotizen Universität Göttingen, (2011)

[112] R. L. C. Vink and C. Heussinger, Cross-linked biopolymer bundles: Crosslink reversibility leads to cooperative binding/unbinding phenomena, The Journal of Chemical Physics 136, 035102 (2012)

[113] M. D. Wang, H. Yin, R. Landick, J. Gelles and S. M. Block, Stretching DNA with Optical Tweezers, Biophysical Journal 72, 1335-1346 (1997)

[114] M. Warner and E. M. Terentjev, Liquid Crystal Elastomers, Clarendon Press, Oxford (2003) 
[115] B. Weinhausen, Unveröffentlichte Aufnahmen, Forschungsgruppe „Nanoscale Imaging of Cellular Dynamics", Georg-August-Universität Göttingen

[116] L. Wenning, M. Müller And K. Binder, How does the pattern of GraftingPoints influence the structure of one-component and mixed Polymer Brushes?, Europhysics Letters 71, 639-645 (2005)

[117] J. Wilhelm and E. Frey, Elasticity of Stiff Polymer Networks, Physical Review Letters 91, 108103 (2003)

[118] J. Xu, W. H. Schwarz, J. A. Käs, T. P. Stossel, P. A. Janmey and T. D. Pollard, Mechanical Properties of Actin Filament Networks Depend on Preparation, Polymerization Conditions, and Storage of Actin Monomers, Biophysical Journal 74, 2731-2740 (1998)

[119] B. Zhao And W. J. Brittain, Polymer brushes: surface-immobilized macromolecules, Progress in Polymer Science 25, 677-710 (2000) 


\section{Danksagung}

Die Promotionszeit in Göttingen wird mir mit Sicherheit stets in positiver Erinnerung bleiben. An dieser Stelle möchte ich gerne denjenigen Menschen meinen Dank aussprechen, die mich bei der Erstellung dieser Arbeit in unterschiedlicher Weise unterstützt und begleitet haben.

- Zuallererst danke ich Frau Prof. Dr. Annette Zippelius für die Aufnahme in ihre Forschungsgruppe und für die Möglichkeit, an einem interessanten und spannenden Aufgabengebiet arbeiten zu können. Bedanken möchte ich mich auch für die gute Betreuung und die zahlreichen, wertvollen Ratschläge aus den Besprechungen der letzten Jahre.

- Ebenfalls bedanken möchte ich mich bei Herrn Prof. Dr. Reiner Kree für die Bereitschaft, das Korreferat zu übernehmen.

- Ein weiterer Dank gilt Herrn Dr. Richard Vink, der mich im Zusammenhang mit dem numerischen Teil betreut hat. Seine engagierte Unterstützung mit vielen wichtigen Anregungen während der Betreuungsphase weiß ich sehr zu schätzen.

- Herrn Prof. Panayotis Benetatos möchte ich meinen Dank für die gute und angenehme Zusammenarbeit äußern.

- Ein weiteres, spezielles Dankeschön möchte ich Frau Dr. Alice von der Heydt aussprechen. Vielen Dank für die stets gute Zusammenarbeit, die ertragreichen Diskussionen und hilfreichen Anmerkungen.

- Selbstverständlich bedanke ich mich herzlich bei allen Institutsmitgliedern für die schöne gemeinsame Zeit und die angenehme Atmosphäre.

- Zu guter Letzt geht ein herzlicher Dank an meine Familie. 



\section{Lebenslauf}

\section{Persönliche Daten}

Name:

Geburtstag und -ort:

Adresse:

Nationalität:

\section{Schulausbildung}

1990-1999

Juni 1999

Wehrersatzdienst

1999-2000

\section{Studium}

2001-2007

April 2003

April 2007

2007-2011

Mai 2011

Juli 2011
Daniel Wilkin

28. März 1980 in Speyer

Rosdorfer Weg 30, 37073 Göttingen

deutsch

Hans-Purrmann-Gymnasium Speyer

Abitur

Zivildienst Diakonissenkrankenhaus Speyer

Studium der Physik an der Ruprecht-Karls-Universität Heidelberg, Diplomarbeit:,Aufbau eines Kollimationssystems und Entwicklung eines Scanners für das Spektrometer PERKEO III" bei Prof. Dr. Hartmut Abele, Physikalisches Institut

Diplom-Vorprüfung Physik

Diplom Physik, Abschluss

Studium der Werkstoffwissenschaft an der Friedrich-SchillerUniversität Jena, Diplomarbeit:,Vielteilchenwechselwirkungen von Elektronen in einem Hubbard-Cluster" bei Prof. Dr.

Friedhelm Bechstedt, Institut für Festkörpertheorie und -optik

Diplom Werkstoffwissenschaft, Abschluss

Beginn des Promotionsstudiums an der Georg-August-Universität Göttingen bei Prof. Dr. Annette Zippelius, Institut für Theoretische Physik

\section{Publikation}

- A. v. D. Heydt, D. Wilkin, P. Benetatos and A. Zippelius. Elasticity of cross-linked semiflexible biopolymers under tension. Phys. Rev. E 88, 032701 (2013) 